\title{
Gut bacterial fermentation of food by-products
}

Citation for published version (APA):

Bussolo de Souza, C. (2019). Gut bacterial fermentation of food by-products. [Doctoral Thesis, Maastricht University]. Gildeprint Drukkerijen. https://doi.org/10.26481/dis.20190213cb

Document status and date:

Published: 01/01/2019

DOI:

10.26481/dis.20190213cb

Document Version:

Publisher's PDF, also known as Version of record

\section{Please check the document version of this publication:}

- A submitted manuscript is the version of the article upon submission and before peer-review. There can be important differences between the submitted version and the official published version of record.

People interested in the research are advised to contact the author for the final version of the publication, or visit the DOI to the publisher's website.

- The final author version and the galley proof are versions of the publication after peer review.

- The final published version features the final layout of the paper including the volume, issue and page numbers.

Link to publication

\footnotetext{
General rights rights.

- You may freely distribute the URL identifying the publication in the public portal. please follow below link for the End User Agreement:

www.umlib.nl/taverne-license

Take down policy

If you believe that this document breaches copyright please contact us at:

repository@maastrichtuniversity.nl

providing details and we will investigate your claim.
}

Copyright and moral rights for the publications made accessible in the public portal are retained by the authors and/or other copyright owners and it is a condition of accessing publications that users recognise and abide by the legal requirements associated with these

- Users may download and print one copy of any publication from the public portal for the purpose of private study or research.

- You may not further distribute the material or use it for any profit-making activity or commercial gain

If the publication is distributed under the terms of Article $25 \mathrm{fa}$ of the Dutch Copyright Act, indicated by the "Taverne" license above, 
Gut bacterial fermentation of food by-products

Carlota Bussolo de Souza 
The research described in this $\mathrm{PhD}$ thesis was part of the program entitled "Science without borders" launched in 2011 by the Brazilian government - CNPq (National Council for Scientific and Technological Development), grant number 246027/2012-6. Legal representative in Brazil: Prof. Dr. Susana Saad (USP).

Cover-design: Carlota Bussolo de Souza and GildePrint Layout: Carlota Bussolo de Souza

Painting: Zélia Bussolo

Printed by: GildePrint, Enschede, the Netherlands

ISBN: 978-94-6323-496-2

Thesis Maastricht University

All rights reserved. No part of this thesis may be reproduced, distributed, stored in a retrieval system, or transmitted in any form or by any means without prior permission of the author. 


\title{
Gut bacterial fermentation of food by-products
}

\author{
DISSERTATION \\ to obtain the degree of Doctor at \\ Maastricht University on the authority of the Rector Magnificus \\ Prof. Dr. Rianne M. Letschert \\ in accordance with the decision of the Board of Deans, \\ to be defended in public \\ on Wednesday $13^{\text {th }}$ of February 2019 at 10.00 hours
}

by

Carlota Bussolo de Souza 


\section{Supervisors:}

Prof. Koen Venema

Prof. Lubbert Dijkhuizen, University of Groningen

\section{Co-supervisor:}

Prof. Dr. Daisy Jonkers

\section{Assessment Committee:}

Prof. Dr. P.H.M. Savelkoul, Chairman

Prof. Dr. E.E. Blaak

Prof. Dr. S.G. Sayago-Ayerdi, Mexico

Prof. Dr. K. Verbeke, KU Leuven 


\section{Content}

Chapter 1

Chapter 2

Chapter 3

Chapter 4

Chapter 5

Chapter 6

Chapter 7

Chapter 8

Summary

Samenvatting

Valorization

Acknowledgments

About the author
General introduction

7

Characterization and in

37

vitro digestibility of by-products from

Brazilian food industry: Cassava

bagasse, orange bagasse and passion

fruit peel

Prebiotic effects of cassava bagasse in

61

TNO's in vitro model of the colon in lean versus obese microbiota

Lean and obese microbiota: differences in in vitro fermentation of by-products from Brazilian food industry

Degradation of fibres from fruit by127 products allows selective modulation of the gut bacteria in an in vitro model of the proximal colon

The gut microbiota from lean and obese 161 subjects contribute differently to the fermentation of arabinogalactan and inulin

Potential of pectins to beneficially 191 modulate the gut microbiota depends on their structural properties

General discussion

221

239

243

249

257

261 



\section{Chapter 1}

General introduction

Partly published as:

Bussolo de Souza, C., Venema, K. (2017). Microbiota intestinal em indivíduos eutróficos e obesos. In Microbioma, disbiose, probióticos e bacterioterapia. (pp. 164-177). São Paulo, SP: Manole (in Portuguese). 


\section{Background - reasons for this research}

The gut microbiota has been shown to be important in health and disease. The main focus of the research described in this thesis was to investigate the potential prebiotic effects on the gut microbiota of food by-products from the Brazilian processing industry, with the aim to create a more healthy gut microbiota, e.g. in obesity. To accomplish this, waste-streams of cassava, orange and passion fruit were initially chosen for investigation. Differences in fermentation were assessed when using a microbiota originating from lean or obese individuals. During the research, in addition to the Brazilian food byproducts the fermentable fibres arabinogalactan and inulin were used. The effects of different types of pectins extracted from diverse food by-products were also evaluated. The approach used was in vitro fermentation in the validated TIM-2 model. Gut microbiota compositional changes, metabolite production and characterization of the intermediate degradation products in the chyme were evaluated upon addition of the different substrates. The knowledge gained in this research provides the basis for further research aiming at the valorisation of these by-products and the results showed that the by-products could be used as an alternative tool to improve intestinal health and consequently offer an additional approach to tackle obesity. The background and aims are further highlighted in the following paragraph, which is the introductory chapter to the thesis.

\section{Human gastrointestinal tract and gut microbiota - the basics}

The human gastrointestinal tract is a 7-10 metres long hollow tube that begins at the mouth and ends at the anus. It is composed of specific parts: mouth, oesophagus, stomach, small intestine, large intestine, rectum and anus $(1,2)$. The small intestine is 2.8-8.5 meters long and it is divided into 3 distinct parts: duodenum (20-30 cm long), jejunum (upper two-fifths of the small intestine) and ileum (lower three-fifths of the small intestine) (2). The large intestine is around 1.5 meters long and includes the cecum, proximal colon, transverse colon, distal colon, rectum and anus (1). The absorptive surface of the intestine is amplified by specific structures (folds in the intestinal wall, villi and microvilli), making a total area of around $200 \mathrm{~m}^{2}(2)$. Together with assistant organs - salivary glands, liver, gallbladder and pancreas - the process of digestion and absorption of nutrients is accomplished.

For a long time the large intestine was viewed as a storage place for undigested food components (3). Nevertheless, nowadays it is called the "forgotten organ" (4) thanks to the metabolic potential of the complex assemblage of different microorganisms that reside there - the gut microbiota. This metabolic potential 
is considered equal to that of the liver (3). The gut microbiota is a complex community composed of bacteria, archaea and eukarya, living in our gastrointestinal tract (mostly in the colon) in a mutual beneficial relationship $(5,6)$. It is estimated that this community encompasses $10^{10}$ to $10^{11}$ bacterial cells per gram of faecal material, and the microbiome (genes from microbiota) to have around 9.9 million genes - 100 times more than the human genome $(6,7)$. It corresponds to around $50 \%$ of the wet weight of faecal biomass in humans, which accounts for about 0.5-1.5 kilograms of bacteria in our gut (8). Bacteria dominate this ecosystem, and data from the Human Microbiome Project and MetaHit revealed that around $93 \%$ of the bacterial residents in our gut belong to the dominant phyla of Firmicutes, Bacteroidetes, Proteobacteria and Actinobacteria $(5,9)$.

The colonic microbiota ferments endogenous host-derived substrates such as mucus, pancreatic enzymes, and exfoliated epithelial cells, as well as dietary components that escape digestion in the upper gastrointestinal tract (10). Complex carbohydrates (resistant starch, non-starch polysaccharides) and proteins are the principal dietary products that reach the colon and serve as food for the gut microbiota (9). Two main types of colonic microbial fermentation can be distinguished: saccharolytic fermentation of carbohydrates and proteolytic fermentation of proteins $(10,11)$.

Most microorganisms prefer to ferment carbohydrates over proteins. Consequently, saccharolytic fermentation occurs predominantly in the proximal colon, while fermentation of proteins mainly takes place in the distal colon where fermentable carbohydrates are depleted and the bacteria switch to proteolytic fermentation $(9,12)$. Fermentation of proteins may lead to what are considered toxic metabolites, such as hydrogen sulfide, ammonia, branched chain fatty acids (BCFA) and phenolic and indolic compounds (10,11). As mentioned, fermentation of proteins becomes quantitatively more important distally, and the majority of colorectal cancers occur in the distal side of the colon. There, the concentration of proteolytic metabolites is higher, and contact of the intestinal epithelium with luminal contents is increased because of the more solid nature of luminal contents and also due to the slower transit through this segment of the bowel (3).

Nevertheless, bacterial fermentation also produces beneficial compounds, and this for instance occurs when the microbiota ferments carbohydrates. The end products of carbohydrate fermentation are the beneficial metabolites short chain fatty acids (SCFA), mainly acetate, propionate and butyrate, as well as lactate (9). SCFA are crucial for intestinal health, being used as energy substrate by colonocytes for instance, and have other important functions, among others 
anti-inflammatory activity, increasing the intestinal barrier, and playing a beneficial role in satiety and oxidative stress (13). Moreover, during carbohydrate fermentation, proteins are incorporated into microbial biomass, preventing their fermentation (11).

Consequently, the importance of the human large intestinal microbiota for nutrition, health and disease is becoming increasingly realized, and more studies are being performed with the aim to elucidate the mechanisms behind this complex interplay between the host and the gut microbiota.

\section{Dietary fibres - the main fuel of gut microbiota}

The composition and activity of the gut microbiota can be influenced by several external factors, with diet as one of the most important (14). In addition, among all nutrients present in our diet, fibres deserve a special attention when talking about intestinal health and gut microbiota. This is because the human gastrointestinal tract has a limited number of enzymes capable of digesting the vast range of different carbohydrates present in our diet (15). In contrast, the gut microbiome encodes biochemical pathways not evolved in humans (9). Therefore, the indigestible carbohydrates (i.e., dietary fibres) that reach the colon serve as energy source to the gut microbiota, which possesses most of the required enzymes to utilize those (15).

Currently, dietary fibre is defined as carbohydrate polymers with a degree of polymerization (DP) of three or more, which are neither digested nor absorbed in the human intestine (16), including:

- non-starch polysaccharides (NSP) from plants (e.g. fruits and tubers) whether intrinsic or extracted, chemically, physically and/or enzymatically modified or synthetic (DP $\geq 10$ );

- resistant (non-digestible) oligosaccharides (DP 3-9);

- resistant starch (RS) (DP $\geq 10)$.

Among the dietary fibres that seem to be effective in the context of gut health, prebiotics are now well described in the literature, and these can be used as a tool to modulate the gut microbiota. A prebiotic is defined as "a substrate that is selectively utilized by host microorganisms conferring a health benefit" (17). The genera Bifidobacterium and Lactobacillus are usually used as biomarkers for intestinal health and the prebiotic concept (18). The reason lies in the fact that these two genera do not contain any known pathogens and they use carbohydrates as preferred energy source, producing acetate and lactate as metabolites, which through cross-feeding can be converted in the other shortchain fatty acids (SCFA), e.g. butyrate which is known for its positive effects on the host (18). Other genera, such as Bacteroides, besides using carbohydrates for 
fermentation, also utilize proteins and amino acids as substrates for their energy metabolism. The metabolites from proteolytic fermentation are known by their harmful effects on the gut and are linked with colon cancer (19).

It is important to keep in mind that dietary fibre is a heterogeneous group of compounds with diverse chemical structures, and therefore different fibres will behave differently in our gastrointestinal tract (absorb more or less water, increase faecal bulking, etc.), they will be metabolized in different ways and be fermented (or not) by different bacterial groups. Consequently, the effects on host metabolism will be distinct for each type of fibre. Therefore, it is fundamental to characterize different fibres in order to predict their possible health effects, as well as to correlate their intake with the microbiota composition (increase/decrease in certain bacterial groups), type and amount of metabolites produced, fate of fermentation/degradation, and so on. Figure 1 demonstrates the classification of carbohydrates according to their chemical and physiological characteristics.

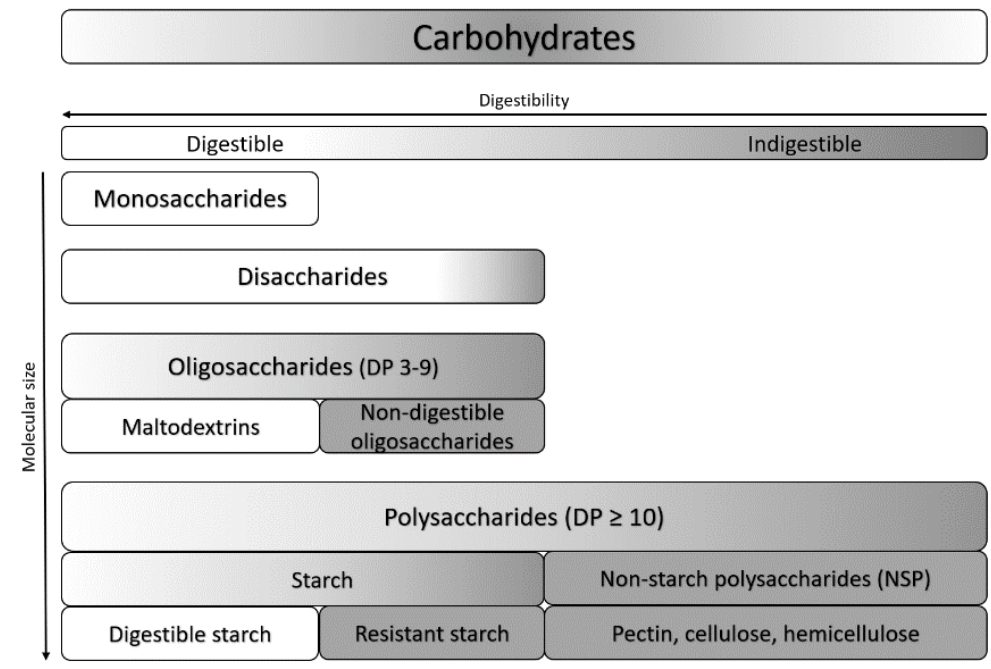

Figure 1: Carbohydrate classification according to their chemical and physiological characteristics. Adapted from (20).

Carbohydrates can be chemically classified according to their molecular size (degree of polymerization - DP) into three different groups (Figure 1):

- sugars (DP 1-2) that encompasses monosaccharides and disaccharides (e.g. glucose and sucrose);

- oligosaccharides (DP 3-9) that include maltodextrins and non-digestible oligosaccharides such as fructooligosaccharides (FOS);

- polysaccharides ( $\mathrm{DP} \geq 10$ ) which can be divided into starch and NSP (e.g. pectin and cellulose) (21). 
Moreover, carbohydrates can also be classified according to their digestibility in the small intestine - digestible and non-digestible (fibres) carbohydrates (Figure 1).

Since the focus of the thesis is on dietary fibres, a selection of the more relevant carbohydrates in this context is described below.

\section{Resistant starch}

Resistant starch is a polysaccharide composed of starch that consists of glucose monomers linked by $\alpha-(1,4)$ or $\alpha-(1,6)$ linkages $(22)$. It has been defined as "the sum of starch and products of starch degradation that are not absorbed in the small intestine of healthy individuals" (23). According to the determinant factor of resistance, RS is classified into four different types:

- RS type 1: physically inaccessible;

- RS type 2: present in granular form;

- RS type 3: retrograded;

- RS type 4: chemically modified (22).

A study comparing the effects of two types of RS (RS2 and RS4) on microbial composition revealed distinct compositional alterations and high individual differences in the dietary responsiveness (24). Despite the individual differences, RS4 increased the proportions of Bifidobacterium adolescentis and Parabacteroides distasonis, and RS2 stimulated the growth of Ruminococcus bromii and Eubacterium rectale (24). Another study using RS3 also demonstrated an increase in $R$. bromii, among others (25). Therefore, it is important to highlight that the difference in the type of RS should be taken into account, since it has been demonstrated that these differences produce diverse effects on health and gut microbiota.

\section{Cellulose}

Cellulose is a linear chain of glucose units connected by $\beta-(1,4)$ linkages. It is the most common fibre, constituting 10 to $30 \%$ of the NSP in foods (21).

Though the effects of cellulose on the gut microbiota remains mostly unexplored, some studies are showing the presence of microorganisms in the human intestine capable of fermenting this compound. First studies demonstrated that cellulose degrading bacteria were present only in methane-excreting volunteers (26). However, a study performed in 2010 (27) showed that all participants had cellulose degrading bacteria, but the community differed largely depending on the methane status of volunteers. Methane-excreting subjects had Firmicutes (e.g. Ruminococcus sp.) as dominant cellulose-degrading bacteria, while in nonmethane-excreting volunteers Bacteroidetes (e.g. Bacteroides cellulolysiticus) 
was the predominant phylum of cellulose degrading bacteria (27). The authors proposed that a cross-feeding between hydrogen producing bacteria and methanogens occurs. However, since Bacteroides did not produce hydrogen upon cellulose fermentation, other species may contribute to hydrogen production in the gut (27).

\section{Hemicellulose}

Different from cellulose, hemicelluloses are a heterogeneous group of polymers composed of different sugars which occur often as highly branched chains (21). Besides glucose, it also contains xylose, arabinose, galactose, mannose, rhamnose, glucuronic and/or galacturonic acids (28).

Arabinogalactan is a type of hemicellulose (NSP) and is present in cell walls of plant cells (29). It is densely branched and composed basically of D-galactose and L-arabinose, as well as variable amounts of uronic acids (30). It is part of the human diet, being present in seeds, leaves, roots and fruits, such as carrot, radish, pear, maize, wheat and tomato (29).

\section{Inulin}

Inulin-type fructans are water soluble fructose $(\beta-(2-1)$ fructosyl-fructose linkage) based polysaccharides (degree of polymerization $D P>10$ ) that in plants act as carbohydrates reserve usually stored in bulbs, tubers and tuberous roots, such as wheat, onion, bananas, garlic and chicory (31-33).

\section{Pectins}

Pectin is considered one of the most complex family of polysaccharides in nature because it can be composed of up to 17 different monosaccharides linked by $\sim 20$ different linkages (34).

The basic structure of pectin consists of homogalacturonan (HG), rhamnogalacturonan (RG) I and II, arabinan, galactan, arabinogalactan and xylogalacaturonan (XGA), represented in Figure 2 (35).

$\mathrm{HG}$ is a polymer which contains $\alpha-(1,4)$ linked galacturonic acid residues (GalA), that in turn may be esterified with methanol or are acetylated (34). Pectins can be classified according to their degree of methylation (DM). High methylesterified (HM) pectins have $\mathrm{DM}>50 \%$, whereas low methylesterified (LM) have DM $<50 \%$ (36). Animal studies demonstrated that HM pectins were fermented slower than LM pectins $(37,38)$. This can be a very important characteristic, since normally most of the carbohydrates are fermented in the proximal colon, and therefore the distal colon is depleted of this nutrient, leading to the fermentation of proteins, with consequent negative effects to the host, such as colon cancer (39). 
The RG I backbone is composed of alternating galacturonic acid and rhamnose, where depending of the plant source, rhamnose residues could be substituted with arabinan, galactan and/or arabinogalactan side chains (34).

RG II is considered as a structure within HG, which contains different side chains (34). XGA can be present as a substitute of HG.

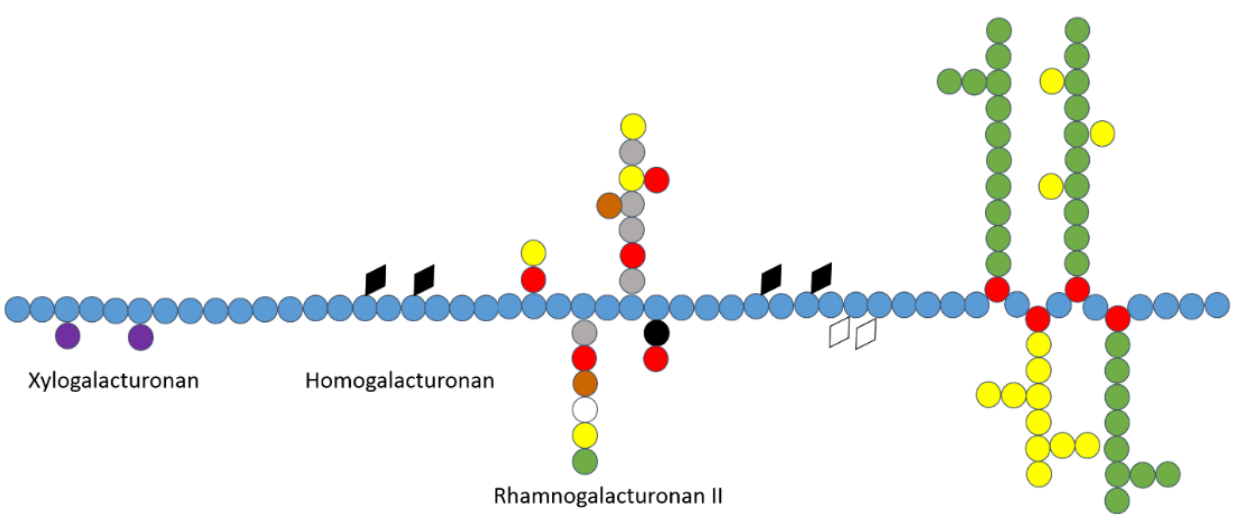

Rhamnogalacturonan I

\begin{tabular}{|lll|}
\hline Galacturonic acid & Rhamnose & xylose \\
Galactose & Apiose & Ketodeoxymannooctulopynosylic acid (KDO) \\
Arabinose & Fucose & Methyl ester \\
& & \\
& & Acetyl ester \\
\hline
\end{tabular}

Figure 2: Schematic representation of the basic structure of pectin. Adapted from $(40,41)$.

An important health effect of pectin relates to the ability to decrease cholesterol levels. The viscous gel formed by pectin binds cholesterol and bile acids and consequently promotes their excretion, reducing reabsorption (42).

Bacterial species able to use pectin for their growth include Faecalibacterium prauznitzii, Eubacterium eligens and Lachnospira pectinoschiza (43).

\section{Food industry by-products - treasures that go to the waste bin}

Processing of raw food products generates residues (waste- or by-products) that are generally underused or even discarded in the environment, being a source of pollution. The discarding of by-products can be viewed as wasting food, since most of them are suitable for human consumption and are full of (micro)nutrients, such as fibres, vitamins, minerals and antioxidants. Additionally, this also represents a waste of resources used in the production process, like water, land and labour (44). 
Paradoxically, wasted food walks hand in hand with the existence of nutritional deficiencies. Many nutrients that are consumed below the recommended amounts are discarded in great amounts. A good illustration for this refers to fibres. A study conducted in the United States demonstrated that the amount of food wasted in a period of 1 year would be enough to fill the gap between the actual and the recommended amount of fibre intake for 206.6 million women (45). Thus, it is recognized that the global food system is not sustainable. At the same time that we have a third of all food produced lost or wasted, billions of people are malnourished or undernourished, whereas other are overweight and obese (46).

"Sustainable diets are protective and respectful of biodiversity and ecosystems, culturally acceptable, accessible, economically fair and affordable; nutritionally adequate, safe and healthy; while optimising natural and human resources" ${ }_{(46)}$

For Meybeck and collaborators, diet can be both the result and the driver for sustainable food systems (46). Therefore, consumers have an important role in guiding the necessary changes towards a more sustainable food system. For that, it is necessary to make them aware of the magnitude of the impact that their food choices can have, and their power to force the food industry into a more responsible and sustainable way of production. This includes the adequate destination of by-products and their optimal use, like including them into the human diet.

\section{Cassava bagasse}

Cassava (Manihot esculenta Crantz) is a crop native to Brazil and it is considered the world's sixth most important tuber, being a source of food and dietary calories for more than 700 million people especially in Latin America, Asia and Africa (47). It has special importance to low income families in the tropical area because this crop is quite tolerant to drought and productive in poor soils (48). In 2009 the consumption of cassava per capita in Brazil was $42.2 \mathrm{~kg} /$ year (49). Cassava can be consumed in natura (after cooking), nevertheless, as it spoils 
easily, the production of cassava flour or cassava starch (tapioca starch) is a manner to conserve it longer (50). However, during cassava flour or starch processing, a solid residue is generated, called bagasse, which contains the fibre material from the root and the remaining starch not extracted during the manufacturing (51).

To produce one ton of starch around $928.6 \mathrm{~kg}$ of humid cassava bagasse is generated, and in Brazil, per year, more than 2 million tons of this humid bagasse is generated (51).

Unfortunately at present, at least in Brazil, there is no process used at industrial scale that utilizes cassava bagasse due the high moisture level (often higher than $80 \%$ ) that impairs transportation because of the large volume, but mainly because the material is highly perishable (52). Therefore, since cassava bagasse does not present a great value in the market, the main limitation of the commercialization and use of this residue is the drying process, considered too expensive and currently not worth to be performed (52).

This residue is thus an industrial waste and it is generally used for animal feeding or even discarded in the environment, thereby considered an ecological hazard (47). Additionally, the discarding of cassava bagasse can be seen as a waste of revenue for the food industry, but especially for small producers, particularly nowadays where the advent of biotechnological approaches is permitting the research for newer applications aiming at value creation and value addition (47).

\section{Orange bagasse}

Brazil is the world's largest producer of oranges and is responsible for $50 \%$ of the total production of orange juice worldwide (53). For every five glasses of orange juice consumed around the world, three are from the Brazilian industry (53). In 2015, Brazil produced more than 16 million tons of oranges (54), of which around $70 \%$ was destined for juice production $(53,55)$. Therefore, the generation of residues (bagasse) is significant, since $50 \%$ of the total fruit is composed of peel, albedo, membranes and seeds (53).

Orange bagasse can be used for animal feeding (low-value product), but it is also a common practice to discard it into the environment without any type of care, thus being a source of pollution (56). Additionally, some of the peel is used for pectin production (high-value product), but the market demand for pectin is low compared to the total amount of pectin that could be produced from the worldwide supply of citrus by-product (57).

Hence, there is a demand for new approaches to make use of the large amount of residues generated during juice extraction. This could bring benefits for the food industry, environment and consumers. Here, the strategy is to search for health 
benefits (especially regarding intestinal health) that the consumption of orange waste could bring, when incorporated into a human diet.

\section{Passion fruit peel}

Passion fruit is a tropical plant native to Brazil, which is the global largest producer. In 2015, almost 700 thousand tons were produced, of which $40 \%$ is used by the food industry to produce juice (54).

Around $40-60 \%$ of the total fruit mass of passion fruit corresponds to residue (peel and seeds) and is currently discarded (58).

In Brazil, clinical research is focused on the role of passion fruit peel in decreasing blood sugar in diabetics patients, with some controversial results $(59,60)$. However, there is a lack of knowledge regarding its potential prebiotic effect and the relationship with obesity, which is the focus of this thesis.

\section{The ever-increasing problem of obesity}

According to the World Health Organization (WHO), obesity has more than doubled since 1980 and in 2014, more than 1.9 billion adults were overweight (body mass index [BMI] $>25 \mathrm{~kg} / \mathrm{m}^{2}$ ), of which 600 million were obese (BMI $>30$ $\mathrm{kg} / \mathrm{m}^{2}$ ) (61). It is considered a major health issue since obesity has a positive association with risk of mortality and besides that, it usually results in serious complications such as type 2 diabetes, cardiovascular disease and diverse types of cancer (61).

Although apparently simple, healthy diet and exercise are not fully effective in tackling obesity, as can be proven by its increased incidence (61). The issue is that the implementation of these two elements impose major changes in people's lifestyle and for most of individuals, these changes are not easy to maintain on the long-term. Furthermore, obesity is a multifactorial disease that results from a complex interplay between several factors such as genetics, life style, dietary habits, and obesogenic environment (62). Therefore, it is likely that a combination of other approaches to revert obesity is necessary.

Interestingly, the gut microbiota has been suggested as an environmental factor that, together with the above mentioned items, can contribute to the onset of obesity (Figure 3) (63).

It is known that lean and obese people have a different microbiota composition (64). However, currently the great debate is whether this difference is the cause or consequence of obesity. 


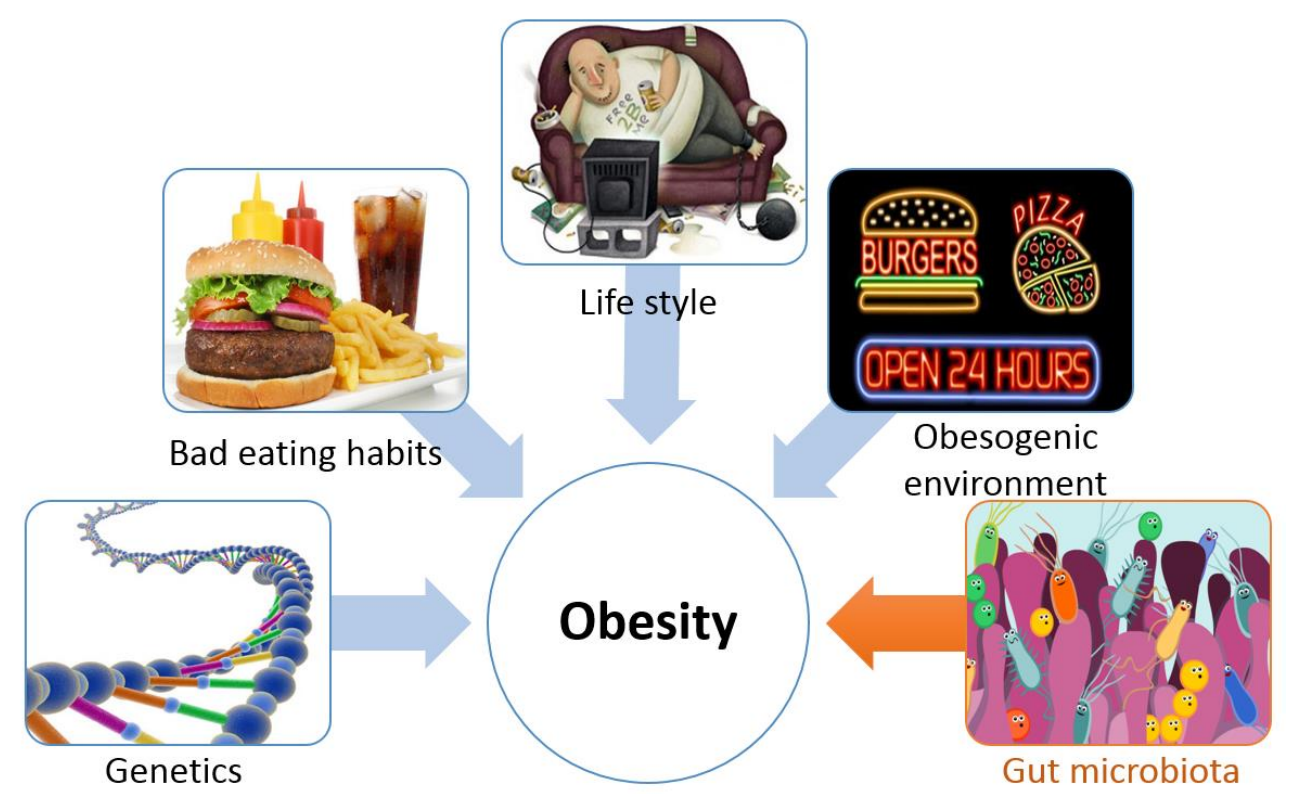

Figure 3: Diverse factors that contribute to obesity, such as genetics, bad eating habits, life style, obesogenic environment and, as described recently by the literature, gut microbiota.

\section{Microbiota composition in lean and obese individuals - no consensus after more than a decade of studies}

Perhaps the most important pioneering studies about the relationship between the gut microbiota and obesity are from the Gordon group, from Washington University in St. Louis. A set of elegant studies demonstrated that the gut microbiota had an influence on the host energy metabolism - at least in rodents (63-65), and more importantly, raised several questions that still drive research in this field.

A comparison between germ free mice (GF) and conventionally raised animals (CONV-R) demonstrated that CONV-R mice had $42 \%$ more total body fat, despite a lower daily consumption of the standard diet (63). Next, conventionalization of GF mice with microbiota from CONV-R animals caused an increase of 57\% in total body fat. The authors proposed two mechanisms for the gut microbiota promoting weight gain. First, gut microbiota has the necessary enzymes to process indigestible polysaccharides and therefore provide the host with extra energy via their breakdown. 
The extra energy can come from the released monosaccharides that can be absorbed by intestinal tissue or via the production of SCFA (through fermentation of these monosaccharides) that will also be absorbed by intestinal tissue. These two elements will increase hepatic lipogenesis and consequently increase triglycerides storage in adipocytes (Figure 4) (63).

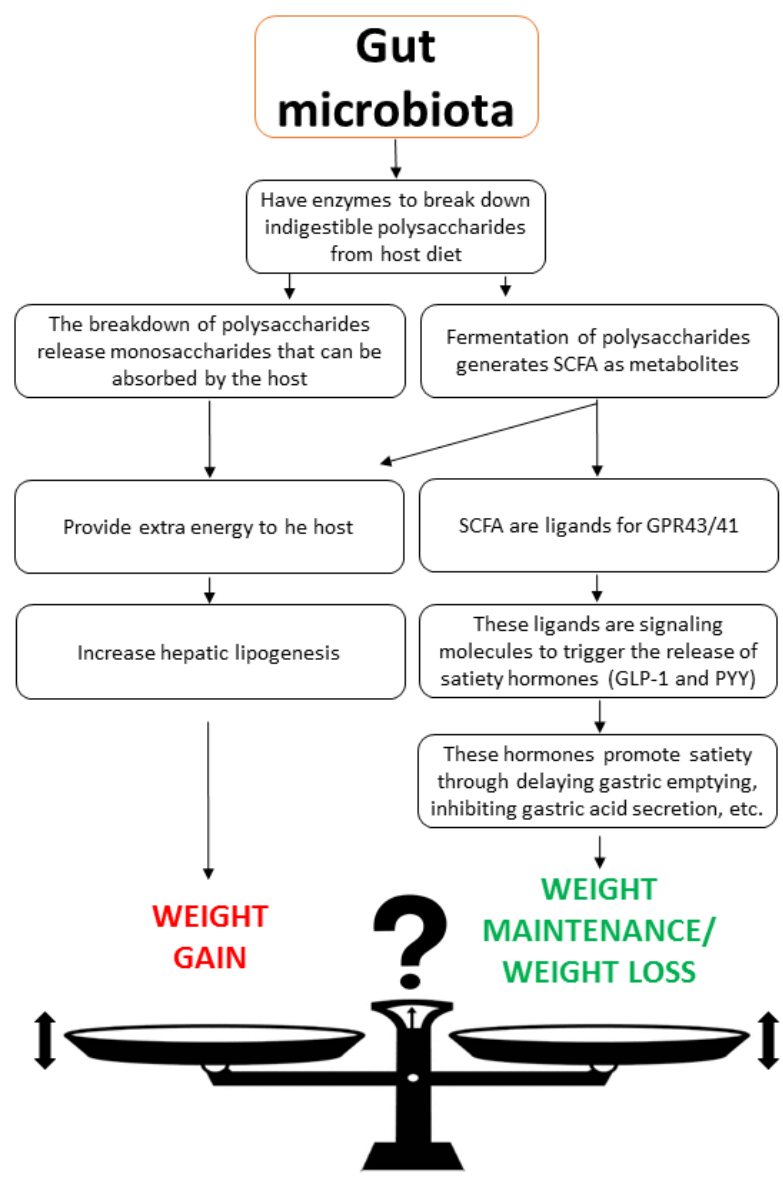

Figure 4: Gut microbiota and their relationship with energy balance. Adapted from Backhed 2004 (63).

The second mechanism relies on the suppression of Fiaf (Fasting-induced adipose factor, also known as Angiopoietin-like protein 4) by the gut microbiota. Fiaf is an inhibitor of LPL (Lipoprotein Lipase) and it is produced by several tissues, including intestinal tissue. Its suppression allows the increase in LPL activity. In turn, an increased LPL activity leads to an increased cellular uptake of free fatty acids and increase in triglycerides storage in adipocytes (63). The authors concluded that ancient humans that were subjected to fluctuating 
availability of food benefited from the extra energy provided by the gut microbiota. However, the current situation in which most Western populations are living, with easy access to high density caloric food, is providing detrimental effects. In the authors opinion, depending on lifestyle conditions, differences in gut microbiota composition could be either protective or predispose individuals to develop obesity (63).

A second set of studies revealed that the microbiota from obese mice had 50\% reduction in Bacteroidetes and increased proportion of Firmicutes when compared with lean mice. The authors generated two hypotheses: the increased ratio of Firmicutes/Bacteroidetes in obese mice may help promote adiposity, or this could be an adaptive response from the host to limit energy uptake/storage, since Bacteroidetes has a vast variety of species known by its capacity to degrade polysaccharides (65), which would contribute to energy that becomes available to the host, through the monosaccharides directly, but more likely through the SCFA produced from these by the microbes.

They compared whether the difference in the ratio of Firmicutes/Bacteroidetes in lean and obese mice would have an effect on the metabolic potential of the gut microbiota regarding energy harvesting (66). Through metagenomics analyses it was verified that the obese microbiome was enriched with genes encoding several different enzymes involved in the initial breakdown of indigestible polysaccharides, when compared with lean animals (66). Additionally, to test if the microbiota from obese animals could transfer the capacity to harvest more energy from indigestible carbohydrates, microbiota transplantation experiments were performed (66). Interestingly, mice that received a microbiota from obese animals showed an increase in body fat (66).

These set of studies by the Gordon group intrigued the academic community, especially regarding whether such findings could be translated/replicated into humans.

This however was not always the case. A study performed with 98 human subjects encompassing lean, overweight and obese individuals in fact presented the opposite result regarding the ratio of Firmicutes/Bacteroidetes (67). Interestingly, the ratio changed in favour of the Bacteroidetes phylum in overweight and obese individuals. Concentrations of faecal SCFA were also analysed, and obese individuals had higher concentration of these metabolites in their faeces when compared with lean volunteers. According to the authors, the amount of SCFA produced could be more important in the relationship of gut microbiota and obesity than the ratio of Firmicutes to Bacteroidetes. However, they neglected the fact that a higher excretion of SCFA does not necessarily mean 
a higher SCFA production. If production is the same, but uptake is lower, excretion will be higher. In this case, energy extraction would be reduced.

A recent study with young human individuals found that the ratio Firmicutes to Bacteroidetes was positively associated with BMI, and plasma levels of SCFA was associated with body fat partitioning and hepatic lipogenesis. When using an in vitro assay to test the fermentation ability of gut microbiota from the lean and obese groups, researchers found that the obese microbiota had an increased capability to ferment the same amount of fructose as the lean microbiota (68), although it should be realized that this is not a substrate that would normally be present in the colon.

The above-mentioned studies are just a few examples to indicate that there still is a paradox regarding the relationship of certain groups of bacteria with obesity. Currently the evidence on the link between the Bacteroidetes to Firmicutes ratio and weight is clearly conflicting and different subgroups within phyla may behave differently in relation to obesity (69), indicating the necessity to further investigate this field.

New studies are focusing on specific bacterial species and/or groups of bacteria and their relationship with obesity. Currently, Akkermansia muciniphila, a mucin degrading species from the Verrucomicrobia phylum, is calling attention. It is being suggested as a biomarker for intestinal health, since its abundance is inversely correlated with various intestinal disorders, such as inflammatory bowel disease (IBD) (70).

A study with pregnant women $(\mathrm{n}=50)$ showed that A. muciniphila and Bifidobacterium numbers were higher in women with normal weight gain compared with women that had an excessive weight gain during this period (71). Another study with overweight and obese individuals $(n=49)$ showed that the abundance of $A$. muciniphila was associated with a healthier metabolic status in this population (72). Interestingly, after a caloric restriction diet, individuals that had higher A. muciniphila at the baseline demonstrated a better improvement in insulin sensitivity markers, body composition and blood lipids (72).

However, research performed with 345 Chinese type 2 diabetic (T2D) patients and nondiabetic controls revealed that A. muciniphila was enriched in T2D patients stool samples (73), showing some discrepancy regarding the relationship between $A$. muciniphila and health status. Nevertheless, this discrepancy could be due to differences in study design, ethnicity, and age, among others.

With the attempt to find the physiological and mechanistic role of this bacterium during obesity and metabolic disorders, Everard et al. (2013) performed a study 
with obese and diabetic mice. First, they found that obese and diabetic mice presented lower abundance of $A$. muciniphila. Second, when treating these animals with a prebiotic (oligofructose), the abundance of $A$. muciniphila was reestablished. Finally, administration of $A$. muciniphila to mice reversed high fat diet metabolic disorders, such as metabolic endotoxemia (74). The authors proposed as possible mechanism the improvement of the mucus layer by $A$. muciniphila (74), which would decrease intestinal permeability and its deleterious consequences. Importantly, it has been suggested that microbes colonizing the mucus layer can protect the host against intestinal pathogens (70).

To test the hypothesis that variation in the gut microbiota (at genus and species level) could predict which adult individuals would be at increased risk to develop obesity-related metabolic disorders, Le Chatelier and collaborators (2013) assessed the number of gut bacterial genes from 292 obese and non-obese individuals (75). A bimodal distribution of bacterial genes was found, and individuals could be separated into two groups: "low gene counts" (LGC) and "high gene counts" (HGC) (75). This difference in gene counts was an indication that these two groups would have different microbial communities in their gut. Thus, a deeper analysis was performed and the researchers found that HGC individuals had a higher prevalence of anti-inflammatory species, like Faecalibacterium prausnitzii, as well as the genera Akkermansia and Bifidobacterium, whereas LGC individuals harboured more potentially proinflammatory bacteria, such as Bacteroides and Ruminococcus gnavus, normally associated with IBD (75). Genomic analysis revealed a greater genetic potential for production of metabolites with presumptive deleterious effects on the host, such as pro-carcinogens, in LGC individuals (75). In contrast, the HGC group displayed a vast genetic potential for production of organic acids, such as butyrate and propionate (75). Importantly, the LGC group was composed of a significantly higher proportion of obese participants with metabolic disturbances that could put them at increased risk for T2D and cardiovascular disorders (75). Additionally, it was verified that the gut microbiota composition from lean and obese people differed less when compared with LCG and HGC (75), demonstrating that there is still a long way to go regarding a consensus about gut microbiota and obesity. However, this difference in gene count could explain why some obese individuals are considered "healthy obese", while others are more prone to a worse prognostic regarding their obesity and its deleterious consequences.

Following this rational, Sommer et al. analysed the gut microbiota from brown bears, which have two life cycles during the year, one characterized by an intense eating and weight gain (summer) and other characterized by hypometabolic 
fasting (winter) (76). The important issue is that despite a great fat accumulation during summer, they remain metabolically healthy, and researchers believe that brown bears could constitute an important model for healthy obesity (76). When comparing gut microbiota from summer and winter, the authors found that microbiota from summer had higher microbial taxa and was more diverse, probably due to a diverse diet during this period. Summer microbiota was dominated by Proteobacteria, Firmicutes and Actinobacteria phyla, while in winter the microbiota was increased by Bacteroidetes while Firmicutes and Actinobacteria were less abundant (76). Serum levels of cholesterol, triglycerides and free cholesterol were higher during the winter, suggesting that during the hibernation period, lipids are used as a survival strategy (76). Next, researchers colonized GF mice with bear microbiota from summer and winter, in order to test whether the seasonal differences in bear microbiota could affect host physiology. Interestingly, some features could be transmitted to mice, such as higher weight and fat gain in mice receiving microbiota from summer period. However, there was no difference regarding glucose metabolism between mice from the two groups (76), suggesting the role of the gut microbiota in healthy obesity.

\section{Short chain fatty acids (SCFA) and their relationship with obesity}

Fermentation is the process through which anaerobic bacteria and yeasts degrade dietary compounds in the absence of oxygen in order to obtain energy for growth and the maintenance of cellular function (77).

In the colon, carbohydrates are fermented to SCFA, mainly acetate, propionate and butyrate (78). The Bacteroidetes phylum contains species that produce mainly acetate and propionate, while species that belong to Firmicutes are responsible for the production of mostly butyrate (79). Butyrate is the major fuel for colonocytes, supplying $60-70 \%$ of energy needs (13). Around $90 \%$ of propionate is used by the liver as precursor of hepatic gluconeogenesis and therefore converted into glucose (79). Acetate is mainly found in peripheral circulation and consequently can act in different tissues (79).

SCFA can contribute to about 5-15\% of the total caloric requirements of humans (80). Knowing that even the consumption of $1 \%$ more than the daily energy needs can lead to weight gain on the long-term, the amount of energy released by the gut microbiota can have important consequences for body weight (81). However, at the same time that the fermentation of carbohydrates by the gut microbiota provides extra energy to the host, these same SCFA are ligands of free 
fatty acid receptor (FFAR) 2 and 3 (formerly called G-protein coupled receptors (GPCR) 43 and 41, respectively). FFAR 2 and 3 are responsible for triggering the release of satiety hormones GLP-1 and PYY by L-cells (Figure 4) (82). Another in vitro study demonstrated that butyrate increased the expression as well as release of PYY in epithelial cells (12). PYY inhibits gastric emptying and food intake, whereas GLP-1 also stimulates insulin secretion $(83,84)$. Increasing transit time of ingested food through the gastrointestinal tract prolongs the stimulation of mechanic/chemical receptors responsible for signalling satiety after food intake (79).

In vitro studies have verified that acetate and propionate inhibited intracellular lipolysis, and thus could have an effect on reducing fat accumulation (13). On the other hand, incubation of murine and porcine pre-adipocytes with acetate, propionate and butyrate stimulated their differentiation, indicating proadipogenic properties of SCFA (13). Additionally, acetate production after lactulose ingestion by overweight subjects resulted in short-term decrease in free fatty acid level and glycerol turnover related to a decrease of lipolysis, which is believed to help in preventing insulin resistance (12).

These findings suggest an important role of microbial metabolites in energy metabolism, food intake, satiety and satiation. However, more studies are necessary in order to reveal the mechanisms behind how the gut microbiota and SCFA influence obesity and establish the final picture of this complex relationship.

\section{In vitro models of the gastrointestinal tract, the tool for mechanistic insight}

Although in vivo studies with humans are the ultimate method to evaluate the effects of dietary compounds on human health, they are costly, highly invasive and restrictive due to ethical reasons. As an alternative, in vitro models that accurately simulate the gastrointestinal (GI) tract emerge as an essential tool especially to test novel foods, with several advantages that go from the reduction in use of animals in experiments, until the possibility of sampling different segments of the GI tract and closely monitoring the fate of degradation and fermentation of dietary fibres, for instance.

Here the two in vitro models of the GI tract used in this thesis are described: TIM1 that simulates the upper GI tract (stomach, duodenum, jejunum and ileum) and TIM-2, which simulates the proximal colon. 


\section{TIM-1 system}

TIM-1 is a multi-compartmental, computer-controlled, dynamic in vitro model that simulates the stomach and small intestine. It consists of four compartments that simulate the stomach, duodenum, jejunum and ileum, which are connected by computer controlled valves (Figure 5) (85).

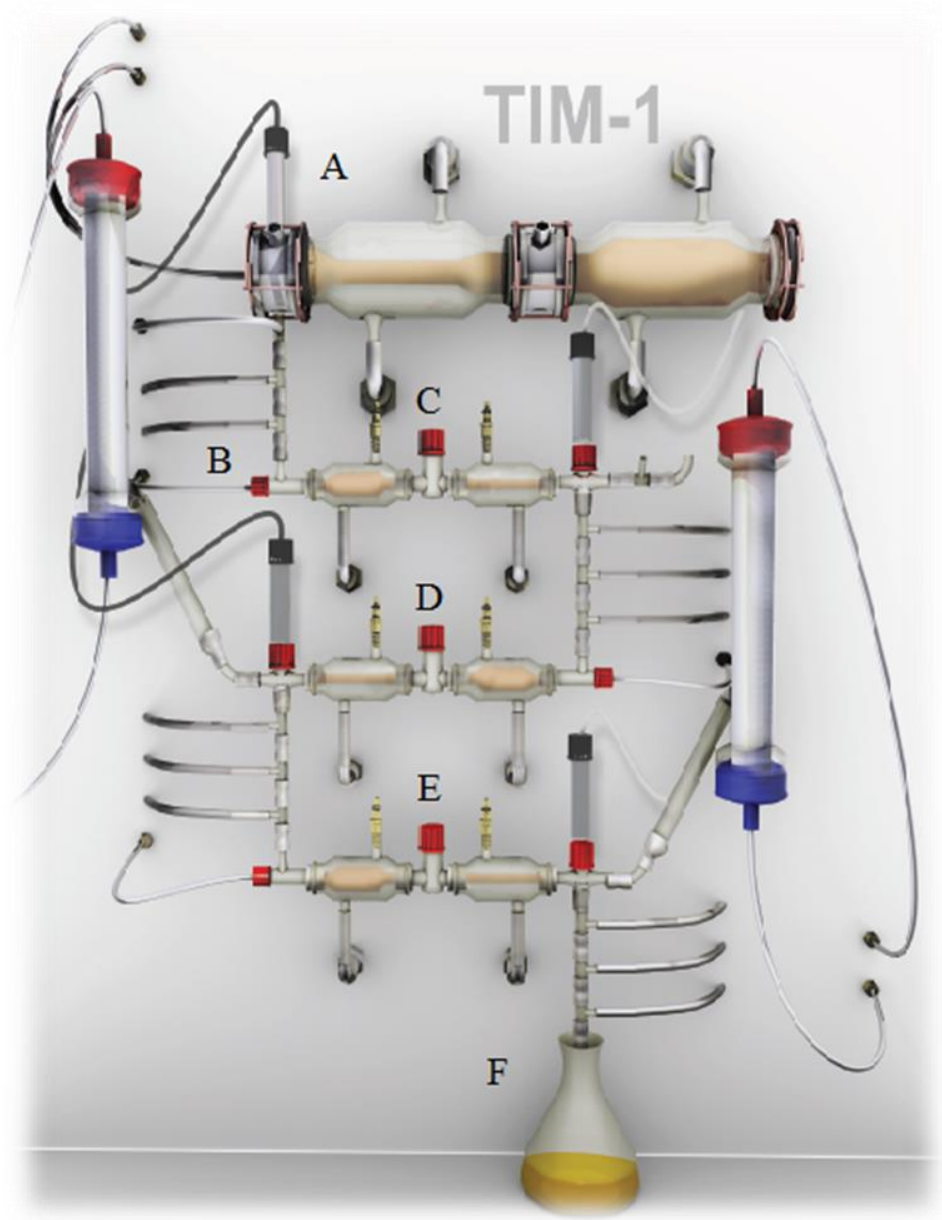

Figure 5: Schematic diagram of the stomach and small intestine model (TIM-1). A = stomach compartment; $\mathrm{B}=$ pyloric sphincter; $\mathrm{C}=$ duodenum compartment; $\mathrm{D}=$ jejunum compartment; $\mathrm{E}$ = ileum compartment; $\mathrm{F}$ = ileal efflux.

The temperature is kept at body temperature $\left(37^{\circ} \mathrm{C}\right.$ for humans). The secretion of fluids and enzymes in each compartment is set up to mimic the physiological conditions of healthy humans. In this thesis these parameters for adults were used, but also parameters for babies and elderly are available. Peristaltic movements are mimicked through the contraction of the flexible walls, which is also responsible for controlling gastric emptying and food transit (85). 
The contents of the jejunum and ileum compartments are dialyzed continuously using hollow fibre semi-permeable membranes, resembling the absorption of the digested products such as sugar, oligosaccharides, amino acids and small peptides by intestinal cells (85).

TIM-1 is an important tool that enables the characterization of test products regarding for instance their digestibility. Here, this system was essentially used to evaluate the digestibility (starch and protein fractions) of the by-products of Brazilian food-industry used in this thesis, and to assess the fate of absorption of the digestible components.

\section{TIM-2 system}

Intestinal models have also risen as an attractive alternative to study the metabolic and ecological behaviour of the gut microbiota (86).

TIM-2 (TNO intestinal model of the large intestine) (Figure 6) is a state-of-theart computer-controlled, dynamic system that simulates several features of the human large intestine.

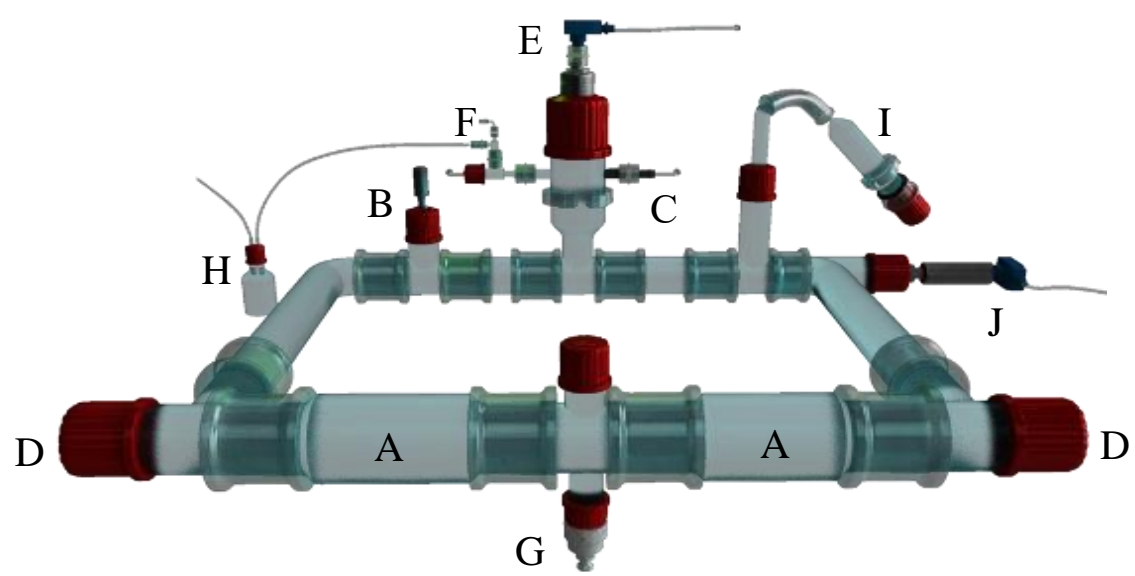

Figure 6: TIM-2 system. $A=$ peristaltic compartments with a dialysis membrane inside; $\mathrm{B}=\mathrm{pH}$ sensor, $\mathrm{C}=\mathrm{NaOH}$ secretion; $\mathrm{D}=$ dialysate system; $\mathrm{E}=$ level sensor; $\mathrm{F}=$ gaseous $\mathrm{N}_{2}$ inlet; $\mathrm{G}=$ sampling port; $\mathrm{H}=$ gas outlet; $\mathrm{I}=$ feeding syringe with test compound; $\mathrm{J}=$ temperature sensor. Adapted from (87).

The temperature is kept at $37^{\circ} \mathrm{C}$; the $\mathrm{pH}$ is constantly checked using a $\mathrm{pH}$ sensor and maintained at 5.8 through secretion of sodium hydroxide; constant flushing of gaseous nitrogen creates an anaerobic environment; water and fermentation products are removed through a dialysis system; and the simulation of peristaltic movements is responsible for mixing and transport of intestinal contents (86). 
Water and fermentation products are constantly removed through the dialysis system. Thus, physiological concentrations of small molecules, such as electrolytes and microbial metabolites, are maintained and product inhibition of enzyme activities and inhibition of growth of microorganisms as a result of accumulation of microbial metabolites is prevented. It was previously demonstrated that TIM-2 allows for the growth of a highly active and dense microbiota, comparable to that found in the human proximal colon (88).

However, it is important to be aware of the limitations of in vitro models. The lack of host cells that limits the microbe-host interaction is an example. However, taking the limitations into account, this system is a useful tool to study for instance, indigestible/undigested components and their effect on microbial metabolism and ecology in the lumen of the large intestine (86) and can be used to study hypotheses with respect to the role of the microbiota in obesity, energy extraction and the fate of polysaccharide fermentation (89).

TIM-2 was the main tool used in this thesis, with the objective to verify the potential prebiotic effect of the different by-products tested. In addition, it was also used to assess possible differences in fermentability (microbiota composition and production of microbial metabolites) when using a microbiota from lean or obese individuals. 


\section{Aims and outline of the thesis}

As previously described, the discard of by-products generated by the food industry, containing valuable ingredients such as fibre and antioxidants, is a serious problem that urges for a solution. At the same time, obesity has emerged as a disease that globally affects millions of people, characterized by among others, an imbalanced diet and dysbiosis as important factors that contribute to the maintenance of this problem. In this context, the use of prebiotics as an additional tool to tackle obesity is a promising field, especially because the use of prebiotics is a non-invasive and non-expensive approach.

The aim of this thesis was to assess the potential prebiotic effect(s) of a number of (Brazilian) food by-products using an in vitro model of the proximal colon, either with microbiota from healthy individuals in some chapters, and lean or obese individuals in other chapters.

The first original scientific chapter (Chapter 2), describes the chemical characterization of eight different food by-products (three cassava bagasses, two orange bagasses and three passion fruit peels) and their alcohol insoluble solids (AIS) fraction, which can be seen to represent the indigestible fractions. In the same chapter, digestibility of all three cassava bagasses, and one sample of orange bagasse and passion fruit peel were evaluated in an in vitro model that simulates the upper gastrointestinal tract (TIM-1).

Chapter 3 describes the first set of experiments performed to test in vitro (in TIM-2) the potential prebiotic effect of one sample of cassava bagasse when using microbiota originated from lean or obese individuals. The fermentation profile - microbiota composition and production of SCFA, BCFA, lactate and ammonia were compared between the so called "lean and obese microbiota".

In Chapter 4 we included all food by-products characterized in Chapter 2, and continued with the evaluation of the potential prebiotic effects of them, using the same in vitro approach as in Chapter 3 - TIM-2. The in vitro model was inoculated with a faecal slurry from lean or obese individuals and results of microbiota composition, SCFA, BCFA and organic acids were compared between these two groups.

After the evaluation of the effects that each by-product had in the microbial dynamics, the isolated indigestible fibres - AIS fractions of orange bagasses and passion fruit peels were used in in vitro fermentation experiments using 
microbiota from healthy individuals (Chapter 5). This study also describes the fate of these fibres during fermentation, which was performed through the analyses of intermediate degradation products. The objective of such analyses was to investigate the kinetics of bacterial utilization of the different monosaccharides present in these polysaccharides.

In Chapter 6 we proceeded with the evaluation of the differences on the fermentation profiles using microbiota derived from lean and obese volunteers. Two fibres were used - inulin and arabinogalactan, and compositional changes and metabolite production were assessed.

Chapter $\mathbf{7}$ focuses on the structure-function relationship of various pectins by studying their potential to modulate the gut microbiota. The dynamics of microbiota composition and activity was assessed during fermentation of nine structurally diverse pectins extracted from different food by-products, and a pectic derivative rhamnogalacturonan I. This study was performed in TIM-2 that was inoculated with a microbiota from healthy individuals.

A general discussion about the results presented in this thesis is provided in Chapter 8. Main findings and new insights obtained are discussed.

The thesis finishes with a summary and a validation section. 


\section{References}

1. Insel P, Ross D, McMahon K. Discovering nutrition. Jones \& Bartlett Publishers; 2013.

2. Geissler C, Powers H. Human nutrition. Oxford University Press; 2017.

3. de Graaf AA, Venema K. Gaining insight into microbial physiology in the large intestine: a special role for stable isotopes. Adv Microb Physiol. England; 2008;53:73-168.

4. O'Hara AM, Shanahan F. The gut flora as a forgotten organ. EMBO Rep. European Molecular Biology Organization; 2006;7(7):688-93.

5. Thursby E, Juge N. Introduction to the human gut microbiota. Biochem J. England; 2017 May;474(11):1823-36.

6. Rowland I, Gibson G, Heinken A, Scott K, Swann J, Thiele I, et al. Gut microbiota functions: metabolism of nutrients and other food components. Eur J Nutr. Germany; 2017 Apr.

7. Cani PD, Delzenne NM. The role of the gut microbiota in energy metabolism and metabolic disease. Curr Pharm Des. 2009;15(13):154658.

8. Aguirre $\mathrm{M}$, Venema $\mathrm{K}$. The art of targeting gut microbiota for tackling human obesity. Genes Nutr. Berlin/Heidelberg: Springer Berlin Heidelberg; 2015 Jul 20;10(4):20.

9. Kovatcheva-Datchary P, Zoetendal EG, Venema K, de Vos WM, Smidt H. Tools for the tract: understanding the functionality of the gastrointestinal tract. Therap Adv Gastroenterol. England; 2009 Jul;2(4):9-22.

10. Hamer HM, De Preter V, Windey K, Verbeke K. Functional analysis of colonic bacterial metabolism: relevant to health? Am J Physiol Gastrointest Liver Physiol. 2012;302(1):G1-9.

11. Rose DJ, Venema K, Keshavarzian A, Hamaker BR. Starch-entrapped microspheres show a beneficial fermentation profile and decrease in potentially harmful bacteria during in vitro fermentation in faecal microbiota obtained from patients with inflammatory bowel disease. Br J Nutr. 2010;103(10):1514-24.

12. Hamer HM, Jonkers D, Venema K, Vanhoutvin S, Troost FJ, Brummer R-J. Review article: the role of butyrate on colonic function. Aliment Pharmacol Ther. England; 2008 Jan;27(2):104-19.

13. Canfora EE, Jocken JW, Blaak EE. Short-chain fatty acids in control of body weight and insulin sensitivity. Nat Rev Endocrinol. Nature Publishing Group; 2015;11(10):577-91.

14. Scott KP, Gratz SW, Sheridan PO, Flint HJ, Duncan SH. The influence of diet on the gut microbiota. Pharmacol Res. Elsevier Ltd; 2013;69(1):52-60.

15. Martens EC, Kelly AG, Tauzin AS, Brumer H. The devil lies in the details: How variations in polysaccharide fine-structure impact the physiology and evolution of gut microbes. J Mol Biol. Elsevier B.V.; 2014;426(23):3851-65.

16. Stephen AM, Champ MM-J, Cloran SJ, Fleith M, van Lieshout L, Mejborn H, et al. Dietary fibre in Europe: current state of knowledge on definitions, 
sources, recommendations, intakes and relationships to health. Nutr Res Rev. Cambridge University Press; 2017 Jul 5;1-42.

17. Gibson GR, Hutkins R, Sanders ME, Prescott SL, Reimer RA, Salminen SJ, et al. Expert consensus document: The International Scientific Association for Probiotics and Prebiotics (ISAPP) consensus statement on the definition and scope of prebiotics. Nat Rev Gastroenterol Hepatol. England; 2017 Aug;14(8):491-502.

18. Macfarlane S, Macfarlane GT, Cummings JH. Review article: Prebiotics in the gastrointestinal tract. Aliment Pharmacol Ther. 2006;24(5):701-14.

19. Macfarlane GT, Macfarlane S. Bacteria, colonic fermentation, and gastrointestinal health. J AOAC Int. United States; 2012;95(1):50-60.

20. Jonathan MC. Monitoring the degradation of individual dietary fibres in pig models. Doctoral dissertation. Wageningen University; 2013. 184 p.

21. Cummings JH, Stephen AM. Carbohydrate terminology and classification. Eur J Clin Nutr. 2007;61 Suppl 1:S5-18.

22. Sajilata MG, Singhal RS, Kulkarni PR. Resistant starch-a review. Compr Rev food Sci food Saf. Wiley Online Library; 2006;5(1):1-17.

23. Resistant Starch. Proceedings for the 2nd plenary meeting of EURESTA: European FLAIR Concerted Action No. 11 on physiological implications of the consumption of resistant starch in man. Crete, 29 May-2 June 1991. In: European journal of clinical nutrition. England; 1992. p. S1-148.

24. Martinez I, Kim J, Duffy PR, Schlegel VL, Walter J. Resistant starches types 2 and 4 have differential effects on the composition of the fecal microbiota in human subjects. PLoS One. United States; 2010 Nov;5(11):e15046.

25. Salonen A, Lahti L, Salojarvi J, Holtrop G, Korpela K, Duncan SH, et al. Impact of diet and individual variation on intestinal microbiota composition and fermentation products in obese men. ISME J. England; 2014 Nov;8(11):2218-30.

26. Robert C, Bernalier-Donadille A. The cellulolytic microflora of the human colon: evidence of microcrystalline cellulose-degrading bacteria in methane-excreting subjects. FEMS Microbiol Ecol. England; 2003 Oct;46(1):81-9.

27. Chassard C, Delmas E, Robert C, Bernalier-Donadille A. The cellulosedegrading microbial community of the human gut varies according to the presence or absence of methanogens. FEMS Microbiol Ecol. England; 2010 Oct;74(1):205-13.

28. Gray J. Dietary fibre. ILSI Europe Concise Monograph Series. 2006. 1-44 p.

29. Dion C, Chappuis E, Ripoll C. Does larch arabinogalactan enhance immune function? A review of mechanistic and clinical trials. Nutr Metab (Lond). England; 2016;13:28.

30. Moschini R, Gini F, Cappiello M, Balestri F, Falcone G, Boldrini E, et al. Interaction of arabinogalactan with mucins. Int J Biol Macromol. 2014;67:446-51.

31. Apolinário AC, de Lima Damasceno BPG, de Macêdo Beltrão NE, Pessoa A, Converti A, da Silva JA. Inulin-type fructans: A review on different aspects 
of biochemical and pharmaceutical technology. Carbohydr Polym. 2014;101:368-78.

32. Fu Y-P, Li L-X, Zhang B-Z, Paulsen BS, Yin Z-Q, Huang C, et al. Characterization and prebiotic activity in vitro of inulin-type fructan from Codonopsis pilosula roots. Carbohydr Polym. 2018;193:212-20.

33. Carlson JL, Erickson JM, Hess JM, Gould TJ, Slavin JL. Prebiotic dietary fiber and gut health: comparing the in vitro fermentations of beta-glucan, inulin and xylooligosaccharide. Nutrients. Switzerland; 2017 Dec;9(12).

34. Voragen AGJ, Coenen G-J, Verhoef RP, Schols HA. Pectin, a versatile polysaccharide present in plant cell walls. Struct Chem. Springer; 2009;20(2):263.

35. Ramasamy US. The role of soluble and insoluble fibers during fermentation of Chicory root pulp. Wageningen University; 2014.

36. Remoroza C. Enzymatic fingerprinting and modification of acetylated pectins. Doctoral dissertation. Wageningen University; 2014.

37. Tian L, Scholte J, Borewicz K, van den Bogert B, Smidt H, Scheurink AJW, et al. Effects of pectin supplementation on the fermentation patterns of different structural carbohydrates in rats. Mol Nutr Food Res. Germany; 2016 Oct;60(10):2256-66.

38. Dongowski G, Lorenz A, Proll J. The degree of methylation influences the degradation of pectin in the intestinal tract of rats and in vitro. J Nutr. United States; 2002 Jul;132(7):1935-44.

39. den Besten G, van Eunen K, Groen AK, Venema K, Reijngoud DJ, Bakker $\mathrm{BM}$. The role of short-chain fatty acids in the interplay between diet, gut microbiota, and host energy metabolism. J Lipid Res. 2013;54(9):232540.

40. Pedrolli DB, Monteiro AC, Gomes E, Carmona EC. Pectin and pectinases: production, characterization and industrial application of microbial pectinolytic enzymes. Open Biotechnol J. 2009;9-18.

41. Rösch C. In vitro fermentation and immunomodulating characteristics of dietary fibres. Wageningen University; 2016.

42. Naqash F, Masoodi FA, Rather SA, Wani SM, Gani A. Emerging concepts in the nutraceutical and functional properties of pectin-A Review. Carbohydr Polym. 2017;168(Supplement C):227-39.

43. Flint HJ, Scott KP, Duncan SH, Louis P, Forano E. Microbial degradation of complex carbohydrates in the gut. Vol. 3, Gut Microbes. 2012. p. 289-306.

44. Food and Agriculture Organization of the United Nations. Global food losses and food waste: Extent, causes and prevention. Rome; 2011.

45. Spiker ML, Hiza HAB, Siddiqi SM, Neff RA. Wasted food, wasted nutrients: nutrient loss from wasted food in the united states and comparison to gaps in dietary intake. J Acad Nutr Diet. United States; 2017 Jul;117(7):1031-1040.e22.

46. Meybeck A, Gitz V. Sustainable diets within sustainable food systems. Proc Nutr Soc. England; 2017 Feb;76(1):1-11.

47. Pandey A, Soccol CR, Nigam P, Soccol VT, Vandenberghe LPS, Mohan R. 
Biotechnological potential of agro-industrial residues. II: cassava bagasse. Bioresour Technol. 2000;74(1):81-7.

48. Food and Agriculture Organization of the United Nations. Cassava. 2017.

49. IBGE. Antropometria e estado nutricional de crianças, adolescentes e adultos no Brasil [Internet]. Vol. 2012. 2009. Available from: http://www.ibge.gov.br/home/presidencia/noticias/noticia_visualiza.p hp?id_noticia=1699\&id_pagina=1/

50. Cardoso EMR, Hühn S, Junior J de DN. Processo industrial para beneficiamento da macaxeira. Novos Cad NAEA. Pará; 1999;2(2):177-84.

51. Fiorda FA, Soares Júnior MS, da Silva FA, Fontinelle Souto LR, Eiras Grossmann MV. Farinha de bagaço de mandioca: Aproveitamento de subproduto e comparação com fécula de mandioca. Pesqui Agropecu Trop. 2013;43(4):408-16.

52. Jasko AC, Andrade J de, Padilha L, Pauli RB de, Quast LB, Schnitzler E, et al. Caracterização físico-química de bagaço de mandioca in natura e após tratamento hidrolítico. Rev Bras Tecnol Agroindustrial. Ponta Grossa; 2011;5:427-41.

53. Neves MF, Trombin VG, Milan P, Lopes FF, Cressoni F, Kalaki R. O retrato da citricultura brasileira. Ribeirão Preto: CitrusBR. 2010.

54. IBGE. Produção agrícola municipal. Vol. 42. Rio de Janeiro; 2015.

55. Citrus BR. Estimativa de safras. 2016. [cited 2017 Jun 30]. Available from: http://www.citrusbr.com/safras/

56. Angel Siles Lopez J, Li Q, Thompson IP. Biorefinery of waste orange peel. Crit Rev Biotechnol. England; 2010 Mar;30(1):63-9.

57. Manderson K, Pinart M, Tuohy KM, Grace WE, Hotchkiss AT, Widmer W, et al. In vitro determination of prebiotic properties of oligosaccharides derived from an orange juice manufacturing by-product stream. Appl Environ Microbiol. 2005;71(12):8383-9.

58. Oliveira LF de, Nascimento MRF, Borges SV, Ribeiro PC do N, Ruback VR. Aproveitamento alternativo da casca do maracujá-amarelo (Passiflora edulis F. Flavicarpa) para produção de doce em calda. Ciência e Tecnol Aliment. 2002;22:259-62.

59. de Queiroz M do SR, Janebro DI, da Cunha MAL, Medeiros J dos S, SabaaSrur AUO, Diniz M de FFM, et al. Effect of the yellow passion fruit peel flour (Passiflora edulis f. flavicarpa deg.) in insulin sensitivity in type 2 diabetes mellitus patients. Nutr J. England; 2012 Oct;11:89.

60. de Araujo MFM, Veras VS, de Freitas RWJF, de Paula M do L, de Araujo TM, Uchoa LRA, et al. The effect of flour from the rind of the yellow passion fruit on glycemic control of people with diabetes mellitus type 2: a randomized clinical trial. J Diabetes Metab Disord. England; 2017;16:18.

61. WHO | Obesity and overweight. World Health Organization; [cited 2016 Dec 5].

62. van Vliet-Ostaptchouk J V, Snieder H, Lagou V. Gene-Lifestyle Interactions in Obesity. Vol. 1, Current nutrition reports. United States; 2012. p. $184-$ 96. 
63. Backhed F, Ding H, Wang T, Hooper L V, Koh GY, Nagy A, et al. The gut microbiota as an environmental factor that regulates fat storage. Proc Natl Acad Sci U S A. United States; 2004 Nov;101(44):15718-23.

64. Ley RE, Turnbaugh PJ, Klein S, Gordon JI. Microbial ecology: human gut microbes associated with obesity. Nature. England; 2006 Dec;444(7122):1022-3.

65. Ley RE, Backhed F, Turnbaugh P, Lozupone CA, Knight RD, Gordon JI. Obesity alters gut microbial ecology. Proc Natl Acad Sci U S A. 2005;102(31):11070-5.

66. Turnbaugh PJ, Ley RE, Mahowald M a, Magrini V, Mardis ER, Gordon JI. An obesity-associated gut microbiome with increased capacity for energy harvest. Nature. 2006;444(7122):1027-31.

67. Schwiertz A, Taras D, Schafer K, Beijer S, Bos NA, Donus C, et al. Microbiota and SCFA in lean and overweight healthy subjects. Obes (Silver Spring). Nature Publishing Group; 2010;18(1):190-5.

68. Goffredo M, Mass K, Parks EJ, Wagner DA, McClure EA, Graf J, et al. Role of gut microbiota and short chain fatty acids in modulating energy harvest and fat partitioning in youth. J Clin Endocrinol Metab. 2016;101(November):jc.2016-1797.

69. Lyra A, Lahtinen S, Tiihonen K, Ouwehand AC. Intestinal microbiota and overweight. Benef Microbes. Netherlands; 2010 Nov;1(4):407-21.

70. Belzer C, de Vos WM. Microbes inside--from diversity to function: the case of Akkermansia. ISME J. England; 2012 Aug;6(8):1449-58.

71. Santacruz A, Collado MC, Garcia-Valdes L, Segura MT, Martin-Lagos JA, Anjos T, et al. Gut microbiota composition is associated with body weight, weight gain and biochemical parameters in pregnant women. Br J Nutr. England; 2010 Jul;104(1):83-92.

72. Dao MC, Everard A, Aron-Wisnewsky J, Sokolovska N, Prifti E, Verger EO, et al. Akkermansia muciniphila and improved metabolic health during a dietary intervention in obesity: relationship with gut microbiome richness and ecology. Gut. 2016;65(3):426-36.

73. Qin J, Li Y, Cai Z, Li S, Zhu J, Zhang F, et al. A metagenome-wide association study of gut microbiota in type 2 diabetes. Nature. England; 2012 Oct;490(7418):55-60.

74. Everard A, Belzer C, Geurts L, Ouwerkerk JP, Druart C, Bindels LB, et al. Cross-talk between Akkermansia muciniphila and intestinal epithelium controls diet-induced obesity. Proc Natl Acad Sci U S A. 2013;110(22):9066-71.

75. Le Chatelier E, Nielsen T, Qin J, Prifti E, Hildebrand F, Falony G, et al. Richness of human gut microbiome correlates with metabolic markers. Nature. 2013;500(7464):541-6.

76. Sommer F, Stahlman M, Ilkayeva O, Arnemo JM, Kindberg J, Josefsson J, et al. The gut microbiota modulates energy metabolism in the hibernating brown bear Ursus arctos. Cell Rep. United States; 2016 Feb;14(7):165561. 
77. Cummings JH, Englyst HN. Fermentation in the human large intestine and the available substrates. Am J Clin Nutr. 1987;45(5 SUPPL.):1243-55.

78. Roberfroid M, Gibson GR, Hoyles L, McCartney AL, Rastall R, Rowland I, et al. Prebiotic effects: metabolic and health benefits. Br J Nutr. England; 2010 Aug;104 Suppl:S1-63.

79. Chambers ES, Morrison DJ, Frost G. Control of appetite and energy intake by SCFA: what are the potential underlying mechanisms? Proc Nutr Soc. England; 2015 Aug;74(3):328-36.

80. McNeil NI. The contribution of the large intestine to energy supplies in man. Am J Clin Nutr. United States; 1984 Feb;39(2):338-42.

81. Cani PD, Delzenne NM, Amar J, Burcelin R. Role of gut microflora in the development of obesity and insulin resistance following high-fat diet feeding. Pathol Biol (Paris). France; 2008 Jul;56(5):305-9.

82. Nohr MK, Pedersen MH, Gille A, Egerod KL, Engelstoft MS, Husted AS, et al. GPR41/FFAR3 and GPR43/FFAR2 as cosensors for short-chain fatty acids in enteroendocrine cells vs FFAR3 in enteric neurons and FFAR2 in enteric leukocytes. Endocrinology. United States; 2013 Oct;154(10):3552-64.

83. Samuel BS, Shaito A, Motoike T, Rey FE, Backhed F, Manchester JK, et al. Effects of the gut microbiota on host adiposity are modulated by the shortchain fatty-acid binding G protein-coupled receptor, Gpr41. Proc Natl Acad Sci U S A. United States; 2008 Oct;105(43):16767-72.

84. Tolhurst G, Heffron H, Lam YS, Parker HE, Habib AM, Diakogiannaki E, et al. Short-chain fatty acids stimulate glucagon-like peptide-1 secretion via the G-protein-coupled receptor FFAR2. Diabetes. United States; 2012 Feb;61(2):364-71.

85. Minekus M, Marteau P, Havenaar R. Multicompartmental dynamic computer-controlled model simulating the stomach and small intestine. Altern to Lab Anim ATLA. 1995.

86. Minekus M, Smeets-Peeters M, Bernalier A, Marol-Bonnin S, Havenaar R, Marteau $\mathrm{P}$, et al. A computer-controlled system to simulate conditions of the large intestine with peristaltic mixing, water absorption and absorption of fermentation products. Appl Microbiol Biotechnol. 1999;53(1):108-14.

87. Aguirre M, Jonkers DMAE, Troost FJ, Roeselers G, Venema K. In vitro characterization of the impact of different substrates on metabolite production, energy extraction and composition of gut microbiota from lean and obese subjects. PLoS One. United States; 2014;9(11):e113864.

88. Kovatcheva-Datchary P, Egert M, Maathuis A, Rajilić-Stojanović M, De Graaf AA, Smidt H, et al. Linking phylogenetic identities of bacteria to starch fermentation in an in vitro model of the large intestine by RNAbased stable isotope probing. Environ Microbiol. 2009;11(4):914-26.

89. Venema K. Role of gut microbiota in the control of energy and carbohydrate metabolism. Curr Opin Clin Nutr Metab Care. 2010;13(4):432-8. 
Chapter 1 


\section{Chapter 2 \\ Characterization and in vitro digestibility of by-products from Brazilian food industry: cassava bagasse, orange bagasse and passion fruit peel}

Published as:

de Souza CB, Jonathan M, Saad SMI, Schols HA, Venema K. Characterization and in vitro digestibility of by-products from Brazilian food industry: cassava bagasse, orange bagasse and passion fruit peel. Bioactive Carbohydrates and Dietary Fibre. 2018. Oct.(16)90-99. 


\begin{abstract}
The present study characterized selected by-products from Brazilian food industry and their in vitro digestibility. These by-products (cassava and orange bagasses, and passion fruit peels) are potentially rich sources of dietary fibres, but currently they are mostly disposed. Their chemical analyses revealed differences in composition for the same by-product type from different suppliers. Cassava bagasses were mainly composed of starch, with high variability among tested by-products (45\% to $77.5 \%$ starch (w/w)). In vitro experiments using a model of the upper gastrointestinal tract (TIM-1) indicated that cassava bagasses had $\sim 12 \%$ of resistant starch. The main constituents in orange bagasses were free glucose and highly methyl esterified pectin $(\sim 23.5 \%$ of total pectin). Seventy-seven \% of digestible glucose present in the orange bagasse were absorbed within 3 hours during in vitro digestion. Passion fruit peels were a good source of fibres, especially pectin ( $19 \%)$ and (hemi)cellulose $(\sim 16 \%)$. The in vitro experiments indicated that passion fruit peel had slower absorption of glucose than the other by-products, with $80 \%$ of digestible glucose absorbed within 5 hours. In conclusion, the tested by-products are good sources of diverse types of fibres and have a great potential to be incorporated into different food products, thus decreasing food waste and contributing to a sustainable food system.
\end{abstract}




\section{Introduction}

Brazil is the largest producer of orange juice, passion fruit juice and the second largest producer of cassava worldwide (1). A consequence of the technical and economical achievement of the processing industry is that a large amount of underused by-products is formed, which includes peels, seeds, and bagasses. Cassava bagasse is a by-product from the production of cassava starch and cassava flour (Manihot esculenta Crantz) and contains fibre material as well as the residual starch not completely extracted during processing (2). In Brazil, for every ton of cassava used for starch production, around $928 \mathrm{~kg}$ of moist bagasse is generated (2). The orange juice industry produces as by-product orange bagasse that corresponds to around $50 \%$ of the total fruit mass (3). In case of passion fruit, around $40-60 \%$ of the total fruit mass ends up as residue and byproduct consisting of peels and seeds (4).

These by-products can be used for animal feed (low-valued product), but mostly they are simply disposed in the environment without any type of treatment and thus are an environmental hazard (5). Some of the citrus peel is used for pectin production (high-valued product), but the market demand for pectin is low compared to the amount of pectin that could be produced from the worldwide supply of citrus by-product (6). This challenges the food industry to identify ways for a better utilization of these materials aiming for the strengthening of sustainable food use (7). The use of food by-products contributes to a sustainable food system, decreasing the impact that their disposal would have on the environment. Additionally, their utilization is aligned to a responsible use of natural resources that were employed for plants cultivation, such as soil and water, as well as the human labour that was applied (7).

Different by-products, such as chicory root pulp and apple pulp, are rich in fibre, including pectin (8). Currently, dietary fibre is defined as carbohydrate polymers with a degree of polymerization (DP) of three or more, which are neither digested nor absorbed in the human intestine (9). In general, consumption of dietary fibre is associated with intestinal health, reduced cardiovascular disease risks and lower body weights (10). Currently the general consumption of fibre by different populations nonetheless is lower than the recommended amount (10). In Brazil, a survey performed between 2008-2009 revealed that people consumed on average $12.5 \mathrm{~g}$ of fibre/day, much lower than the $30 \mathrm{~g} /$ day recommendation (10).

The high fibre content of by-products such as bagasses and peels makes them a potential dietary fibre food source. The need to increase fibre consumption and the increased concerns about sustainability, are strong stimuli to try to 
incorporate by-products from the food agro-industry into the human diet, e.g. in the form of (new) functional food (components).

Dietary fibre is a term that reflects a heterogeneous group of compounds that differ in their chemical structure and physico-chemical properties (11). Different fibre types may have variable physiological functions or health benefits (12). It is therefore crucial to structurally characterize these by-products before analyzing their potential health benefits (e.g. prebiotic effect). The International Scientific Association for Probiotics and Prebiotics has recently released a new definition for prebiotics: "a substrate that is selectively utilized by host microorganisms conferring a health benefit" (13). Bifidobacterium and Lactobacillus genera are used as biomarkers for intestinal health, since these two genera do not contain any known pathogens and they ferment carbohydrates as preferred carbon- and energy source, producing metabolites known to have positive effects on the host (14). The molecular structure of a prebiotic fibre also determines its physiological effects as well as which microbial species will be able to use it as growth substrate in the intestine (15). We have already demonstrated the potential prebiotic effects of cassava bagasse using an in vitro model that simulates the large intestine (TIM-2) (16). However, it has remained unknown which component was responsible for the observed bifidogenic effects, and whether different batches or origin (and consequently processing) show the same results.

Pectins may be an important component of food by-products. Information about the level of methyl esterification of these by-products is essential for future food application studies, for instance as functional foods aiming for improvement of intestinal health. Animal studies demonstrated that highly methyl esterified (HM) pectins were fermented slower than lowly methyl esterified (LM) pectins $(17,18)$. This is a very important characteristic, since normally most of the carbohydrates are fermented in the proximal colon. Their early depletion may result in fermentation of proteins in the distal colon, with potentially negative effects to the host, such as colon cancer (19). Analysis of the in vitro digestibility of these by-products will indicate to what extent they can reach the colon, to be further fermented by the colonic bacteria.

The aim of the present study was to characterize the composition of selected byproducts from the Brazilian food industry. Their digestibility was investigated in an in vitro model of the upper gastrointestinal tract (TIM-1). Isolation of fibre (alcohol insoluble solids; AIS) from by-products was performed and these samples were also characterized. 


\section{Materials and methods \\ Test products}

Three different cassava bagasses were tested; two of these were from the same supplier but obtained as different batches (Grazimara, Biguaçu, Brazil) - referred to as $\mathrm{CB} 1$ and $\mathrm{CB} 2$, and the third one was from a different company (Lorenz, Indaial, Brazil - referred to as CB3). The bagasse was dried by the suppliers in an oven at $250-300{ }^{\circ} \mathrm{C}$ for $20 \mathrm{~min}$. It was milled using ball-milling (MM2000, Retsch, Haan, Germany).

Two different orange bagasses were tested, both in the form of powder - from Chá\&Cia (Jacareí, Brazil) - denoted as OB1, and from a store with bulk sale in the city centre of Florianópolis (Brazil), referred to as OB2. Three different passion fruit peels were tested, all in the form of powder - from Chá\&Cia, referred to as PFP1, Phytomare (Governador Celso Ramos, Brazil - PFP2), and the last one from the same store as OB2 (Florianópolis, Brazil), denoted as PFP3. According to the manufacturers, only the peels were used to prepare the powder, whereas the seeds had been disposed.

\section{Monosaccharide compositions}

Monosaccharide compositions were determined for test products after acid hydrolysis of all carbohydrates, followed by derivatizing the sugars released into alditol acetates as previously described (20). Briefly, after a pre-hydrolysis step using $72 \%(\mathrm{w} / \mathrm{w})$ sulphuric acid at $30{ }^{\circ} \mathrm{C}$ for 1 hour, samples were hydrolyzed with $1 \mathrm{M}$ sulphuric acid $\left(\mathrm{H}_{2} \mathrm{SO}_{4}\right)$ at $100{ }^{\circ} \mathrm{C}$ for $3 \mathrm{~h}$. Afterwards the monosaccharides released were reduced, acetylated and analyzed with gas chromatography (GC) equipped with a flame ionization detector (FID). Inositol was used as internal standard (21). The total uronic acid content was determined with the automated meta-hydroxydiphenyl assay (22). Galacturonic acid (12.5$100 \mu \mathrm{g} / \mathrm{mL}$ ) was used for calibration.

Pectin levels were calculated from uronic acids (UA), rhamnose (Rha), arabinose (Ara) and galactose (Gal) (w/w \%). Non-starch polysaccharides (NSP) glucose was calculated from the difference between total glucose and starch. Rhamnogalacturonan (RG) backbone was calculated using the levels of Rha (mol $\%$ ), assuming the RG backbone consists of Rha and UA with a ratio of 1:1 (20). The total RG content was calculated as RG backbone plus arabinose and galactose. The homogalacturonan (HG) content was quantified by total UA content minus UA content present in RG backbone (20). 


\section{Quantification of glucose, fructose and sucrose}

High performance anion exchange chromatography (HPAEC) was performed on a ICS5000 system (Dionex Corporation, Sunnyvale, USA) as previously described (20). The flow rate was constant at $0.3 \mathrm{~mL} / \mathrm{min}$. The elution started with $2 \mathrm{~min}$ isocratic elution of $0.1 \mathrm{M}$ sodium hydroxide $(\mathrm{NaOH})$, followed by a $45-\mathrm{min}$ gradient to $400 \mathrm{mM}$ sodium acetate in $0.1 \mathrm{M} \mathrm{NaOH}$. The column was subsequently cleaned for $5 \mathrm{~min}$ in $1 \mathrm{M}$ sodium acetate in $0.1 \mathrm{M} \mathrm{NaOH}$, and reequilibrated under starting conditions for $15 \mathrm{~min}$, before the next sample injection. Test products were suspended in Millipore water $(2 \mathrm{mg} / \mathrm{mL})$ and centrifuged $(10000 \mathrm{xg}, 5 \mathrm{~min})$ before analysis. One part OB supernatant was diluted with four parts of water before analysis. The other samples were diluted with the same volume of water. Glucose, fructose and sucrose at 10, 20 and 30 $\mu \mathrm{g} / \mathrm{mL}$ were used as quantification standards. Chromeleon software version 7 (Dionex) was used for detection and analysis.

\section{Characterization of pectins according to the degree of acetyl- and methyl esterification}

For the quantification of the degree of acetyl- (DA) and methyl (DM) esterification of the uronic acid in the pectins, test products $( \pm 3 \mathrm{mg})$ were saponified in $1 \mathrm{~mL}$ of $\mathrm{NaOH}(0.25 \mathrm{M})$ for $3 \mathrm{~h}$ at room temperature. The amount of methanol released was quantified using a colorimetric method as previously described (23) determined with a spectrophotometer at $420 \mathrm{~nm}$ (Tecan Infinity F500, Männedorf, Switzerland). The amount of acetic acid released during saponification was quantified using the acetic acid assay kit from Megazyme (Bray, Ireland).

Test products were analyzed in triplicate and the average acetic acid or methanol concentration was calculated. The degrees of acetylation and methylation were calculated as moles of acetic acid or methanol per $100 \mathrm{~mol}$ of uronic acid. It is important to mention that apart from DM, some non-pectic cell wall polysaccharides can also contain acetyl groups (20).

\section{Total starch}

Total starch content was analyzed according to the AOAC method (996.11) for samples that may contain D-glucose and/or maltodextrins, using the total starch assay kit from Megazyme. 


\section{Resistant starch}

Resistant starch (RS) was analyzed only in test products with a considerable amount of starch: CB1, CB2, CB3 and PFP3. It was measured in quadruplicate using the resistant starch assay kit (Megazyme).

\section{Alcohol insoluble solids (AIS)}

For all three cassava bagasses (CB1, CB2 and CB3) removal of starch and protein was performed prior to AIS isolation. Test products $(10 \mathrm{~g})$ were suspended in $100 \mathrm{~mL}$ of $0.2 \mathrm{M}$ sodium acetate buffer, heated to $85^{\circ} \mathrm{C}$ and incubated with $75 \mu \mathrm{L}$ thermostable a-amylase $(0.548 \mathrm{U} / \mathrm{g}$, from Bacillus licheniformis, Sigma-Aldrich, Schnelldorf, Germany) for $35 \mathrm{~min}$. After cooling down to $40{ }^{\circ} \mathrm{C}$, additional aamylase $(75 \mu \mathrm{L})$ and $125 \mu \mathrm{L}$ of amyloglucosidase $(11.6 \mathrm{U} / \mathrm{g}$, from Rhizopus, Sigma-Aldrich) were added to the suspensions to hydrolyze any residual starch. The suspensions were incubated at $40{ }^{\circ} \mathrm{C}$ for $3 \mathrm{~h}$ while stirring. Next, they were centrifuged $\left(8000 \mathrm{xg}, 15 \mathrm{~min}, 20^{\circ} \mathrm{C}\right)$. The supernatants were collected and $75 \mathrm{~mL}$ of $0.05 \mathrm{M}$ sodium acetate buffer at pH 5.2, $75 \mu \mathrm{L}$ of a-amylase and $125 \mu \mathrm{L}$ of amyloglucosidase were added to the pellets and incubated overnight at $30{ }^{\circ} \mathrm{C}$ while shaking (supernatants were collected). Samples were centrifuged once more $\left(8000 \mathrm{xg}, 15 \mathrm{~min}, 20^{\circ} \mathrm{C}\right.$ ) and $75 \mathrm{~mL}$ of $0.05 \mathrm{M}$ sodium acetate buffer at $\mathrm{pH}$ 5.2 and $125 \mu \mathrm{L}$ of amyloglucosidase were added to the pellets. Thereafter samples were incubated at $40{ }^{\circ} \mathrm{C}$ for $1 \mathrm{~h}$ while stirring. At this point, the supernatants collected were mixed again with the pellets, $\mathrm{pH}$ was adjusted to 6.0 and $125 \mu \mathrm{L}$ of protease (Subtilisin A from B. licheniformis (Megazyme)) was added.

Samples were incubated at $50^{\circ} \mathrm{C}$ for $1 \mathrm{~h}$ while stirring. Ethanol was added to $70 \%$ $(\mathrm{v} / \mathrm{v})$ and the process continued as described below.

Samples of orange bagasses (OB1 and OB2) and passion fruit peels (PFP1 and PFP2) were directly used for AIS isolation (without prior removal of starch and protein). Test products were suspended in $80 \%(\mathrm{v} / \mathrm{v}$ ) aqueous ethanol and incubated at $4{ }^{\circ} \mathrm{C}$ for $1 \mathrm{~h}$ while stirring. The suspensions were centrifuged (8000 $x \mathrm{~g}, 20^{\circ} \mathrm{C}, 15 \mathrm{~min}$ ) and the supernatants were discarded. The pellets was washed with $80 \%(\mathrm{v} / \mathrm{v})$ ethanol and centrifuged and this step was repeated until the extracts had no detectable carbohydrates, checked with the phenol sulfuric acid colour assay (24). The final residues were suspended in water, freeze dried and denoted as alcohol insoluble solids (AIS).

\section{Total protein}

Total protein was estimated from the total nitrogen analyzed using the "Dumas" method on a Flash EA $1112 \mathrm{~N}$ analyser (Thermo Fisher Scientific, Waltham, MA, 
USA) (25). D-Methionine (Acros Organics, NJ, USA) was used for calibration and cellulose (Fluka, Buchs, Switzerland) served as a blank. Protein content was calculated as nitrogen content x 6.25 .

\section{In vitro digestibility}

The by-products $\mathrm{CB} 1, \mathrm{CB} 2, \mathrm{CB} 3, \mathrm{OB} 2$ and PFP3 were selected for in vitro digestion studies, using the TNO intestinal tract model (TIM-1) (Supplemental Figure 1). TIM-1 is a dynamic in vitro model that simulates the stomach and small intestine. It consists of four compartments that simulate the stomach, duodenum, jejunum and ileum respectively, which are connected by computer controlled valves (26). The temperature was kept at $37{ }^{\circ} \mathrm{C}$ (body temperature). The secretion of fluids and enzymes in each compartment was set to mimic the physiological conditions of healthy human adults. Peristaltic movements are mimicked through the contraction of the flexible walls, which are also responsible for controlling gastric emptying and food transit (26).

The contents of the jejunum and ileum compartments were dialyzed continuously using hollow fibre semi-permeable membranes (Sureflux 07L, Nipro Europe, Zaventem, Belgium - cut-off 5-10 kDa), resembling the absorption of the digested products such as monosaccharides, oligosaccharides, amino acids and small peptides by intestinal cells (26).

\section{Set up of the TIM-1 experiments}

All digestive fluids were freshly prepared prior to the performance of each TIM1 experiment as previously described (27).

The amount of CB2, CB3, OB2 and PFP3 was set at 7.5 grams, and for CB1, 15 grams, reflecting their different capacity to bind (large amounts of) water. Water binding would increase their volume and viscosity, which could surpass the capacity of the system. To avoid this inconvenience, the quantities were adjusted as indicated.

Samples were tested without a meal matrix, but their transit through the system was mimicked as if they were part of a meal.

Tested by-products were mixed with water and gradually added to the salivary/gastric juice at room temperature and the $\mathrm{pH}$ was set at 5.2 with hydrochloric acid $(\mathrm{HCl})$ or $\mathrm{NaOH}$. The total volume added to the stomach was $300 \mathrm{ml}$ (referred to as meal).

\section{Simulated gastrointestinal conditions}

Artificial oral fluid with $\alpha$-amylase (A6380-1G, Sigma-Aldrich, St. Louis, USA), gastric juice with gastric lipase (F-AP 15, Amano, Nagoya, Japan) and pepsin 
(Sigma-Aldrich, St. Louis, USA) were gradually added into the gastric compartment (28). Pancreatin (Pancrex-Vet, Pfizer, Karlsruhe, Germany) was prepared by centrifugation after stirring $17.5 \mathrm{~g}$ powder in $250 \mathrm{ml}$ water.

Pancreatin, bile solution ( $0.8 \%$, porcine bile - Sigma-Aldrich, St. Louis, USA) and electrolytes were gradually added to the duodenal compartment during the experimental run (28). The composition and secretion of fluids simulated the physiological levels at fed state. Details have been published before (28). Digestion and absorption was simulated during a $6 \mathrm{~h}$ experimental run, after which approximately $95 \%$ of the meal passed through the upper gastrointestinal tract (27).

\section{Sampling}

Samples from the gastric intake (meal) were taken just before its addition to the stomach compartment.

Ileal efflux (indigestible portion) was kept on ice to stop the digestive activity. During the experiments, samples from the ileal efflux were taken every hour. Dialysate samples from the jejunum and ileum compartments (absorbed portions) were also collected every hour. After $6 \mathrm{~h}$, indigestible material (residues remaining in the stomach, duodenum, jejunum and ileum) was taken, in order to make a mass-balance. For all sampling times, $\mathrm{pH}$ and total volume were measured and recorded. Samples were stored at $-18{ }^{\circ} \mathrm{C}$ until analyses.

\section{Analyses}

Meal, ileal efflux and dialysate samples (jejunum and ileum compartments) were analyzed for total nitrogen using the Kjeldahl method, and glucose using the acid hydrolysis method on a Cobas Mira plus autoanalyser (Roche) by Bio-aNAlytiX (Mook, the Netherlands).

\section{Results and discussion}

\section{Cassava bagasses}

Total sugar composition of CB1 and CB2 was very similar, mainly consisting of glucose $-77.7-79.3 \%(\mathrm{w} / \mathrm{w})$ (Table 1$)$. This glucose originated largely from starch because the analysis of CB1 and CB2 revealed the presence of $77.4 \%$ and $70.5 \%$ starch, respectively. They had low amounts of small sugars (glucose, fructose and sucrose), 1.42 and $1.25 \%(\mathrm{w} / \mathrm{w}$ ) in total for CB1 and CB2, respectively (Table 1). The amount of resistant starch (RS) was negligible $(<1 \%)$. Glucose levels from non-starch polysaccharides (NSP - cellulose and hemicellulose) were different for these batches of cassava bagasse. CB1 had $0.3 \%$ of NSP glucose, while CB2 had a higher amount, $8.8 \%$ of NSP glucose (Table 1 ). 


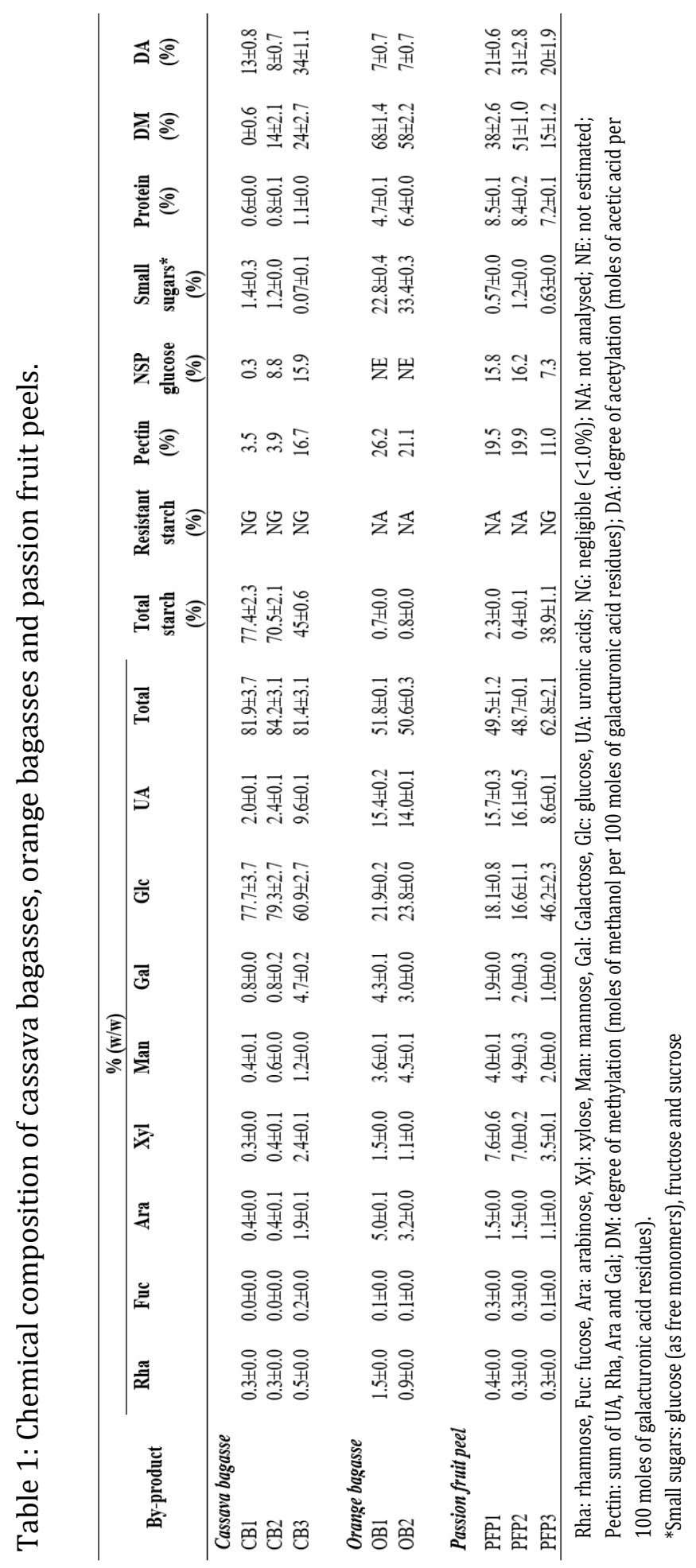


CB3 showed a different composition. This bagasse had lower amounts of glucose, part of which from starch $(45 \%(\mathrm{w} / \mathrm{w}))$, but also derived from cellulose and hemicellulose (15.9\% of NSP glucose). RS was also negligible, as were small sugars $(<1 \%)$.

The amounts of carbohydrates and starch found in CB1 and CB2 were higher than in CB3 and compared to values reported in other studies, which vary from around 40 to $64 \%$ for carbohydrates (29) and $47 \%$ for starch (30). These differences could be due to the manufacturing process of peeling cassava and producing cassava flour. CB1 and CB2 were generated by a small family company, where the farmers were responsible for planting, harvesting and peeling the cassava, and afterwards producing the flour. On the other hand, CB3 originated from a large-scale industrial processing of cassava. Therefore, the industrial process was more efficient in peeling and removing the starch from the bagasse than the artisanal process, explaining the lower amount of starch and the higher amount of NSP in CB3. The handmade processing that CB1 and CB2 went through can also explain the differences in starch and NSP glucose amounts found in these two batches, since the artisanal process of peeling cassava is more prone to variation.

CB3 had a higher amount of pectin $(16.7 \%(\mathrm{w} / \mathrm{w}))$ compared with CB1 and CB2. CB1 and CB2 had the lowest DM for all tested compounds - 0 and $14 \%$, respectively (Table 1), probably due to low NSP and UA levels, which may impair the accurate DM analysis. DM of CB3 was $24 \%$ and DA $34 \%$.

Analysis of protein in the CB samples demonstrated low amounts of this macronutrient, varying from 0.6 to $1.1 \%$ (Table 1 ), in accordance with findings in the literature, where protein levels range from 0.32 to $1.9 \%$ (29).

Carbohydrate and protein contents determined accounted for $\sim 83 \%$ for CB1, $\mathrm{CB} 2$ and $\mathrm{CB} 3$ of the total mass. Remaining moisture, ash and lipids represent the residual percentages (29).

AIS obtained after starch and protein removal was composed of 57.1, 57.8 and $61.6 \%(\mathrm{w} / \mathrm{w})$ carbohydrates for CB1, CB2 and CB3, respectively (Table 2). The enzymatic treatment of starch removal was not completely efficient, since small amounts of starch were still present in CB1 and CB2 (3.0 and 3.4\%, respectively). The same occurred for protein, where AIS of cassava bagasses had even higher amounts of protein compared to CB prior to AIS isolation (Table 2). Proteins can be from enzymes added during the sample treatment. AIS characterization revealed that CB1, CB2 and CB3 were composed for $27-28 \%$ of pectins which were lowly methylesterified $(<30 \%)$ (Table 2 ). 


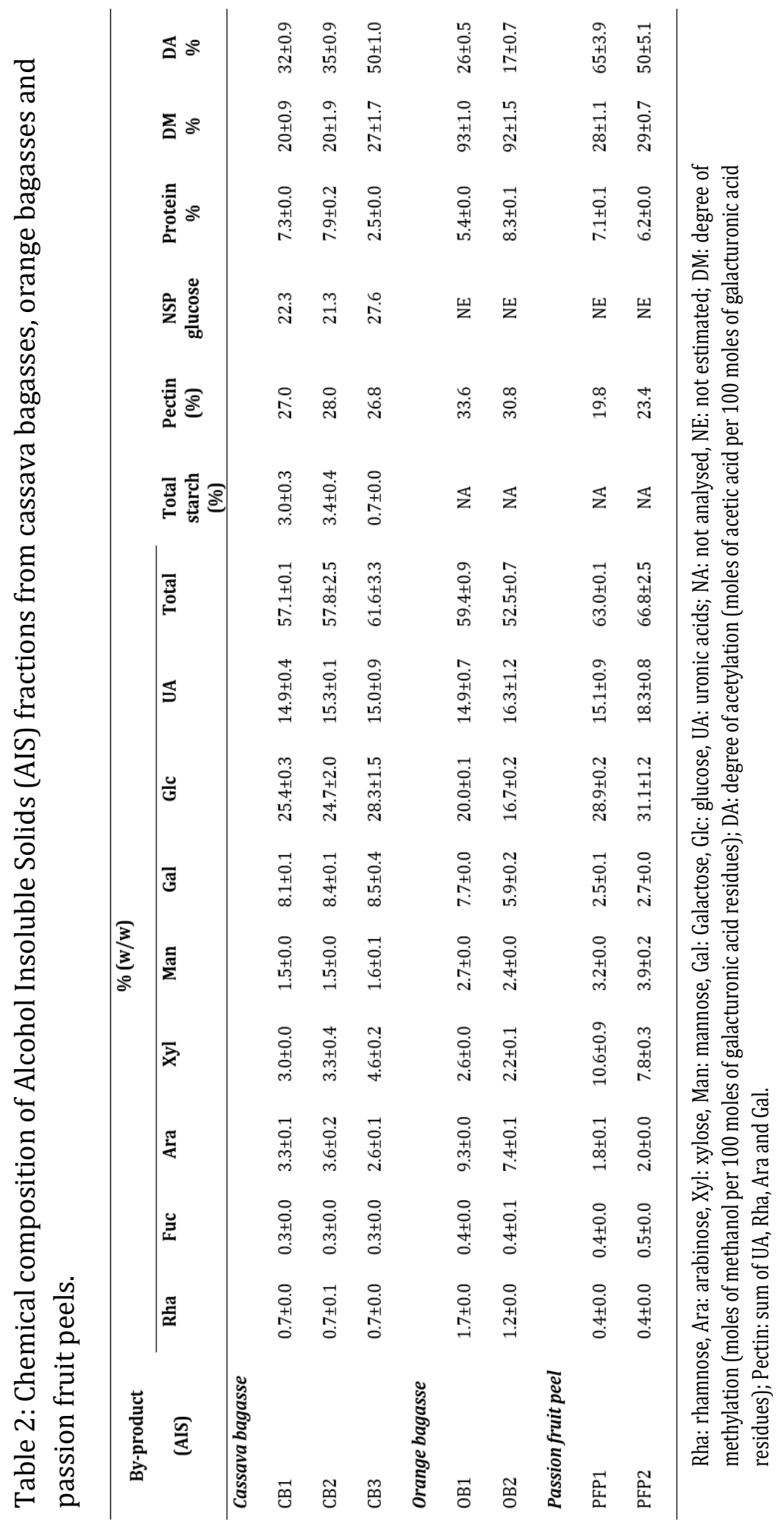


For all three cassava bagasses, pectin in the AIS fractions were predominantly present as homogalacturonan $(\mathrm{HG})$ segments, concluded from the ratio UA:Rha (data not shown) 24:1 (CB1 and CB2) and 22:1 (CB3). The successful isolation of fibre (AIS) from all three tested cassava bagasses indicated that this may represent an alternative procedure for their further utilization. AIS characterization revealed a significant higher concentration of pectin and (hemi)cellulose (Table 2) when compared to their raw by-products (Table 1).

In vitro digestibility experiments of cassava bagasses demonstrated that around $87 \%$ of the glucose present was digestible (Figure 1.1-1.3-D). It should be noted that the analysis used for glucose measurement from TIM-1 samples encompasses free glucose and starch-derived glucose only. Previously we hypothesized that a large part of the remaining starch present in all cassava bagasses was resistant starch type 1 (16) - physically inaccessible starch (31). This was not confirmed here: the digestibility experiments showed that on average $12 \%$ of glucose from the three tested cassava bagasses were not digestible. Although the chemical analysis of cassava bagasses for RS showed negligible amounts (Table 1), it is important to highlight that the in vitro digestibility experiment mimics the time of food to transit through the upper gastrointestinal tract, and the definition of RS is "the starch that is not absorbed in the small intestine of humans" (31). The indigestible portion by definition thus represents RS. Despite CB3 had higher amounts of NSP glucose and pectin (which could decrease transit time of the digest and glucose absorption $(32,33)$ ), all three cassava bagasses showed a similar glucose digestibility rate, with a peak of absorption occurring during the second hour of digestion in the jejunum segment, as can be seen in Figure 1.1-1.3-A. Cumulative absorption of glucose from the three cassavas showed that after $3 \mathrm{~h}$ mostly if not all digestible glucose had been absorbed (Figure 1.1-C). Protein absorption of the three cassava bagasses tested occurred fast, mostly during the first $2 \mathrm{~h}$ (Figure 1.1-1.3-B), likely due to their low protein content (0.64-1.07\%) (Table 1).

A study comparing 15 samples of cassava flour from different regions in Brazil showed that the amount of starch varied between 81.9-92.1 g/100 g, and fibre varied between 0.5-2.7 g/100 g (34). CB1 had similar composition as cassava flour. Nevertheless, CB2 had lower amounts of starch and higher quantities of fibres than cassava flour. These by-products thus may be suitable as a substitute for food products that are normally prepared with cassava flour, such as cakes, cookies, breads and typical Brazilian dishes like "farofa", although systematic analyses of sensorial features and consumer acceptance are required. 

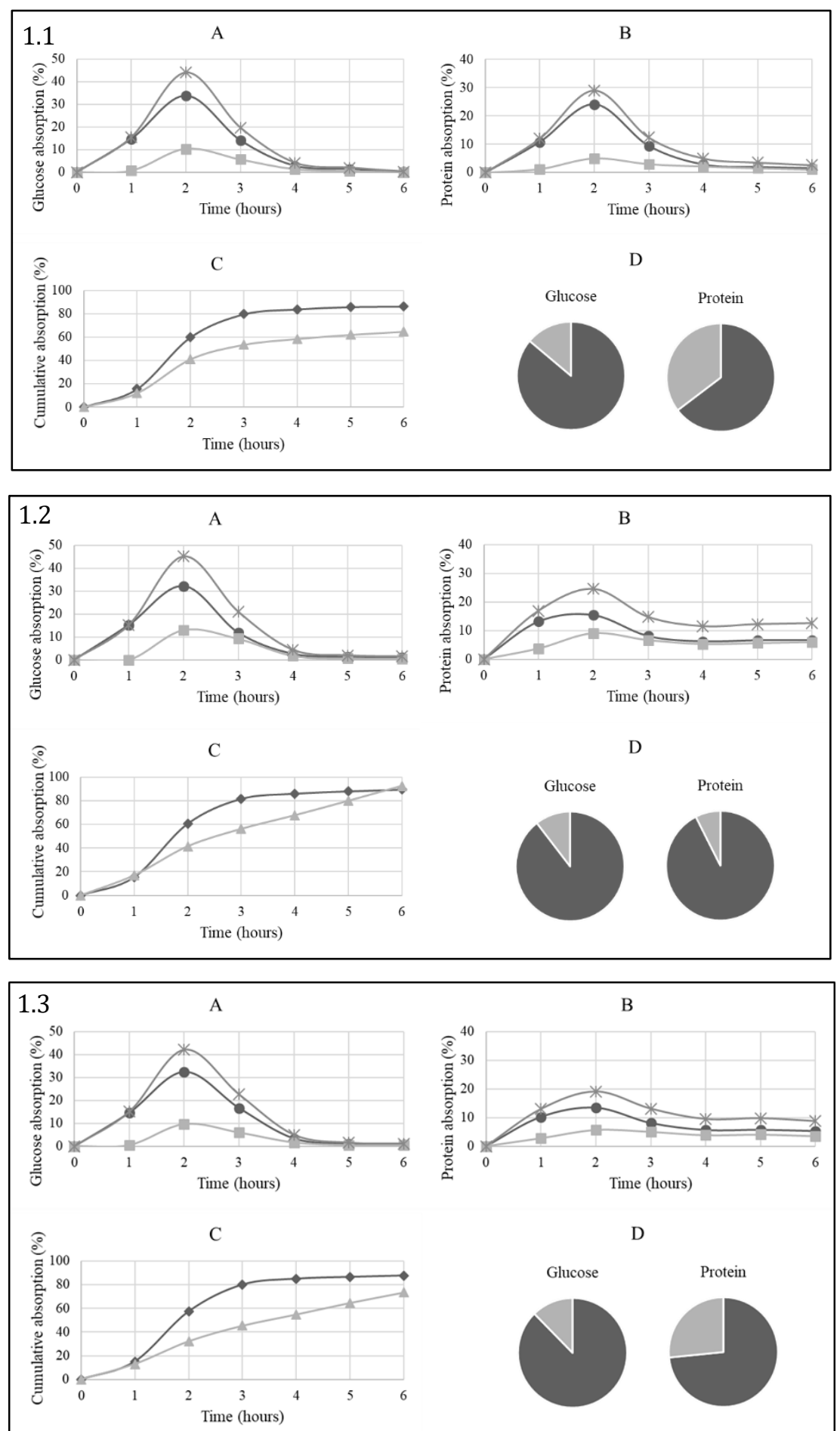

D

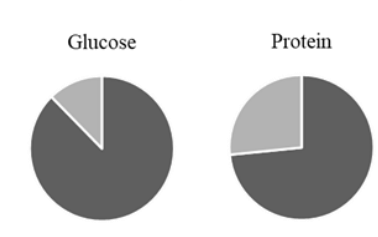

$\longrightarrow$ Absorption jejunum $\longrightarrow$ Absorption ileum $\longrightarrow$ Total absorption

$\underset{\sim}{\longrightarrow}$ Grotein

Digested Indigested 
Figure 1.1-1.5: A: Rate of glucose absorption (\%) on TIM-1 during 6 h experimental run. B: Rate of protein absorption (\%) on TIM-1 during $6 \mathrm{~h}$ experimental run. C: Cumulative absorption (\%) of glucose and protein during $6 \mathrm{~h}$ experimental run. D: Final percentage $(\%)$ of digested and indigested glucose and protein after $6 \mathrm{~h}$ experimental run on TIM-1. 1.1-CB1; 1.2-CB2; 1.3-CB3.

Importantly, cassava and its derived products (cassava flour and tapioca starch) constitute an important source of energy, especially for the population from various remote regions in Brazil because this crop is resistant to poor nutrient and dry soil (2). Discarding such by-products therefore is a waste of energy and fibre source for humans.

CB3 is richer in pectin, cellulose and hemicellulose than the other two cassava bagasses tested. Pectin is known for its effects on satiety and decreasing cholesterol and glucose absorption (33) and its fermentation by gut microbiota. Cellulose and hemicellulose are mainly known for their benefits in increasing volume and frequency of defecations (32), thereby contributing to alleviating constipation. This by-product could be incorporated into different food products, such as fibre supplement in whole-grain breads and cookies.

\section{Orange bagasses}

Samples of orange bagasse from the two different suppliers tested showed a very similar composition (Table 1). The total amount of carbohydrates accounted for $50.6-51.8 \%(w / w)$. Considerable percentages of small sugars (like glucose, fructose and sucrose) were present - 22.8 and $33.4 \%(\mathrm{w} / \mathrm{w})$ for OB1 and OB2, respectively (Table 1), indicating the presence of some residual juice in these byproducts. The high amounts of fructose and sucrose present in these samples did not allow an accurate estimation of the amount of NSP glucose (cellulose and hemicellulose) because fructose is not stable during acid hydrolysis (35) and may partially be converted into glucose and mannose.

OB1 and OB2 showed small differences in protein levels: $4.7 \%$ and $6.4 \%$, respectively (Table 1). Similarly, Lopes et al., (36) reported an amount of $6.5 \%$ of protein in orange bagasse. These two by-products contained only minor amounts of starch, resistant starch analysis therefore was not performed.

Carbohydrate and protein contents accounted for $\sim 57 \%$ of the total mass for OB1 and OB2. The remaining material was not identified and may be composed of moisture, ash, lipids, lignin, and part of the fructose that was degraded during acid hydrolysis, and organic acids, as reported elsewhere (36,37).

Characterization of the AIS fraction from orange bagasses showed that OB1 and OB2 were composed for $33.6 \%$ and $30.8 \%$ of pectin, respectively (Table 2). These pectins were highly methylesterified ( $93 \%$ and $92 \%$, respectively). The 
ratio of Ara:Gal 19:13 for OB1 and 17:11 for OB2 indicates that the RG regions are slightly dominated by arabinose. They had relatively high amounts of arabinose (OB1 9.3\% and OB2 7.4\% (w/w)), possibly originating from the arabinan side chains of pectin (Table 2). The Rha:UA ratios of 3:22 (OB1) and 3:28 (OB2) indicate that these AIS fractions were rich in HG.

In vitro experiments revealed that for $\mathrm{OB} 2,86.3 \%$ of glucose present in the form of small sugars and starch was digestible (Figure 1.4-D), with $\sim 77 \%$ absorbed within $3 \mathrm{~h}$ (Figure 1.4-C). It thus appears reasonable to conclude that most of the digestible glucose from OB2 originated from free molecules of glucose and sucrose, since the amount of starch was quite low (Table 1). Digestion and absorption of protein from OB2 occurred mainly in the first $2 \mathrm{~h}$ of the experiments, in the jejunum compartment (Figure 1.4-C).

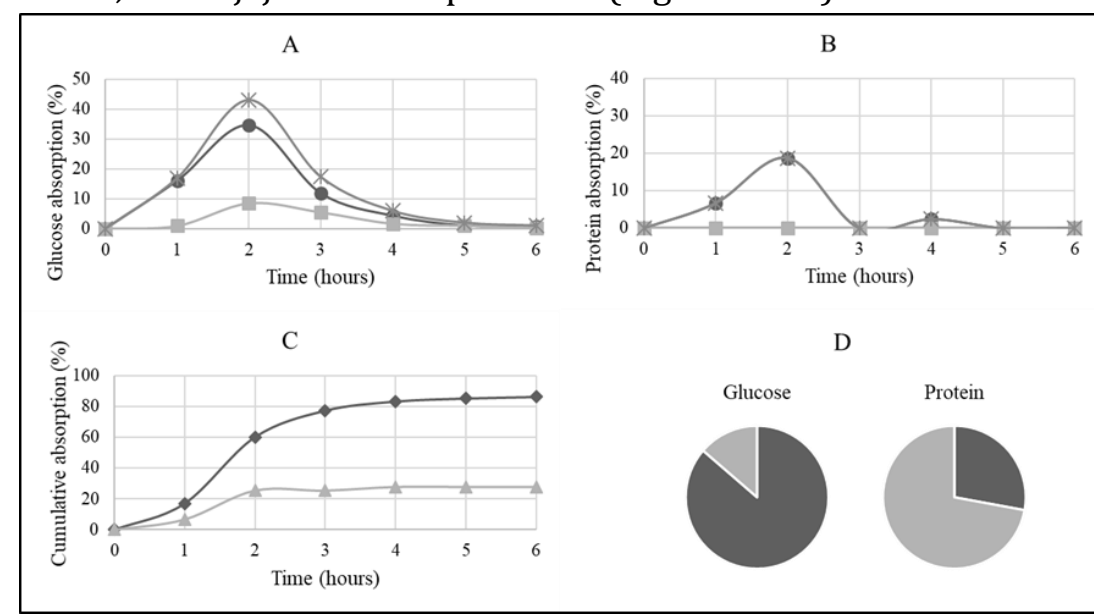

$\longrightarrow$ Absorption jejunum $\longrightarrow$ Absorption ileum $\longrightarrow$ Total absorption

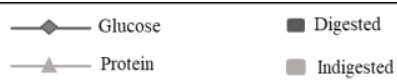

Figure 1.4: OB2

Characterization and in vitro digestibility of orange bagasses revealed that they could be used as a fibre supplement. Pectins are known for their capacity to decrease lipids and glucose absorption in the small intestine, thereby lowering blood lipids and attenuating blood glucose response (10).

\section{Passion fruit peels}

Data obtained from sugar composition analysis of the three tested passion fruit peels shows that PFP1 and PFP2 have a similar composition (Table 1). PFP1, PFP2 and PFP3 had in total 49.5, 48.7 and $62.8 \%(\mathrm{w} / \mathrm{w})$ of carbohydrates. The amounts of small sugars in PFP1 and PFP3 were lower than 1\% (w/w), and 1.2\% for PFP2 (Table 1). PFP1 and PFP2 had higher amounts of pectin $(\sim 20 \%)$ and NSP glucose $(\sim 16 \%)$ than PFP3 $(11.0 \%$ of pectin and $7.3 \%$ of NSP glucose) 
(Table 1). Previous analysis of yellow passion fruit peel revealed an amount of $16.4 \%$ pectin in samples without any type of treatment (only washed and dried peels), and $13.5 \%$ of pectin in samples that passed through an additional treatment step (soaking in water for $12 \mathrm{~h}$ ) (38). Different ways of processing passion fruit by-products thus influence their chemical properties and may explain the differences found, although the exact method of PFP processing for our samples is not known.

The PFP1 and PFP2 AIS fractions were composed of 63 and $66.8 \%$ carbohydrates (Table 2). From these, $19.8 \%$ and $23.4 \%$ were represented by pectins (PFP1 and PFP2, respectively), which were lowly methylesterified (PFP1 28\% and PFP2 $29 \%$ ). Coelho et al. (2017) reported that non-treated passion fruit peels were lowly methylesterified. Lowly methylesterified pectins can be used in food products with the aim of increasing their viscosity without the need to add sugar, as is the case of highly methylesterified pectins (38). The Rha:UA ratios of PFP1 (1:21) and PFP2 (1:25) indicate that pectin was composed predominately of HG. For PFP1, RG regions were composed almost equally of arabinose and galactose (Ara:Gal - 3:4). The ratio of Ara+Gal/Rha indicates that PFP2 had similar arabinose/galactose side chains as PFP1 (7 and 8 for PFP1 and PFP2, respectively).

The amount of starch present in PFP3 (38.9\%) was much higher than in the other two samples analyzed $(<3 \%$; Table 1$)$. This was an unexpected high amount of starch. PFP3 most likely is not a pure passion fruit peel product, and may be contaminated with other fruit by-products/starch. For this reason, AIS isolation and characterization was not performed for PFP3.

Passion fruit peel carried the highest percentage of protein compared with the other by-products analyzed here. The amounts for PFP1, PFP2 and PFP3 were 8.5, 8.4 and 7.2\%, respectively. Cazarin et al (2014) found a lower value in their analysis, $3.94 \%$, whereas Coelho and collaborators (38) reported a value of $3.8 \%$ in their samples without treatment, and $2.5 \%$ in samples that passed through a treatment (soaking in water for $12 \mathrm{~h}$ ).

In vitro experiments with PFP3 indicated that $83 \%$ of the glucose was digestible. As already explained, this by-product batch showed an unusual amount of starch, which was considered by us as a "contamination" with another byproduct/starch. Thus, here the amount of digestible glucose can be either from free molecules of glucose or sucrose (although they were present in very low amounts, $0.63 \%(\mathrm{w} / \mathrm{w}$ ) in total), as well as from the "unusual" starch present. Cumulative absorption of glucose from PFP3 showed a slightly slower absorption when compared to the other by-products tested - with $80 \%$ of the digestible glucose being absorbed within $5 \mathrm{~h}$ (Figure 1.5-C). Our in vitro 
experiments showed that protein from PFP3 was mostly indigestible (95.4\%) (Figure 1.5-D). It is likely that the indigestible protein originated from the digestive juices that had been added to the system (such as pancreatin and bile).
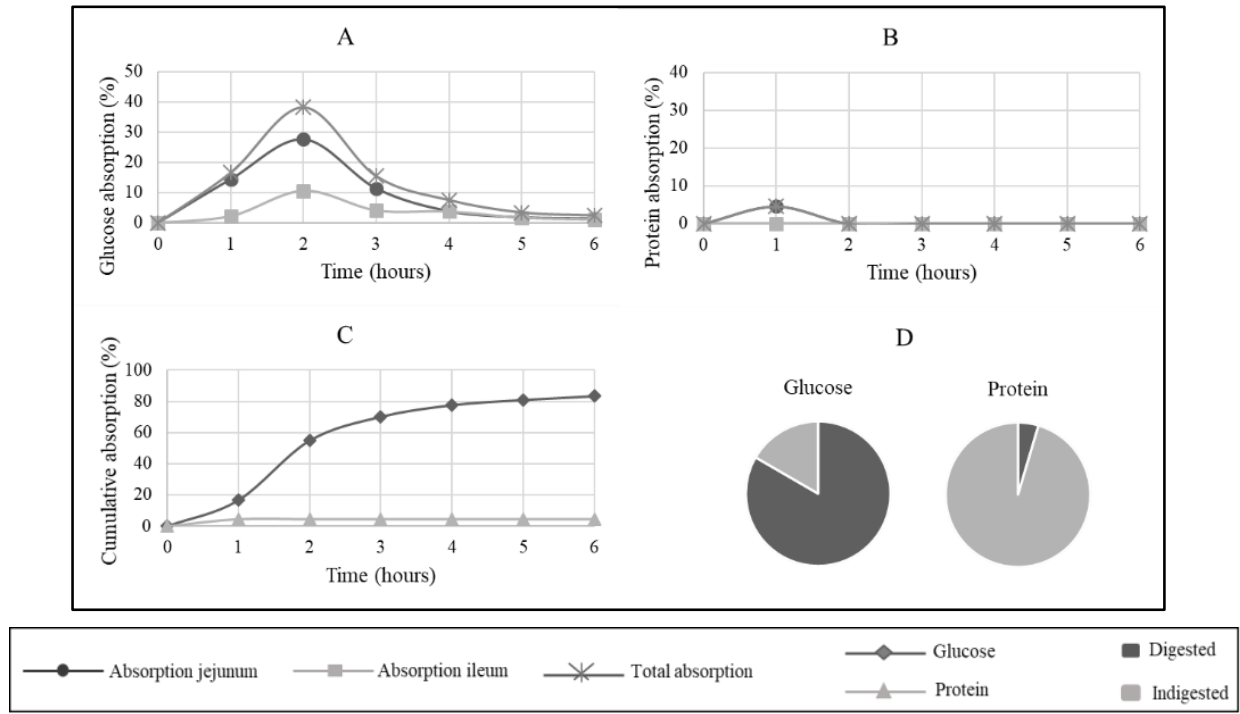

Figure 1.5: PFP3

Characterization of passion fruit peels demonstrated that they are a good source of fibres, being an alternative for fibre supplement that could be used either directly by consumers in different food preparations or by the food industry with the objective to use a natural and sustainable ingredient. However, it is important to be aware of the origin of these by-products, since here we showed that one of the samples was contaminated with a significant amount of starch.

\section{Conclusions}

The detailed analyses performed indicated that different samples of the same type of by-product varied in some aspects, such as pectin levels and composition, and starch amounts. These differences in composition are likely to be due to diversity in the origin and state of development of the plant at harvesting, that in turn may give a distinct chemical and nutritional composition (40), as well as related to the manufacturing process of the products.

Characterization of cassava bagasses revealed that most of their remaining starch was digestible. In this case, cassava bagasse can be used as a source of energy and may be applicable as a substitute to cassava flour, thus reducing the amount of waste material. This could have a positive impact mainly for small farmers/producers, which would increase their income or improve their nutritional status. Fibre isolation (AIS) might be an alternative procedure for 
further utilization of cassava bagasse as a food product/ingredient source of fibre. AIS characterization showed a significant higher concentration of pectin and (hemi)cellulose than the raw by-product.

Orange bagasse analyses demonstrated that both samples were very similar in their chemical structure. They were rich in small sugars and pectin. Pectin characterization showed that HG was predominant in their structure, and they were highly methylesterified.

Characterization of passion fruit peels showed that they are good sources of fibres, especially pectin and (hemi)cellulose.

Regarding pectins, our results suggest that pectins from cassava bagasse, orange bagasse and passion fruit peel are diverse not only in their amount, but also in their chemical structure. Here we have demonstrated that the degree of methylation and acetylation vary according to the by-product, as well as the proportion of $\mathrm{HG}$ and $\mathrm{RG}$ in their structure.

To conclude, we have studied the chemical characteristics and digestibility of selected by-products from the Brazilian food industry, showing that they provide interesting materials for incorporation into different food products, decreasing food waste and contributing to a sustainable food system. These fibre fractions (AIS), either isolated or within the original by-products, are most likely fermented by the gut microbiota, which is the topic of future experiments.

\section{Conflict of interest}

The authors declare that there are no conflicts of interest.

\section{Acknowledgements}

We acknowledge Christiane Rösch from Wageningen University for her analytical support with the sugar composition experiments and analysis, Mark Jelier from Triskelion for his analytical support in handling TIM-1. We also thank the Brazilian companies Chá\&Cia, Lorenz, Phytomare and Grazimara that kindly provided by-product samples for these experiments. We kindly acknowledge Geraldo Bussolo and Zélia Maria Bussolo for collecting and sending samples of orange bagasse and passion fruit peel from Brazil (Florianópolis). We thank Prof. Lubbert Dijkhuizen from Groningen University for critically reviewing our manuscript.

\section{Financial support}

Carlota Bussolo de Souza received a scholarship from CNPq (National Council for Scientific and Technological Development) - Brazil - under the Program "Science 
Chapter 2

without Borders" (grant number 246027/2012-6). Maastricht University campus Venlo financed the experiments and analyses. 


\section{References}

1. Ministério da Agricultura. Citrus [Internet]. Vol. 2014. 2014 [cited 2016 Jan 20]. Available from:

http://www.agricultura.gov.br/vegetal/culturas/citrus

2. Fiorda FA, Soares Júnior MS, da Silva FA, Fontinelle Souto LR, Eiras Grossmann MV. Farinha de bagaço de mandioca: Aproveitamento de subproduto e comparação com fécula de mandioca. Pesqui Agropecu Trop. 2013;43(4):408-16.

3. Romero-Lopez MR, Osorio-Diaz P, Bello-Perez LA, Tovar J, BernardinoNicanor A. Fiber concentrate from orange (Citrus sinensis L.) bagase: characterization and application as bakery product ingredient. Int J Mol Sci. 2011;12(4):2174-86.

4. de Oliveira LF, Nascimento MRF, Borges SV, Ribeiro, do Nascimento PC, Ruback VR. Aproveitamento alternativo da casca do maracujá-amarelo. Ciência e Tecnol Aliment. 2002;22(3):259-62.

5. Helkar P, Sahoo A, Patil N. Review: food industry by-products used as a functional food ingredients. Int J Waste Resour. 2016;6(3):1-6.

6. Manderson K, Pinart M, Tuohy KM, Grace WE, Hotchkiss AT, Widmer W, et al. In vitro determination of prebiotic properties of oligosaccharides derived from an orange juice manufacturing by-product stream. Appl Environ Microbiol. 2005;71(12):8383-9.

7. Meybeck A, Gitz V. Sustainable diets within sustainable food systems. Proc Nutr Soc. 2017 Feb;76(1):1-11.

8. Ramasamy US. The role of soluble and insoluble fibers during fermentation of Chicory root pulp. Doctoral dissertation. Wageningen University; 2014.

9. Stephen AM, Champ MM-J, Cloran SJ, Fleith M, van Lieshout L, Mejborn H, et al. Dietary fibre in Europe: current state of knowledge on definitions, sources, recommendations, intakes and relationships to health. Nutr Res Rev. 2017 Jul 5;1-42.

10. Latulippe ME, Meheust A, Augustin L, Benton D, Bercik P, Birkett A, et al. ILSI Brazil International Workshop on Functional Foods: a narrative review of the scientific evidence in the area of carbohydrates, microbiome, and health. Food Nutr Res. 2013;57(10):7.

11. Jonathan MC. Monitoring the degradation of individual dietary fibres in pig models. Doctoral dissertation. Wageningen University; 2013.

12. Jones JM. CODEX-aligned dietary fiber definitions help to bridge the "fiber gap." Nutr J. 2014;13(34):1475-2891.

13. Gibson GR, Hutkins R, Sanders ME, Prescott SL, Reimer RA, Salminen SJ, et al. Expert consensus document: The International Scientific Association for Probiotics and Prebiotics (ISAPP) consensus statement on the definition and scope of prebiotics. Nat Rev Gastroenterol Hepatol. 2017 Aug;14(8):491-502.

14. Macfarlane S, Macfarlane GT, Cummings JH. Review article: Prebiotics in the gastrointestinal tract. Aliment Pharmacol Ther. 2006;24(5):701-14. 
15. Martinez RC, Bedani R, Saad SM. Scientific evidence for health effects attributed to the consumption of probiotics and prebiotics: an update for current perspectives and future challenges. $\mathrm{Br} \mathrm{J}$ Nutr. 2015;114(12):1993-2015.

16. Bussolo de Souza C, Roeselers G, Troost F, Jonkers D, Koenen ME, Venema K. Prebiotic effects of cassava bagasse in TNO's in vitro model of the colon in lean versus obese microbiota. J Funct Foods. 2014;11(C):210-20.

17. Tian L, Scholte J, Borewicz K, van den Bogert B, Smidt H, Scheurink AJW, et al. Effects of pectin supplementation on the fermentation patterns of different structural carbohydrates in rats. Mol Nutr Food Res. 2016 Oct;60(10):2256-66.

18. Dongowski G, Lorenz A, Proll J. The degree of methylation influences the degradation of pectin in the intestinal tract of rats and in vitro. J Nutr. 2002 Jul;132(7):1935-44.

19. den Besten G, van Eunen K, Groen AK, Venema K, Reijngoud DJ, Bakker $\mathrm{BM}$. The role of short-chain fatty acids in the interplay between diet, gut microbiota, and host energy metabolism. J Lipid Res. 2013;54(9):232540.

20. Ramasamy US, Gruppen H, Schols HA. Structural and water-holding characteristics of untreated and ensiled chicory root pulp. J Agric Food Chem. 2013 Jun;61(25):6077-85.

21. Englyst HN, Cummings JH. Simplified method for the measurement of total non-starch polysaccharides by gas-liquid chromatography of constituent sugars as alditol acetates. Analyst [Internet]. 1984;109(7):937-42.

22. Thibault JF. Automated-method for the determination of pectic substances. Leb Technol. 1979;12(5):247-51.

23. Guillotin SE, Van Loey A, Boulenguer P, Schols HA, Voragen AGJ. Rapid HPLC method to screen pectins for heterogeneity in methyl-esterification and amidation. Food Hydrocoll. 2007;21(1):85-91.

24. Dubois M, Gilles KA, Hamilton JK, Rebers Pa, Smith F. Colorimetric method for determination of sugars and related substances. Anal Chem. 1956;28(3):350-6.

25. Pustjens AM, De Vries S, Gerrits WJ, Kabel MA, Schols HA, Gruppen H. Residual carbohydrates from in vitro digested processed rapeseed (Brassica napus) meal. J Agric Food Chem. 2012/07/18. 2012;60(34):8257-63.

26. Minekus M, Marteau P, Havenaar R. Multicompartmental dynamic computer-controlled model simulating the stomach and small intestine. Altern to Lab Anim ATLA. 1995;23:197-209.

27. Schnorr SL, Crittenden AN, Venema K, Marlowe FW, Henry AG. Assessing digestibility of Hadza tubers using a dynamic in-vitro model. Am J Phys Anthropol. 2015 Nov;158(3):371-85.

28. Havenaar R, de Jong A, Koenen ME, van Bilsen J, Janssen AM, Labij E, et al. Digestibility of transglutaminase cross-linked caseinate versus native caseinate in an in vitro multicompartmental model simulating young child 
and adult gastrointestinal conditions. J Agric Food Chem. 2013;61(31):7636-44.

29. Jasko AC, Andrade J de, Padilha L, Pauli RB de, Quast LB, Schnitzler E, et al. Caracterização físico-química de bagaço de mandioca in natura e após tratamento hidrolítico. Rev Bras Tecnol Agroindustrial. 2011;5:427-41.

30. Raupp DS, Moreira SS, Banzatto DA, V.C.Sgarbieri. Composição e propriedades fisiológico - nutritivas de uma farinha rica em fibra insolúvel obtida do resíduo fibroso de fecularia de mandioca. Ciência e Tecnol Aliment. 1999;19(2):205-10.

31. Fässler C, Arrigoni E, Venema K, Brouns F, Amadò R. In vitro fermentability of differently digested resistant starch preparations. Mol Nutr Food Res. 2006;50(12):1220-8.

32. Cherbut C, Salvador V, Barry J-L, Doulay F, Delort-Laval J. Dietary fibre effects on intestinal transit in man: involvement of their physicochemical and fermentative properties. Food Hydrocoll. 1991;5(1):15-22.

33. Gray J. Dietary fiber: Definition, analysis, physiology \& health. ILSI Europe Concise Monograph Series; 2006. 1-44 p.

34. Dias LT, Leonel M. Caracterização físico-química de farinhas de mandioca de diferentes localidades do Brasil. Ciência e Agrotecnologia. 2006;30(4):692-700.

35. Nguyen SK, Sophonputtanaphoca S, Kim E, Penner MH. Hydrolytic methods for the quantification of fructose equivalents in herbaceous biomass. Appl Biochem Biotechnol. 2009 Aug;158(2):352-61.

36. Lopes JAS, Li Q, Thompson IP. Biorefinery of waste orange peel. Crit Rev Biotechnol. 2010 Mar;30(1):63-9.

37. Macagnan FT, dos Santos LR, Roberto BS, de Moura FA, Bizzani M, da Silva LP. Biological properties of apple pomace, orange bagasse and passion fruit peel as alternative sources of dietary fibre. Bioact Carbohydrates Diet Fibre. 2015;6(1):1-6.

38. Coelho EM, de Azevedo LC, Viana AC, Ramos IG, Gomes RG, Lima MDS, et al. Physico-chemical properties, rheology and degree of esterification of passion fruit (Passiflora edulis f. flavicarpa) peel flour. J Sci Food Agric. 2018 Jan;98(1):166-73.

39. Cazarin CBB, Silva JK da, Colomeu TC, Zollner R de L, Maróstica Junior MR. Capacidade antioxidante e composição química da casca de maracujá (Passiflora edulis). Ciência Rural. 2014;44:1699-704.

40. Skrovankova S, Sumczynski D, Mlcek J, Jurikova T, Sochor J. Bioactive compounds and antioxidant activity in different types of berries. Int J Mol Sci. 2015 Oct;16(10):24673-706. 


\section{Supplemental material}

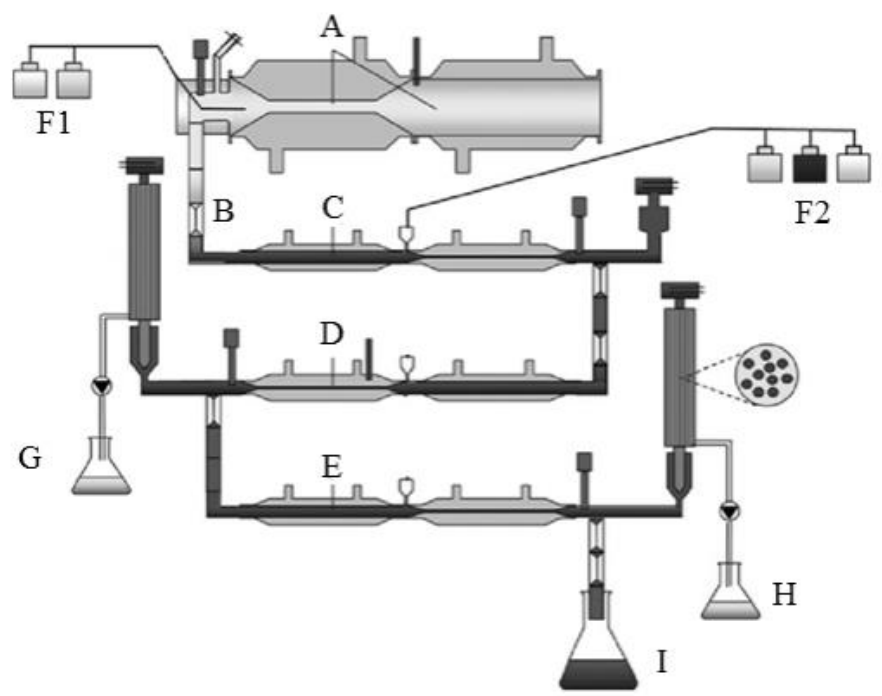

Supplemental Figure 1: TNO intestinal model (TIM-1). Schematic figure of the stomach and small intestine model (TIM-1). $\mathrm{A}=$ stomach compartment; $\mathrm{B}=$ pyloric sphincter; $\mathrm{C}=$ duodenum compartment; $\mathrm{D}=$ jejunum compartment; $\mathrm{E}$ = ileum compartment; $\mathrm{F} 1=$ digestive fluids (saliva with electrolytes and amylase, gastric juice with pepsin lipase and gastric acid); F2 = digestive fluids (pancreatic juice with bicarbonate and digestive enzymes (proteases, lipase, amylase), and bile; $\mathrm{G}=$ jejunum dialysate; $\mathrm{H}=$ ileum dialysate I = ileal efflux. Adapted from Schnorr et al. (28). 


\section{Chapter 3 \\ Prebiotic effects of cassava bagasse in TNO's in vitro model of the colon in lean versus obese microbiota}

Published as:

Bussolo de Souza C, Roeselers G, Troost F, Jonkers D, Koenen ME, Venema K. Prebiotic effects of cassava bagasse in TNO's in vitro model of the colon in lean versus obese microbiota. Journal of Functional Foods. 2014;11(C):210-20. 


\begin{abstract}
Obesity is currently a worldwide epidemic that has serious consequences for human health. It has been suggested that gut microbiota influences body weight, e.g., by producing short-chain fatty acids (SCFA) which are substrates for the host and induce the release of satiety hormones (e.g., PYY). To test the potential prebiotic effect of cassava bagasse (a by-product from cassava flour and starch production) in vitro fermentation experiments were performed using faecal microbiota originating from lean and obese subjects in a validated model of the colon (TIM-2). The microbiota composition and production of microbial metabolites were analysed. Fermentation of cassava bagasse induced different effects in microbiotas originating from lean and obese individuals. It was found to modulate the microbiota composition, since it stimulated the growth of the genera Bifidobacterium and Roseburia in the microbiota originating from lean individuals, and Bifidobacterium, Dorea, Blautia and Coprococcus in the microbiota from obese individuals. Additionally, in the microbiota from lean individuals, fermentation of cassava bagasse generated $57 \%$ of acetate, whilst in the microbiota from obese individuals the production of acetate was $73 \%$. Production of the branched-chain fatty acids iso-butyrate and iso-valerate was higher by the microbiota from lean people $(1.5 \mathrm{mmol}$ and $2.9 \mathrm{mmol}$, respectively) than by the microbiota from obese subjects $(0.52 \mathrm{mmol}$ and $1.3 \mathrm{mmol}$, respectively) upon cassava bagasse fermentation. Overall, the production of SCFA from cassava was similar to that for inulin (positive control), and seemed to drive the obese microbiota composition closer to that of lean individuals. Although supplementary in vivo studies with cassava bagasse are needed, this study shows for the first time the prebiotic potential of cassava bagasse.
\end{abstract}




\section{Introduction}

"Globesity" is the term used to describe the global epidemic of overweight and obesity (1), including occurrence in inhabitants from developing nations like Brazil. Currently, more than 1 billion adults are overweight, of which one-third is clinically obese (2).

In addition to the already known factors that can contribute to obesity, such as genetics and unsuitable dietary habits, new evidence is showing that the gut microbiota can also affect the nutritional metabolism of the host (3). Several studies indicate the role of the gut microbiota in obesity-linked diseases $(4,5)$, although the mechanisms involved are not yet entirely clear.

Short-chain fatty acids (SCFA), mainly acetate, propionate and butyrate, are the major metabolites produced by the microbiota in the large intestine and these deliver additional energy to the host, contributing to about $5-15 \%$ of the total caloric requirements of humans (6). Since even a small daily excess of energy ingestion - as low as $1 \%$ of the recommended daily intake can contribute to increased corporal weight in the long term, all mechanisms modifying foodderived energy availability are important for the balance of body weight (7). This means that the colonic microbiota has a very important role in energy supply.

Besides the extra energy provided by SCFA, it has been hypothesized that these can also influence upper gut motility, satiety and, through this, obesity. Endocrine L-cells present in the colonic mucosa secrete peptides such as peptide YY (PYY), which is involved in appetite regulation (8). PYY release is stimulated directly by nutrients and in response to lipids in distal ileum and colon (9). An in vitro study showed that butyrate increased the expression and release of PYY in epithelial cells (8). Hence, there is some evidence that the effect of fermentable dietary fibre on satiety and body weight could be mediated through the increased colonic production of SCFA (10).

Another mechanism that links obesity and gut microbiota involves the higher amounts of plasma lipopolysaccharide (LPS), called metabolic endotoxemia (11), common in obese and type II diabetic people. LPS is involved in the release of several cytokines that are key factors in activating insulin resistance, and is also considered a factor for the triggering of obesity (11).

Current recommendations for the management of obesity and diabetes propose an increase in consumption of dietary fibre, which may contribute to the control of several metabolic disorders. Dietary fibre is defined as carbohydrate polymers with a degree of polymerization (DP) of three or more, which are neither digested nor absorbed in the human intestine (12). Among the dietary fibres, prebiotics can be used as a tool to modulate the gut microbiota. A 
prebiotic is defined as "a selectively fermented ingredient that allows specific changes, both in the composition and/or activity in the gastrointestinal microbiota that confers benefits upon host well-being and health" (13).

Cassava (Manihot esculenta Crantz) is a tuber, and an original species from Brazil, where the consumption per capita is around $50 \mathrm{~kg} /$ year (14). It can be consumed as natural tuber, nevertheless, as it spoils easily, the production of flour or starch is a manner to conserve it. During the flour or starch processing, a solid residue - called bagasse - is produced, which contains the fibre material from the root and the remaining starch that was not extracted during the manufacturing (15).

To make one ton of starch, around $930 \mathrm{~kg}$ of cassava bagasse is generated and in Brazil, per year, around 97.000 tons of bagasse are produced (16). This residue is considered an industrial waste and it is generally used in animal feed or even disposed of in the environment and thus considered an ecological hazard (17). Since currently it does not have a great value in the market, the main limitation of the commercialization and use of this residue is the drying process, which is considered much too expensive (15).

Cassava bagasse is a good source of fibre and some investigations have demonstrated the beneficial aspects of cassava bagasse intake both in rat and human studies $(18,19)$. These benefits are due to the high insoluble fibre content $(\sim 86 \%$ of the total fibre content) and its associated physiological effects, such as the increase in volume and frequency of defecations - and therefore relief of constipation - and the possibility to be incorporated into food products specially designed for celiac disease patients. However, little is known about the possible prebiotic property of this by-product. A study comparing the fermentability of diverse root crops and legumes using human faecal inoculum revealed that in vitro batch fermentation of the isolated fibre of cassava showed an increased amount of SCFA (20), placing cassava bagasse in a promising position that deserves to be further investigated.

The aim of the present study was to investigate the potential prebiotic effect of cassava bagasse. This was performed assessing the stimulation of growth and/or activity of beneficial colonic bacteria using TNO's dynamic in vitro model of the large intestine (TIM-2) in response to the administration of $7.5 \mathrm{~g} /$ day cassava bagasse over a period of 3 days. The microbiota composition, levels and type of microbial metabolites as well as the levels of LPS were analysed before and after the fermentation experiments. All analyses compared faecal homogenates from lean with those from obese subjects. As a preliminary study, the influence of cassava bagasse on the release of the satiety hormone 
PYY was determined after incubating TIM- 2 samples with porcine intestinal tissue disks.

\section{Materials and methods}

\section{Study design}

Faecal material was collected from lean (body mass index - BMI - 18.5-25 $\mathrm{kg} / \mathrm{m}^{2}$, mean $23.6 \pm 1.4 \mathrm{~kg} / \mathrm{m}^{2}$, age range $23-31$ years, two females $(\mathrm{F})$ and two male $(M)$ ), and obese (BMI $>30 \mathrm{~kg} / \mathrm{m}^{2}$, mean $33.5 \pm 2.6 \mathrm{~kg} / \mathrm{m}^{2}$, age range 23-61 years, $3 \mathrm{~F}$ and $1 \mathrm{M}$ ) volunteers recruited from TNO and Maastricht University Medical Centre, respectively.

The exclusion criteria for both groups included the use of antibiotics or any other medical treatment influencing gut transit or intestinal microbiota during the preceding three months, severe chronic disease, gastrointestinal disease, severe food allergy and intake of probiotics and prebiotics.

In vitro experiments were performed in duplicate with mixed faecal material from either obese or lean individuals in the presence of a) dried and milled cassava bagasse (Grazimara, Biguaçu, Brazil), b) chicory inulin as the "gold standard prebiotic" with purity $\geq 90 \%$ and degree of polymerization $>10$ (Orafti, Amsterdam, the Netherlands), and c) the Simulated Ileum Effluent Medium (SIEM) simulating the material reaching the colon (further on referred to as "standard") - described in detail below.

\section{Faeces collection and standardization}

Faecal samples were collected from each subject. Volunteers were instructed to put the samples in a gastight bag and to place it immediately into a plastic jar containing an anaerocult strip (AnaeroGen ${ }^{\mathrm{TM}}$, Cambridge, UK) to create anaerobic conditions during transport to the anaerobic cabinet and/or freezer $\left(-80^{\circ} \mathrm{C}\right)$. This transport never lasted longer than 3 hours.

The standardized lean or obese microbiota used to inoculate the TIM-2 system was prepared in an anaerobic cabinet (Sheldon Lab - Bactron IV, Cornelius, USA) where the faecal samples (separate for lean and obese individuals) were mixed for standardization (21) and yielded a total of 500 grams of faecal material. Then, $450 \mathrm{~mL}$ of $10 \times$ concentrated dialysis liquid, $2490 \mathrm{~mL}$ of demiwater and $560 \mathrm{~g}$ of glycerol was added.

The faecal material was aliquoted, snap-frozen in liquid nitrogen and stored at $80^{\circ} \mathrm{C}$.

This method was validated previously and showed that standardized stool samples are similar in composition and activity to fresh faecal samples (21). 
Besides that, since every person has a different microbiota composition, by pooling faeces from different people it was possible to: $i$ ) have sufficient amounts of faecal slurry for all experiments and therefore be able to compare the results from the different runs without being cautious about the use of different microbiota (21), and ii) to create a standardized microbiota that encompasses species from different individuals and therefore can be considered to represent a larger population.

\section{TIM-2}

TIM-2 (Supplemental Figure 1) simulates the conditions in the lumen of the human proximal colon and all parameters are computer-controlled (22). The model has been described extensively in recent publications $(23,24)$. In short, it simulates body temperature, $\mathrm{pH}$ in the lumen, composition and rate of secretion, absorption of water and microbial metabolites through a semi permeable membrane inside the model, mixing and transport of the intestinal contents through the simulation of peristaltic movements, and the presence of a complex, high density, metabolically active, anaerobic microbiota of human origin (25).

\section{Experimental setup}

To start the experiment, TIM-2 was inoculated with $70 \mathrm{~mL}$ of the standardized microbiota (described above) plus $50 \mathrm{~mL}$ of dialysis liquid (described below) yielding a total of $120 \mathrm{~mL}$ (total volume of the system). The microbiota adapted to the model conditions with the standard medium (SIEM) for 16 hours (Figure 1) and after that, a period of 4 hours of starvation allowed the bacteria to ferment all available carbohydrates in the system.

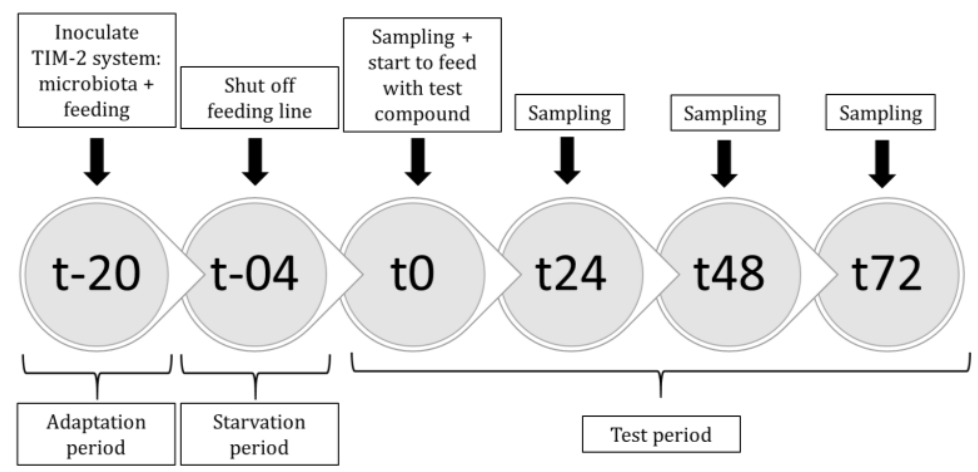

Figure 1: Experimental set-up with timeline for TIM-2 experiments 
Next, samples were collected at time zero ( $\mathrm{t} 0$ ) after which the test compound was added. From this point onwards, a 3-day experimental period started, where the microbiota received the standard feeding (that yields $7.5 \mathrm{~g} /$ day of carbohydrates) or standard feeding without carbohydrate, in which the test compounds were mixed in $(7.5 \mathrm{~g} /$ day of cassava bagasse or inulin), added through the feeding syringe (Supplemental Figure 1g; $2.5 \mathrm{~mL} /$ hour). Samples of the lumen and dialysate were collected at time $0 \mathrm{~h}, 24 \mathrm{~h}, 48 \mathrm{~h}$ and $72 \mathrm{~h}$ (Figure 1).

\section{SIEM and dialysate}

The standard feeding, Simulated Ileal Effluent Medium (SIEM), simulates the material reaching the colon and was slightly modified for experiments in TIM-2 compared to the medium which was described by Gibson et al. (26), mainly concerning a lower amount of water. SIEM contained the following components (g/L): 9.0 pectin, 9.0 xylan, 9.0 arabinogalactan, 9.0 amylopectin, 43.7 casein, 74.6 starch, 31.5 Tween $80,43.7$ bactopepton, 0.7 ox-bile, $4.7 \mathrm{~K}_{2} \mathrm{HPO}_{4} .3 \mathrm{H}_{2} \mathrm{O}, 8.4$ $\mathrm{NaCl}, 0.009 \mathrm{FeSO}_{4 .} .7 \mathrm{H}_{2} \mathrm{O}, 0.7 \mathrm{MgSO}_{4} .7 \mathrm{H}_{2} \mathrm{O}, 0.8 \mathrm{CaCl}_{2} .2 \mathrm{H}_{2} \mathrm{O}, 0.05$ bile, 0.02 haemin and 0.3 cysteine $\cdot \mathrm{HCl}$, plus $1.5 \mathrm{~mL}$ of a vitamin mixture containing (per liter): 1 mg menadione, $2 \mathrm{mg}$ D-biotin, $0.5 \mathrm{mg}$ vitamin B12, $10 \mathrm{mg}$ pantothenate, $5 \mathrm{mg}$ nicotinamide, $5 \mathrm{mg}$ p-aminobenzoic acid and $4 \mathrm{mg}$ thiamine. The $\mathrm{pH}$ was adjusted to 5.8. Dialysis liquid contained (per liter): $2.5 \mathrm{~g} \mathrm{~K}_{2} \mathrm{HPO}_{4} .3 \mathrm{H}_{2} \mathrm{O}, 4.5 \mathrm{~g}$ $\mathrm{NaCl}, 0.005 \mathrm{~g} \mathrm{FeSO}_{4} .7 \mathrm{H}_{2} \mathrm{O}, 0.5 \mathrm{~g} \mathrm{MgSO}_{4} .7 \mathrm{H}_{2} \mathrm{O}, 0.45 \mathrm{~g} \mathrm{CaCl}_{2} .2 \mathrm{H}_{2} \mathrm{O}, 0.05 \mathrm{~g}$ bile and $0.4 \mathrm{~g}$ cysteine $\cdot \mathrm{HCl}$, plus $1 \mathrm{~mL}$ of the vitamin mixture. All medium components were purchased at Tritium Microbiology (Eindhoven, the Netherlands). SIEM only contains indigestible carbohydrates and hence did not require predigestion. SIEM was administered at $60 \mathrm{~mL} /$ day (corresponding to $\sim 7.5 \mathrm{~g}$ carbohydrate/day).

\section{Cassava bagasse}

Cassava bagasse was generated during flour production as described earlier (27). The bagasse was dried by the supplier (Grazimara, Biguaçu, Brazil) in an industrial pan at $250-300{ }^{\circ} \mathrm{C}$ for 20 minutes.

Carbohydrate and protein composition were analyzed at the Food Chemistry Department of Wageningen University. Samples were analysed in duplicate and data are represented as average ( \pm range). Samples contained $0.25 \%$ of nitrogen $(\mathrm{N})$ and $1.7 \%$ of protein. The assay used for protein and nitrogen analyses was the "Dumas" method (28).

Sugar composition was measured by derivatizing the sugars into alditol acetates (28). Briefly, after a pre-hydrolysis step using $72 \% \mathrm{w} / \mathrm{w}$ sulphuric acid at $30{ }^{\circ} \mathrm{C}$ for 1 hour, samples were hydrolyzed with $1 \mathrm{M}$ sulphuric acid at $100{ }^{\circ} \mathrm{C}$ 
for 3 hours in a heating block. Afterwards the sugars were reduced with $\mathrm{NaBH}_{4}$. Acetylation was performed and samples were analysed with gas chromatography (GC) and a flame ionization detector (FID) using inositol as internal standard.

The total uronic acid content was determined with the automated metahydroxydiphenyl assay. Galacturonic acid was used for calibration.

Results are given in $\mathrm{w} / \mathrm{w} \%$ (percentage by weight) and mol \% (molar percentage).

The nature of the fibre (soluble and insoluble portions) as prepared for the fermentation experiments was analysed $(n=4)$ and on average cassava bagasse was composed for $86 \%$ of insoluble fibre.

\section{Microbiota composition}

To determine, among others, whether cassava bagasse would have a prebiotic effect, the composition of the microbiota was evaluated using pyrosequencing of the small subunit ribosomal RNA (16S rRNA) genes present.

DNA was isolated from $200 \mathrm{mg}$ of "faecal" material at the start and at the end of the TIM-2 experiments (t0h and $\mathrm{t} 72 \mathrm{~h}$ ), using a commercial DNA isolation kit (Agowa, Berlin, Germany) following the instructions of the manufacturer.

Generation of PCR amplicon library was performed by amplification of the $16 \mathrm{~S}$ rRNA gene V5-V7 hypervariable region. Amplification was performed using the forward primer 785F (5'GGATTAGATACCCBRGTAGTC-3') and reverse primer 1175R ('5- ACGTCRTCCCCDCCTTCCTC-3). The primers were fitted with the 454 Life Sciences Adapter A (forward primer) and B (reverse primer). The forward primer also included a unique titanium decemnucleotide sample identification key (barcode). The amplification mix contained 2 units of Pfu Ultra II Fusion HS DNA polymerase (Stratagene, La Jolla, CA, USA) and 1x Pfu Ultra II reaction buffer (Stratagene), $200 \mu \mathrm{M}$ dNTP PurePeak DNA polymerase Mix (Pierce Nucleic Acid Technologies, Milwaukee, WI, USA), and $0.2 \mu \mathrm{M}$ of each primer. After an initial denaturation $\left(94^{\circ} \mathrm{C} ; 2 \mathrm{~min}\right), 30 \mathrm{PCR}$ cycles were performed (denaturation $\left(94^{\circ} \mathrm{C} ; 30 \mathrm{sec}\right)$, annealing $\left(50^{\circ} \mathrm{C} ; 40 \mathrm{sec}\right)$, extension $\left(72{ }^{\circ} \mathrm{C} ; 80\right.$ $\mathrm{sec})$ ). Samples with DNA recovery of equal or less than $10 \mathrm{pg} / \mu \mathrm{L}$ of DNA were cycled 35 times using the same protocol. Amplicons were size checked and quantified by gel electrophoresis and Quant-iT Picogreen dsDNA Assay (Invitrogen, Carlsbad, CA - USA) on the Tecan Infinite M200 (Tecan Group Ltd, Männedorf, Switzerland). Amplicons of the individual samples were equimolar pooled and purified from agarose gel by means of QIAquick Gel Extraction Kit Protocol (Qiagen, Hilden, Germany). 
The library was sequenced unidirectionally in the reverse direction (B-adaptor) in one run in the 454 GS-FLX-Titanium Sequencer (Life Sciences [Roche], Branford, CT 06405 USA) by Keygene (Wageningen, the Netherlands).

\section{Sequence processing}

FASTA-formatted sequences and corresponding quality scores were extracted from the .sff data file generated by the GS-FLX-Titatium sequencer using the GS Amplicon software package (Roche, Branford, CT). Sequencing data were processed using modules implemented in the Mothur v. 1.20.0 software platform module (29). Due to the unique barcodes, sequences were binned by sample of origin. For further downstream analyses, barcodes and primer sequences were trimmed, and low quality reads (containing ambiguous base calls $(\mathrm{N})$ in the sequence, $>1$ error in the primer, and $\geq 1$ error in the barcode, more than 8 homopolymers anywhere in the sequence, and the occurrence of a 50 nucleotide window with a window average below 35 , or a length $>500$ bp or $<200 \mathrm{bp}$ and sequences that were too short or too long (automated function)) were removed from the analyses. The data set was simplified by using the "unique.seqs" command to generate a non-redundant (unique) set of sequences. Unique sequences were aligned using the "align.seqs" command and an adaptation of the Bacterial SILVA SEED database as a template (available at: http://www.mothur.org/wiki/Alignment_database). Alignment was performed using the RDP template in order to ensure that comparable regions of the $16 \mathrm{~S}$ rRNA gene across all reads were analysed. Sequences that started before the 2.5-percentile or ended after the 97.5-percentile in the alignment were filtered. Sequences were denoised using the "pre.cluster" command. This command applies a pseudo-single linkage algorithm with the goal of removing sequences that are likely due to pyrosequencing errors (30).

A total of 149.220 potentially chimeric sequences were detected and removed using the "chimera.uchime" command (31). High quality aligned sequences were classified by using the RDP-II naïve Bayesian Classifier database. Aligned sequences clustered into Operational Taxonomic Units (OTUs) using OTU's defined by $97 \%$ similarity, were calculated by the average linkage clustering command method. For each sample rarefaction curves were plotted and community diversity parameters (Shannon diversity index, Chao1 and Simpson's) calculated. Unweighted and weighted Unifrac dendrograms/trees were generated using the Unifrac module implemented in the Mothur software manual and FastTree2 (29) to plot the distance matrices in trees.

Identification at the species level was performed essentially as described before (32). 


\section{SCFA, branched-chain fatty acids (BCFA), lactate and ammonia}

Lumen and spent dialysis liquid from TIM-2 were analysed for microbial metabolites essentially as described before (24). Concentrations were calculated based on a calibration curve that was measured together with each series of samples.

\section{Calculations}

Cumulative amounts of SCFA, BCFA, lactate and ammonia were calculated considering the measurements in spent dialysis liquid and luminal content and the volume of each sample.

\section{Energy production}

Energy production in the form of SCFA was calculated using the following values for acetate, propionate, butyrate and lactate respectively: 874, 1536, 2192 , and $1364 \mathrm{~kJ} \mathrm{~mol}^{-1}$ (33).

\section{LPS}

Lumen samples from TIM-2 (0h and 72h) were analysed for LPS as follows. Samples were centrifuged for 10 minutes at $10.000 \mathrm{rpm}$ at room temperature. The supernatant - $100 \mu \mathrm{L}$ - was diluted eight-fold with endotoxin-free phosphate buffered saline (PBS - Gibco by Life Technologies, UK) and passed through a disposable $0.20 \mu \mathrm{m}$ sterile, pyrogen-free filter (Minisart, Sartorius Stedim Biotech, Germany). Samples were heated at $100{ }^{\circ} \mathrm{C}$ for 30 minutes and centrifuged for 2 minutes at $10.000 \mathrm{rpm}$ at room temperature (34). Afterwards they were further diluted 1000 -fold using PBS.

The assay was performed using a Limulus Amebocyte Lysate (LAL) kit (Thermo Scientific, Rockfort, IL, USA) in a 96-well microplate (Sarstedt Inc., Newton, USA) according to the instruction of the manufacturer. The absorbance was read at $405 \mathrm{~nm}$ using a microplate reader (Biotek Synergy HT, Vermont, USA)(34).

\section{Statistical analysis}

Data are represented as average ( \pm range). Since the system yields highly reproducible results, the experiments were performed in duplicate. Due to this it was not possible to perform statistical analyses. 


\section{Results}

\section{Microbiota composition}

All the baseline (time zero) samples clustered together (Figure 2), showing that all fermentations started with the same microbiota composition, although those of the lean microbiota clustered separately from those of the obese microbiota (Supplemental Figure 2).

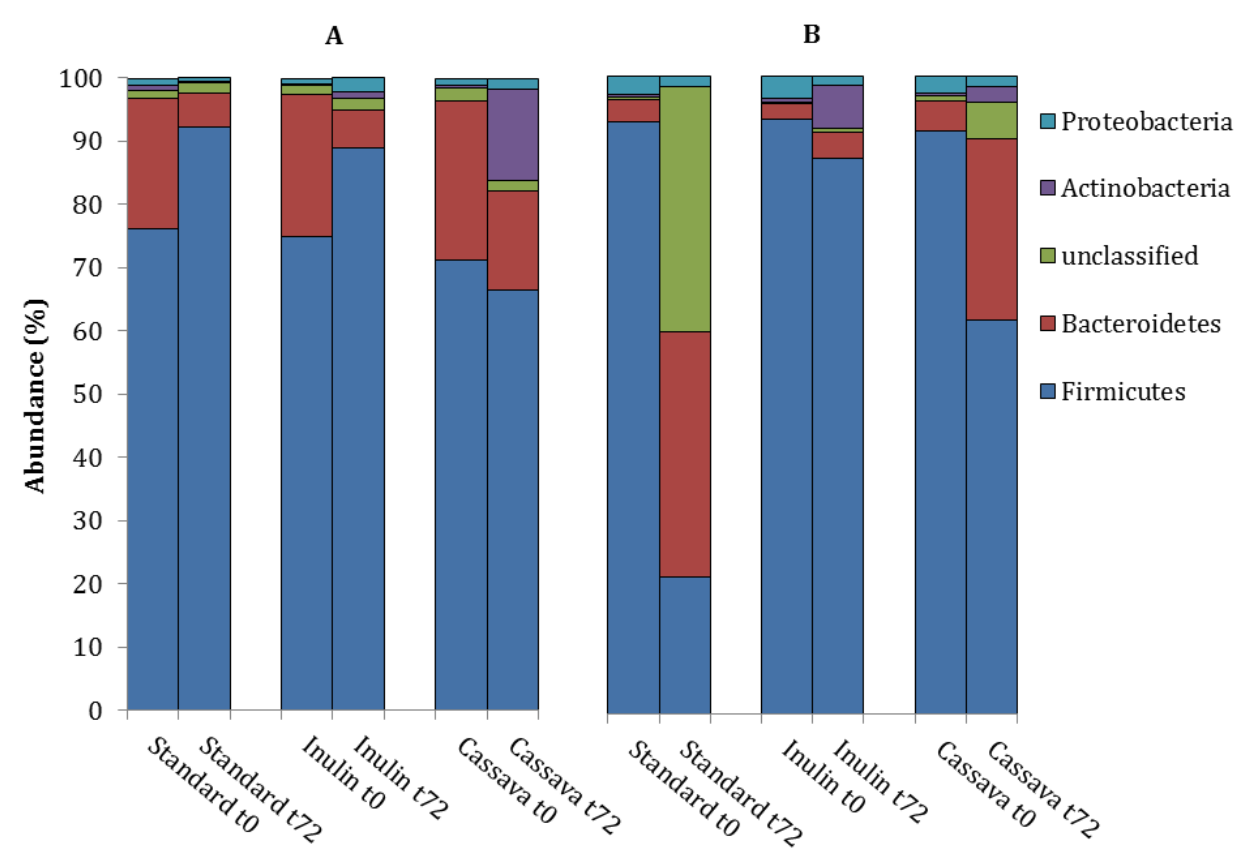

Figure 2: Percentage of each phylum at time $0 \mathrm{~h}$ and $72 \mathrm{~h}$ with standard, inulin and cassava in lean (A) and obese (B) microbiotas.

\section{Lean microbiota}

Firmicutes increased $16 \%$ and $13 \%$ for standard and inulin, respectively, and decreased $4.6 \%$ for cassava (Figure 2A). The final abundance was $92 \%$, 89\% and $67 \%$ for standard, inulin and cassava, respectively.

After 72 hours the phylum Bacteroidetes had decreased in all groups. It decreased by $15 \%, 16 \%$ and $9.5 \%$ in standard, inulin and cassava, respectively. The final abundance was $5.3 \%$ for standard, $6.1 \%$ for inulin and $16 \%$ in cassava.

Actinobacteria (including the genus Bifidobacterium) decreased $0.70 \%$ in standard, resulting in a final abundance of $0.04 \%$. Inulin caused a subtle increase $(0.74 \%)$, while cassava showed the highest increase of Actinobacteria (14\%), with a final abundance of $15 \%$. 


\section{Obese microbiota}

The obese microbiota (Figure 2B) showed a higher percentage of Firmicutes at time zero when compared with the lean microbiota. However, it decreased in all groups - by $71 \%, 6.1 \%$ and $29 \%$ to a final abundance of $22 \%, 87 \%$ and $62 \%$ for standard, inulin and cassava.

Bacteroidetes showed a major increase in abundance under standard condition. The initial percentage was $3.6 \%$ while at the end of the fermentation period this increased to $34 \%$. A similar tendency was seen for cassava, where the initial amount of Bacteroidetes was $4.8 \%$ before and $28 \%$ after the fermentation period. The abundance of this phylum on inulin did not change much over the fermentation period.

Actinobacteria showed a higher increase in the obese microbiota when fed with inulin compared with standard and cassava. The initial abundance of this phylum was $0.74 \%$ and it increased with $6.2 \%$. Cassava also showed a slight increase in this phylum, where the initial proportion was $0.43 \%$ and the final was $2.6 \%$. In the standard, Actinobacteria did not differ after 72 hours of experiment. There were very few changes in the other phyla.

\section{SCFA and lactate}

Figure 3 shows the average of total cumulative production of the beneficial microbial metabolites acetate, propionate, butyrate and lactate during the TIM2 experiments by the lean microbiota (A) and obese microbiota (B).

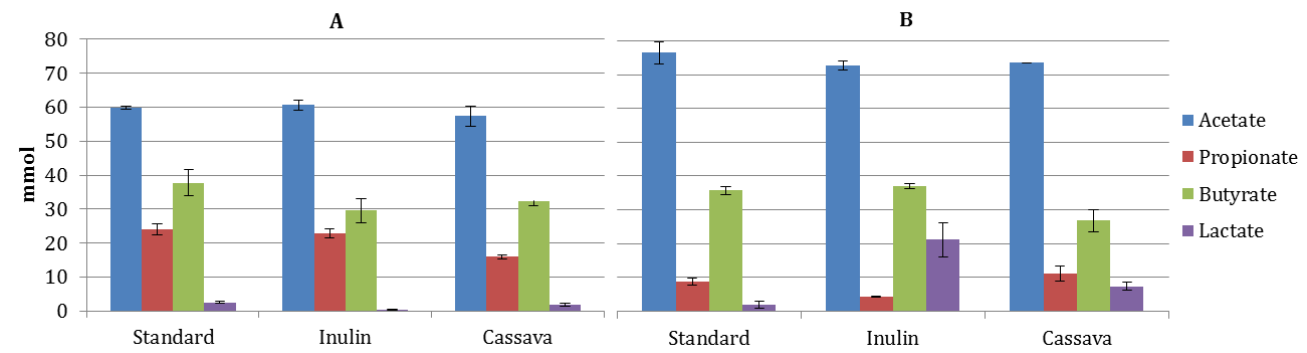

Figure 3: Average cumulative SCFA and lactate production (mmol) by the lean (A) and obese (B) microbiotas after 72 hours $(\mathrm{n}=2 \pm$ range).

In lean microbiota, acetate production was comparable for the three substrates. Standard showed higher amounts of butyrate, and propionate production was lower than that of other SCFA. Lactate production was higher in the standard, followed by cassava and inulin.

In obese microbiota, acetate production was also comparable for the three substrates, but with higher values than observed for the lean microbiota. Fermentation of cassava showed higher production of propionate and the 
lowest production of butyrate. Lactate production in obese microbiota was higher for inulin and cassava when compared with lean microbiota.

Supplemental Figure 3 shows the kinetics of the cumulative average production of SCFA on the cassava substrate during the entire test period for both microbiotas.

\section{Production of energy}

In lean microbiota, standard showed the highest production of energy in $\mathrm{kJ}$ in the form of SCFA and lactate - $176 \mathrm{~kJ}$. Fermentation of inulin by the lean microbiota produced less energy compared to the obese microbiota $(153 \mathrm{~kJ}$ versus $180 \mathrm{~kJ}$ ). Lean and obese microbiota extracted similar amounts of energy from cassava (148 kJ versus $151 \mathrm{~kJ}$ ), which was overall lower than for the other substrates.

\section{BCFA and ammonia}

Table 1 shows the cumulative production (mmol) of BCFA - iso-butyrate and iso-valerate - and ammonia at $\mathrm{t} 72$ by lean and obese microbiota.

Table 1: Cumulative production of BCFA (iso-butyrate and iso-valerate - mmol) and ammonia (mmol) by lean and obese microbiotas after 3 days of addition of the test compounds at $7.5 \mathrm{~g} /$ day $(\mathrm{n}=2)$.

\begin{tabular}{l|lll|lll}
\hline & \multicolumn{3}{|c|}{ Lean } & \multicolumn{3}{c}{ Obese } \\
\hline & $i$-butyrate & $i$-valerate & ammonia & $i$-butyrate & $i$-valerate & ammonia \\
Standard & $1.3 \pm 0.04$ & $2.6 \pm 0.05$ & $65.2 \pm 3.08$ & $0.13 \pm 0.18$ & $0.53 \pm 0.31$ & $41.8 \pm 2.07$ \\
Inulin & $1.0 \pm 0.04$ & $2.1 \pm 0.01$ & $51.2 \pm 1.27$ & $0.13 \pm 0.13$ & $0.42 \pm 0.33$ & $39.1 \pm 0.20$ \\
Cassava & $1.5 \pm 0.09$ & $2.9 \pm 0.22$ & $60.5 \pm 9.23$ & $0.52 \pm 0.33$ & $1.3 \pm 0.49$ & $55.8 \pm 4.9$ \\
\hline
\end{tabular}

In the lean microbiota, the production of BCFA was slightly higher after fermentation with cassava. With regards to ammonia production, microbiota fed with standard produced the highest amount.

In the obese microbiota, fermentation of cassava resulted in the highest production either of $i$-butyrate, $i$-valerate and ammonia when compared with the standard and inulin.

\section{LPS}

Figure 4 shows that in the lean microbiota, after a fermentation period of 72 hours, the luminal LPS concentration on all substrates had increased in similar amounts. 


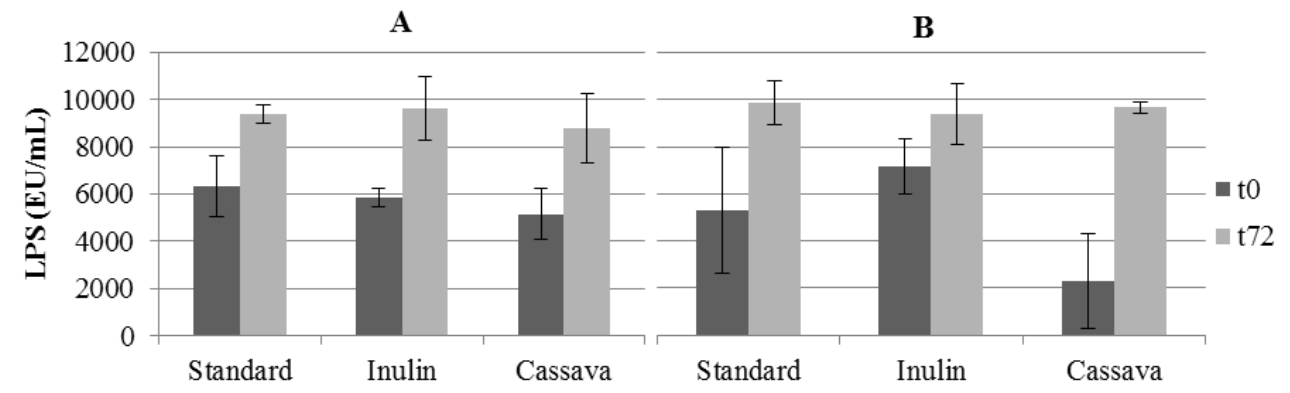

Figure 4: LPS concentration (endotoxin units (EU)) in the TIM-2 lumen samples at time $0 \mathrm{~h}$ and $72 \mathrm{~h}$ of fermentation with different substrates with lean (A) and obese (B) microbiotas.

In obese microbiota the initial amount of LPS on cassava was lower compared with the other substrates, however, it presented the highest increase.

\section{Discussion}

\section{Cassava bagasse composition}

As can be observed in Table 2, cassava bagasse is predominantly composed of glucose, probably due the fact that the manufacturing process is not able to extract all the starch present. Resistant starch is indigestible in the small intestine, and it enters the large intestine. It has been proposed that resistant starch has properties similar to dietary fibre and thus could also affect satiety and have a beneficial role in weight regulation (35). Initial experiments to determine the digestibility of the starch have been carried out and the results show that the starch in cassava bagasse is partly digestible. Therefore, to reach the colon delivery strategies (such as encapsulation, etc.) may have to be devised.

Table 2: Sugar composition of cassava bagasse

\begin{tabular}{lcccccc}
\hline & Arabinose & Xylose & Mannose & Galactose & Glucose & Uronic acid \\
\hline mol \% & 0.5 & 0.5 & 0 & 1 & 96 & 2 \\
w/w \% & 0.4 & 0.4 & 0.2 & 0.7 & 85 & 2 \\
\hline
\end{tabular}

${ }^{*} \mathrm{w} / \mathrm{w}$ does not sum up to $100 \%$ due to the presence of moisture.

\section{Microbiota composition}

The microbiota composition in the beginning of the TIM-2 experiments (Figure 2 ) is in accordance with the finding of Gordon and colleagues (36), with an increased abundance of the phylum Bacteroidetes and decreased abundance of Firmicutes in lean microbiota when compared with obese microbiota. It has been hypothesized that an increased ratio of Firmicutes to Bacteroidetes may 
have a significant contribution to the pathophysiology of obesity through the promotion of adiposity or could even represent a host-mediated adaptive response to limit energy uptake/storage through, for instance, reducing the capacity to ferment polysaccharides (36). On the one hand is not logical to link this ratio with obesity because it is known that Bacteroidetes, especially members of the genus Bacteroides, have a great capacity to metabolize an enormous variety of glycans from plants and animals (37) and therefore could provide more energy in the form of SCFA and also monosaccharides that can be absorbed by the human gut. On the other hand, it has been proposed that SCFA have satiety-enhancing properties (38) and therefore it could be hypothesized that the higher the amounts of SCFA produced, the more satiety effects would occur (and thus the leaner one could become). Additionally, research showed an increase in Bacteroidetes in overweight and obese individuals (39), which is the opposite of the results published by Gordon and colleagues. Moreover, these phyla are composed of several dozens of species, and the role in obesity of the species that represent these phyla in the gut eco-system has not been examined in these early studies.

Cassava had some unique effects regarding the abundance of some species. In lean microbiota abundance of Ruminococcus obeum and Bacteroides uniformis increased, and Eubacterium rectale and Prevotella copri decreased upon cassava bagasse feeding.

Inulin also had some unique effects, which in lean microbiota included the increase of Catenibacterium mitsuokai and decrease of Eubacterium rectale and Ruminococcus bromii. In obese microbiota it increased the abundance of Eubacterium hadrum, Lactococcus garvieae and Eubacterium biforme.

In lean microbiota, both inulin and cassava stimulated the increase in abundance of the genus Bifidobacterium. However, while inulin increased the relative proportion by a factor of 169-fold compared to the standard (Supplemental Figure 4), cassava showed a greater response with an overall 1738-fold increase. Interestingly, different effects were observed in obese microbiota. After 72 hours of fermentation with inulin and cassava, inulin showed a stronger effect on Bifidobacterium than cassava, with an 80-fold increase relative to the standard. Cassava, although also stimulating the increase of this genus by 42 -fold, had a clearly less strong effect when compared with lean microbiota. Obese samples become rather similar to lean samples after 72 hours (Supplemental Figure 2), indicating the microbiota becomes healthier.

The Bifidobacterium and Lactobacillus genera are used to characterize the prebiotic effect of substrates, reflecting their health-promoting properties such 
as inhibition of opportunistic pathogens (40). Cassava showed a greater increase in the genus Bifidobacterium in the microbiota obtained from lean individuals than the well-known bifidogenic prebiotic inulin. Cassava also induced the relative contribution of bifidobacteria in microbiota obtained from obese individuals, but to a lesser extent when compared with lean individuals. Specifically, either in lean or obese microbiota at $\mathrm{t} 72 \mathrm{~h}$, Bifidobacterium dentium was the most dominant Bifidobacterium present. B. adolescentis, B. breve and $B$. pseudocatenulatum increased in lean and obese microbiota. The effects of inulin and cassava on the genus Lactobacillus did not differ from the standard, either in lean or obese microbiota.

The genus Roseburia is related to butyrate production and is thus thought to play a key role in colonic health (5). In comparison with the standard, cassava was able to increase the relative proportion of Roseburia 7-fold after 72 hours of fermentation in lean microbiota (Supplemental Figure 4), especially Roseburia faecis. Interestingly, inulin decreased the amount of Roseburia in lean microbiota 7 -fold. There were no changes in this genus in microbiota obtained from obese individuals after 72 hours of fermentation.

Cassava bagasse had the ability to increase the amount of Bacteroides in lean microbiota compared to the standard, whereas the amount in obese microbiota was not influenced compared to the standard. Known propionate producers belong to the genus Bacteroides (39) and besides that, large polysaccharides, such as resistant starch, are more readily broken down by Bacteroides, while bifidobacteria prefer to ferment shorter-chain oligosaccharides (24). Interestingly, bifidobacteria and Bacteroides were higher in lean microbiota after feeding cassava, indicating that cross-feeding between these two genera may have occurred (24). Bifidobacteria have been shown to be stimulated upon starch feeding before (23).

The genera Dorea and Coprococcus were stimulated 44 and 14-fold, respectively, by cassava (Supplemental Figure 4). Unfortunately, these genera have only recently been described as being part of the microbiota, and their importance to host physiology is not yet known. Dorea has been found to be stimulated by inulin (41). In our study, this is only observed in the faecal material of obese individuals. In the faecal material from lean subjects, Dorea actually decreased upon inulin feeding. There were very few changes in the other phyla. As expected the Verrucomicrobia, containing Akkermansia (Akkermansia muciniphila is inversely correlated with weight gain (10)), decreased, as there was no mucin added to TIM-2, which is the major substrate of the genus. 


\section{SCFA and lactate}

It is clear that acetate production by the obese microbiota, for all substrates, was much higher than production of the other two SCFA (Figure 3 and S4). Acetate stimulates lipid synthesis, but at the same time propionate may counteract de novo lipogenesis from acetate (42). Therefore, it can be speculated that the ratio of SCFA produced by the obese microbiota is in a sense not "protective" against excess lipid production, since acetate concentrations are higher than those of propionate. Indeed SCFA have different metabolic effects in the host and therefore changing the proportional abundance of these metabolites may result in significant changes in responses of host cells (43).

It is noteworthy that lactate production by the obese microbiota fed with inulin was higher than on all the other substrates, as well as compared with lean microbiota. Lactate is an intermediate metabolite in intestinal fermentation and it only accumulates when there is a fast fermentation of the substrate (24). Besides that, it is the major end-product of bifidobacterial metabolism, which can explain the amount of lactate production in obese microbiota fed with inulin since it primarily stimulated the increase of abundance of this genus. However, cassava bagasse in lean microbiota increased the abundance of bifidobacteria even more, but was not followed by an increase in either acetate or lactate. Here, lactate may have been converted into the other SCFA.

\section{BCFA and ammonia}

The amounts of BCFA produced during cassava fermentation by the lean microbiota were higher when comparing with the standard and inulin. These concentrations were higher when compared with the obese microbiota. We hypothesize that the difference in microbiota composition between lean and obese may affect the production of these different metabolites. Further studies are required to test this hypothesis and find out exactly which bacterial species are related with protein fermentation that in turn yields BCFA and ammonia as metabolites.

\section{LPS}

Given that obesity is considered to be a low grade inflammatory disease and that a previous study demonstrated that obese subjects have higher concentrations of plasma LPS (11) than lean subjects, it was hypothesized that the luminal concentration of LPS in TIM-2 could also be higher for the microbiota from obese subjects compared with the microbiota from lean subjects. Our first results shown here are not in agreement with this. Further experiments are required to confirm differences in luminal LPS concentration. 


\section{PYY}

In an ex vivo experiment, porcine intestinal tissue disks were incubated with TIM-2 luminal samples and preliminary results indicated a higher release of PYY induced by the obese microbiota metabolites (not shown). Since PYY induces satiety, this was unexpected. However, it may also point to the beneficial effects of dietary interventions with inulin and/or cassava on PYY expression. It remains to be seen which (combination of) metabolites in the obese samples are responsible for the higher release of PYY from colonic tissue.

\section{Production of energy}

It was expected that the results of this study would show a higher amount of SCFA produced by the obese microbiota when compared with the lean microbiota, since SCFA provide extra energy to the host. However, the present study did not show this. Inulin was the only substrate that after fermentation by the obese microbiota showed a higher amount of energy extraction when compared with the lean microbiota. It should be noted that equal amounts of carbohydrates were provided to these microbiotas, which is likely to be different in vivo, where obese individuals tend to overeat and hence more carbohydrates may reach the colon.

This unexpected result may reflect the satiety-enhancing properties of SCFA, since the expression of proglucagon, the precursor of glucagon-like peptide 1 (GLP-1), can be up-regulated by SCFA (44). Besides this, it has been reported that butyrate can increase energy expenditure and fatty acid oxidation in rats (45). Thus, one can argue that higher amounts of SCFA are responsible to keep the lean individuals lean due to the satiety effects of the gut microbiota metabolites. The results clearly show that SCFA production depends on the substrate provided and on the origin of the microbiota. Accordingly, this study shows that microbiota fermentation of substrates resulting in production of SCFA (and therefore the production of extra energy to the host) is not the only factor that affects body weight. These other factors remain to be investigated. It is definitely not only a question of extra calories yielded, but the intricate role that each SCFA has on host metabolism. 


\section{Conclusions}

Health professionals know the difficulties that people experience in changing eating habits and life style necessary for weight management. Therefore, there is an urgent need for alternative solutions that can assist people in their daily battle with overweight and obesity and by this improve the quality of life of a relatively large portion of the world population.

Here we showed for the first time the potential prebiotic properties of cassava bagasse, an industrial residue that currently does not have commercial value. It induced different effects in microbiotas originating from lean and obese individuals, and modified the obese microbiota composition closer to that of lean individuals. It is thought that particularly the cassava bagasse starch fraction was responsible for the increase in growth of beneficial bacteria, including the bifidogenic effect. It thus shows great promise in becoming a functional food in the future, although further experiments are required to elucidate the mechanisms involved in more detail. According EFSA recommendations it also is necessary to show these prebiotic effects of cassava bagasse in supplementary in vivo studies. The methodology used here shows great promise as a rapid screening tool for evaluation of prebiotic activity of indigestible substrates.

\section{Acknowledgments}

We acknowledge Christiane Rosch and Henk Schols from Wageningen University for the sugar and protein analysis. Wendy Borst from TNO for technical support with TIM-2, Tom Gorissen and Hans Kooijman from TNO for helping with SCFA analysis. Marcus de Goffau from University of Groningen for the bacterial species analysis. The Brazilian company (Alimentos Grazimara Ltda.) for sending samples of cassava bagasse, and volunteers that donated faeces and made this study possible.

\section{Financial support}

Carlota Bussolo de Souza received a scholarship from CNPq (The National Council for Scientific and Technological Development) - Brazil - under the Program "Science without Borders" (246027/2012-6). TNO financed the experiments and analyses.

\section{Conflict of Interest}

The authors declare that there is no conflict of interest.

Part of the results was presented at the European Network for Gastrointestinal Health Research (ENGIRH) 2013, 18 ${ }^{\text {th }}-20^{\text {th }}$ August - Valencia, Spain, at the $15^{\text {th }}$ 
Chapter 3

Gut Day Symposium (2013), $7^{\text {th }}$ November - Groningen, the Netherlands and at the $13^{\text {th }}$ European Training Course on Carbohydrates (2014), $13^{\text {th }}-17^{\text {th }}$ April Wageningen, the Netherlands. 


\section{References}

1. Elli M, Colombo 0, Tagliabue A. A common core microbiota between obese individuals and their lean relatives? Evaluation of the predisposition to obesity on the basis of the fecal microflora profile. Med Hypotheses. 2010;75(4):350-2.

2. WHO. Obesity and Overweight - Fact sheet n. 311. 2017.

3. Fleissner CK, Huebel N, Abd El-Bary MM, Loh G, Klaus S, Blaut M. Absence of intestinal microbiota does not protect mice from dietinduced obesity. Br J Nutr. 2010;104(6):919-29.

4. Backhed F, Ding H, Wang T, Hooper L V, Koh GY, Nagy A, et al. The gut microbiota as an environmental factor that regulates fat storage. Proc Natl Acad Sci U S A. United States; 2004 Nov;101(44):15718-23.

5. Venema K. Role of gut microbiota in the control of energy and carbohydrate metabolism. Curr Opin Clin Nutr Metab Care. 2010;13(4):432-8.

6. Blaut M, Klaus S. Intestinal microbiota and obesity. Handbood Exp Pharmacol. 2012;209:251-73.

7. Cani PD, Delzenne NM, Amar J, Burcelin R. Role of gut microflora in the development of obesity and insulin resistance following high-fat diet feeding. Pathol Biol (Paris). France; 2008 Jul;56(5):305-9.

8. Hamer HM, Jonkers D, Venema K, Vanhoutvin S, Troost FJ, Brummer R-J. Review article: the role of butyrate on colonic function. Aliment Pharmacol Ther. England; 2008 Jan;27(2):104-19.

9. Voortman T, Hendriks HFJ, Witkamp RF, Wortelboer HM. Effects of longand short-chain fatty acids on the release of gastrointestinal hormones using an ex vivo porcine intestinal tissue model. J Agric Food Chem. 2012;60(36):9035-42.

10. Delzenne NM, Neyrinck AM, Cani PD. Gut microbiota and metabolic disorders: How prebiotic can work? Br J Nutr. 2013;109(2).

11. Cani PD, Amar J, Iglesias MA, Poggi M, Knauf C, Bastelica D, et al. Metabolic endotoxemia initiates obesity and insulin resistance. Diabetes. United States; 2007 Jul;56(7):1761-72.

12. Stephen AM, Champ MM-J, Cloran SJ, Fleith M, van Lieshout L, Mejborn H, et al. Dietary fibre in Europe: current state of knowledge on definitions, sources, recommendations, intakes and relationships to health. Nutr Res Rev. Cambridge University Press; 2017 Jul 5;1-42.

13. Gibson GR, Roberfroid MB. Dietary modulation of the human colonic microbiota: introducing the concept of prebiotics. J Nutr. 1995;125(6):1401-12.

14. Cardoso EMR, Hühn S, Junior J de DN. Processo industrial para beneficiamento da macaxeira. Novos Cad NAEA. Pará; 1999;2(2):177-84.

15. Rodrigues JPM, Caliari M, Asquieri ER. Caracterização e análise sensorial de biscoitos de polvilho elaborados com diferentes níveis de farelo de mandioca. Ciência Rural. 2011;41(12):2196-202.

16. Leonel M, Cereda MP, Roau X. Aproveitamento do resíduo da produção 
de etanol a partir de farelo de mandioca, como fonte de fibras dietéticas. Ciência e Tecnol Aliment. 1999;19(2):241-5.

17. Ferreira GDG, Oliveira RL, Cardoso E da C, Magalhães ALR, Brito EL. Valor nutritivo de co-produtos da mandioca. Rev Bras Saúde e Produção Anim. 2007;8(4):364-74.

18. Costa LA da, Bramorski A, Silva MC, Teixeira E, Amboni R. Desenvolvimento de alimento em barra a base de resíduo da fabricação de farinha de mandioca. Aliment e Nutr. 2005 Mar 1;16(4):389-96.

19. Osundahunsi OF, Williams AO, Oluwalana IB. Prebiotic effects of cassava fibre as an ingredient in cracker-like products. Food Funct. 2012;3(2):159-63.

20. Mallillin AC, Trinidad TP, Raterta R, Dagbay K, Loyola AS. Dietary fibre and fermentability characteristics of root crops and legumes. Br J Nutr. 2008;100(3):485-8.

21. Venema K, Nuenen M van, Smeets-Peeters M, Minekus M, Havenaar R. TNO's in vitro large intestinal model: an excellent screening tool for functional food and pharmaceutical research. Ernährung Nutr. 2000;24(12):559-64.

22. Minekus M, Smeets-Peeters M, Bernalier A, Marol-Bonnin S, Havenaar R, Marteau $\mathrm{P}$, et al. A computer-controlled system to simulate conditions of the large intestine with peristaltic mixing, water absorption and absorption of fermentation products. Appl Microbiol Biotechnol. 1999;53(1):108-14.

23. Kovatcheva-Datchary P, Egert $M$, Maathuis A, Rajilic-Stojanovic M, de Graaf AA, Smidt $\mathrm{H}$, et al. Linking phylogenetic identities of bacteria to starch fermentation in an in vitro model of the large intestine by RNAbased stable isotope probing. Environ Microbiol.2009;11(4):914-26.

24. Maathuis A, Hoffman A, Evans A, Sanders L, Venema K. The effect of the undigested fraction of maize products on the activity and composition of the microbiota determined in a dynamic in vitro model of the human proximal large intestine. J Am Coll Nutr. 2009;28(6):657-66.

25. Reimer RA, Maathuis AJ, Venema K, Lyon MR, Gahler RJ, Wood S. Effect of the novel polysaccharide PolyGlycopleX(R) on short-chain fatty acid production in a computer-controlled in vitro model of the human large intestine. Nutrients. 2014;6(3):1115-27.

26. Gibson GR, Cummings JH, Macfarlane GT. Use of a three-stage continuous culture system to study the effect of mucin on dissimilatory sulfate reduction and methanogenesis by mixed populations of human gut bacteria. Appl Environ Microbiol. 1988;54(11):2750-5.

27. Pandey A, Soccol CR, Nigam P, Soccol VT, Vandenberghe LPS, Mohan R. Biotechnological potential of agro-industrial residues. II: cassava bagasse. Bioresour Technol. 2000;74(1):81-7.

28. Pustjens AM, De Vries S, Gerrits WJ, Kabel MA, Schols HA, Gruppen H. Residual carbohydrates from in vitro digested processed rapeseed (Brassica napus) meal. J Agric Food Chem. 2012;60(34):8257-63. 
29. Schloss PD, Westcott SL, Ryabin T, Hall JR, Hartmann M, Hollister EB, et al. Introducing mothur: open-source, platform-independent, communitysupported software for describing and comparing microbial communities. Appl Environ Microbiol. 2009;75(23):7537-41.

30. Huse SM, Welch DM, Morrison HG, Sogin ML. Ironing out the wrinkles in the rare biosphere through improved OTU clustering. Environ Microbiol. 2010;12(7):1889-98.

31. Edgar RC, Haas BJ, Clemente JC, Quince C, Knight R. UCHIME improves sensitivity and speed of chimera detection. Bioinformatics. 2011;27(16):2194-200.

32. de Goffau MC, Luopajarvi K, Knip M, Ilonen J, Ruohtula T, Harkonen T, et al. Fecal microbiota composition differs between children with beta-cell autoimmunity and those without. Diabetes. 2013;62(4):1238-44.

33. van der Kamp JW, Jones J, McCleary B, Topping D. Dietary fibre: New frontiers for food and health. Wageningen Academic Publishers; 2010. $592 \mathrm{p}$.

34. Gozho GN, Krause DO, Plaizier JC. Rumen lipopolysaccharide and inflammation during grain adaptation and subacute ruminal acidosis in steers. J Dairy Sci. 2006;89(11):4404-13.

35. Topping DL, Clifton PM. Short-chain fatty acids and human colonic function: roles of resistant starch and nonstarch polysaccharides. Physiol Rev.2001;81(3):1031-64.

36. Ley RE, Backhed F, Turnbaugh P, Lozupone CA, Knight RD, Gordon JI. Obesity alters gut microbial ecology. Proc Natl Acad Sci U S A. 2005;102(31):11070-5.

37. Martens EC, Lowe EC, Chiang H, Pudlo NA, Wu M, McNulty NP, et al. Recognition and degradation of plant cell wall polysaccharides by two human gut symbionts. PLoS Biol. 2011;9(12):e1001221.

38. Darzi J, Frost GS, Robertson MD. Do SCFA have a role in appetite regulation? Proc Nutr Soc. 2011;70(1):119-28.

39. Schwiertz A, Taras D, Schafer K, Beijer S, Bos NA, Donus C, et al. Microbiota and SCFA in lean and overweight healthy subjects. Obes (Silver Spring). 2010;18(1):190-5.

40. Rycroft CE, Jones MR, Gibson GR, Rastall RA. A comparative in vitro evaluation of the fermentation properties of prebiotic oligosaccharides. J Appl Microbioly. 2001;91(5):878-87.

41. Kovatcheva-Datchary P, Egert M, Maathuis A, Graaf AA de, Smidt H, Vos WM de, et al. Analyzing the functionality of the human intestinal microbiota by stable isotope probing. Vol. PhD thesis, The Netherlands: University of Wageningen. [Wageningen]; 2010.

42. Delzenne NM, Cani PD. Interaction between obesity and the gut microbiota: relevance in nutrition. Annu Rev Nutr. 2011;31:15-31.

43. Yadav H, Lee JH, Lloyd J, Walter P, Rane SG. Beneficial metabolic effects of a probiotic via butyrate-induced GLP-1 hormone secretion. J Biol Chem. 2013;288(35):25088-97. 
Chapter 3

44. Parnell JA, Reimer RA. Prebiotic fibres dose-dependently increase satiety hormones and alter Bacteroidetes and Firmicutes in lean and obese JCR:LA-cp rats. Br J Nutr. 2012;107(4):601-13.

45. Gao Z, Yin J, Zhang J, Ward RE, Martin RJ, Lefevre M, et al. Butyrate improves insulin sensitivity and increases energy expenditure in mice. Diabetes. 2009;58(7):1509-17. 


\section{Supplementary material}

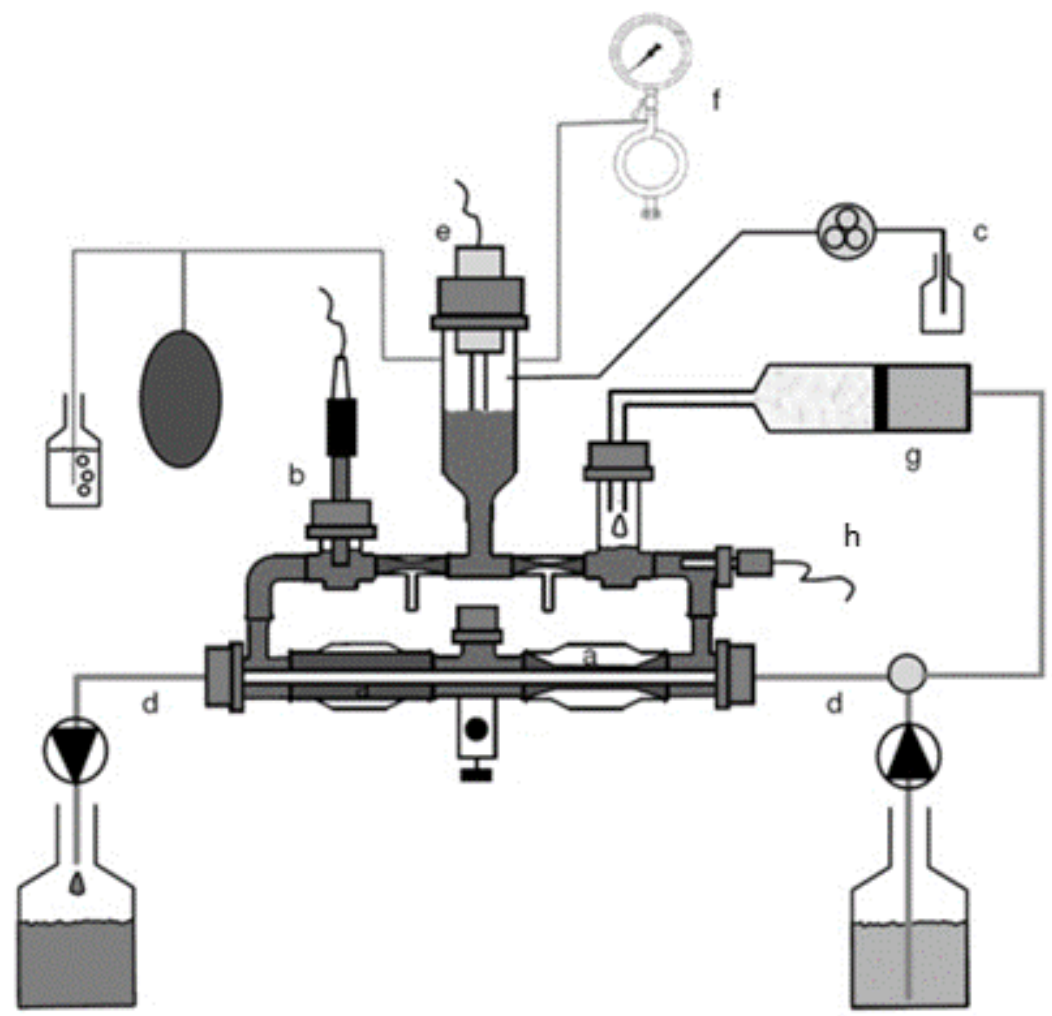

Supplemental Figure 1: TNO's in vitro model of the proximal large intestine (TIM-2). a: mixing units; b: $\mathrm{pH}$ electrode; c: alkali circuit; d: dialysis circuit (d1: dialysis liquid in; $\mathrm{d} 2$ : spent dialysis liquid); e: level sensor; f: nitrogen gas inlet; g: food syringe, h: temperature sensor. The total volume of the system is circa $120 \mathrm{~mL} \mathrm{(22).}$ 
A

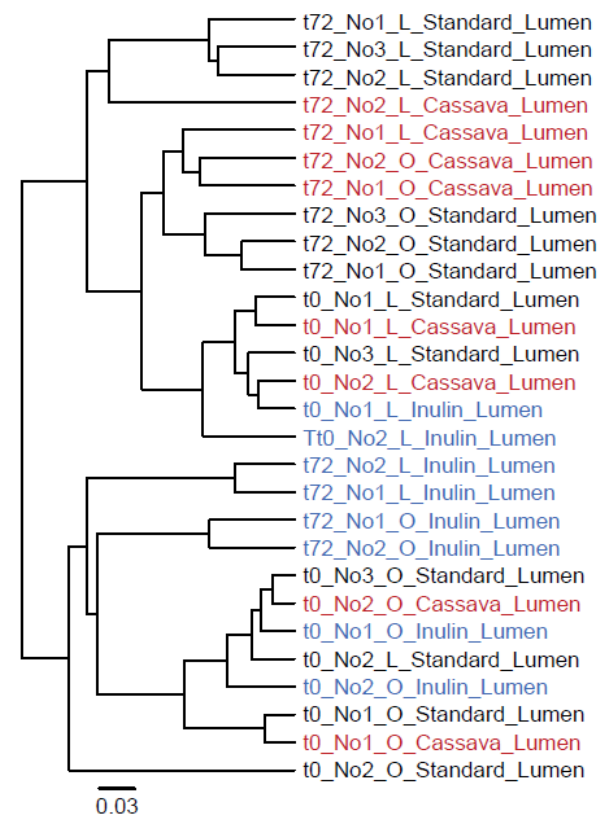

B
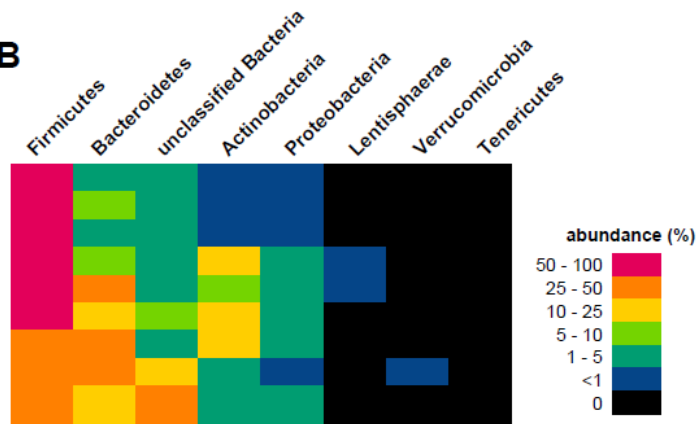

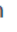

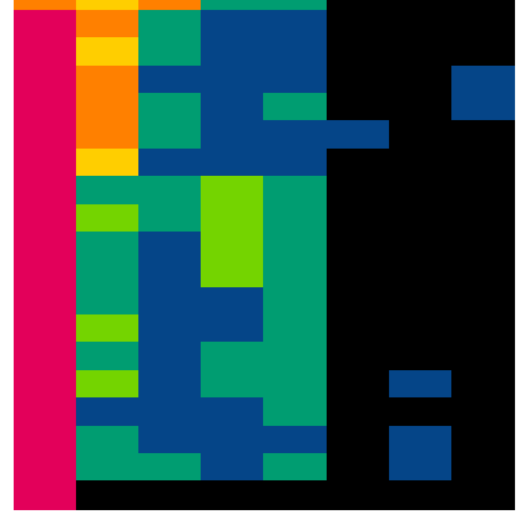

Supplemental Figure 2: (A) Weighted UniFrac tree of 16S rRNA pyrosequences spanning the V5-V7 hypervariable regions derived from the different TIM-2 samples (obese (0) \& lean (L), cassava [red], inulin [blue] and standard [black], time zero (t0) and time $72 \mathrm{~h}(\mathrm{t} 72 \mathrm{~h})$. No1-No3: replicate samples. Scale bars indicate distance between the samples in UniFrac units. (B) The relative abundance of bacterial phyla observed in these data sets is represented in a heatmap.

A

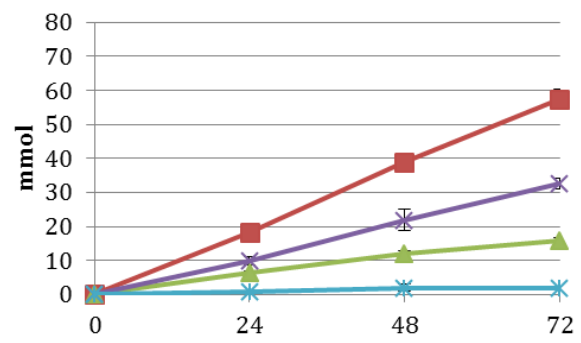

B

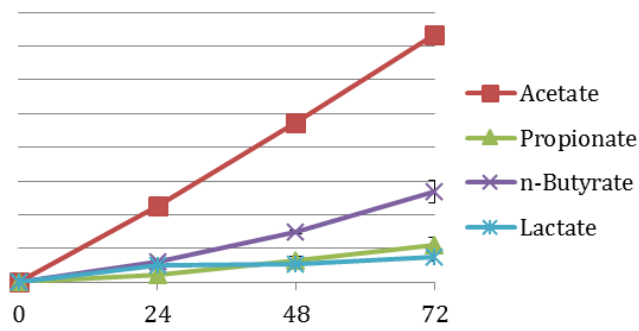

Supplemental Figure 3: Kinetics of average production of SCFA and lactate. Cumulative average production ( \pm range) of SCFA and lactate (mmol) over time during the 3-day experimental period upon feeding cassava at $7.5 \mathrm{~g} /$ day in lean $(\mathrm{A})$ and obese microbiotas (B). 
Genera

Bifidobacterium

Parabacteroides

Prevotella

Bacteroides

Enterococcus

Lactobacillus

Clostridium sensu stricto

Anaerostipes

Blautia

Clostridium XlVa

Coprococcus

Dorea

Lachnospiracea incertae sedis

Roseburia

Clostridium XI

Acetivibrio

Clostridium IV

Faecalibacterium

Subdoligranulum

Dialister

Catenibacterium

Erysipelotrichaceae incertae sedis

* Lactobacillus is shown here to emphasize that it did not change with any treatment

Supplemental Figure 4: Heatmap. Changes in microbiota composition in the lean and obese samples over the 72 hour period. Data are expressed as fold increased (green) or decrease (red) compared to standard. Intensity of the colour signifies degree of increase or decrease.
Cassava lean Inulin lean Cassava obese Inulin obese
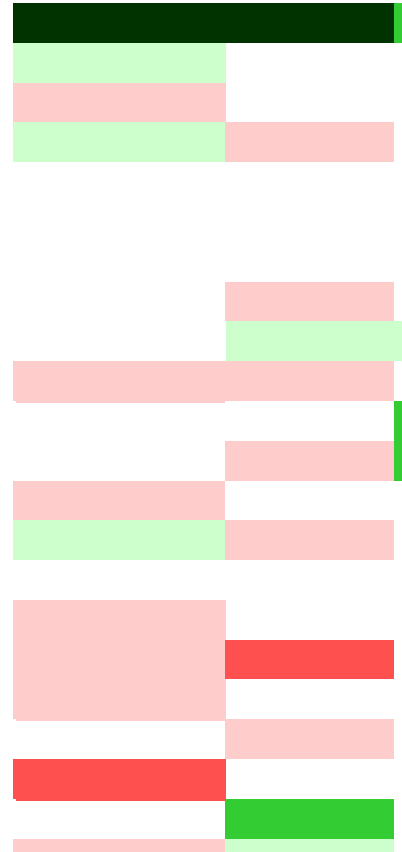

$>100$

$>10-100$

$>2-10$

$<2-10$

$<10-100$ 
Chapter 3 


\section{Chapter 4}

Lean and obese microbiota: differences in in vitro fermentation of by-products from Brazilian food industry

Bussolo de Souza, C., Saad, S., Venema, K. 


\section{Abstract}

Currently the consumption of dietary fibres is lower than recommended, leading to health problems, like obesity. Gut microbiota is being suggested as factor that contributes to obesity and it can be modulated through diet, especially dietary fibres. Its modulation in favour of the host might be a way to revert the scenario of obesity. Food by-products contain many important nutrients, including fibres. These by-products are mostly underused and their inappropriate disposal in the environment contributes to pollution. The aim of the present study was to investigate the potential prebiotic effects of food byproducts (cassava bagasse, orange bagasse and passion fruit peel) using an in vitro model that simulates the proximal colon, and to assess possible differences in fermentation when using a microbiota from lean or obese people. Fermentation of the by-products was compared to a standard medium (control) and the prebiotic inulin. The results revealed that the effects of the byproducts on the dynamics of the gut microbiota differed according to the type of microbiota - lean or obese, as well as the type of by-product used. Principal Coordinate Analysis (PCoA) of the microbiota showed evidence of a clear separate clustering of lean and obese microbiotas before the addition of test compounds, which disappeared at $\mathrm{t} 72 \mathrm{~h}$, and instead, distinct clusters due to primary composition of the substrates (starch, fructan and pectin) were present. This is evidence that the test compounds drove the obese microbiota to a healthier profile, more similar to that of the lean microbiota. Cassava bagasses enriched the beneficial genus Bifidobacterium. The production of total SCFA by cassava bagasses by the obese microbiota was higher than control and inulin (e.g. cassava bagasse 2 (CB2) generated $150.9 \mathrm{mmol}$, while this was 100 $\mathrm{mmol}$ and $108.9 \mathrm{mmol}$, for control and inulin respectively). Orange bagasses stimulated the growth of the genera Collinsella and Catenibacterium. Passion fruit peels were poorly fermented, generating smaller amounts of SCFA (e.g. passion fruit 2 (PFP2) resulted in the production of on average $77.8 \mathrm{mmol}$, either in lean or obese microbiotas) than control $(139.8 \mathrm{mmol}$ in the lean and $100 \mathrm{mmol}$ in the obese microbiotas) and inulin (137.2 $\mathrm{mmol}$ in the lean and $108.9 \mathrm{mmol}$ in the obese microbiotas). They also generated more BCFA (e.g. PFP1 resulted in the production of $5.2 \mathrm{mmol}$, while on inulin $0.2 \mathrm{mmol}$ was produced in the lean microbiota). Passion fruit peels fermentation resulted in a microbiota with the highest diversity and evenness, a positive trait regarding host health. In conclusion, the use of food by-products could be an important step to tackle obesity and decrease the waste of valuable food material and consequently environmental pollution. They are inexpensive and a noninvasive way to be used as a dietary intervention to improve health. 


\section{Introduction}

The problem of overweight and obesity (body mass index (BMI) $25-29.9 \mathrm{~kg} / \mathrm{m}^{2}$ and $\geq 30 \mathrm{~kg} / \mathrm{m}^{2}$, respectively) is reaching an incredible dimension worldwide, affecting half a billion adults (1). A BMI above the normal range increases the risk for several diseases, such as cardiovascular disease and different types of cancer (1). The gut microbiota is emerging as a possible tool to be used in this battle against obesity. Since it was discovered as an environmental factor that contributes to obesity about two decades ago (2), the gut microbiota is being subject of several investigations that try to reveal this complex mechanism between host, gut microbiota and obesity (3-5). In this context, host diet has a direct influence on microbiota composition (6), and is considered an important way to modulate the gut microbiota. Indigestible products that reach the colon are the main substrate for the gut microbiota, and among them, dietary fibres constitute an important source of energy for these microorganisms (7).

Nowadays, however, the food industry is producing more and more ultraprocessed foods by which several components from the raw material are removed and discarded, generating by-products (such as fruit and vegetable peels). These by-products are mostly underused and their inappropriate disposal in the environment contributes to pollution (8). Additionally, food industry loses part of the raw material that might have important nutritional value, such as fibre (9). At the same time, the consumption of ultra-processed foods that lack important nutrients, like fibres, but instead are filled with high energy ingredients (simple sugars/fats), is contributing to the increased incidence of diseases related with bad eating habits, especially obesity $(10,11)$. Fortunately, this problem is raising a debate about the use of by-products, as for instance fibre source, and the issue is gaining more importance and attention (8). A proper utilization of these by-products, such as to incorporate them back into food products, for example as functional foods, could represent one step to decrease their wastage and increase fiber intake. This interconnected scenario of lack of fibre consumption, modulation of the gut microbiota through ingestion of fibres and the use of fibre-rich by-products from the food industry seems durable with regards to a favourable human health and environmentally friendly reuse of wasted food. However, before incorporating these by-products into food, it is necessary to characterized them and to study their possible health effects. We have previously characterized the by-products used here from the Brazilian food industry - cassava bagasse, orange bagasse and passion fruit peel - regarding their chemical composition and digestibility (12). Interestingly, results showed differences in composition 
of the same by-product from different suppliers, which can have an influence on the health effects when consuming these fibres.

The aim of the present study was to investigate the potential prebiotic effects of these food by-products using an in vitro model that closely simulates the proximal colon (TNO intestinal model (TIM-2)) and assess possible differences in fermentation when using a microbiota from lean or obese people.

\section{Material and methods}

\section{Test compounds}

In total, 8 test compounds (plus 2 controls) were tested for the fermentation experiments. Three different cassava bagasses were tested, CB1and CB2 were from the same supplier but different batches (Grazimara, Biguaçu, Brazil), CB3 was from Lorenz (Indaial, Brazil). Two samples of orange bagasses were tested, both in the form of powder - OB1 from Chá\&Cia (Jacareí, Brazil), OB2 from a store with bulk sale in the city centre of Florianópolis. Three passion fruit peels were tested, all in the form of powder - PFP1 from Chá\&Cia, PFP2 from Phytomare (Governador Celso Ramos, Brazil) and PFP3 from the same store as OB2 (Florianópolis). According to the manufacturers, only the peels were used, seeds were disposed. Inulin from chicory root was provided by its manufacturer (Orafti, Amsterdam, the Netherlands) and it was used as positive control (purity $\geq 90 \%$, degree of polymerization $>10$ ). Simulated ileal effluent medium (SIEM) was used as control and is described in the following section. More detailed information about the test compounds was described elsewhere (12), but is summarized in Table 1. 


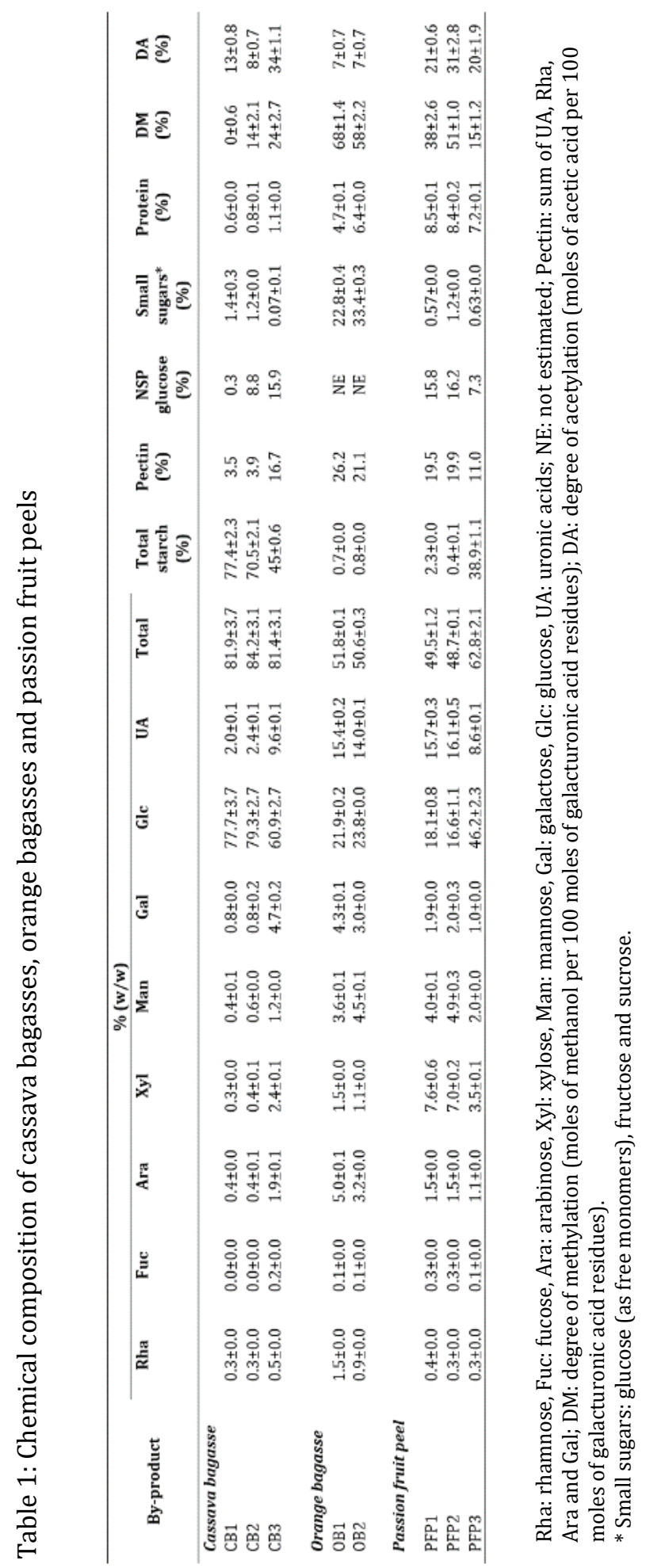




\section{In vitro fermentation}

\section{Dynamic in vitro model of the colon (TIM-2)}

TIM-2 is a validated, dynamic in vitro model that closely mimics the proximal colon, extensively described by Minekus et al. (13) (Figure 1). Briefly, this computer-controlled system simulates several features from the human large intestine, such as temperature (kept at $37{ }^{\circ} \mathrm{C}$ ), pH (kept at 5.8 - constantly checked using a $\mathrm{pH}$ sensor and corrected with secretion of sodium hydroxide $(\mathrm{NaOH})$ ), anaerobic environment (by constant flushing with gaseous nitrogen).

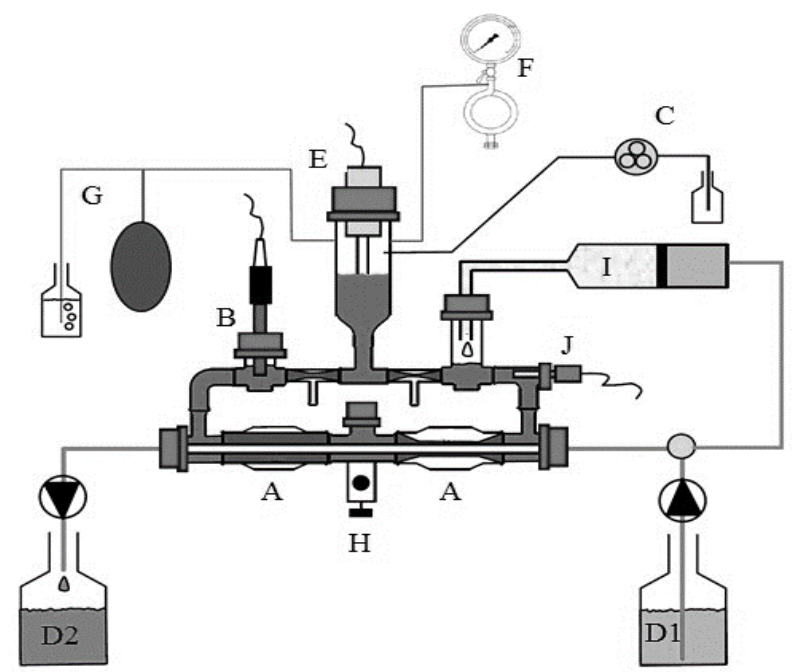

Figure 1: Schematic representation of the TIM-2 system. A) peristaltic compartments with a dialysis membrane inside, $\mathrm{B}$ ) $\mathrm{pH}$ sensor, $\mathrm{C}$ ) $\mathrm{NaOH}$ secretion, D) dialysate system (D1=dialysate in, D2=dialysate out), E) level sensor, F), gaseous $\mathrm{N}_{2}$ inlet, $\mathrm{G}$ ) gas outlet, H) sampling port, I) feeding syringe with test compound, J) temperature sensor. Adapted from (14).

Water and fermentation products are removed through a dialysis system. This prevents accumulation of microbial metabolites which would otherwise lead to inhibition or even death of the microbiota. Mixing and transport of intestinal contents is achieved through the simulation of peristaltic movements. It was previously demonstrated that TIM-2 allows for the growth of a highly active and dense microbiota, comparable to that found in the human proximal colon (15).

\section{Origin of the microbiotas}

The microbiotas used for the TIM- 2 experiments consisted of active pooled faecal material from $i$ ) 8 healthy lean volunteers (body mass index (BMI) $=20 \pm$ $1.48 \mathrm{~kg} / \mathrm{m}^{2}$, male: $\mathrm{n}=4$, female: $\mathrm{n}=4$, age range $25-42$ years) and referred to as 
"lean microbiota", or ii) 7 obese but otherwise healthy volunteers (BMI=32 $\pm 1.17 \mathrm{~kg} / \mathrm{m}^{2}$, male: $\mathrm{n}=3$, female: $\mathrm{n}=4$, age range $29-68$ years) denoted as "obese microbiota". Lean volunteers were recruited from TNO (Zeist, the Netherlands) and obese volunteers from Maastricht University Medical Centre (Maastricht, the Netherlands). Volunteers received oral and written explanation about the study and how to proceed with faeces collection. Prior to their participation, each person provided an informed consent. The exclusion criteria for both groups included the use of antibiotics, probiotics, prebiotics and laxatives in the 3 months preceding donation. Faecal samples were self-collected: volunteers were instructed to defecate in a bag and immediately place it into a gastight plastic jar containing anaerobic packs (AnaeroGen ${ }^{\mathrm{TM}}$, Oxoid, Cambridge, UK). An aliquot of each individual donation was collected as previously described (16) and used for analyses of microbiota composition and metabolites (SCFA and BCFA). Faeces were mixed in an anaerobic cabinet as described by Aguirre et al. (17). The faecal material was subsequently aliquoted, snap-frozen in liquid nitrogen and stored at $-80^{\circ} \mathrm{C}$. It was previously shown that the standardization of the microbiota (pooling) does result in a representative microbiota in terms of composition and activity (18), and is similar to fresh faecal sample (19).

\section{Simulated ileal effluent medium (SIEM) and dialysate}

SIEM was used as standard feeding and simulates the material reaching the colon. It is basically composed of indigestible carbohydrates (pectin, xylan, arabinogalactan, amylopectin and starch), protein, vitamins, Tween 80 and bile (17). The $\mathrm{pH}$ was adjusted to 5.8 to simulate the $\mathrm{pH}$ from the proximal colon. Dialysate contained (per litre): $2.5 \mathrm{~g} \mathrm{~K} \mathrm{~K}_{2} \mathrm{HPO}_{4} .3 \mathrm{H}_{2} \mathrm{O}, 4.5 \mathrm{~g} \mathrm{NaCl}, 0.005 \mathrm{~g}$ $\mathrm{FeSO}_{4} .7 \mathrm{H}_{2} \mathrm{O}, 0.5 \mathrm{~g} \mathrm{MgSO}_{4} .7 \mathrm{H}_{2} \mathrm{O}, 0.45 \mathrm{~g} \mathrm{CaCl} 2.2 \mathrm{H}_{2} \mathrm{O}, 0.05 \mathrm{~g}$ bile and $0.4 \mathrm{~g}$ cysteine $\cdot \mathrm{HCl}$, plus $1 \mathrm{~mL}$ of the vitamin mixture; pH 5.8 (17). All medium components were acquired at Tritium Microbiology (Eindhoven, the Netherlands).

\section{Experimental protocol and study design}

Before the addition of the standardized microbiota to the system, TIM-2 was flushed with gaseous nitrogen for 3 hours to create a complete anaerobic environment. Afterwards, the system was inoculated with approximately $30 \mathrm{~mL}$ of the standardized microbiota and $90 \mathrm{~mL}$ of dialysate (described above). The microbiota was fed with SIEM $(2.5 \mathrm{~mL} /$ hour $)$ and adapted to the system for 16 hours. After the adaptation period, the feeding system was stopped and a 2 hours starvation period allowed the microbiota to ferment all remaining carbohydrates present in the model. Subsequently, samples were collected at 
time zero ( $\mathrm{t} 0 \mathrm{~h}$ ) after which the test compounds were added. From this point onwards, a 3-day experimental period started, and the microbiota received the standard feeding (SIEM, referred to from now on as control), or this medium but without the carbohydrate source, which was substituted with the test compounds - inulin, cassava bagasse, orange bagasse or passion fruit peel. SIEM contained $7.5 \mathrm{~g} /$ day of carbohydrates and the same amount of test compounds was added. Samples of lumen and spent dialysate (dialysate out) were taken at time 0 hour ( $\mathrm{t} 0 \mathrm{~h}$ ), time 24 hours ( $\mathrm{t} 24 \mathrm{~h})$, time 48 hours ( $\mathrm{t} 48 \mathrm{~h}$ ) and time 72 hours (t72 h). Four TIM-2 units were run in parallel. The experimental set up can be viewed in Figure 2.
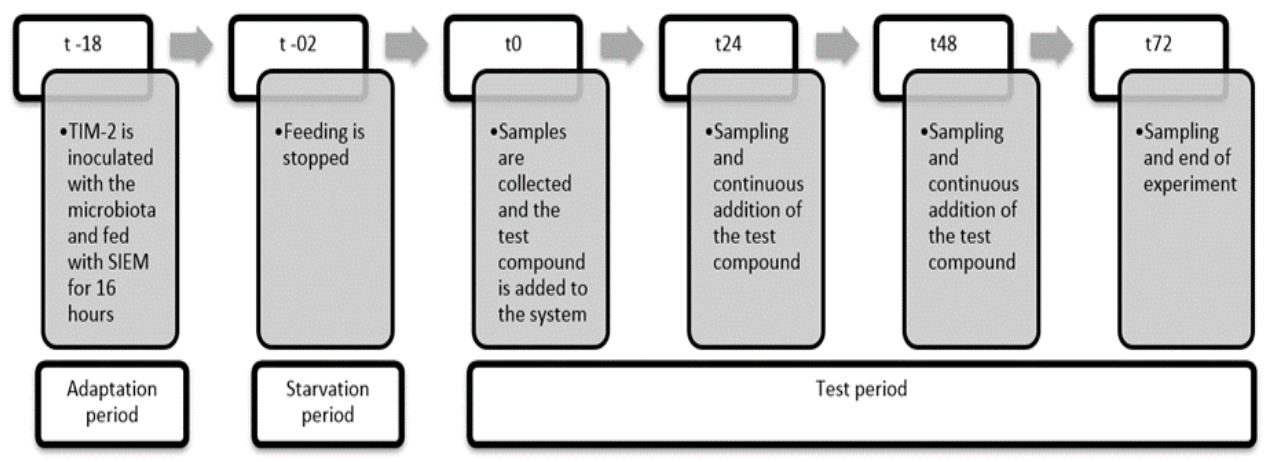

Figure 2: Study design with timeline for TIM-2 experiments.

\section{Analyses}

\section{SCFA, BCFA and organic acids quantification in lumen and dialysate out samples}

Lumen and dialysate out samples from TIM-2 were analysed by Brightlabs (Venlo, the Netherlands) to quantify SCFA (acetate, propionate and butyrate), BCFA (iso-butyrate and iso-valerate), as well as organic acids (valerate, caproate, lactate, succinate and formate). Samples were centrifuged at 14.000 rpm for 10 minutes, and the clear supernatant was filtered through a $0.45 \mu \mathrm{m}$ PFTE filter and diluted with mobile phase (lumen 1:5 and dialysate out 1:2). Ion exclusion chromatography (IEC) was applied in a 883 Ion Chromatograph (IC; Metrohm, Switzerland), using a Transgenomic IC Sep ICE-ION-300 column (30 cm length, $7.8 \mathrm{~mm}$ diameter and $7 \mu \mathrm{m}$ particles) and a MetroSep RP2 Guard. The mobile phase consisted of $1.5 \mathrm{mM}$ aqueous sulphuric acid. A column flow of $0.4 \mathrm{ml} / \mathrm{min}$ was used. The temperature of the column was $65^{\circ} \mathrm{C}$. The organic acids were detected using suppressed conductivity detection. Ten microliters were loaded on the column by an autosampler 730 (Metrohm). Molecules were 
eluted according to their pKa. To show cumulative production upon addition of substrates, the amount of metabolites was artificially put to zero at t0 $\mathrm{h}$.

\section{Energy extraction}

Energy extraction in the form of SCFA was calculated using the following values for acetate, propionate, butyrate and lactate, respectively: 209, 367, 524 and $326 \mathrm{kcal} / \mathrm{mol}$.

\section{Microbiota composition}

Genomic DNA isolation from TIM-2 samples (lumen, t0 $\mathrm{h}$ and t72 h) was performed using standard molecular biology kits from ZYMO (ZYMO Research, CO., CA, USA) by Baseclear (Leiden, the Netherlands). PCR amplification of the 16S rRNA gene (V3-V4 region), barcoding and library preparation were also performed by Baseclear. Short paired-end sequence reads were generated using the Illumina MiSeq system and converted into FASTQ files using the BCL2FASTQ pipeline version 1.8.3. Quality trimming was applied based on Phred quality scores.

\section{Statistics}

Experiments were performed in duplicate $(n=2)$ per test compound and per microbiota (lean or obese), except for control ( $n=3$ with lean microbiota and $\mathrm{n}=4$ with obese microbiota) and inulin $(\mathrm{n}=3)$.

Microbial analyses were performed with Quantitative Insight Into Microbial Ecology (QIIME) software package (version 1.9.0) (20). The sequences were classified using Greengenes (version 13.8) as a reference 16S rRNA gene database. R (version 3.1.3) was used in RStudio for statistical analyses (21). Differences in multiple categories were tested with Kruskal-Wallis, with pvalues $p<0.05$ set to be significantly different. Dunn post hoc test was utilized to find differences between groups. Spearman correlation was calculated between the relative abundance of OTUs and continuous variables. Multiple comparisons were corrected using the false discovery rate (FDR), and q-values (adjusted pvalues) were considered significantly different at $<0.05$. Changes in microbial composition were assessed through the difference of their initial relative abundance and final abundance ( $\mathrm{t} 72 \mathrm{~h}-\mathrm{t} 0 \mathrm{~h})$, and compared to control. Linear discriminant analysis effect size (LEfSe) (22) was used to find biomarkers between groups using relative abundances from the OTU tables generated in QIIME. 


\section{Results and discussion}

\section{Characteristics of test compounds}

The chemical compositions of the eight test compounds used in TIM-2 fermentations are shown in Table 1, but they have been reported previously in detail elsewhere (12).

Briefly, regarding cassava bagasses, the two samples from the same origin but different batches were similar between each other (CB1 and CB2) but different from the third test compound that was supplied by another company (CB3). The differences are many, including the percentage of each monosaccharide, the total percentage of starch, in which CB3 presented a lower amount than CB1 and CB2 (77.4\% in CB1, 70.5\% in CB2 and 45\% in CB3), as well as the amount of pectin and non-starch polysaccharides (NSP), present in higher amount in CB3 (Table 1). Degree of methylation (DM) and acetylation (DA) were also higher for CB3. The orange bagasses tested were similar in their chemical composition (Table 1), even though they were supplied by different companies. Small variations were found regarding the amount of pectin present (26.2\% in OB1 and $21.1 \%$ in OB2), small sugars $(22.8 \%$ in OB1 and $33.4 \%$ in OB2), protein and DM. Two passion fruit peels were similar between each other (PFP1 and PFP2) but differed from the third sample (PFP3). The main differences were related to the amount of glucose and starch (higher for PFP3), pectin, NSP and DM (lower for PFP3) (Table 1).

\section{Effects of by-products on bacterial metabolic activity}

Studies are suggesting that many of the effects of the gut microbiota on host metabolic processes, especially on energy homeostasis, can be attributed to bacterial fermentation end-products - SCFA, BCFA and organic acids (23). The Bacteroidetes phylum contains species that produce mainly acetate and propionate, while species that belong to Firmicutes are responsible for the production of mostly butyrate.

\section{Total SCFA}

The amount of SCFA produced by the lean and obese microbiota was dependent on the by-product tested, and demonstrated that not always the obese microbiota generated more SCFA than the lean microbiota (Figure 3 and Supplemental Figure 1). The same was the case for energy extraction in the form of SCFA that was also dependent of the test compound, and not always related to the type of microbiota used for fermentation, as expected (Supplemental Figure 2). For 5 out of 10 substrates the obese microbiota was 
able to produce more total SCFA than the lean, although when comparing the total amount of SCFA produced by the lean and obese microbiotas (from all test compounds), the differences were not significant ( $p>0.05)$. Similar results are described in the literature. In vitro fermentation of diverse types of fibres showed that production of SCFA and consequently energy extraction was dependent of the test compound used, not the type of inoculum (lean or obese) (24), which is in accordance with our results.

A

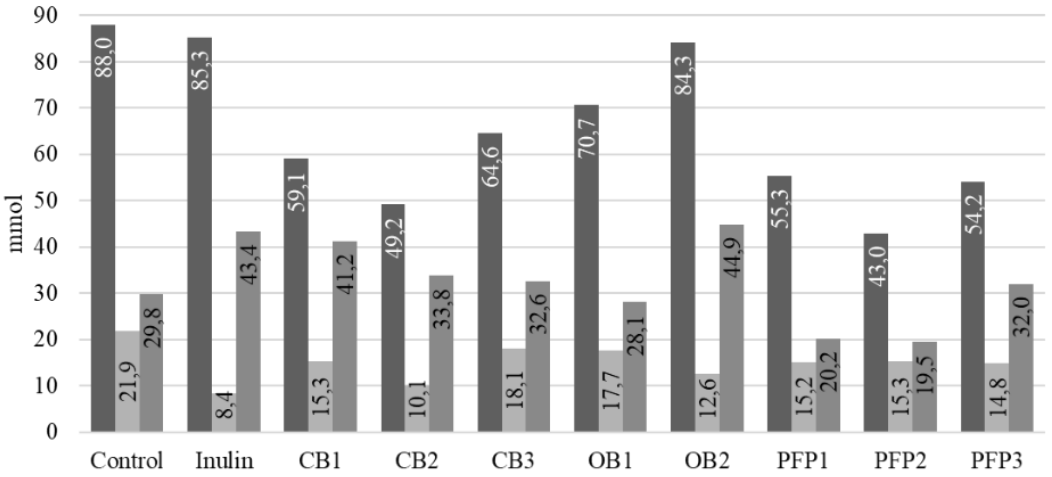

B

Acetate

= Propionate

=Butyrate

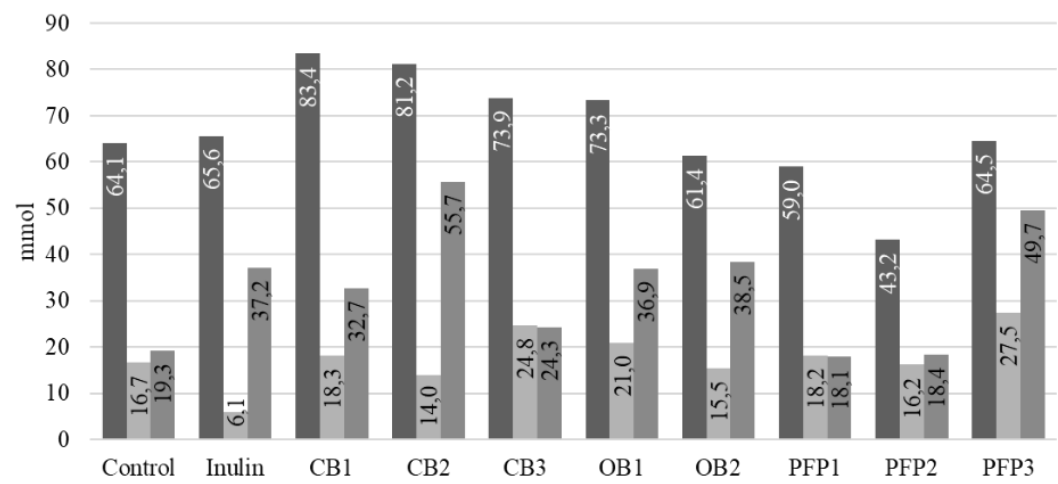

Figure 3: Cumulative production of SCFA (acetate, propionate and butyrate) by the lean (A) and obese (B) microbiotas at $\mathrm{t} 72 \mathrm{~h}$ with different test compounds.

The role of SCFA in obesity - either protective or causative, remains to be elucidated. However, it is worth noting that when detrimental aspects are related to SCFA, this is generally present when there is a dysbiosis - related to a certain disease or inappropriate life style. In turn, these aspects affect normal microbiota functioning - compositionally and metabolically speaking (25). Under "normal" conditions (health state), it has to be acknowledged that SCFA are essential in maintaining the equilibrium in host health through regulation and participation in several metabolic pathways. 
Production of total SCFA by fermentation of cassava bagasses yielded comparable amounts than control and inulin, demonstrating that the microbiota was able to use this substrate as energy source. Although CB3 had a distinct chemical composition compared to CB1 and CB2, in the lean microbiota the fermentation profiles were similar. In the obese microbiota, nonetheless, CB3 fermentation generated lower amounts of butyrate than CB1 and CB2.

Fermentation of orange bagasses by the obese microbiota produced more total SCFA than control and inulin, showing the potential of this by-product to be used as substrate by the gut microbiota. In the lean microbiota, fermentation of $\mathrm{OB} 2$ was very effective in producing butyrate, as well as acetate.

PFP1 and PFP2 were the substrates that compared to control and inulin, had the poorest fermentation, yielding smaller amounts of SCFA. PFP3, which contained higher amounts of starch, yielded comparable amounts of total SCFA than control and inulin.

\section{Acetate}

Apart from the two controls, SIEM and inulin, fermentation of OB1 in the lean microbiota had the highest cumulative production of acetate $(84.3 \mathrm{mmol})$ (Figure 3 and Supplemental Figure 1). A similar cumulative production of acetate was found upon CB1 and CB2 fermentation in the obese microbiota ( $83.4 \mathrm{mmol}$ and $81.2 \mathrm{mmol}$, respectively). From to $\mathrm{h}$ to $\mathrm{t} 24 \mathrm{~h}$, either in lean or obese microbiota, all test compounds increased the proportion of acetate (Supplemental Figure 3). After t24 h, however, an inverse effect occurred, and the proportion of acetate decreased over time. On average, cumulative production of acetate by the lean and obese microbiotas was similar. The role of acetate in obesity is not completely understood, and studies in mice and humans are inconsistent. Acetate is absorbed by intestinal cells and it is mainly found in peripheral circulation, thus capable of reaching a plethora of tissues (26). An investigation performed with obese individuals who consumed vinegar for 12 weeks resulted in lower body weight, BMI and visceral fat than the control group (27). Similar results were found in a recent study with mice where the goal was to evaluate the effects of synthetic acetic acid vinegar and Nipa vinegar on high-fat diet-induced obese mice (28). Results showed that both vinegars were effective in reducing food intake and body weight (28). Another study that demonstrates the positive effect of acetate related to obesity was performed with overweight and obese men which received infusions of acetate in the distal and proximal colon (29). It was found that distal infusions of acetate, but not proximal, increased fasting fat oxidation and fasting peptide YY (PYY), when compared to the placebo group (29). In a study by Perry et al 
(30), nevertheless, increased acetate production by an altered microbiota was found to rise ghrelin secretion, causing hyperphagia and obesity in rats fed with high fat diet.

\section{Propionate}

In the lean microbiota, CB3 and OB1 were the substrates that had the highest cumulative production of propionate $(18.1 \mathrm{mmol}$ and $17.7 \mathrm{mmol}$, respectively) (Figure 3 and Supplemental Figure 1). Apart from control and inulin the fermentation of which caused an increase in the proportion of propionate over time, all other test compounds had a stable proportion of propionate after $\mathrm{t} 24 \mathrm{~h}$ (Supplemental Figure 3). In the obese microbiota CB3 and OB1 were also responsible for a higher cumulative production of propionate $(24.8 \mathrm{mmol})$. PFP3, however, showed an even higher amount of propionate $-27.5 \mathrm{mmol}$ (Figure 3 and Supplemental Figure 3). Looking at the ratio of each SCFA, although the proportion of propionate was higher in the obese microbiota compared to the lean microbiota, it remained constant over time (Supplemental Figure 3). Propionate is produced by colonic microbiota fermentation of polyand oligosaccharides, long chain fatty acids, protein, peptides and glycoprotein $(31,32)$. It is metabolized in the liver, used as substrate for gluconeogenesis, and therefore converted into glucose (26). Increased gluconeogenesis via propionate was suggested to decrease food intake. It was proposed that increased hepatic production and storage of glucose would be sensed by the central nervous system (CNS), which in turn would regulate eating behaviour (26). A study with mice revealed that propionate was already converted to glucose in the intestine, through intestinal gluconeogenesis (IGN), decreasing body weight and adiposity (33). The mechanism proposed is that IGN promoted the release of glucose in the portal vein, resulting in decreased hepatic glucose production and increased satiety and energy expenditure via the CNS $(23,33)$. Another mechanism by which propionate was found to improve satiety is via the activation of free fatty acid receptors (FFAR). Propionate was found to be a ligand of FFAR3 and FFAR2 (34). FFAR triggers the release of the satiety hormones glucagon-like peptide-1 (GLP-1) and PYY by L-cells (34). PYY inhibits gastric emptying and food intake, whereas GLP-1 stimulates insulin secretion $(35,36)$. Increasing transit time of ingested food through the gastrointestinal tract prolongs the stimulation of mechanic/chemical receptors responsible for signalling satiety after food intake (26). 


\section{Butyrate}

Fermentation of OB2 by the lean microbiota produced the highest cumulative amount of butyrate $(44.9 \mathrm{mmol})$, slightly higher amounts than our positive control - inulin (43.4 mmol) (Figure 3 and Supplemental Figure 1). CB1, however, presented the highest proportion of butyrate at t72 h (38.4\%) (Supplemental Figure 3). An in vitro study with acerola by-product demonstrated the beneficial effects in bacterial metabolism when adding this compound in combination with the probiotic Bifidobacterium longum BB-46 (37). During the treatment period, a significant increase in the production of SCFA, especially butyrate was observed. Acerola by-product was rich in fibre $(\sim 56 \%)$, presented high content of phenolic compounds and antioxidant activity (37). Unfortunately, the type of fibres was not analysed in this research, making it difficult to compare between studies. A synergetic effect of the different components (fibres and polyphenols) present in acerola by-product might be responsible for the positive outcome encountered. This synergetic effect may also occur with orange bagasses, since analysis also demonstrated that this by-product is rich in bioactive compounds, such as polyphenols (38). In the obese microbiota, CB2 was the substrate that generated highest cumulative amounts of butyrate at $\mathrm{t} 72 \mathrm{~h}$, followed by PFP3 and OB2 (Figure 3 and Supplemental Figure 1). Fermentation of CB2 by the obese microbiota also had the highest proportion of butyrate at $\mathrm{t} 72 \mathrm{~h}(36.9 \%)$. Citrus pectin has been found to stimulate the production of butyrate in an in vitro model inoculated with microbiota from obese individuals (39), which corroborates with the results from our study. Butyrate is by far the most well studied SCFA, and many investigations have shown its beneficial aspects either at the intestinal level, such as anticarcinogenic agent and its role in ion absorption, as well as at the extraintestinal level, like improving hypercholesterolemia and acting as ammonia scavenger (40). Butyrate constitutes the main source of energy for colonocytes, supplying circa $60-70 \%$ of the required energy (23), and thus is rapidly used by the epithelium (41). It has, therefore, a fundamental function in maintaining colonic mucosal health. With regard to obesity, butyrate has an important role in keeping the integrity of the intestinal barrier by acting on the mucosal layer and tight junctions. Studies with cell lines demonstrated that butyrate was responsible for stimulating the production of mucin MUC2 $(42,43)$. Similar results, nonetheless, could not be replicated in a human study. A clinical trial with healthy individuals and ulcerative colitis patients showed that intervention with butyrate did not modulate the expression of MUC2 in both healthy and ulcerative colitis volunteers (44). More human studies are necessary to confirm the effects of butyrate in the production of intestinal 
mucin. Other studies with cell lines showed that butyrate enhanced intestinal barrier by regulating the assembly and stimulating the synthesis of tight junctions $(45,46)$. The importance of intestinal barrier regarding obesity is because an enhanced protection against translocation of LPS and pathogens, for instance, will avoid the activation of pro-inflammatory signalling cascade, which induce metabolic diseases characterized by low-grade inflammation (such as in obesity), as well as insulin resistance (described in more detail in the following section).

\section{Branched-chain fatty acids and organic acids}

BCFA production was much lower than SCFA. In the lean microbiota, PFP1 fermentation produced the highest amount of iso-butyrate $(1.92 \mathrm{mmol})$, whilst in the obese microbiota PFP3 fermentation was responsible for the highest amount of iso-valerate $(3.35 \mathrm{mmol})$. Overall, passion fruit peels were the substrates that led to the highest cumulative amounts of BCFA, both in experiments with the lean and obese microbiotas (Table 2).

Table 2: Cumulative production of BCFA (iso-butyrate and iso-valerate) by the lean and obese microbiotas after $72 \mathrm{~h}$ fermentation with the different test compounds.

\begin{tabular}{ccccccc}
\hline & \multicolumn{2}{c}{$\boldsymbol{i}$-butyrate } & \multicolumn{2}{c}{$\boldsymbol{i}$-valerate } & \multicolumn{2}{c}{ Total } \\
\cline { 2 - 7 } & Lean & Obese & Lean & Obese & Lean & Obese \\
\cline { 2 - 7 } Control & $0.2 \pm 0.2$ & $0.5 \pm 0.8$ & $0.4 \pm 0.1$ & $1.3 \pm 1.2$ & $0.6 \pm 0.3$ & $1.9 \pm 2.0$ \\
Inulin & $0.1 \pm 0.0$ & $0.0 \pm 0.0$ & $0.2 \pm 0.1$ & $0.0 \pm 0.0$ & $0.2 \pm 0.1$ & $0.0 \pm 0.0$ \\
CB1 & $0.7 \pm 0.1$ & $0.7 \pm 0.3$ & $2.1 \pm 0.2$ & $1.5 \pm 0.4$ & $2.7 \pm 0.1$ & $2.2 \pm 0.7$ \\
CB2 & $0.6 \pm 0.2$ & $0.3 \pm 0.0$ & $1.3 \pm 0.2$ & $0.7 \pm 0.0$ & $1.9 \pm 0.5$ & $1.0 \pm 0.1$ \\
CB3 & $1.7 \pm 0.1$ & $0.5 \pm 0.4$ & $1.5 \pm 0.1$ & $0.8 \pm 0.3$ & $3.2 \pm 0.0$ & $1.3 \pm 0.7$ \\
OB1 & $1.2 \pm 0.0$ & $0.2 \pm 0.1$ & $2.2 \pm 0.3$ & $1.2 \pm 0.3$ & $3.4 \pm 0.3$ & $1.4 \pm 0.5$ \\
OB2 & $0.9 \pm 0.1$ & $0.2 \pm 0.2$ & $2.1 \pm 0.0$ & $0.2 \pm 0.0$ & $2.9 \pm 0.1$ & $0.4 \pm 0.2$ \\
PFP1 & $1.9 \pm 0.2$ & $1.8 \pm 0.3$ & $3.3 \pm 0.3$ & $3.0 \pm 0.2$ & $5.2 \pm 0.5$ & $4.8 \pm 0.5$ \\
PFP2 & $1.4 \pm 0.3$ & $0.6 \pm 0.1$ & $2.3 \pm 0.4$ & $1.6 \pm 0.0$ & $3.8 \pm 0.7$ & $2.2 \pm 0.1$ \\
PFP3 & $1.7 \pm 0.1$ & $1.7 \pm 0.7$ & $2.9 \pm 0.4$ & $3.4 \pm 1.0$ & $4.6 \pm 0.5$ & $5.1 \pm 1.8$ \\
\hline
\end{tabular}

The passion fruit peels contained higher amounts of proteins, which may explain the production of BCFA, as these are the end-products of bacterial fermentation of proteins and peptides (47). They generated negligible amounts of lactate and succinate (Table 3), indicating that these by-products were not fermented at a high speed. Lactate and succinate are intermediate metabolites and their accumulation is a sign of fast fermentation (48). The effects of passion fruit peel flour on metabolic parameters, such as insulin sensitivity, body weight and gut hormones, were tested in rats fed a high fat diet (49). Results 
showed that rats that received supplementation of passion fruit peel flour in addition to high fat diet presented an increase in glucagon like-peptide 1 (GLP1) release, decreased adiposity and improved insulin sensitivity - amongst others (49). Bacterial community and production of SCFA were not measured in this study, nevertheless these effects are thought to be mediated by the release of SCFA from bacterial fermentation of pectin present in passion fruit peel flour.

Inulin was the substrate that yielded more organics acids, especially lactate and succinate (Table 3 ).

Table 3: Cumulative production of organic acids (succinate, lactate, formate and caproate) by the lean and obese microbiotas after $72 \mathrm{~h}$ fermentation with the different test compounds.

\begin{tabular}{|c|c|c|c|c|c|c|c|c|c|c|}
\hline \multirow[b]{3}{*}{ Control } & \multicolumn{2}{|c|}{ Succinate } & \multicolumn{2}{|c|}{ Lactate } & \multicolumn{2}{|c|}{ Formate } & \multicolumn{2}{|c|}{ Caproate } & \multicolumn{2}{|c|}{ TOTAL } \\
\hline & Lean & Obese & Lean & Obese & Lean & Obese & Lean & Obese & Lean & Obese \\
\hline & $2.1 \pm 0.5$ & $7.1 \pm 6.6$ & $0.6 \pm 0.0$ & $1.1 \pm 0.7$ & $4.5 \pm 0.7$ & $3.6 \pm 1.2$ & $0.0 \pm 0.0$ & $0.0 \pm 0.0$ & $7.2 \pm 1.1$ & $11.8 \pm 6.0$ \\
\hline Inulin & $7.2 \pm 4.4$ & $0.2 \pm 0.0$ & $7.6 \pm 2.0$ & $14.4 \pm 14.0$ & $5.1 \pm 1.1$ & $3.1 \pm 0.8$ & $0.0 \pm 0.0$ & $0.0 \pm 0.0$ & $19.9 \pm 5.5$ & $17.7 \pm 14.8$ \\
\hline CB1 & $0.8 \pm 0.2$ & $2.1 \pm 0.1$ & $0.2 \pm 0.0$ & $4.1 \pm 2.3$ & $3.9 \pm 0.4$ & $5.7 \pm 0.1$ & $0.2 \pm 0.1$ & $0.0 \pm 0.0$ & $5.2 \pm 0.8$ & $11.9 \pm 2.3$ \\
\hline CB2 & $2.9 \pm 0.7$ & $0.2 \pm 0.0$ & $0.4 \pm 0.4$ & $5.7 \pm 0.2$ & $6.2 \pm 0.0$ & $4.0 \pm 1.1$ & $0.2 \pm 0.1$ & $0.0 \pm 0.0$ & $9.8 \pm 1.2$ & $9.9 \pm 1.2$ \\
\hline CB3 & $0.9 \pm 0.5$ & $0.2 \pm 0.0$ & $0.2 \pm 0.1$ & $0.2 \pm 0.2$ & $2.9 \pm 0.4$ & $1.8 \pm 0.5$ & $0.3 \pm 0.2$ & $0.0 \pm 0.0$ & $4.3 \pm 1.4$ & $2.2 \pm 0.7$ \\
\hline OB1 & $0.6 \pm 0.1$ & $1.3 \pm 0.7$ & $0.0 \pm 0.0$ & $0.1 \pm 0.7$ & $0.2 \pm 0.0$ & $2.3 \pm 0.5$ & $1.2 \pm 0.8$ & $0.1 \pm 0.1$ & $2.0 \pm 0.9$ & $3.9 \pm 0.5$ \\
\hline OB2 & $3.1 \pm 0.7$ & $-0.1 \pm 0.1$ & $1.0 \pm 0.5$ & $0.0 \pm 0.1$ & $2.2 \pm 0.1$ & $1.0 \pm 0.1$ & $0.3 \pm 0.3$ & $0.2 \pm 0.2$ & $6.7 \pm 1.0$ & $1.1 \pm 0.3$ \\
\hline PFP1 & $0.7 \pm 0.0$ & $0.1 \pm 0.3$ & $-0.2 \pm 0.2$ & $0.1 \pm 0.2$ & $0.8 \pm 0.6$ & $1.1 \pm 0.9$ & $1.4 \pm 0.6$ & $0.0 \pm 0.1$ & $2.7 \pm 0.1$ & $1.2 \pm 1.3$ \\
\hline PFP2 & $0.3 \pm 0.1$ & $-0.2 \pm 0.0$ & $0.0 \pm 0.0$ & $-0.6 \pm 0.4$ & $1.6 \pm 0.8$ & $1.2 \pm 1.3$ & $0.4 \pm 0.1$ & $0.0 \pm 0.0$ & $2.3 \pm 0.9$ & $0.5 \pm 1.6$ \\
\hline PFP3 & $0.4 \pm 0.2$ & $0.2 \pm 0.1$ & $-0.1 \pm 0.1$ & $0.9 \pm 0.3$ & $1.4 \pm 1.0$ & $4.1 \pm 0.3$ & $0.6 \pm 0.2$ & $0.0 \pm 0.0$ & $2.3 \pm 1.5$ & $5.2 \pm 0.6$ \\
\hline
\end{tabular}

The role of these organic acids in health and disease is almost not known, but based on the concentrations observed, they should be studied. Elevated levels of plasma succinate was found to be associated with obesity and impaired glucose metabolism in Caucasian Spanish subjects (50). Interestingly, this was accompanied by the increased abundance of succinate-producing Prevotellaceae and Veillonellaceae and lower relative abundance of succinateconsuming Odoribacteraceae and Clostridaceae in obese subjects (50). The authors suggested that as a consequence of obesity, increased gut permeability and dysbiosis favoured higher amounts of succinate to reach plasma circulation in obese individuals.

\section{Changes in microbiota composition}

The results found in our study revealed that the effects of the by-products on the dynamics of the production of gut microbial metabolites differed according to the type of microbiota - lean or obese, as well as the type of by-product used. It was suspected that this was also the case for microbiota composition. 
Therefore, the composition of samples at $\mathrm{t} 0 \mathrm{~h}$ and $\mathrm{t} 72 \mathrm{~h}$ were analysed by sequencing the V3-V4 region of the $16 \mathrm{~S}$ rRNA gene.

Principal Coordinate Analysis ( $\mathrm{PCoA}$ ) showed a clear clustering of lean and obese microbiotas before the addition of test compounds (t0 h), in both weighted and unweighted UniFrac analyses (Figure $4 \mathrm{~A}$ and $4 \mathrm{~B}$ ). At $\mathrm{t} 72 \mathrm{~h}$, this clustering disappeared, and instead, distinct clusters by primary composition of the substrates (starch, fructan or pectin), and no longer distinction between lean and obese microbiota, was present (Figure 4C and 4D for weighted and unweighted UniFrac). This is evidence that the test compounds drove the obese microbiota to a healthier profile, that is more similar to that of the lean microbiota. The Shannon index showed that fermentation of PFP3, OB1 and PFP1 stimulated the growth of more bacterial species (abundance and evenness) (Supplemental Figure 4).
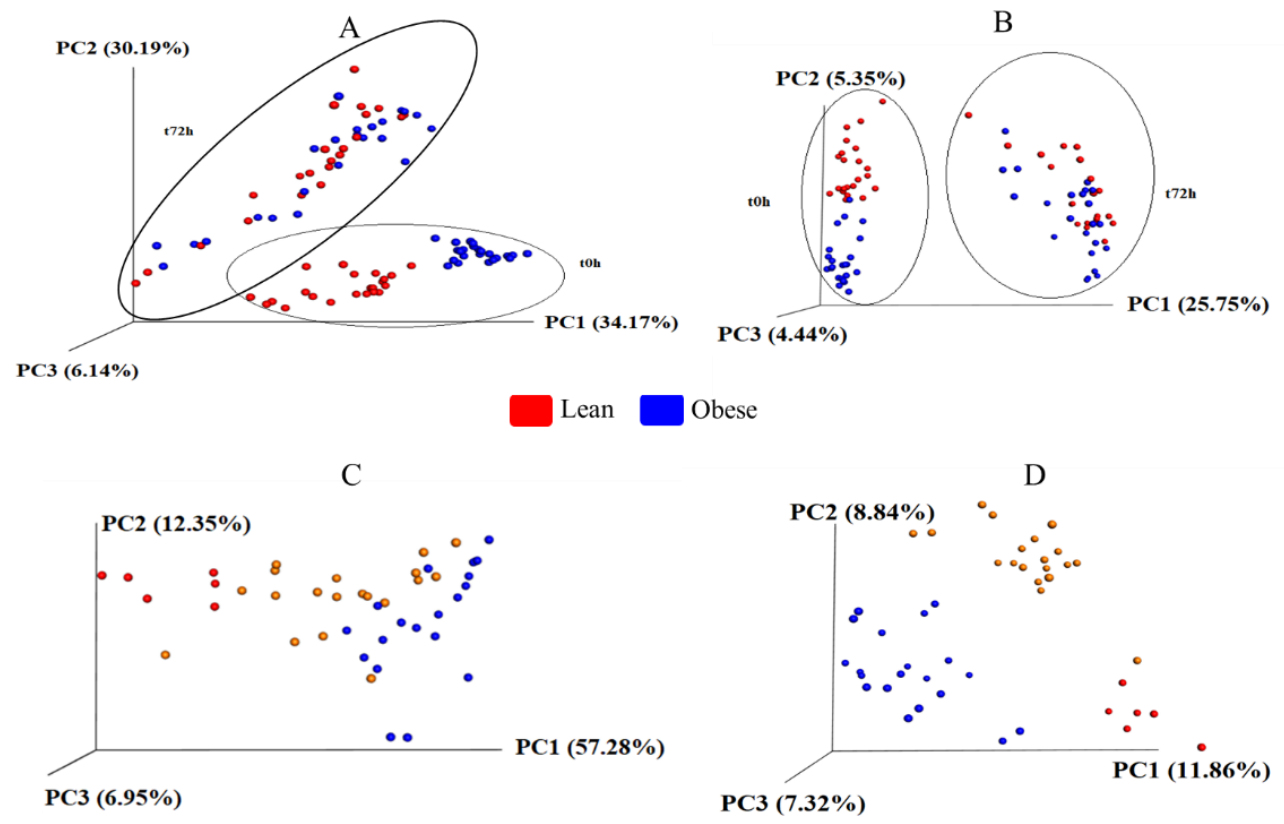

Obese

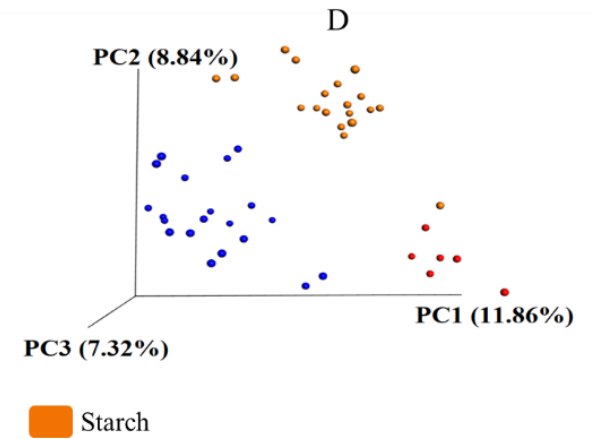

Figure 4: Principal Coordinate Analysis (PCoA) plots of the microbiota after 72 hours fermentation period in TIM-2. Samples are plotted on the first three Principal Coordinates (PC1, PC2 and PC3). A and B: Weighted and Unweighted (respectively) UniFrac showing 2 different clusters among lean and obese samples at toh, which disappeared after 72 hours fermentation period. C and D: Weighted and Unweighted (respectively) UniFrac showing that at $\mathrm{t} 72 \mathrm{~h}$, samples were clustered according to their primary substrate (fructan, pectin or starch).

The effects that fermentation of each test compound had on the microbial composition were assessed also through the difference of their initial relative 
abundance and final abundance ( $\mathrm{t} 72 \mathrm{~h}-\mathrm{t} 0 \mathrm{~h})$, and compared to their respective control (lean or obese). This will be discussed below for selected genera for which effects were observed.

\section{Bifidobacterium}

In our study, for the lean microbiota, all tested compounds (except inulin) caused a decrease of this genus when compared to the control (Figure 5A).
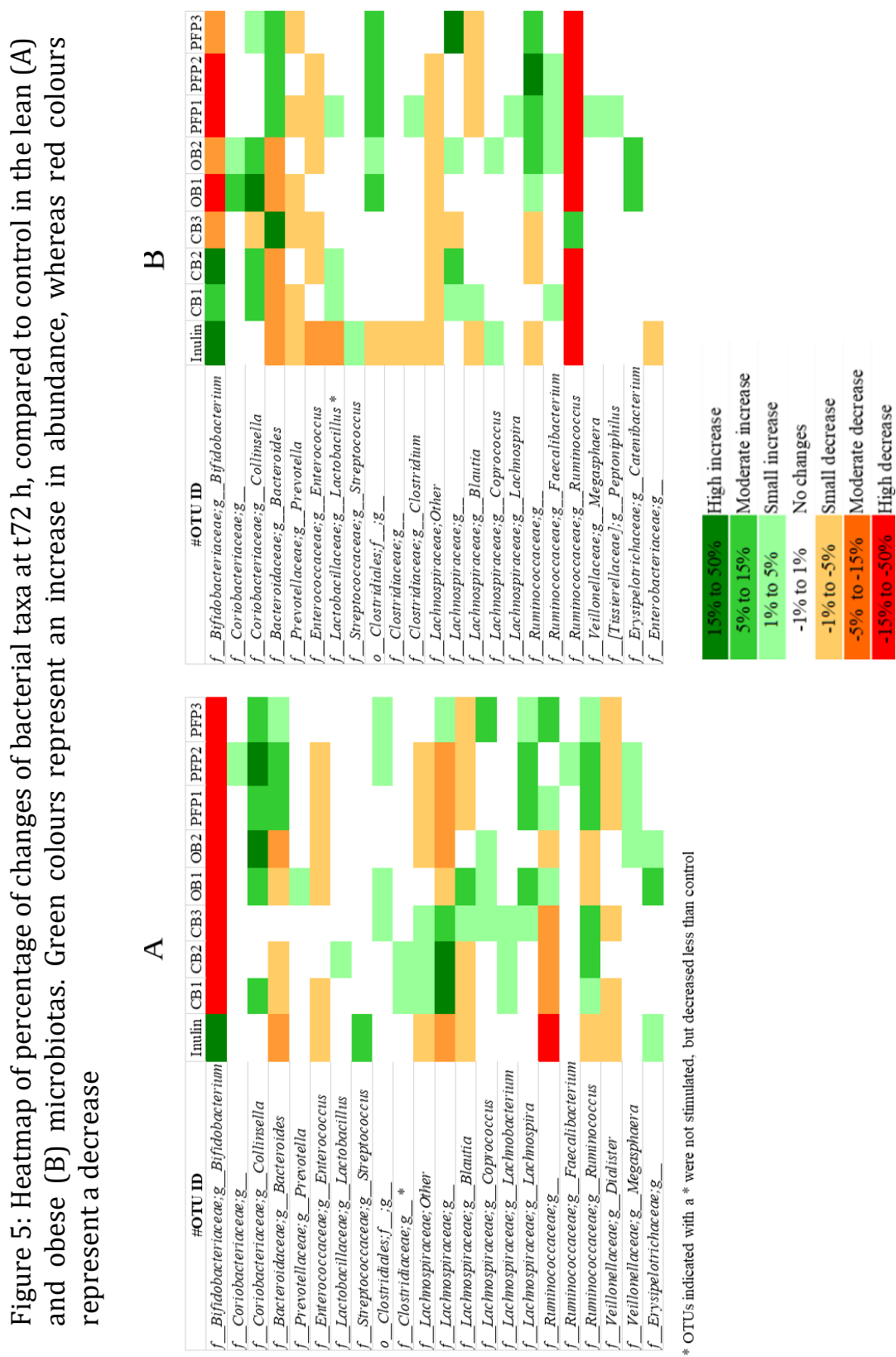
LEfSe analysis showed that Bifidobacterium is a discriminating feature for fructan (Figure 6). In the obese microbiota, CB1 and CB2 were the only substrates (apart from inulin) that were capable of stimulating the growth of this genus (Figure 5B). Even though Bifidobacterium ferments mostly monoand oligosaccharides, some species are capable of degrading complex carbohydrates like starch (51), producing mainly acetate and lactate (52).
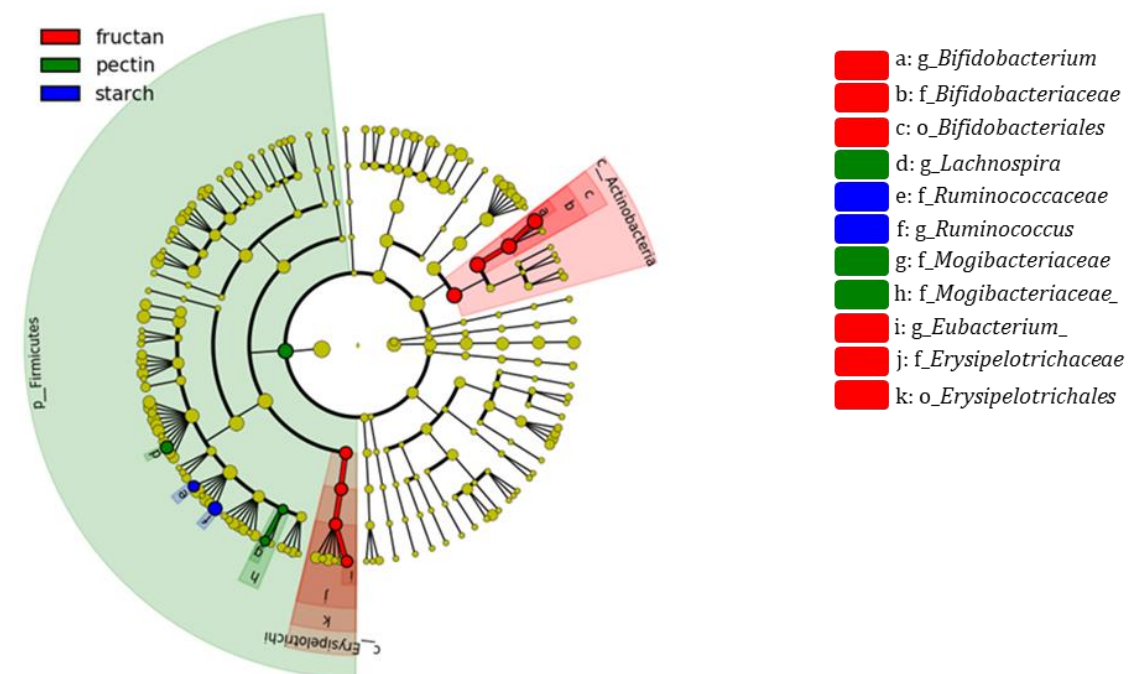

Figure 6: LEfSe with the different test compounds showing which bacterial groups were significantly different among samples.

Our results, therefore, corroborate the literature, because CB1 and CB2 were mainly composed of starch and in the obese microbiota, they induced the production of acetate and lactate.

Spearman correlation analyses indicated a positive association between bifidobacteria and the presence of glucose $(r h o=0.68 ; q$-value $=0.00021)$. Bifidobacterium could also be increased due to cross-feeding with butyrate producing bacteria that release smaller carbohydrates resulting from their utilization of complex polysaccharides (53), which may explain the positive association found between bifidobacteria and butyrate (rho=0.53; qvalue=0.007). Interestingly, in our previous study with cassava bagasse, although fermentation of cassava bagasse by the obese microbiota produced an increase in the abundance of Bifidobacterium, a stronger bifidogenic effect was observed by fermentation of the lean microbiota (54). Despite some differences between the two studies, still cassava bagasses (CB1 and CB2) were shown here to exert a beneficial effect on the obese microbiota regarding the 
stimulation of the genus Bifidobacterium. The genus Bifidobacterium is known by its positive correlation with health status of host and several studies have shown that the genus Bifidobacterium is decreased in several different disease states $(55,56)$. Our results suggest that cassava bagasses (CB1 and CB2) could be used by the obese population with the objective to increase the abundance of bifidobacteria and consequently, improve health status (not tested here). A study with 98 subjects (lean, overweight and obese individuals) revealed a significant lower faecal concentration of the genus Bifidobacterium in the obese individuals, compared to the other two groups (57). Research performed with purified pectic oligosaccharides (POS) isolated from orange peels revealed that, where orange bagasses reduced the abundance of Bifidobacterium or did not present any influence on Lactobacillus, POS had a positive effect on the growth of these two genera (58). Treatment of orange peels which resulted in purified oligosaccharides clearly influenced the final outcome. Although purification of oligosaccharides represents another way to use food by-products with the goal to transform it into a functional food, our objective was to test the raw byproduct in order to decrease food wastage and use it without any extra processing.

\section{Bacteroides}

The genus Bacteroides was stimulated upon fermentation of passion fruit peels, either when the lean or obese microbiota was used. The effects were more prominent for the obese microbiota (Figure 5B), which at the end of the experiment led to higher abundances of Bacteroides than in the lean microbiota (Figure 7). A positive association between Bacteroides and the presence of xylose (rho=0.79; q-value $=7.09^{*} 10^{-06}$ ) and the presence of fucose (rho=0.63; qvalue $=0.003$ ) was found. Xylose and fucose are sugars present in non-starch polysaccharides (NSP) such as hemicellulose and pectin. However, there was no significant association between Bacteroides and pectin. Other studies demonstrated that Bacteroides was enriched with pectin availability $(24,59)$. It is worth to highlight, however, that pectins are complex plant heteropolysaccharides, with major structural differences between various sources. Other characteristics of pectins, like composition of neutral sugars and distribution of homogalacturonan and rhamnogalacturonan fractions might have an effect on the enrichment of diverse bacterial groups. Another in vitro study by us showed that Bacteroides in general was enriched with pectin feeding, but specific species (B. uniformis, B. plebeius and B. ovatus) demonstrated an inconsistent modulation among all different pectins tested (Chapter 7). This might be hypothesized to be due to the highly competitive 
environment, or even specific structural preferences by bacterial groups. This may explain why Bacteroides were only enriched with passion fruit peels and not orange bagasses, although the latter contained higher amounts of pectin (Table 1). It appears that other characteristics of pectins are crucial for it to be utilized by Bacteroides. We also found that Bacteroides was positively associated with degree of acetylation (DA) (rho=0.71; q-value=0.0003), and orange bagasses had low DA (Table 1). (26). Corroborating with the literature, a positive association between Bacteroides and propionate was found (qvalue $=0.03$; rho $=0.51$ ), whereas a negative association with butyrate was detected (q-value $=3.84 * 10^{-05}$; rho $=-0.68$ ).

\section{Faecalibacterium}

The genus Faecalibacterium only increased in abundance upon PFP2 fermentation in the lean microbiota, with a final abundance of $4 \%$. In the obese microbiota CB1, OB2, PFP1 and PFP2 fermentation caused a small increase in Faecalibacterium $(\sim 1.5 \%)$ and the final abundances were $2.9 \%, 3 \%, 3.7 \%$ and $3.3 \%$, respectively (Figure 7B). Its only known species, F. prausnitizii is an antiinflammatory commensal bacterium. Many studies showed inverse correlations between inflammatory bowel disease (IBD) and F. prausnitzii $(60,61)$. Although the exact protective mechanisms are not completely understood, it is believed that the anti-inflammatory property of $F$. prausnitzii is amongst others due to butyrate production, since this species is one of the most abundant butyrate producers in the gut (60). Butyrate has been shown to improve gut permeability through the modulation of expression of tight junction proteins (23). As previously mentioned, obesity is characterized by low grade inflammation that might be caused by the translocation of bacterial LPS due to leaky gut (4).

\section{Catenibacterium}

The growth of the genus Catenibacterium was stimulated only when the obese microbiota was fed with orange bagasses (Figure 5B). This genus produces mainly acetate and lactate (62). A study comparing the effects of dietary habits from Indian and Chinese individuals on microbiota composition suggested that Catenibacterium, amongst others, might have the potential to be used as a microbial biomarker (63). This genus was enriched in faecal samples from Indian individuals, whose vegetarian diet was based on whole grains and plants (63). 
A

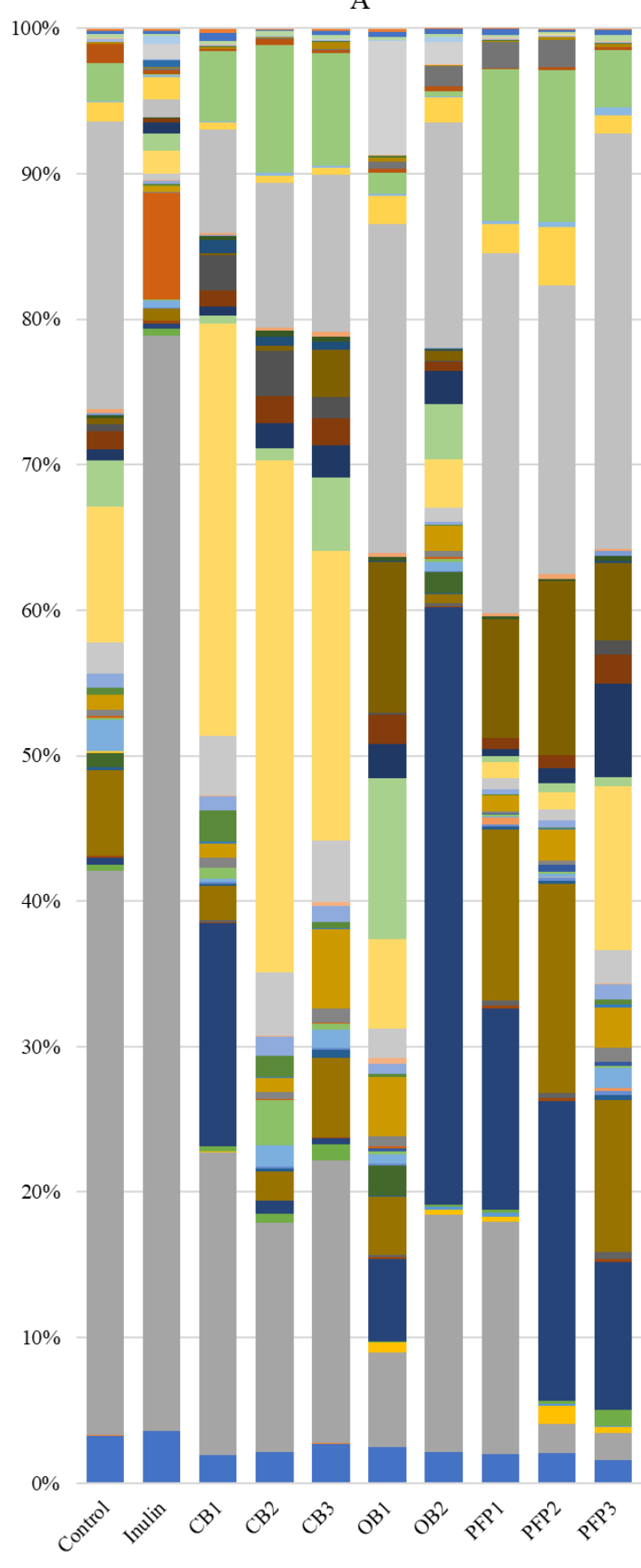

$f \quad$ Enterobacteriaceae; $g$ _Klebsiella

- $f$ Enterobacteriaceae; $g$

$\square$ _Alcaligenaceae; $g$ _Sutterella

$f$ _Erysipelotrichaceae;g_[Eubacterium]

$f$ Erysipelotrichaceae;g Holdemania

$f$ Erysipelotrichaceae; $g$

$f$ [Tissierellaceae];g_Peptoniphilus

$f$ [Tissierellaceae]; $g$ Anaerococcus

$f[$ [Mogibacteriaceae];g

- $f$ Veillonellaceae; $g$ Veillonella

$f$ Veillonellaceae;g_Phascolarctobacterium

- $f$ __ Veillonellaceae;g__Megasphaera

$f$ Veillonellaceae; $g$ Dialister

$f$ Veillonellaceae; $g$

$f$ Ruminococcaceae;g Ruminococcus

$f$ _Ruminococcaceae;g_Oscillospira

$\square$ __Ruminococcaceae; $g \_$Faecalibacterium

$f$ Ruminococcaceae; $g$

$f$ Ruminococcaceae; Other

$f$ Peptostreptococcaceae;g Peptostreptococcus

- $f$ _Lachnospiraceae; $g$ [Ruminococcus]

- $f$ _Lachnospiraceae; $g$ __Roseburia

$f$ Lachnospiraceae; $g$ Lachnospira

$f$ _Lachnospiraceae;g_Lachnobacterium

$f$ Lachnospiraceae; $g$ Dorea

- $f$ L_Lachnospiraceae; $g$ _Coprococcus

$f$ Lachnospiraceae;g Blautia

$f$ Lachnospiraceae; $g$ __ Anaerostipes

$f$ Lachnospiraceae; $g$

$f$ Lachnospiraceae; Other

$f \_$Eubacteriaceae;g_Pseudoramibacter_Eubacterium

$f \_$Clostridiaceae;g_Clostridium

$f$ Clostridiaceae; $g$

- $f$ _Clostridiaceae; Other

- o_Clostridiales; $f ; g$

o Clostridiales; Other; Other

- Streptococcaceae;g Streptococcus

- $f$ Lactobacillaceae;g_Pediococcus

$f$ _Lactobacillaceae;g_L_Lactobacillus

$f$ Enterococcaceae;g_Enterococcus

$f$ Enterococcaceae; $g$

o L Lactobacillales; $f$; $g$

o $\quad$ Bacteroidales; $f \overline{S 24-7} ; g$

$f$ Rikenellaceae; $g$

- $f$ Prevotellaceae; $g$ Prevotella

- $f$ _ Porphyromonadaceae; $g$ _ Parabacteroides

$f$ Bacteroidaceae;g Bacteroides

$f$ Coriobacteriaceae; $g$ Slackia

- f_Coriobacteriaceae;g_Eggerthella

- $f$ Coriobacteriaceae; $g$ _Collinsella

$f$ Coriobacteriaceae; $g$ _ Atopobium

$f$ Coriobacteriaceae; $g$ Adlercreutzia

$f$ Coriobacteriaceae; $g$

$f$ Bifidobacteriaceae; $g$ Bifidobacterium

- $f$ _Actinomycetaceae;g_Actinomyces

- Unassigned 
B

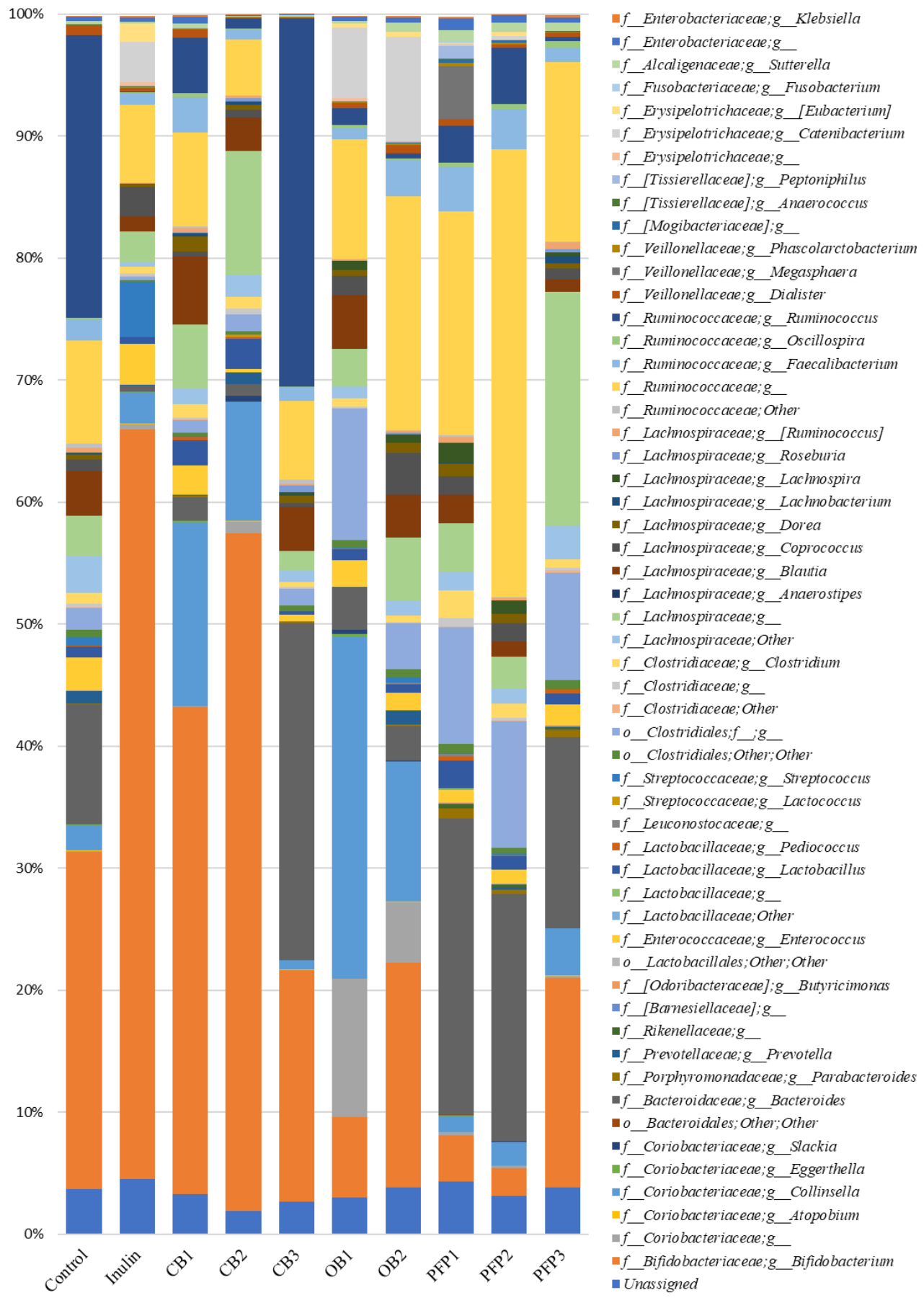

Figure 7: Microbiota composition after fermentation period with the different test compounds (\% of bacterial abundance), in lean (A) and obese (B) microbiotas. 


\section{Collinsella}

In the lean microbiota, Collinsella was stimulated by CB1, orange bagasses and passion fruit peels (Figure 5A). A robust increase in abundance of Collinsella upon fermentation of OB2 was found (increase of 40.5\%) with a final abundance of 41\% (Figure 7A). A similar effect of orange bagasses on Collinsella abundance was observed with the obese microbiota. OB1 enriched the abundance of this genus by $26 \%$, and at the end of the fermentation, Collinsella accounted for $28 \%$ of the total abundance (Figure 7B). A positive correlation between Collinsella and small sugars was detected (rho=0.52; q-value $=0.03$ ), explaining the increase in this genus by orange bagasses, rich in small sugars (Table 1). Passion fruit peels stimulated the increase in abundance of Collinsella in the lean microbiota. A negative association between DA and Collinsella was found (rho=-0.49; q-value=0.04), which might explain the high abundance of Collinsella with addition of PFPs in the lean microbiota. This negative association, nevertheless, could not explain why in the obese microbiota Collinsella was only stimulated by PFP3. Some studies show a correlation between Collinsella and lipid and glucose metabolism. E.g., a study performed with overweight and obese pregnant women found a positive correlation between Collinsella, triglycerides and VLDL cholesterol (64). Another study with non-diabetic, prediabetic and type 2 diabetic patients found an increased abundance of Collinsella in diabetic patients (65). A clinical trial with healthy adults revealed a positive correlation between Collinsella and serum cholesterol (66). The exact mechanism by which the gut microbiota affects host lipid/glucose metabolism is not completely understood. A possible explanation is through bacterial modification of bile acids. Collinsella has the ability to deconjugate bile acids (51). Tran et al. (67) and others (56) proposed a mechanism by which gut microbiota affects bile acid metabolism with consequences for host weight. Under normal conditions, bile acids in human faeces are dominated by secondary bile acids. The researchers proposed that a normal gut microbiota is required for the production of colonic bile acids with higher amounts of secondary bile acids (67). Secondary bile acids are ligands of the cell membrane receptor G-protein-coupled bile acid receptor (TGR5), which also triggers the release of the satiety hormones GLP-1 and PYY. These satiety hormones are also recognized by their effects on gut barrier function (68). In the case of dysbiosis, however, bacterial groups that have the enzyme bile salt hydrolase (BSH) which is necessary for bile acid deconjugation, may be reduced or absent (67). Therefore, the production of secondary bile acids is impaired, and colonic bile acid profiles would be enriched with primary bile acids (67). Thus, activation of TGR5 would not occur optimally, which could lead to a leaky 
gut (67), and in this manner contribute to metabolic endotoxemia. The released proinflammatory mediators will phosphorylate insulin receptor substrate (IRS1) from the insulin receptor. As a result, this leads to the impairment of insulin action and causes insulin resistance (69). The consequent insulin resistance will favour hyperinsulinemia and thus increase hepatic and adipose tissue lipid storage (4). Although the above-mentioned studies correlated Collinsella with negative aspects related to lipid/glucose metabolism, it remains unclear whether the increased abundance is detrimental to the host or if this is a consequence of dysbiosis caused by environmental factors such as bad eating habits, or use of antibiotics, which increase the abundance of Collinsella in order to ameliorate lipid metabolism. Because Collinsella has the ability to deconjugate bile acids, its increase could represent a protective manner to counteract the harmful effects of a high fat diet, for instance.

\section{Ruminococcus}

Fermentation of the three tested cassava bagasses enriched the abundance of Ruminococcus in the lean microbiota, (Figure 5A). In the obese microbiota, however, fermentation of CB1 and CB2 caused a large decrease in Ruminococcus ( $20 \%$ ). CB3, instead, stimulated it by $7 \%$ (Figure $5 \mathrm{~A}$ ), and at $\mathrm{t} 72 \mathrm{~h}$, the microbiota was composed by $30.2 \%$ of the genus Ruminococcus (Figure 7). Some species of Ruminococcus were found to degrade resistant starch (51). We have demonstrated in an in vitro model of the upper gastrointestinal tract (TIM-1) that CB1, CB2 and CB3 were composed on average of $12 \%$ of resistant starch (12). The genus Ruminococcus was inversely associated with small sugars (rho $=-0.49$; q-value $=0.02$ ). Some species of Ruminococcus have the ability to ferment recalcitrant fibres in the colon (70), and therefore might have a preference for such substrates. Fermentation in both microbiotas (lean and obese) caused a decrease in this group when orange bagasses were used as substrate. OB1 contained $22.8 \%$ of small sugars, whilst OB2 had 33.4\% (Table 1), which may explain why Ruminococcus was not stimulated by these substrates. An opposite effect was found in an in vitro study performed with obese microbiota (39). The use of lemon pectin as substrate actually enriched the abundance of Ruminococcaceae family. The substrate used in our study was the whole material remaining after the manufacturing of orange juice (the whole bagasse), not only composed of pectin, but also for instance of small sugars (Table 1), which likely affected the bacterial dynamics, explaining the discrepancy between studies. As for cassava bagasse, fermentation of passion fruit peels caused opposite effects on Ruminococcus abundance in lean and obese microbiotas. Compared to control, lean microbiota 
fermentation resulted in an increase in Ruminococcus abundance when all three passion fruit peels were tested (Figure 5A). The opposite effect occurred when the obese microbiota was used. Passion fruit peel fermentation caused a high decrease (between 15\% and 50\%) in the abundance of this genus. Ruminococcus has been inversely correlated with pro-inflammatory markers, such as interleukin-6 (IL-6) and C-reactive protein (CRP) (71). A human crossover study with 28 healthy participants which consumed three different whole grain test meals for 17 weeks showed a negative correlation between the genus Ruminococcus and CRP (72). Obesity is a metabolic disease characterized by a low-grade inflammation, which contributes to an increased risk to develop insulin resistance and type 2 diabetes (4). A prebiotic which can enrich the abundance of anti-inflammatory species that can contribute to counteracting the negative effects of obesity is highly warranted. It is very intriguing to observe that cassava bagasses and passion fruit peels were only effective in increasing the abundance of Ruminococcus in lean microbiota. Apart from CB3, all other compounds decreased Ruminococcus abundance at $\mathrm{t} 72 \mathrm{~h}$ (Figure 5). It remains unclear what caused such differences.

\section{Lachnospiraceae}

Cassava bagasses fermentation in the lean microbiota caused a unique effect on the genus Lachnobacterium (Figure 7A). They caused a small increase in this genus, and the final abundance was $2.4 \%, 3.1 \%$ and $1.5 \%$ in CB1, CB2 and CB3, respectively. None of the other substrates modulated this family, not in the lean nor in the obese microbiota. Spearman correlation showed a positive association between Lachnobacterium and the presence of glucose in the substrate (rho $=0.78$; q-value $=6.2 * 10^{-06}$ ) and a negative correlation with pectin (rho=-0.55; q-value=0.01). The family Lachnospiraceae was found to be inhibited by citrus pectin in an in vitro study that used microbiota from obese individuals (39). Young mice which received subtherapeutic antibiotic therapy showed substantial changes in microbiome, that resulted in increased adiposity (73). The Lachnospiraceae family appeared in high proportions in the antibiotic-treated group (73). Lachnobacterium bovis, the unique species from the genus Lachnobacterium, isolated from rumen and faeces of cattle, was found to ferment primarily glucose (74). The role of Lachnobacterium in the human gut remains to be elucidated. A study with IBD patients found that the relative abundance of Lachnobacterium was reduced in patients with ulcerative colitis (75). 


\section{Blautia}

It is worth noting that both in the lean and obese microbiota, fermentation of passion fruit peels caused a reduction in the abundance of Blautia (Figure 5). At the end of the fermentation, abundance of Blautia in PFP1, PFP2 and PFP3 was $0.4 \%, 0.6 \%$ and $0.6 \%$ in the lean microbiota, respectively. In the obese microbiota the final abundance was higher than in the lean microbiota $-2.3 \%$, $1.2 \%$ and $1 \%$, respectively (Figure $7 \mathrm{~B}$ ). Blautia uses hydrogen and carbon dioxide to form acetate (51). Studies are showing contradictory results of Blautia on obesity. For instance, a research with obese and non-obese individuals found a positive association between obesity and Blautia hydrogenotorophica, but also a positive association between Blautia wexlerae and the non-obese group (76). Our analysis was performed at the genus level, and therefore it was not possible to distinguish species and relate it with the lean or obese microbiota.

\section{Conclusion}

Our results showed that fermentation of different types of food by-products were metabolized differently by the lean and obese microbiotas. Regarding microbiota composition, a clear separated cluster at the beginning of the experiments between lean and obese microbiota was found, and at the end of the fermentation period, the clustering within lean and obese samples disappeared, and instead presence of primary substrate in the tested substrates (pectin, starch and fructan) drove a new clustering. This new clustering showed the potential of food by-product in driving the obese microbiota into a healthier profile, similar to lean.

\section{Cassava bagasse}

Cassava bagasses were the only substrates (apart from inulin) that enriched the beneficial genus Bifidobacterium. The production of total SCFA by cassava bagasses by the obese microbiota was higher than control and inulin. SCFA are related to the release of satiety hormones, and therefore this by-product might represent a tool to tackle obesity.

\section{Orange bagasse}

Orange bagasses stimulated the growth of the genera Collinsella and Catenibacterium. Collinsella is thought to be involved in lipid metabolism. It remains to be elucidated whether this is beneficial or detrimental to host health. Generation of SCFA was similar to control and inulin, indicating the utilization of this by-products by the gut microbiota. 


\section{Passion fruit peel}

Passion fruit peels were the substrates that yielded smaller amounts of total SCFA and intermediate organic acids (lactate and succinate), and produced more BCFA, which together does not represent the best scenario in bacterial fermentation and host health. However, passion fruit peels fermentation resulted in a microbiota with the highest diversity and evenness, a positive trait regarding host health. Fermentation of passion fruit peels, which had higher protein content, stimulated the enrichment of Bacteroides, which may explain higher amounts of BCFA production. Additionally, Ruminococcus were increased only in the lean microbiota fed with passion fruit peels. Ruminococcus species are known to use recalcitrant fibres for energy extraction, and a possible explanation is that the obese microbiota was not used to this type of substrate and therefore did not have: i) the necessary species to efficiently degrade complex fibres and/or ii) the machinery to break down the substrate and make complete use of it.

The results found in this study show that food by-products have the potential to be used as a tool to manipulate not only the microbiota from obese individuals, but also from e.g. healthy individuals. The use of food by-products may represent a way to give a better destination to these by-products, such as to incorporate them back into food products as functional ingredients. The results of the present mechanistic study show promise that these by-products could be an important step forward to tackle obesity and at the same time decrease the waste of valuable food material and consequently environmental pollution. Moreover, they are inexpensive and a non-invasive way to be used as a dietary intervention to improve health. Further experiments, either in vitro or in vivo are suggested to confirm our findings and contribute to elucidate the interactions between gut microbiota, obesity and utilization of complex polysaccharides. For instance, combinations of the food by-products could be tested to study their synergistic or antagonistic effect.

\section{Conflict of interest}

The authors declare that there are no conflicts of interest.

\section{Financial support}

Carlota Bussolo de Souza received a PhD grant from CNPq (National Council for Scientific and Technological Development - Brazil) under the program "Science without Borders" (grant number 246027/2012-6). The study was partly funded by the Centre for Healthy Eating \& Food Innovation (HEFI) of 
Maastricht University - campus Venlo. This research has been made possible with the support of the Dutch Province of Limburg.

\section{Acknowledgments}

We would like to acknowledge Freddy Troost and Bouke Salden from Maastricht University for contacting and collecting faecal donation from obese volunteers at Maastricht University Medical Center. Furthermore, we would like to thank the companies that kindly sent samples for this research Alimentos Grazimara, Companhia Lorenz, Chá\&Cia, Phytomare, and Orafti. We thank Prof. Dr. Thiago Cardoso de Souza from Rio Grande do Norte Federal University - Brazil - for his assistance with R. We kindly acknowledge Geraldo Bussolo and Zélia Maria Bussolo for collecting samples of orange bagasse and passion fruit peel (OB2 and PFP3) from Brazil. The participation of volunteers that donated their faeces and made this study possible is greatly appreciated. 


\section{References}

1. World Health Organization. Obesity and overweight. World Health Organization; 2016.

2. Turnbaugh PJ, Ley RE, Mahowald M a, Magrini V, Mardis ER, Gordon JI. An obesity-associated gut microbiome with increased capacity for energy harvest. Nature. 2006;444(7122):1027-31.

3. Ley RE, Turnbaugh PJ, Klein S, Gordon JI. Microbial ecology: human gut microbes associated with obesity. Nature. England; 2006 Dec;444(7122):1022-3.

4. Cani PD, Amar J, Iglesias MA, Poggi M, Knauf C, Bastelica D, et al. Metabolic endotoxemia initiates obesity and insulin resistance. Diabetes. United States; 2007 Jul;56(7):1761-72.

5. Dao MC, Everard A, Aron-Wisnewsky J, Sokolovska N, Prifti E, Verger EO, et al. Akkermansia muciniphila and improved metabolic health during a dietary intervention in obesity: relationship with gut microbiome richness and ecology. Gut. 2016;65(3):426-36.

6. da Silva ST, dos Santos CA, Bressan J. Intestinal microbiota; relevance to obesity and modulation by prebiotics and probiotics. Nutr Hosp. Spain; 2013;28(4):1039-48.

7. Martinez RC, Bedani R, Saad SM. Scientific evidence for health effects attributed to the consumption of probiotics and prebiotics: an update for current perspectives and future challenges. $\mathrm{Br} \mathrm{J}$ Nutr. 2015;114(12):1993-2015.

8. Pandey A, Soccol CR, Nigam P, Soccol VT, Vandenberghe LPS, Mohan R. Biotechnological potential of agro-industrial residues. II: cassava bagasse. Bioresour Technol. 2000;74(1):81-7.

9. Martínez R, Torres P, Meneses MA, Figueroa JG, Pérez-Álvarez JA, ViudaMartos M. Chemical, technological and in vitro antioxidant properties of mango, guava, pineapple and passion fruit dietary fibre concentrate. Food Chem. 2012;135(3):1520-6.

10. Juul F, Hemmingsson E. Trends in consumption of ultra-processed foods and obesity in Sweden between 1960 and 2010. Public Health Nutr. England; 2015 Dec;18(17):3096-107.

11. Louzada ML da C, Baraldi LG, Steele EM, Martins APB, Canella DS, Moubarac J-C, et al. Consumption of ultra-processed foods and obesity in Brazilian adolescents and adults. Prev Med (Baltim). United States; 2015 Dec;81:9-15.

12. de Souza CB, Jonathan M, Saad SMI, Schols HA, Venema K. Characterization and in vitro digestibility of by-products from Brazilian food industry: cassava bagasse, orange bagasse and passion fruit peel. Bioact Carbohydrates Diet Fibre. 2018 Oct 1; 16:90-9.

13. Minekus M, Smeets-Peeters M, Bernalier A, Marol-Bonnin S, Havenaar R, Marteau P, et al. A computer-controlled system to simulate conditions of the large intestine with peristaltic mixing, water absorption and absorption of fermentation products. Appl Microbiol Biotechnol. 
1999;53(1):108-14.

14. Rehman A, Heinsen F-A, Koenen ME, Venema K, Knecht H, Hellmig S, et al. Effects of probiotics and antibiotics on the intestinal homeostasis in a computer controlled model of the large intestine. BMC Microbiol. BioMed Central Ltd; 2012;12(1):47.

15. Kovatcheva-Datchary P, Egert M, Maathuis A, Rajilić-Stojanović M, De Graaf AA, Smidt H, et al. Linking phylogenetic identities of bacteria to starch fermentation in an in vitro model of the large intestine by RNAbased stable isotope probing. Environ Microbiol. 2009;11(4):914-26.

16. Aguirre M, De Souza CB, Venema K. The gut microbiota from lean and obese subjects contribute differently to the fermentation of arabinogalactan and inulin. PLoS One. 2016 Jul 1;11(7).

17. Aguirre M, Eck A, Koenen ME, Savelkoul PHM, Budding AE, Venema K. Evaluation of an optimal preparation of human standardized fecal inocula for in vitro fermentation studies. J Microbiol Methods. Netherlands; 2015 Oct;117:78-84.

18. Aguirre M, Ramiro-Garcia J, Koenen ME, Venema K. To pool or not to pool? Impact of the use of individual and pooled fecal samples for in vitro fermentation studies. J Microbiol Methods. 2014;107C:1-7.

19. Venema K, Nuenen M van, Smeets-Peeters M, Minekus M, Havenaar R. TNO's in vitro large intestinal model: an excellent screening tool for functional food and pharmaceutical research. Ernährung Nutr. 2000;24(12):559-64.

20. Caporaso JG, Kuczynski J, Stombaugh J, Bittinger K, Bushman FD, Costello EK, et al. QIIME allows analysis of high-throughput community sequencing data. Vol. 7, Nature methods. United States; 2010. p. 335-6.

21. $\mathrm{R}$ Core Team. A language and environment for statistical computing. $\mathrm{R}$ Foundation for Statistical Computing. Viena, Austria; 2013.

22. Segata N, Izard J, Waldron L, Gevers D, Miropolsky L, Garrett WS, et al. Metagenomic biomarker discovery and explanation. Genome Biol. England; 2011 Jun;12(6):R60.

23. Canfora EE, Jocken JW, Blaak EE. Short-chain fatty acids in control of body weight and insulin sensitivity. Nat Rev Endocrinol. Nature Publishing Group; 2015;11(10):577-91.

24. Aguirre M, Jonkers DMAE, Troost FJ, Roeselers G, Venema K. In vitro characterization of the impact of different substrates on metabolite production, energy extraction and composition of gut microbiota from lean and obese subjects. PLoS One. United States; 2014;9(11):e113864.

25. Turnbaugh PJ, Ley RE, Mahowald MA, Magrini V, Mardis ER, Gordon JI. An obesity-associated gut microbiome with increased capacity for energy harvest. Nature. 2006;444(7122):1027-31.

26. Chambers ES, Morrison DJ, Frost G. Control of appetite and energy intake by SCFA: what are the potential underlying mechanisms? Proc Nutr Soc. England; 2015 Aug;74(3):328-36.

27. Kondo T, Kishi M, Fushimi T, Ugajin S, Kaga T. Vinegar intake reduces 
body weight, body fat mass, and serum triglyceride levels in obese Japanese subjects. Biosci Biotechnol Biochem. England; 2009 Aug;73(8):1837-43.

28. Beh BK, Mohamad NE, Yeap SK, Ky H, Boo SY, Chua JYH, et al. Antiobesity and anti-inflammatory effects of synthetic acetic acid vinegar and Nipa vinegar on high-fat-diet-induced obese mice. Sci Rep. London: Nature Publishing Group UK; 2017 Jul 27;7:6664.

29. van der Beek CM, Canfora EE, Lenaerts K, Troost FJ, Damink SWMO, Holst JJ, et al. Distal, not proximal, colonic acetate infusions promote fat oxidation and improve metabolic markers in overweight/obese men. Clin Sci (Lond). England; 2016 Nov;130(22):2073-82.

30. Perry RJ, Peng L, Barry NA, Cline GW, Zhang D, Cardone RL, et al. Acetate mediates a microbiome-brain- $\beta$ cell axis promoting metabolic syndrome. Nature. 2016 Jun 9;534(7606):213-7.

31. Al-Lahham S, Peppelenbosch MP, Roelofsen H, Vonk RJ, Venema K. Biological effects of propionic acid in humans; metabolism, potential applications and underlying mechanisms. Biochim Biophys Acta. 2010;1801(11):1175-83.

32. Macfarlane S, Macfarlane GT. Regulation of short-chain fatty acid production. Proc Nutr Soc. 2003;62:67-72.

33. De Vadder F, Kovatcheva-Datchary P, Goncalves D, Vinera J, Zitoun C, Duchampt A, et al. Microbiota-Generated Metabolites Promote Metabolic Benefits via Gut-Brain Neural Circuits. Cell. 2014 Jan 16;156(1-2):84-96.

34. Nohr MK, Pedersen MH, Gille A, Egerod KL, Engelstoft MS, Husted AS, et al. GPR41/FFAR3 and GPR43/FFAR2 as cosensors for short-chain fatty acids in enteroendocrine cells vs FFAR3 in enteric neurons and FFAR2 in enteric leukocytes. Endocrinology. United States; 2013 Oct;154(10):3552-64.

35. Samuel BS, Shaito A, Motoike T, Rey FE, Backhed F, Manchester JK, et al. Effects of the gut microbiota on host adiposity are modulated by the short-chain fatty-acid binding G protein-coupled receptor, Gpr41. Proc Natl Acad Sci U S A. United States; 2008 Oct;105(43):16767-72.

36. Tolhurst G, Heffron H, Lam YS, Parker HE, Habib AM, Diakogiannaki E, et al. Short-chain fatty acids stimulate glucagon-like peptide-1 secretion via the G-protein-coupled receptor FFAR2. Diabetes. United States; 2012 Feb;61(2):364-71.

37. Bianchi F, Lopes NP, Adorno MAT, Sakamoto IK, Genovese MI, Saad SMI, et al. Impact of combining acerola by-product with a probiotic strain on a gut microbiome model. Int J Food Sci Nutr. England; 2018 Aug;1-13.

38. Macagnan FT, dos Santos LR, Roberto BS, de Moura FA, Bizzani M, da Silva LP. Biological properties of apple pomace, orange bagasse and passion fruit peel as alternative sources of dietary fibre. Bioact Carbohydrates Diet Fibre. Elsevier; 2015;6(1):1-6.

39. Bianchi F, Larsen N, de Mello Tieghi T, Adorno MAT, Kot W, Saad SMI, et al. Modulation of gut microbiota from obese individuals by in vitro 
fermentation of citrus pectin in combination with Bifidobacterium longum BB-46. Appl Microbiol Biotechnol. Germany; 2018 Aug.

40. Canani RB, Costanzo M Di, Leone L, Pedata M, Meli R, Calignano A. Potential beneficial effects of butyrate in intestinal and extraintestinal diseases. Vol. 17, World journal of gastroenterology. United States; 2011. p. 1519-28.

41. Byrne CS, Chambers ES, Morrison DJ, Frost G. The role of short chain fatty acids in appetite regulation and energy homeostasis. Int J Obes (Lond). England; 2015 Sep;39(9):1331-8.

42. Hatayama H, Iwashita J, Kuwajima A, Abe T. The short chain fatty acid, butyrate, stimulates MUC2 mucin production in the human colon cancer cell line, LS174T. Biochem Biophys Res Commun. 2007;356(3):599-603.

43. Willemsen LEM, Koetsier MA, van Deventer SJH, van Tol EAF. Short chain fatty acids stimulate epithelial mucin 2 expression through differential effects on prostaglandin $\mathrm{E}(1)$ and $\mathrm{E}(2)$ production by intestinal myofibroblasts. Gut. England; 2003 Oct;52(10):1442-7.

44. Hamer HM, Jonkers DMAE, Renes IB, Vanhoutvin SALW, Kodde A, Troost FJ, et al. Butyrate enemas do not affect human colonic MUC2 and TFF3 expression. Eur J Gastroenterol Hepatol. England; 2010 Sep;22(9):113440.

45. Peng L, Li Z-R, Green RS, Holzman IR, Lin J. Butyrate Enhances the Intestinal Barrier by Facilitating Tight Junction Assembly via Activation of AMP-Activated Protein Kinase in Caco-2 Cell Monolayers. J Nutr. American Society for Nutrition; 2009 Sep 14;139(9):1619-25.

46. Yan H, Ajuwon KM. Butyrate modifies intestinal barrier function in IPECJ2 cells through a selective upregulation of tight junction proteins and activation of the Akt signaling pathway. PLoS One. United States; 2017;12(6):e0179586.

47. Flint HJ, Duncan SH, Louis P. 2 - Gut microbial ecology BT - Designing Functional Foods. In: Woodhead Publishing Series in Food Science, Technology and Nutrition. Woodhead Publishing; 2009. p. 38-67.

48. Koenen ME, Cruz Rubio JM, Mueller M, Venema K. The effect of agave fructan products on the activity and composition of the microbiota determined in a dynamic in vitro model of the human proximal large intestine. J Funct Foods. 2016;22:201-10.

49. Lima GC, Vuolo MM, Batista ÂG, Dragano NR V, Solon C, Junior MRM. Passiflora edulis peel intake improves insulin sensitivity, increasing incretins and hypothalamic satietogenic neuropeptide in rats on a highfat diet. Nutrition. 2016;32(7):863-70.

50. Serena C, Ceperuelo-Mallafre V, Keiran N, Queipo-Ortuno MI, Bernal R, Gomez-Huelgas R, et al. Elevated circulating levels of succinate in human obesity are linked to specific gut microbiota. ISME J. England; 2018 Jun;12(7):1642-57.

51. Rajilić-Stojanović M, de Vos WM. The first 1000 cultured species of the human gastrointestinal microbiota. FEMS Microbiol Rev. 
2014;38(5):996-1047.

52. De Graaf A, Venema K. Gaining Insight into Microbial Physiology in the Large Intestine: A Special Role for Stable Isotopes. 2007;53:73-314.

53. Flint HJ, Scott KP, Duncan SH, Louis P, Forano E. Microbial degradation of complex carbohydrates in the gut. Vol. 3, Gut Microbes. 2012. p. 289306.

54. Bussolo de Souza C, Roeselers G, Troost F, Jonkers D, Koenen ME, Venema K. Prebiotic effects of cassava bagasse in TNO's in vitro model of the colon in lean versus obese microbiota. J Funct Foods. Elsevier Ltd; 2014;11(C):210-20.

55. Ignacio A, Fernandes MR, Rodrigues VAA, Groppo FC, Cardoso AL, AvilaCampos MJ, et al. Correlation between body mass index and faecal microbiota from children. Clin Microbiol Infect. England; 2016 Mar;22(3):258.e1-8.

56. Duboc H, Rainteau D, Rajca S, Humbert L, Farabos D, Maubert M, et al. Increase in fecal primary bile acids and dysbiosis in patients with diarrhea-predominant irritable bowel syndrome. Neurogastroenterol Motil. England; 2012 Jun;24(6):513-20, e246-7.

57. Schwiertz A, Taras D, Schafer K, Beijer S, Bos NA, Donus C, et al. Microbiota and SCFA in lean and overweight healthy subjects. Obes (Silver Spring). Nature Publishing Group; 2010;18(1):190-5.

58. Gomez B, Gullon B, Remoroza C, Schols HA, Parajo JC, Alonso JL. Purification, characterization, and prebiotic properties of pectic oligosaccharides from orange peel wastes. J Agric Food Chem. 2014;62(40):9769-82.

59. Tian L, Scholte J, Borewicz K, van den Bogert B, Smidt H, Scheurink AJW, et al. Effects of pectin supplementation on the fermentation patterns of different structural carbohydrates in rats. Mol Nutr Food Res. Germany; 2016 Oct;60(10):2256-66.

60. Miquel S, Martín R, Rossi O, Bermúdez-Humarán LG, Chatel JM, Sokol H, et al. Faecalibacterium prausnitzii and human intestinal health. Curr Opin Microbiol. 2013;16(3):255-61.

61. Machiels K, Joossens M, Sabino J, De Preter V, Arijs I, Eeckhaut V, et al. A decrease of the butyrate-producing species Roseburia hominis and Faecalibacterium prausnitzii defines dysbiosis in patients with ulcerative colitis. Gut. England; 2014 Aug;63(8):1275-83.

62. Kageyama A, Benno Y. Catenibacterium mitsuokai gen. nov., sp. nov., a gram-positive anaerobic bacterium isolated from human faeces. Int J Syst Evol Microbiol. England; 2000 Jul;50 Pt 4:1595-9.

63. Jain A, Li XH, Chen WN. Similarities and differences in gut microbiome composition correlate with dietary patterns of Indian and Chinese adults. AMB Express. Germany; 2018 Jun;8(1):104.

64. Gomez-Arango LF, Barrett HL, McIntyre HD, Callaway LK, Morrison M, Dekker Nitert M. Connections between the gut microbiome and metabolic hormones in early pregnancy in overweight and obese 
women. Diabetes. United States; 2016 Aug;65(8):2214-23.

65. Lambeth SM, Carson T, Lowe J, Ramaraj T, Leff JW, Luo L, et al. Composition, diversity and abundance of gut microbiome in prediabetes and type 2 diabetes. J diabetes Obes. United States; 2015 Dec;2(3):1-7.

66. Lahti L, Salonen A, Kekkonen RA, Salojarvi J, Jalanka-Tuovinen J, Palva A, et al. Associations between the human intestinal microbiota, Lactobacillus rhamnosus GG and serum lipids indicated by integrated analysis of high-throughput profiling data. PeerJ. United States; 2013;1:e32.

67. Tran CD, Grice DM, Wade B, Kerr CA, Bauer DC, Li D, et al. Gut permeability, its interaction with gut microflora and effects on metabolic health are mediated by the lymphatics system, liver and bile acid. Futur Microbiol. 2015;10:1339-53.

68. Cani PD, Everard A, Duparc T. Gut microbiota, enteroendocrine functions and metabolism. Curr Opin Pharmacol. Elsevier Ltd; 2013;13(6):935-40.

69. Cani PD, Possemiers S, Van de Wiele T, Guiot Y, Everard A, Rottier O, et al. Changes in gut microbiota control inflammation in obese mice through a mechanism involving GLP-2-driven improvement of gut permeability. Gut. 2009;58(8):1091-103.

70. Flint HJ, Duncan SH, Louis P. The impact of nutrition on intestinal bacterial communities. Curr Opin Microbiol. England; 2017 Aug;38:5965.

71. van den Munckhof ICL, Kurilshikov A, Ter Horst R, Riksen NP, Joosten LAB, Zhernakova $\mathrm{A}$, et al. Role of gut microbiota in chronic low-grade inflammation as potential driver for atherosclerotic cardiovascular disease: a systematic review of human studies. Obes Rev. England; 2018 Aug.

72. Martinez I, Lattimer JM, Hubach KL, Case JA, Yang J, Weber CG, et al. Gut microbiome composition is linked to whole grain-induced immunological improvements. ISME J. England; 2013 Feb;7(2):269-80.

73. Cho I, Yamanishi S, Cox L, Methe BA, Zavadil J, Li K, et al. Antibiotics in early life alter the murine colonic microbiome and adiposity. Nature. England; 2012 Aug;488(7413):621-6.

74. Whitford MF, Yanke LJ, Forster RJ, Teather RM. Lachnobacterium bovis gen. nov., sp. nov., a novel bacterium isolated from the rumen and faeces of cattle. Int J Syst Evol Microbiol. England; 2001 Nov;51(Pt 6):1977-81.

75. Yilmaz B, Spalinger MR, Biedermann L, Franc Y, Fournier N, Rossel J-B, et al. The presence of genetic risk variants within PTPN2 and PTPN22 is associated with intestinal microbiota alterations in Swiss IBD cohort patients. PLoS One. United States; 2018;13(7):e0199664.

76. Kasai C, Sugimoto K, Moritani I, Tanaka J, Oya Y, Inoue H, et al. Comparison of the gut microbiota composition between obese and nonobese individuals in a Japanese population, as analyzed by terminal restriction fragment length polymorphism and next-generation sequencing. BMC Gastroenterol. BioMed Central; 2015;15(1):100. 


\section{Supplemental material}
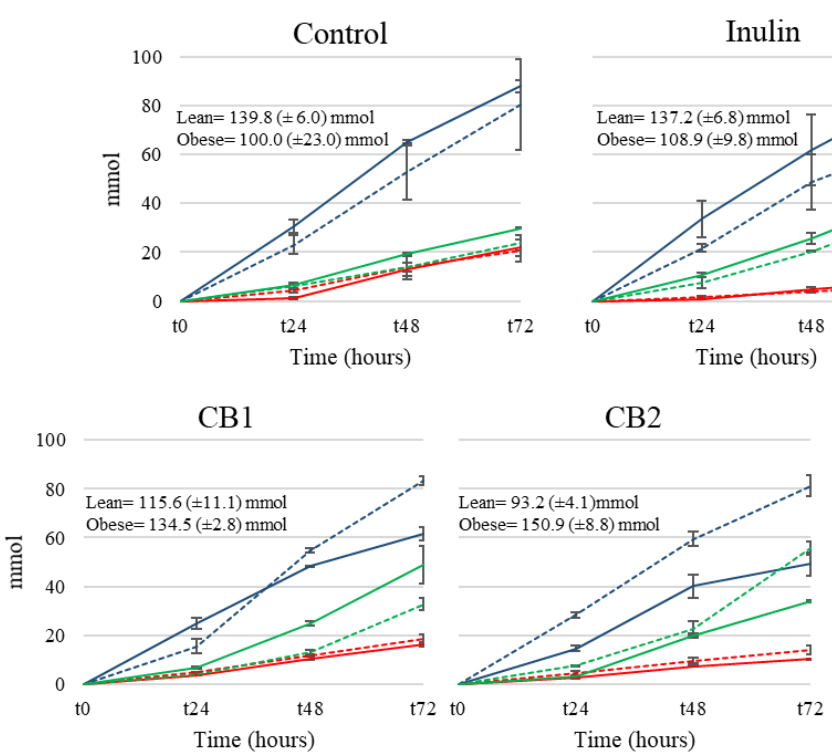

Inulin

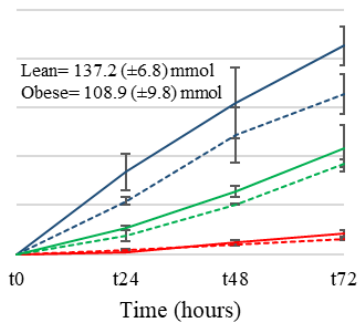

Acetate Lean

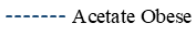

- Propi onate Lean

------- Propi onate Obese

- n-Butyrate Lean

------ n-Butyrate Obese

CB3

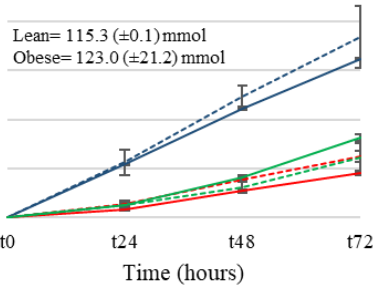

OB2
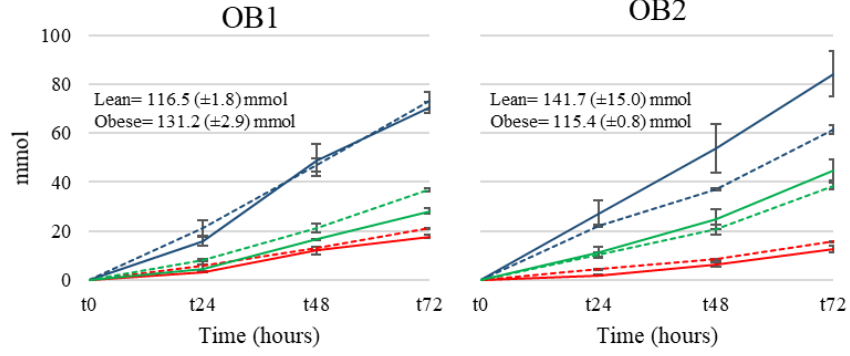

PFP1
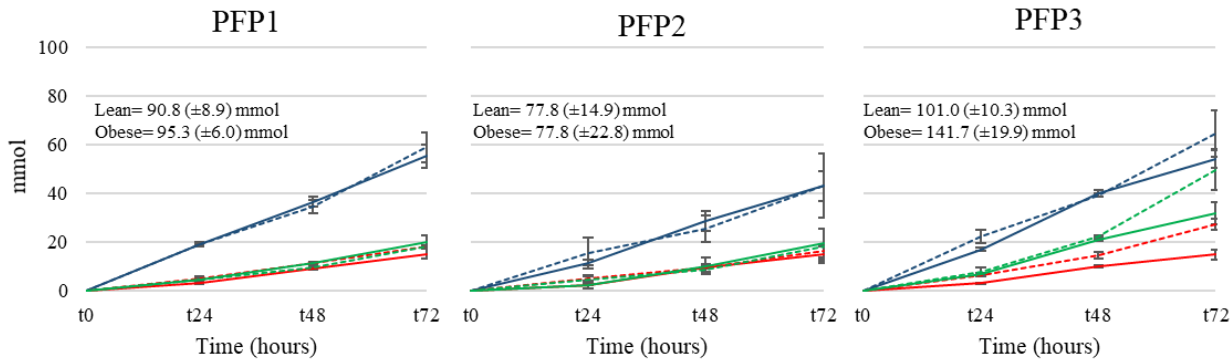

Supplemental Figure 1: Kinetics and total cumulative production of SCFA by the lean and obese microbiota during 72 hours fermentation in TIM-2 with different test compounds. 


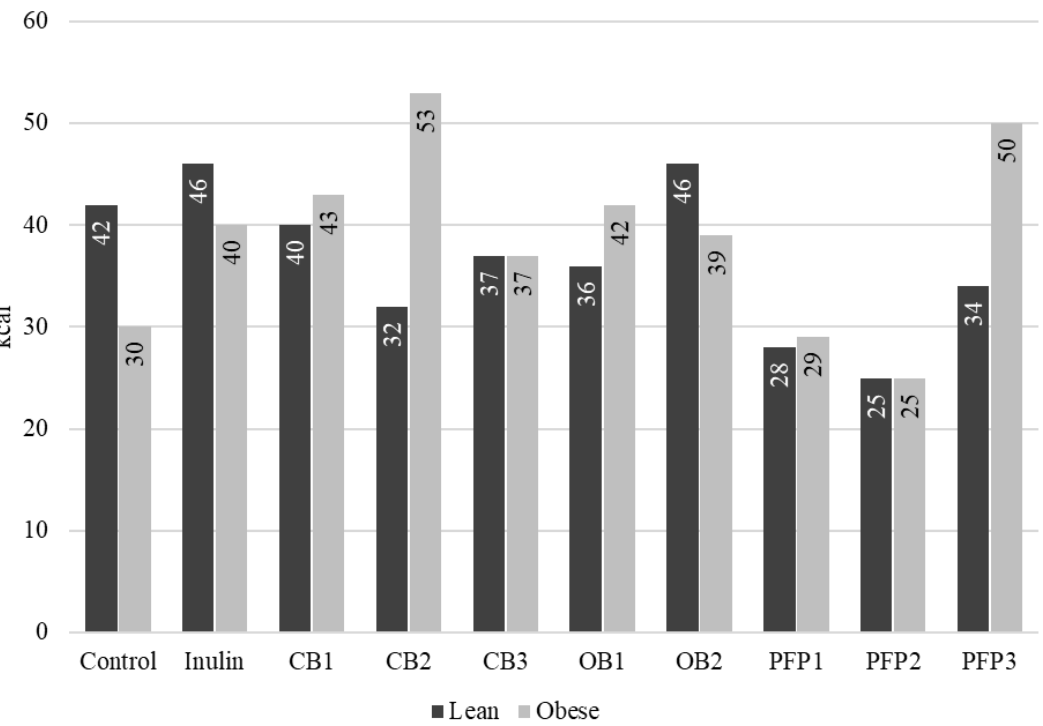

Supplemental Figure 2: Total energy (kcal) production by the lean and obese microbiota after fermentation period with the different test compounds.

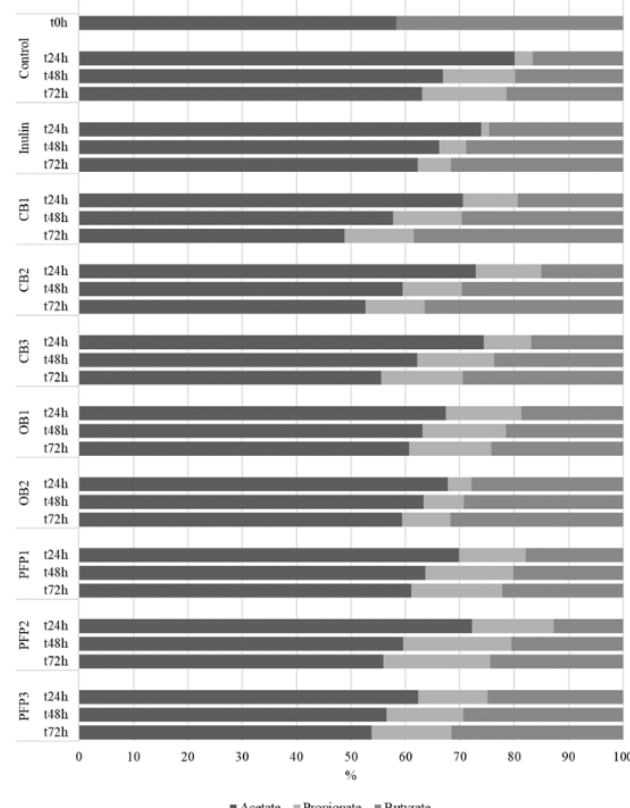

B

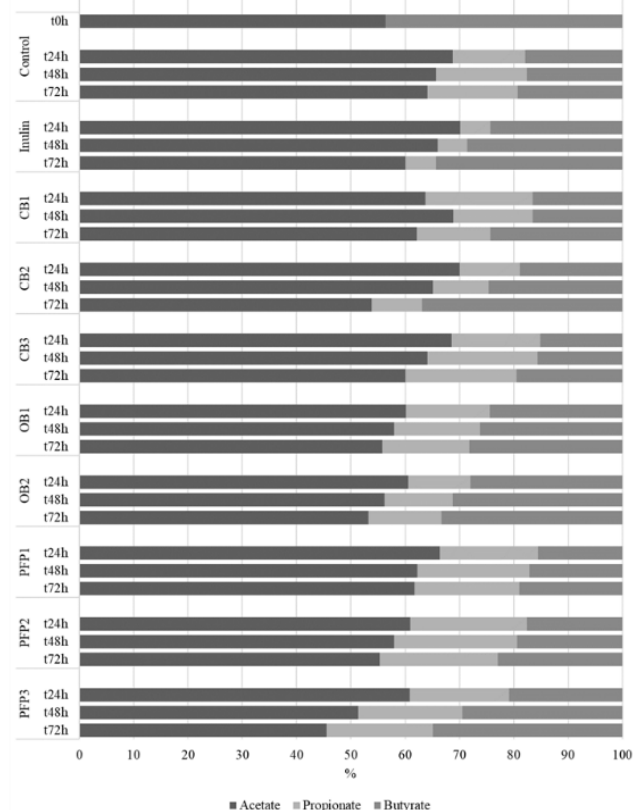

Supplemental Figure 3: Ratio of each SCFA over the experimental period with the different test compounds in the lean (A) and obese (B) microbiotas. 


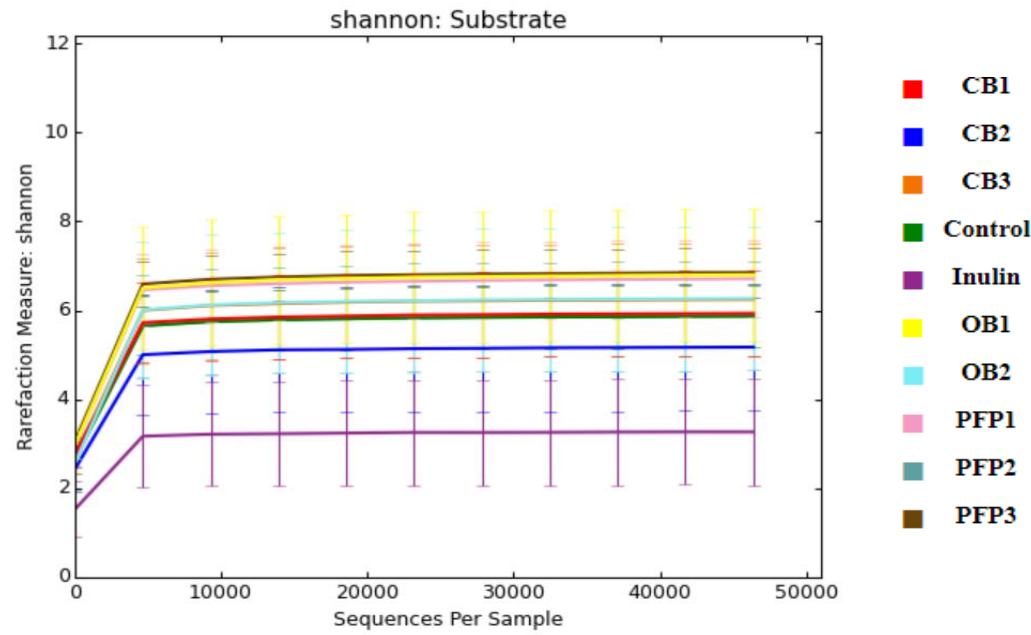

Supplemental Figure 4: Shannon index at $\mathrm{t72}$ h (lean and obese combined) with test compounds. PFP3 had the highest diversity in bacterial population, whereas inulin showed the least diverse composition. 


Chapter 5
Degradation of fibres from fruit by-
products allows selective modulation of
the gut microbiota in an in vitro model of
the proximal colon

Submitted:

Bussolo de Souza C., Jonathan M., Saad S.M.I., Schols H.A., Venema K. Degradation of fibres from fruit by-products allows selective modulation of the gut microbiota in an in vitro model of the proximal colon. 


\section{Abstract}

The potential prebiotic effect of fibres from fruit by-products and their degradation by a healthy adult human gut microbiota was tested in an in vitro colon system (TIM-2). Alcohol insoluble solids of two batches of orange bagasses and passion fruit peels were fed to TIM-2. Standard medium and inulin were used as controls. Orange bagasses had similar chemical composition but differed regarding fermentation profile. A-OB2 resulted in a more diverse bacterial community than A-OB1 and resulted in the production of more SCFA (118.3 mmol and $81.8 \mathrm{mmol}$, respectively), with increased Ruminococcus and Lachnospira. Carbohydrate utilization was higher on A-OB2 probably due to higher ratio soluble to insoluble fibres. Passion fruit peels presented similar chemical composition and fermentation profiling. Bacteroides and Ruminococcus were the main genera stimulated, and their final abundance on A-PFP1 were $6.9 \%$ and $8.5 \%$, respectively, and on A-PFP2 were $9.6 \%$ and $16 \%$, respectively. Negligible lactate and succinate production $(\leq 0.05 \mathrm{mmol})$ may represent slow fermentation, a protective feature against colon cancer. This study provided evidence that fruit by-products have the potential to be used for selective modulation of the gut microbiota. 


\section{Introduction}

Food industries around the globe are responsible for about $38 \%$ of the total food waste produced, which includes food by-products (1). The term "food byproduct" generally refers to edible parts of food that are either discarded during the processing (such as peels, seeds and bagasses), lost, degraded or consumed by pests $(1,2)$. Fruit and vegetables by-products are a source of a variety of dietary fibres, which may be suitable for human consumption (3). Dietary fibres are defined as carbohydrate polymers with a degree of polymerization (DP) of three or more, which are neither digested nor absorbed in the human upper intestine (4). One of the main positive health effects of dietary fibres are related to colonic function, blood glucose- and blood cholesterol reduction (4). Another fundamental function of dietary fibres is to serve as the main energy source to the gut microbiota. In contrast to the host, members of the gut microbiota possess the enzymes necessary to hydrolyse the linkages present in dietary fibres (5). Feeding our gut microbiota with dietary fibres is important because the fermentation of such substrates can (i) stimulate and/or maintain the population of beneficial microbes (e.g. Bifidobacterium), and (ii) stimulate the production of bacterial metabolites (short chain fatty acids - SCFA) (6). Both features are beneficial to the host and essential for the homeostasis of host metabolism (6). Accordingly, dietary fibre has the potential to be used as a tool to manipulate the gut microbiota in order to (re)stablish the equilibrium necessary to maintain host health (7). However, the interplay between gut microbiota fermentation of different substrates and host health is not completely understood. Specifically, the structural complexity of dietary fibre and their influence on the dynamic changes of gut microbe populations remains mostly unknown or underappreciated (7).

We have characterized the isolated fibre from different sources of fruit byproducts (orange bagasses and passion fruit peels) regarding monosaccharide composition, degree of methyl- and acetyl-esterification, protein and pectin content (8). Here, our main goal was to assess the potential prebiotic effect of these fibres in a dynamic in vitro system mimicking the proximal colon (TIM-2), through the analyses of bacterial composition and metabolism. Furthermore, we monitored the fate of these fibres during fermentation (rate and extent of polysaccharides break down by the gut microbiota) through the analyses of intermediate products in order to investigate the bacterial utilization of the different monosaccharides present in these polysaccharides. 


\section{Materials and methods}

\section{Test compounds}

In total, 4 test compounds (plus 2 controls) were used for the fermentation in TIM-2. The test compounds were alcohol insoluble solids (AIS) isolated from two samples of orange bagasses and two samples of passion fruit peels. The orange bagasses were obtained from Chá\&Cia (Jacareí, Brazil) - denoted as AOB1, and from a bulk sale store (Florianópolis, Brazil) - denoted as A-OB2. The passion fruit peels were from Chá\&Cia (Jacareí) - denoted as A-PFP1, and Phytomare (Governador Celso Ramos, Brazil) - denoted as A-PFP2.

More detailed information about the extraction and characterization of the compounds is presented elsewhere (8), and is summarized in Table 1.

Table 1: Constituent monosaccharide composition (weight/weight [w/w] \%), total carbohydrates $(\mathrm{w} / \mathrm{w} \%)$, pectin (\%), protein (\%), degree of methylation (DM) and degree of acetylation (DA) of the alcohol insoluble solids (AIS) from orange bagasses (OBs) and passion fruit peels (PFPs) (8).

\begin{tabular}{|c|c|c|c|c|c|c|c|c|c|c|c|c|c|}
\hline \multirow{2}{*}{$\begin{array}{c}\text { By-product } \\
\text { (AIS) }\end{array}$} & \multicolumn{11}{|c|}{$\%(w / w)$} & \multirow[b]{2}{*}{ DM } & \multirow[b]{2}{*}{ DA } \\
\hline & Rha & Fuc & Ara & Xyl & Man & Gal & Glc & UA & Total & Pectin & Protein & & \\
\hline \multicolumn{14}{|l|}{ Orange bagasse } \\
\hline OB1 & $1.7 \pm 0.0$ & $0.4 \pm 0.0$ & $9.3 \pm 0.0$ & $2.6 \pm 0.0$ & $2.7 \pm 0.0$ & $7.7 \pm 0.0$ & $20.0 \pm 0.1$ & $14.9 \pm 0.7$ & $59.4 \pm 0.9$ & 33.6 & $5.4 \pm 0.0$ & $93 \pm 1.0$ & $26 \pm 0.5$ \\
\hline OB2 & $1.2 \pm 0.0$ & $0.4 \pm 0.1$ & $7.4 \pm 0.1$ & $2.2 \pm 0.1$ & $2.4 \pm 0.0$ & $5.9 \pm 0.2$ & $16.7 \pm 0.2$ & $16.3 \pm 1.2$ & $52.5 \pm 0.7$ & 30.8 & $8.3 \pm 0.1$ & $92 \pm 1.5$ & $17 \pm 0.7$ \\
\hline \multicolumn{14}{|c|}{ Passion fruit peel } \\
\hline PFP1 & $0.4 \pm 0.0$ & $0.4 \pm 0.0$ & $1.8 \pm 0.1$ & $10.6 \pm 0.9$ & $3.2 \pm 0.0$ & $2.5 \pm 0.1$ & $28.9 \pm 0.2$ & $15.1 \pm 0.9$ & $63.0 \pm 0.1$ & 19.8 & $7.1 \pm 0.1$ & $28 \pm 1.1$ & $65 \pm 3.9$ \\
\hline PFP2 & $0.4 \pm 0.0$ & $0.5 \pm 0.0$ & $2.0 \pm 0.0$ & $7.8 \pm 0.3$ & $3.9 \pm 0.2$ & $2.7 \pm 0.0$ & $31.1 \pm 1.2$ & $18.3 \pm 0.8$ & $66.8 \pm 2.5$ & 23.4 & $6.2 \pm 0.0$ & $29 \pm 0.7$ & $50 \pm 5.1$ \\
\hline
\end{tabular}

Rha: rhamnose, Fuc: fucose, Ara: arabinose, Xyl: xylose, Man: mannose, Gal: Galactose, Glc: glucose, UA: uronic acids; DM: degree of methylation (moles of methanol per 100 moles of galacturonic acid residues); DA: degree of acetylation (moles of acetic acid per 100 moles of galacturonic acid residues); Pectin: sum of UA, Rha, Ara and Gal.

Inulin from chicory root was provided by its manufacturer (Orafti, Amsterdam, the Netherlands) and was used as positive control (purity $\geq 90 \%$, degree of polymerization $>10$ ). Simulated ileal effluent medium (SIEM) was used as control and is described in one of the following sections.

\section{Fermentation experiments}

\section{In vitro model of the proximal colon (TIM-2)}

TIM-2 mimics the proximal colon and has been described before (9). Shortly, this extensively used and validated dynamic in vitro model simulates the conditions in the colon (temperature kept at $37^{\circ} \mathrm{C}, \mathrm{pH} \mathrm{5.8}$, anaerobic 
environment, removal of microbial metabolites simulating uptake by the body, peristaltic movements) (Supplemental Figure 1 - Adapted from (10)).

\section{Microbiota}

The inoculum used for the TIM-2 experiments was a standardized microbiota prepared from faecal material from 5 healthy individuals ( 3 female and 2 male), age range 20-33 years and body mass index (BMI) $21.69 \pm 0.80 \mathrm{~kg} / \mathrm{m}^{2}$. Participants provided a signed consent prior to donation. The exclusion criteria included the use of prebiotics, probiotics, laxatives and antibiotics in the month prior to donation.

Volunteers were provided with all necessary material for self-collection of their faeces. They were instructed to defaecate in a bag $(300 \mathrm{~mm} \times 500 \mathrm{~mm}, 100 \mu \mathrm{m}$ thick, ZPH Verpakkingen, Zeist, the Netherlands) and immediately place it into a gastight plastic jar containing anaerobic packs (AnaeroGen ${ }^{\mathrm{TM}}$, Oxoid, Cambridge, UK). Faeces were frozen $\left(-80^{\circ} \mathrm{C}\right)$ until standardization.

To standardize the microbiota, faeces were defrosted, mixed in an anaerobic cabinet and prepared as described before (11). The faecal slurry was aliquoted, snap-frozen in liquid nitrogen and stored at $-80{ }^{\circ} \mathrm{C}$. Standardization of microbiota allows the use of the same microbiota for all experiments (permitting comparison among the fermentations of different test compounds) and it was previously demonstrated that it results in a representative microbiota in terms of composition and activity (12).

\section{Simulated ileal effluent medium (SIEM) and dialysis solution}

SIEM simulates the material reaching the colon (Western diet) and it was used as standard feeding, denoted as control (13). It contains indigestible carbohydrates (pectin, xylan, arabinogalactan, amylopectin and starch), protein, vitamins, and bile (13). Tween 80 was omitted because it previously was found to hinder analysis of carbohydrate degradation products (14). The $\mathrm{pH}$ was adjusted to 5.8 to simulate the $\mathrm{pH}$ from the proximal colon.

Dialysis solution contained (per litre): $2.5 \mathrm{~g} \mathrm{~K}_{2} \mathrm{HPO}_{4} .3 \mathrm{H}_{2} \mathrm{O}, 4.5 \mathrm{~g} \mathrm{NaCl}, 0.005 \mathrm{~g}$ $\mathrm{FeSO}_{4} .7 \mathrm{H}_{2} \mathrm{O}, 0.5 \mathrm{~g} \mathrm{MgSO}_{4} .7 \mathrm{H}_{2} \mathrm{O}, 0.45 \mathrm{~g} \mathrm{CaCl}_{2} .2 \mathrm{H}_{2} \mathrm{O}, 0.05 \mathrm{~g}$ bile and $0.4 \mathrm{~g}$ cysteine $\cdot \mathrm{HCl}$, plus $1 \mathrm{~mL}$ of the vitamin mixture; pH 5.8 (Aguirre et al., 2015). All medium components were purchased at Tritium Microbiology (Eindhoven, the Netherlands).

\section{Experimental set up}

In order to create a complete anaerobic environment, TIM-2 was flushed with gaseous nitrogen for 3 hours prior to the addition of the standardized microbiota. Then, approximately $30 \mathrm{~mL}$ of the standardized microbiota was 
added to $90 \mathrm{~mL}$ of dialysis solution (described above) to complete the volume of the system $(120 \mathrm{~mL})$. The microbiota was allowed to adapt to the system for 16 hours while being fed with SIEM $(2.5 \mathrm{~mL} /$ hour) (denoted as "adaptation period", Figure 1). Next, the feeding system was stopped for 2 hours (denoted as "starvation period"), in which the microbiota fermented the remaining carbohydrates from SIEM. Subsequently, samples were collected (referred to as tOh) and the system was fed with SIEM preparation containing the test compounds instead of the standard carbohydrates $(7.5 \mathrm{~g} /$ day).

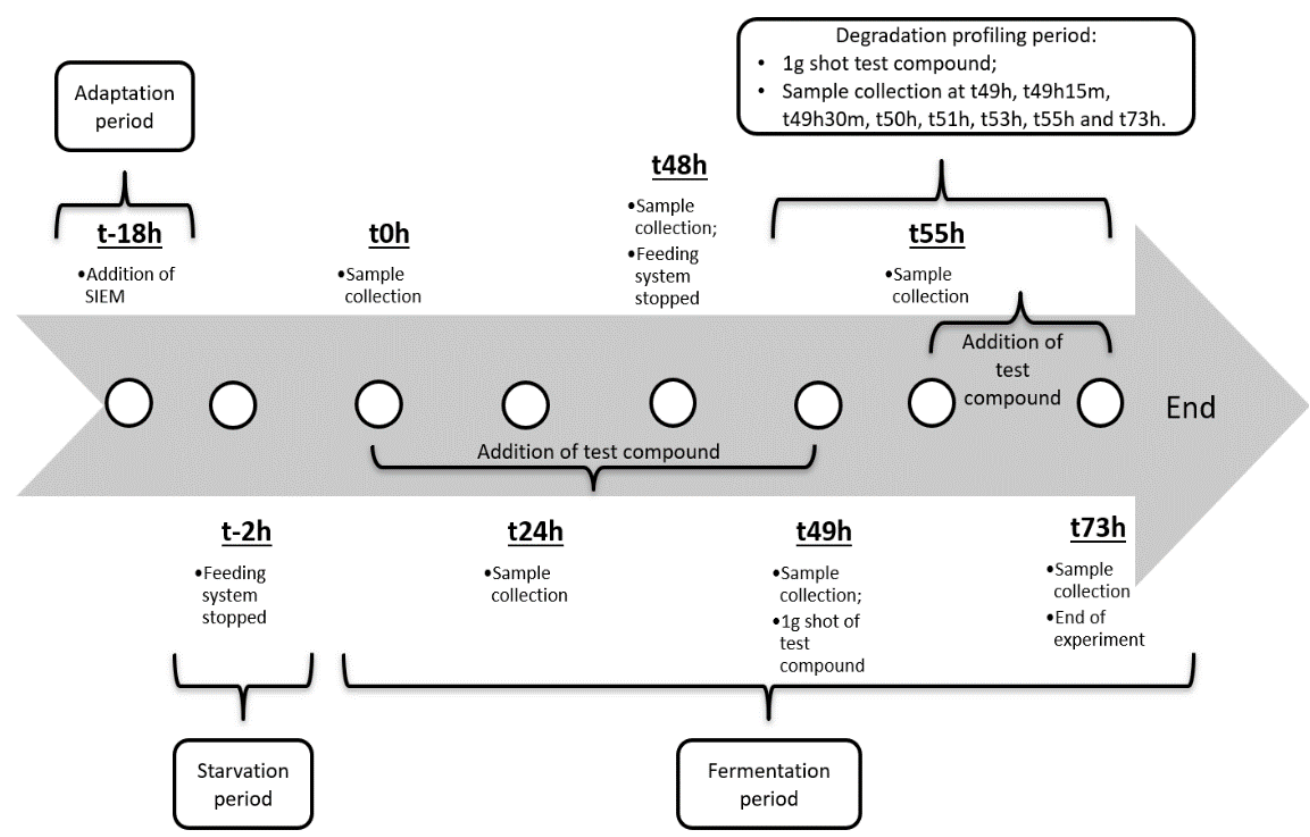

Figure 1: Schematic figure of the experimental set up of fermentation experiments in TIM-2.

Samples from lumen and dialysate out were collected every $\sim 24$ hours $(\mathrm{t} 0 \mathrm{~h}$, $\mathrm{t} 24 \mathrm{~h}, \mathrm{t} 48 \mathrm{~h}$ and $\mathrm{t} 73 \mathrm{~h}$ ). After taking samples at $\mathrm{t} 48 \mathrm{~h}$, the feeding system was stopped for 1 hour. At t49h, samples were collected and a shot of $1 \mathrm{~g}$ of test compound diluted in dialysate $(1: 15)$ was added to the system. Samples were subsequently collected at $\mathrm{t} 49 \mathrm{~h} 15 \mathrm{~m}, \mathrm{t} 49 \mathrm{~h} 30 \mathrm{~m}, \mathrm{t} 50 \mathrm{~h}, \mathrm{t} 51 \mathrm{~h}, \mathrm{t} 53 \mathrm{~h}, \mathrm{t} 55 \mathrm{~h}$ and $\mathrm{t} 73 \mathrm{~h}$ for profiling the degradation of the fibres (constituent monosaccharide analysis). The feeding system was reconnected again after t55h. The experimental scheme can be seen in Figure 1.

\section{SCFA, BCFA and organic acids analyses}

Samples from fermentation experiments in TIM-2 (lumen and dialysate - t0h, $\mathrm{t} 24 \mathrm{~h}, \mathrm{t} 48 \mathrm{~h}$ and $\mathrm{t} 73 \mathrm{~h}$ ) were analyzed to quantify SCFA (acetate, propionate and 
butyrate), BCFA (iso-butyrate and iso-valerate), as well as organic acids (caproate, lactate, succinate and formate) by Brightlabs (Venlo, the Netherlands).

Samples were centrifuged at $14.000 \mathrm{rpm}$ for 10 minutes, and the supernatant was filtered through a $0.45 \mu \mathrm{m}$ PTFE filter and diluted with mobile phase (lumen 1:5 and dialysate 1:2). The samples were analysed using ion exclusion chromatography (IEC) on an 883 Ion Chromatograph (IC; Metrohm, Switzerland), using a Transgenomic IC Sep ICE-ION-300 column (30 cm length, $7.8 \mathrm{~mm}$ diameter and $7 \mu \mathrm{m}$ particles) and a MetroSep RP2 Guard. The mobile phase consists of $1.5 \mathrm{mM}$ aqueous sulphuric acid. A flowrate of $0.4 \mathrm{ml} / \mathrm{min}$ was used. The temperature of the column was $65{ }^{\circ} \mathrm{C}$. The organic acids were detected using suppressed conductivity detection. Ten microliters were loaded on the column by an autosampler 730 (Metrohm). Molecules were eluted according to their pKa.

\section{Microbiota composition}

Lumen samples from TIM-2 (t0h and $773 \mathrm{~h}$ ) were subjected to DNA sequencing for microbial composition analysis. In brief, genomic DNA isolation (ZYMO Research Co., CA, USA), PCR amplification of the 16S rRNA gene (V3-V4 region), barcoding and library preparation were performed by BaseClear (Leiden, the Netherlands). Short paired-end sequence reads were generated using the Illumina MiSeq system and converted into FASTQ files using the BCL2FASTQ pipeline version 1.8.3. Quality trimming was applied based on Phred quality scores.

\section{Constituent monosaccharide composition of lumen samples}

Lumen samples (t49h, t49h15m, t49h30m, t50h, t51h, t53h, t55h and $\mathrm{t} 73 \mathrm{~h}$ ) were heated at $100{ }^{\circ} \mathrm{C}$ for 5 minutes to inactivate bacteria and enzymes. The samples were then freeze-dried (total digest).

Part of the freeze-dried samples was sequentially suspended in Millipore water $(10 \mathrm{mg} / \mathrm{mL})$ and centrifuged $\left(4{ }^{\circ} \mathrm{C}, 10 \mathrm{~min}, 4000 \mathrm{rpm}\right)$ to extract the soluble fraction. The insoluble part was freeze dried for analysis (insoluble digest).

Monosaccharide constituent composition was measured by derivatizing the sugars into alditol acetates as previously described (15). The total uronic acid content was determined with the automated meta-hydroxydiphenyl assay (16). Galacturonic acid (12.5-100 $\mu \mathrm{g} / \mathrm{mL}$ ) was used for calibration. Pectin was calculated as the total of uronic acids (UA), rhamnose (Rha), arabinose (Ara) and galactose (Gal) (w/w \%). 
Constituent monosaccharide present in the soluble fraction was calculated from the difference in individual monosaccharide contents present in the total digest and the insoluble digest.

\section{Data analyses}

The experiments were performed in duplicate $(\mathrm{n}=2)$ per test compound, except for control $(n=6)$, which was run along with the other test compound in each week of experimenting, to show reproducibility throughout time.

QIIME software package (version 1.9.0) was used for microbial analyses (17). The sequences were classified using Greengenes (version 13.8) as a reference $16 \mathrm{~S}$ rRNA gene database.

Statistical analysis was performed with RStudio (3.1.3) (18). Kruskal-Wallis test was used to test differences in multiple categories, with $p$-values $\mathrm{p}<0.05$ set to be significantly different. Dunn post hoc test was utilized to find differences between groups. Spearman correlation was calculated between the relative abundance of operational taxonomic units (OTUs) and continuous variables. Multiple comparison was corrected using the false discovery rate (FDR), and qvalues (adjusted p-values) were considered significantly different at $<0.05$.

Microbial composition changes induced by test compounds were also assessed through the difference of their initial relative abundance and final abundance (t73h - t0h) and compared to control.

Linear discriminant analysis effect size (LEfSe) (19) was used to find biomarkers between groups using relative abundances from the OTU tables generated in QIIME. Phylogenetic Investigation of Communities by Reconstruction of Unobserved States (PICRUSt) (20) was used to predict the gene families contributing to the metagenomes of the samples identified using $16 \mathrm{~S}$ rRNA sequencing. This data was visualized using statistical analysis of taxonomic and functional profiles (STAMP) (21).

\section{Results and discussion}

\section{Monosaccharide composition of AIS fractions}

The constituent monosaccharide compositions of the four test compounds (AIS fraction) used in TIM-2 fermentations are shown in Table 1, and have been reported previously in detail elsewhere (8).

Briefly, the pectins as dominantly present in the AIS fractions of orange bagasses were highly methyl-esterified, with a degree of methylation (DM) of 93 and 92 for $\mathrm{A}-\mathrm{OB} 1$ and $\mathrm{A}-\mathrm{OB} 2$, respectively. The main monosaccharide present was glucose representing $20 \%$ of the total carbohydrate present for A- 
OB1 and $16.7 \%$ for A-OB2. The two bagasses were very similar in their composition, with the main differences in the protein content $(5.4 \%$ and $8.3 \%)$ and degree of acetylation (DA) $(26 \%$ and $17 \%$ for A-OB1 and A-OB2, respectively).

In contrast, pectins as present in the isolated AIS fibres from passion fruit peels were low methyl-esterified ( $\mathrm{DM} \leq 50)$. Additionally, the amount of pectin present $(20-23 \% \mathrm{w} / \mathrm{w})$ was lower than their citrus counterpart. The two samples of A-PFPs had glucose as the main monosaccharide $(29-31 \% \mathrm{w} / \mathrm{w})$, followed by uronic acid (15-18\% w/w) and xylose (8-11\% w/w) (Table 1).

\section{Microbial activity}

\section{Production of SCFA and organic acids}

Host diet composition and food intake pattern are important determinants for metabolites produced by the gut microbiota (22). SCFA constitute the major bacterial end products formed from fermentation of indigestible foods (23). They exert beneficial roles on the host, and thus, a food component that can have a positive effect on the production of these metabolites is desirable.

Table 2: Average ( \pm range) cumulative production of SCFA (acetate, propionate and butyrate), BCFA (iso-butyrate and iso-valerate) and other organic acids (succinate, lactate, formate and caproate) by the gut microbiota on the standard medium and inulin, and the substrates tested after 73 hours fermentation (mmol).

\begin{tabular}{|c|c|c|c|c|c|c|}
\hline & Control & Inulin & A-0B1 & A-0B2 & A-PFP1 & A-PFP2 \\
\hline Acetate & $65.5 \pm 7.7$ & $53.6 \pm 0.8$ & $53.4 \pm 3.8$ & $63.8 \pm 2.1$ & $42.0 \pm 12.2$ & $52.9 \pm 2.6$ \\
\hline Propionate & $14.2 \pm 5.2$ & $11.9 \pm 0.8$ & $12.9 \pm 0.1$ & $24.9 \pm 0.3$ & $21.4 \pm 0.9$ & $26.4 \pm 1.1$ \\
\hline Butyrate & $53.4 \pm 3.6$ & $59.0 \pm 7.7$ & $15.4 \pm 0.0$ & $29.6 \pm 0.5$ & $20.3 \pm 1.3$ & $21.3 \pm 0.4$ \\
\hline Total SCFA & $133.2 \pm 10.7$ & $124.6 \pm 7.6$ & $81.8 \pm 3.6$ & $118.3 \pm 1.3$ & $83.7 \pm 14.4$ & $100.7 \pm 3.3$ \\
\hline Lactate & $1.0 \pm 0.7$ & $0.6 \pm 0.4$ & $\mathrm{NG}$ & $0.7 \pm 0.7$ & $\mathrm{NG}$ & $\mathrm{NG}$ \\
\hline Succinate & $18.4 \pm 2.4$ & $26.5 \pm 3.9$ & $2.9 \pm 0.5$ & $6.6 \pm 4.4$ & NG & $\mathrm{NG}$ \\
\hline Formate & $1.9 \pm 1.4$ & $2.9 \pm 0.2$ & $1.1 \pm 0.7$ & $5.1 \pm 2.5$ & $3.0 \pm 0.4$ & $0.9 \pm 0.4$ \\
\hline Caproate & $\mathrm{NG}$ & NG & NG & NG & $0.1 \pm 0.1$ & $\mathrm{NG}$ \\
\hline $\begin{array}{r}\text { Total Org. } \\
\text { Acids }\end{array}$ & $21.3 \pm 2.4$ & $30.0 \pm 4.1$ & $4.0 \pm 1.1$ & $12.4 \pm 6.2$ & $3.1 \pm 0.8$ & $0.9 \pm 0.4$ \\
\hline Iso-butyrate & $0.5 \pm 1.0$ & $0.1 \pm 0.1$ & $\mathrm{NG}$ & $0.6 \pm 0.4$ & $1.1 \pm 0.1$ & $1.2 \pm 0.0$ \\
\hline Iso-valerate & $0.8 \pm 0.7$ & $0.2 \pm 0.1$ & NG & $1.9 \pm 0.7$ & $1.3 \pm 0.1$ & $1.8 \pm 0.7$ \\
\hline Total BCFA & $1.3 \pm 1.4$ & $0.3 \pm 0.1$ & $N G$ & $2.5 \pm 1.1$ & $2.4 \pm 0.2$ & $3.0 \pm 0.7$ \\
\hline
\end{tabular}

NG: below detection limit $(\leq 0.05)$ 
Fermentation of A-OB1 resulted in the lowest total cumulative amount of SCFA among all test compounds (81.8 mmol) (Table 2). On the other hand, A-OB2 fermentation showed a similar total amount of SCFA (118.3 mmol) as inulin (124.6 mmol), our positive control. A-PFP1 fermentation produced in total 83.7 mmol of SCFA and A-PFP2 $100.7 \mathrm{mmol}$.

Over time, A-OB1 fermentation mainly increased the proportion of propionate, which increased in ratio from $9.7 \%$ (at $\mathrm{t} 24 \mathrm{~h}$ ) to $15.8 \%$ after 73 hours experimental period (Figure 2).

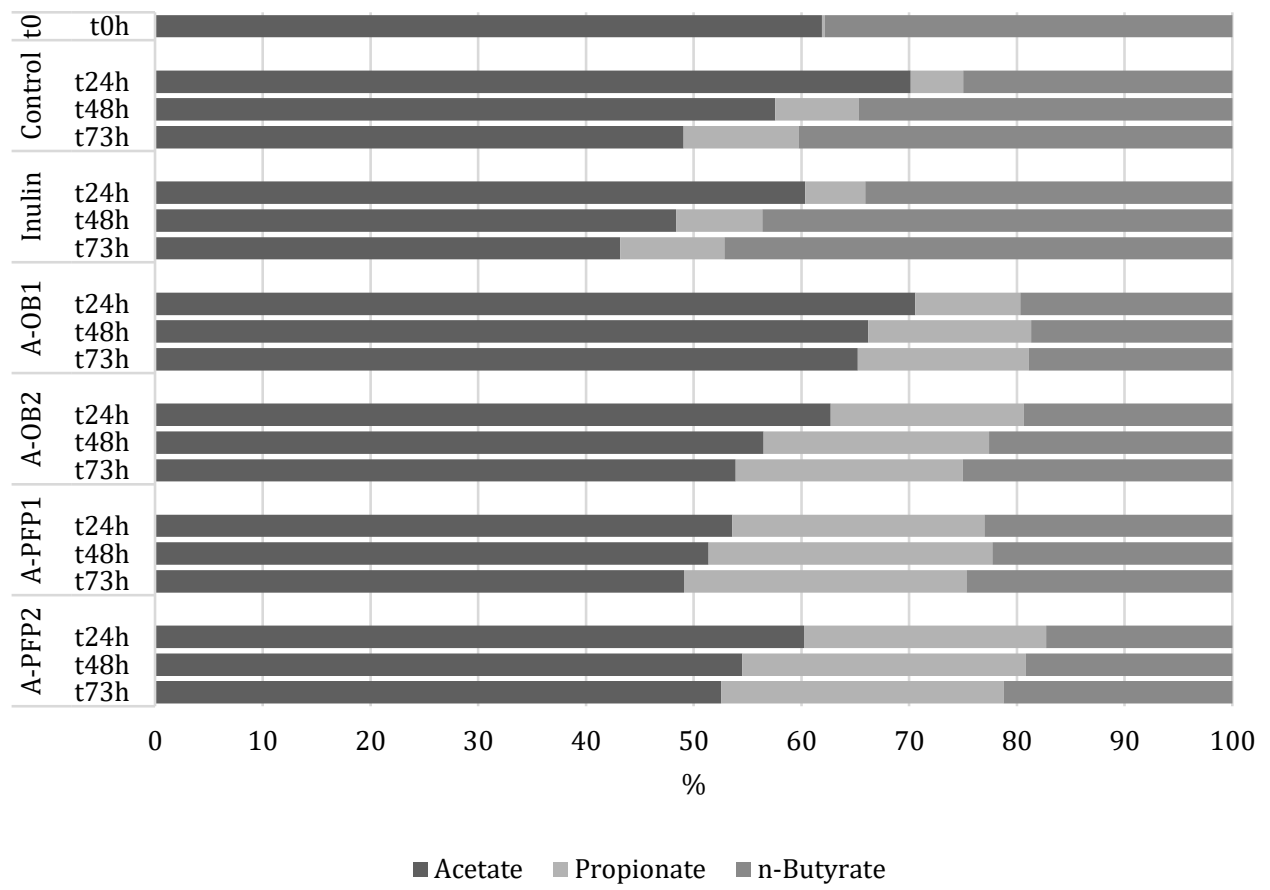

Figure 2: Average ratio of SCFA produced during fermentation of the different test compounds. T0 represents the average ratio of SCFA from of all runs with test compounds.

Compared to A-OB1, fermentation of A-OB2 resulted in a different profile regarding the production of bacterial metabolites. Fermentation of A-OB2 increased the proportion of butyrate over time $19.4 \%$ at $\mathrm{t} 24 \mathrm{~h}$ and $25 \%$ at t73h) (Figure 2). It did not show, however, a pronounced butyrogenic effect like the positive control inulin, where the relative proportion of butyrate increased from $34.1 \%$ at $\mathrm{t} 24 \mathrm{~h}$ to $47.1 \%$ at $\mathrm{t} 73 \mathrm{~h}$ ). The importance of butyrate for host health is diverse. Butyrate is the main fuel for colonocytes (23) and contributes to the conservation of the mucus barrier through the up-regulation of mucinencoding gene expression, assisting therefore, in maintaining a healthy 
epithelial layer in the colon. It has anti-inflammatory effects and induces apoptosis in in vitro cultured colon cancer cells (24), and thus, might have anticarcinogenic effects.

Generation of butyrate upon fermentation of passion fruit peels was similar to that of orange bagasses, but lower than the standard medium and inulin controls (Table 2). A study performed with mice which had received standard diet enriched with passion fruit peel for fifteen days revealed an increase in faecal acetate and butyrate when compared to the control group which received standard diet (25). The aforementioned study used dried passion fruit peel, which contained not only the fibre component, but also polyphenols for instance. In our study, the isolated fibre material from passion fruit peels (AIS) without the phenolic compounds was used. Polyphenols are also known to exert an effect on the gut microbiota (26), which might explain the differences found regarding the effects on acetate and butyrate production.

Although A-OB1 stimulated the production of propionate over time, it did not provide a low ratio between acetate to propionate as A-OB2 did (4.1 and 2.6 for $\mathrm{A}-\mathrm{OB} 1$ and $\mathrm{A}-\mathrm{OB} 2$, respectively). Studies are indicating that low ratios of acetate to propionate have hypolipidemic effects on the host (27-29). Acetate stimulates the synthesis of lipids, while propionate counteracts de novo lipogenesis from acetate (30), demonstrating the importance of the SCFA product ratios. A-PFP1 and A-PFP2 resulted in similar ratios of acetate to propionate (1.9 and 2.0, respectively), with higher percentages of propionate than for A-OB.

Lactate and succinate are intermediate metabolites in intestinal microbiology and are usually present in lower amounts because they are consumed by other microbes through cross-feeding that convert them into SCFA, such as propionate and butyrate $(23,31)$. Their accumulation is an indication that the substrate was fermented fast (31). Control and inulin were the substrates that resulted in accumulation of mainly succinate, but also lactate (Table 2). In contrast, passion fruit peels resulted mostly in negligible amounts of these intermediate metabolites, probably because these substrates were consumed at a slow pace. Slow fermentation has a positive effect, since a substrate that is fermented slowly has more chances to reach the distal part of the colon, to be fermented there. Most carbohydrates are usually fermented extensively in the proximal colon (6). As a consequence, the microbiota in the distal colon ferment proteins, producing toxic metabolites which have been shown to have direct effects on colon cancer incidence $(32,33)$. Fermentation of A-PFP1 resulted in the highest amount of formate $(3.0 \mathrm{mmol})$ whilst A-PFP2 generated the smallest amount $(0.9 \mathrm{mmol})$ among all test compounds (Table 2$)$. Generation of 
caproate was negligible in all test compounds, apart from A-PFP1 that still produced a small amount $(0.1 \mathrm{mmol})$ after 73 hours fermentation. The role of these metabolites in health and disease is unclear.

Taken together, analyses of bacterial activity revealed that regarding SCFA and organic acids production, $\mathrm{A}-\mathrm{OB} 2$ was the test component that showed the most positive profile. The total cumulative amount of SCFA was similar to inulin, our positive control. SCFA are essential to maintain a lower $\mathrm{pH}$ in the colon, which is a selective mechanism to favour the growth of beneficial bacteria over pathogens (34). Although the generation of butyrate was smaller than inulin and control, still, A-OB2 produced higher amounts of butyrate compared to the other test compounds. Additionally, the ratio of acetate to propionate was lower than control, inulin and A-OB1, indicating that intervention with A-OB2 might be useful to promote the production of SCFA, and consequently, contribute to lipids lowering in hyperlipidaemic individuals. It is important to highlight that although passion fruit peels (A-PFP1 and A-PFP-2) resulted in lower cumulative SCFA production, generation of negligible amounts of lactate and succinate indicated that they were fermented at a slow pace, a positive characteristic when considering health effects in the distal colon, e.g., against colon cancer. In this sense, passion fruit peels could be used together with other dietary fibres with the main objective to serve as source of energy for bacteria residing in the distal colon, and consequently protecting this segment against health problems that commonly affect the distal colon in Western society.

\section{Production of BCFA}

The total cumulative production of BCFA (iso-butyrate and iso-valerate) was negligible upon A-OB1 administration ( $\leq 0.01 \mathrm{mmol}$ ), while it was $2.5 \mathrm{mmol}$ on A-OB2 (Table 2). Notably, A-PFP2 fermentation resulted in the highest amount of BCFA generated $(3.0 \mathrm{mmol})$, despite not being the test compound with the highest amount of protein content (6.2\%) (Table 1). This is likely reflecting the types of amino acids that make up the proteins of A-PFP2 (not analysed here). Studies have shown that once in the colon, fermentation of peptides and amino acids produces not only SCFA, but also BCFA (23) and other potentially toxic substances such as ammonia, which have negative health effects (35). A similar study showed a higher production of BCFA by microbiota from lean individuals upon apple fibre and sugar beet pectin administration $(4.5 \mathrm{mmol}$ and $4.1 \mathrm{mmol}$, respectively), when compared to our results (36). On the other hand, when a microbiota originating from obese individuals was used, a lower generation of BCFA was found for the identical substrates $(2.6 \mathrm{mmol}$ on apple fibre and 1.4 mmol on sugar beet pectin). Another study performed in TIM-2 with a 
microbiota originating from healthy individuals and using mango peel as a substrate also showed a higher generation of BCFA ( $>4.0 \mathrm{mmol}$ ) (37) than the test compounds used in the current study. Importantly, the negative effects of proteolytic fermentation might be suppressed through an adequate ingestion of non-digestible fermentable carbohydrates, which are the preferred substrate for microbial fermentation, leaving amino acids and peptides to be used for microbial biomass formation instead of being used for energy production $(24,31)$. The differences in the formation of BCFA were therefore dependent of the type of substrate used. Compared to other types of fibres (e.g. apple fibre, sugar beet pectin and mango peel), fermentation of orange bagasses and passion fruit peels resulted in similar or even lower amounts of BCFA. The results found here reinforce the fact that microbial metabolism is highly affected by the type of substrate that reaches the colon. The gut microbiota can be manipulated by diverse types of fibres with the goal to achieve a healthier profile and metabolism that in turn will bring benefits to the host.

\section{Assessment of microbiota composition}

\section{Principal Coordinate Analysis (PCoA) and Linear discriminant analysis effect size (LEfSe)}

In order to identify which factors could be associated with shaping of bacterial communities after 73 hours fermentation, Principal Coordinate Analysis (PCoA) was performed. The unweighted PCoA plot (Figure 3-B) shows that samples were clustered according to their substrate.
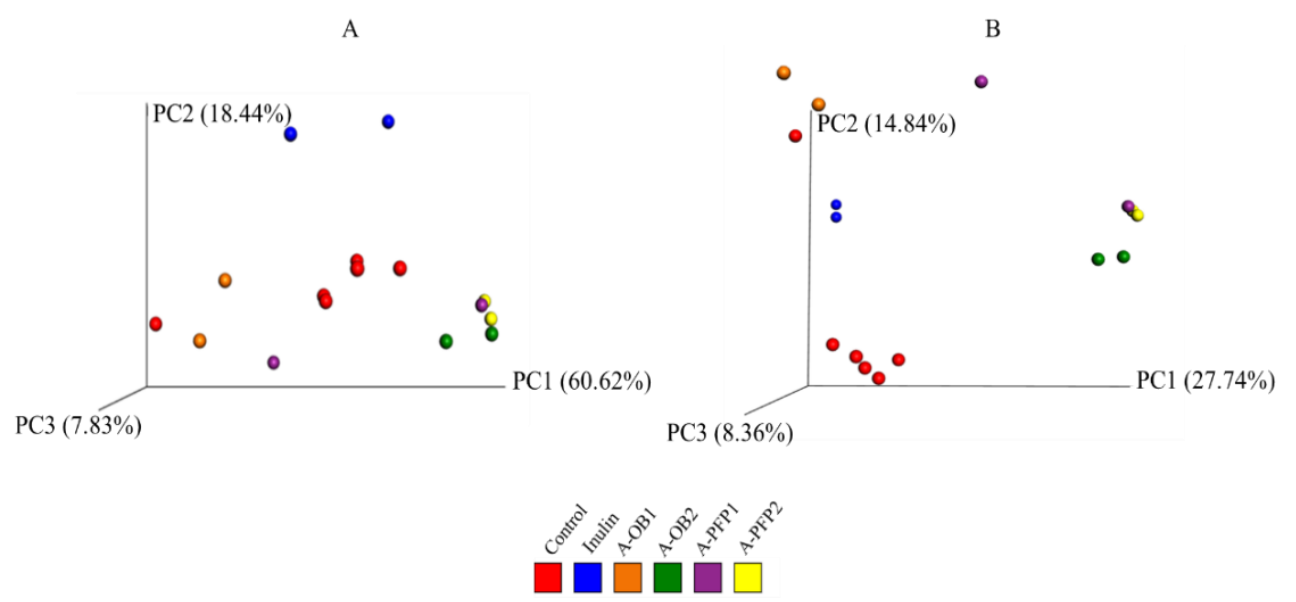

Figure 3: Principal Coordinate Analysis (PCoA) plots of the microbiota after 73 hours fermentation in TIM-2 with Weighted (A) and Unweighted (B) UniFrac. Samples are plotted on the first three Principal Coordinates (PC1, PC2 and PC3). 
Orange bagasses had a distinct separation along the Principal Coordinate 1 (PC1). PC1 explained $60.62 \%$ of variation between the samples in weighted UniFrac and $27.74 \%$ in unweighted UniFrac (Figure 3-A and B). To visualize the taxa driving the differences in the PCoA plot, a boxplot (and corresponding heatmap) was generated in QIIME for the top 10 Operational Taxonomic Units (OTUs), which showed that A-OB1 clustering was mainly influenced by the genus Collinsella (Supplemental Figure 2). However, as indicated in Supplemental Figure 2, it was not entirely unique to A-OB1 and could also not be confirmed by LEfSe (Supplemental Figure 3), which showed that Prevotella is a discriminating feature for this substrate. A-OB2 clustering had the genus Ruminococcus as the main driver in the boxplot (and heatmap; Supplemental Figure 2). So, despite similar monosaccharide composition, these two bagasses stimulate different microbes, indicating some (structural) differences in the polysaccharides present (see below). LEfSe (Supplemental Figure 3) and the boxplot (Supplemental Figure 2) show another Ruminococcus OTU and Catenibacterium as clear discriminants for inulin.

Different from the orange bagasses, the two batches of passion fruit peel had a more similar effect, not only on the production of bacterial metabolites, as described above, but also on the microbiota composition. This similarity can be observed in Figure 3, where the PCoA plots showed that passion fruit peel samples clustered together (although one duplicate of A-PFP1 looks like an outlier). Similar to A-OB2, the same enriched OTU within the genus Ruminococcus was the main driver for this clustering.

PICRUSt was used to predict the microbial pathways contributing to the metagenomes of the samples identified based on 16S rRNA sequencing, projection on the KEGG database, and data visualization using STAMP. Using Welsh's two-sided t-test with Bonferroni correction, pathways predicted for each substrate were compared to the other food by-products (e.g., A-OB1 compared to A-OB2+the two A-PFPs, etc.). All significant predicted pathways projected on the KEGG pathways are indicated in Supplemental Table 1. The highest number of significantly different predicted pathways was seen for APFP2 (25), followed by A-OB1 (19). A number of pathways clearly related to microbial metabolism have been plotted as box-plots (Supplemental Figure 3BF). For A-OB1 the pathway 'protein digestion and absorption' was predicted to be higher than in the other substrates (Supplemental Figure 3B). How this relates to the negligible BCFA production is unclear. Perhaps the digested and absorbed protein was exclusively incorporated in microbial biomass rather than fermented. For A-PFP2 several pathways related to microbial activity were higher than in the other samples. These included butyrate (butanoate; 
Supplemental Figure 3C) and propionate (propanoate; Supplemental Figure 3E) metabolism, general metabolism (TCA cycle; Supplemental Figure 3F), but also pyruvate metabolism, fatty acid metabolism, and energy metabolism; Supplemental Table 1), and methane metabolism (Supplemental Figure 3D). Cumulative propionate production was indeed highest on this substrate, but this was not the case for butyrate (Table 2). Whether or not methane was produced under these conditions was not tested. Multiple group comparison using the Kruskal-Wallis test did not show discriminative pathways, but this is likely due to the small dataset.

\section{Changes in microbiota composition over time}

A-OB2 fermentation in TIM-2 resulted in a more diverse microbiota composition after 73 hours fermentation, when compared to A-OB1 and to all other test compounds (Supplemental Figure 4). Shannon index (Supplemental Figure 4A) and "observed OTUs" (Supplemental Figure 4B) revealed that A-OB2 was the substrate which stimulated the growth of more bacterial species (either abundance or evenness), whereas A-OB1 and inulin presented the lowest Shannon index and observed OTUs. Low microbial diversity is commonly correlated with health problems $(38,39)$. A study made with 292 obese and non-obese Danish individuals showed that according to the number of microbial genes present in faecal samples, this population could be classified as "low gene counts" (LGC; low diversity) and "high gene counts" (HGC; high diversity) (40). HGC individuals had a higher prevalence of anti-inflammatory species, like Faecalibacterium prausnitzii, as well as Akkermansia and Bifidobacterium, whereas LGC individuals harboured more potentially proinflammatory bacteria, such as Bacteroides and Ruminococcus gnavus, commonly associated with IBD (40). Importantly, the LGC group was composed of a significantly higher proportion of obese participants with metabolic disturbances that could put them at increased risk for T2D and cardiovascular disorders (40).

The effects that each test compound had on the microbiota composition, when compared to the initial relative abundance (at $\mathrm{t} 0 \mathrm{~h}$ ) and against control, is displayed in Figure 4. Although the relative abundance of Bifidobacterium decreased upon A-OB1 fermentation, it decreased less than the control, while on A-OB2 it decreased more than the control. On average, the relative abundance of the Bifidobacterium genus at the end of the experiment was $40.5 \%$ on $\mathrm{A}-\mathrm{OB} 1$ and $6 \%$ on A-OB2 (Figure 5). Fermentation of both passion fruit peels also decreased the relative abundance of Bifidobacterium when compared to the control, with A-PFP2 decreasing more than A-PFP1 (Figure 4). 
At the end of the experiment, $20.7 \%$ of the genera on A-PFP1 belonged to Bifidobacterium group, while on A-PFP2 this was 7.3\% (Figure 5). Members of the Bifidobacterium genus produce mainly acetate and lactate from mono- and oligosaccharides (41). This group, therefore, commonly participates in crossfeeding with other genera that are butyrate producers (such as members from the families Lachnospiraceae and Ruminococcaceae) that release mono- and oligosaccharides from complex carbohydrates degradations (42).

\begin{tabular}{|c|c|c|c|c|c|}
\hline OTU ID & Inulin & A-OB1 & A-OB2 & A-PFP1 & A-PFP2 \\
\hline$f \_$Bifidobacteriaceae; $g$ _Bifidobacterium & & * & & & \\
\hline$f_{\ldots}$ Coriobacteriaceae; $g_{\text {_ }}$ & & & & & \\
\hline$f \_$Coriobacteriaceae; $g$ _Collinsella & & & & & \\
\hline$f \_$_Bacteroidaceae;g_Bacteroides & & & & & \\
\hline$f \_$Prevotellaceae;g_Prevotella & & & & & \\
\hline$f \_$Enterococcaceae;g_Enterococcus & & & & & \\
\hline o_Clostridiales; $f$ _ $g$ & & & & & \\
\hline f_Clostridiaceae; $g \_C l o s t r i d i u m$ & & & $*$ & & $*$ \\
\hline$f \_$Lachnospiraceae; Other & & & & & \\
\hline f_Lachnospiraceae;g__ & & & & & \\
\hline f_Lachnospiraceae;g__Blautia & & & & & \\
\hline f_Lachnospiraceae;g_Lachnospira & & & & & \\
\hline f_Lachnospiraceae;g_Roseburia & & & & & \\
\hline$f \_$Ruminococcaceae;g_ & & & & & \\
\hline$f \_$Ruminococcaceae;g_Faecalibacterium & & & & & \\
\hline$f \_$Ruminococcaceae;g_Oscillospira & & & & & \\
\hline$f \_$Ruminococcaceae;g_Ruminococcus & & & & & \\
\hline f_Veillonellaceae;g_Dialister & & & & & \\
\hline$f \quad$ Enterobacteriaceae; $g$ & & & & & \\
\hline
\end{tabular}

* These genera had a lower decrease when compared to the control, but did not increased over time.

\begin{tabular}{|c|l|}
\hline $15 \%$ to $50 \%$ & High increase \\
\hline $5 \%$ to $15 \%$ & Moderate increase \\
\hline $1 \%$ to $5 \%$ & Small increase \\
\hline$-1 \%$ to $1 \%$ & No changes \\
\hline$-1 \%$ to $-5 \%$ & Small decrease \\
\hline$-5 \%$ to $-15 \%$ & Moderate decrease \\
\hline$-15 \%$ to $-50 \%$ & High decrease \\
\hline
\end{tabular}

Figure 4: Heatmap of percentage of changes of bacterial taxa after 73 hours fermentation, compared to control. Green colours represent an increase in abundance, whereas red colours represent a decrease.

Bifidobacterium is used as biomarker for intestinal health because this genus uses primarily carbohydrates as substrate for fermentation, as well as there are no known pathogens in this group (43).

Both orange bagasses had a positive effect on the relative abundance of Collinsella, increasing by $8.3 \%$ and $1.0 \%$ for A-OB1 and A-OB2, respectively. Collinsella is able to ferment a vast range of carbohydrates and deconjugate bile acids (41). The final relative abundance of this genus was $11.7 \%$ on A-OB1 and 
$4.5 \%$ on A-OB2 (Figure 5). A-PFP1 and A-PFP2 caused a diverse effect on the abundance of Collinsella. While A-PFP1 induced a moderate increase $(6.5 \%)$ in this genus, A-PFP2 actually decreased by $1.9 \%$ the abundance of Collinsella. The final abundance was $9.9 \%$ on A-PFP1 and $1.6 \%$ on A-PFP2.

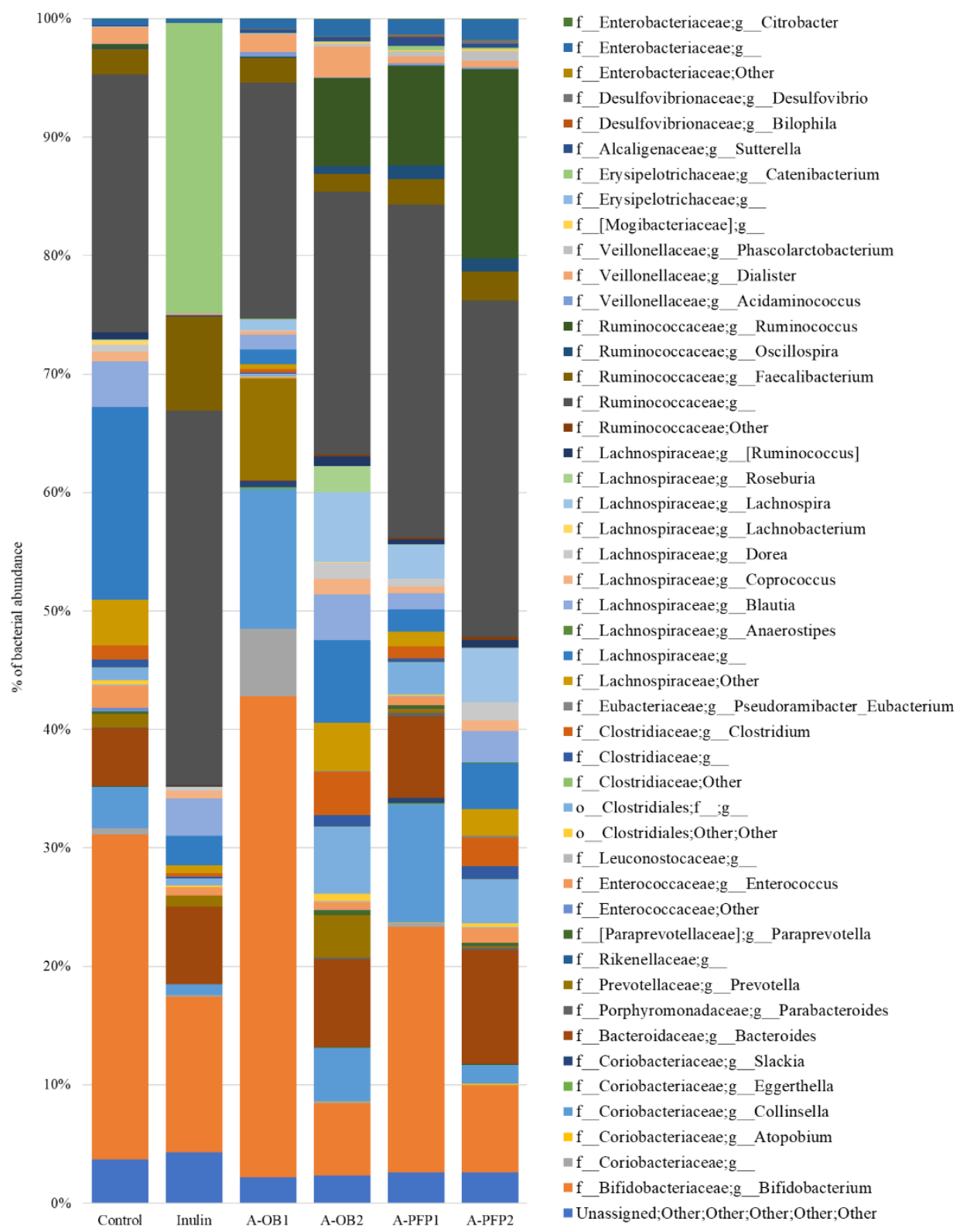

Figure 5: Microbiota composition after 73 hours fermentation with the different test compounds (\% of bacterial abundance). 
A-OB1 and A-OB2 fermentation had different effects on abundance of the genus Bacteroides, resulting in a small decrease $(\sim 5 \%)$, or increase $(\sim 2 \%)$, respectively.

At the end of the experimental period, the relative abundance of Bacteroides on A-OB2 was $7.4 \%$, while on A-OB1 this was only $0.05 \%$ (Figure 5). Passion fruit peels slightly stimulated (1\% to $5 \%$ ) the relative abundance of the genera Bacteroides and Lachnospira. Growth with A-PFP1 and A-PFP2 at the end of the 73 hours fermentation period resulted in an average relative abundance of the Bacteroides and Lachnospira genera of $6.9 \%$ and $9.6 \%$, and $2.8 \%$ and $4.5 \%$, respectively. Spearman correlation between BCFA and OTUs revealed that Bacteroides was positively correlated with iso-butyrate (rho=0.71, qvalue $=0.03$ ), indicating an association among the three test compounds that generated higher amounts of this BCFA (A-OB2, A-PFP1 and A-PFP2), BCFA and Bacteroides. Members of the Bacteroides genus, known as "generalists", possess a broad metabolic potential, being responsible for degrading either proteins or complex glycans (41). They have a great repertoire of carbohydrate-active enzymes (CAZymes) encoded by the polysaccharide utilization loci (PULs) (44). This characteristic gives them the ability to use a plethora of polysaccharides and therefore the advantage of fermenting different substrates that reach the colon (45). An in vitro study in the same TIM-2 model revealed that Bacteroides was enriched upon high protein feeding, with higher production of BCFA when compared to a control and high carbohydrate feeding (46). As Bacteroides species have an important role in peptide and amino acid metabolism, it is not surprising that we found an association with the BCFA iso-butyrate. Isobutyrate production is the result of fermentation of the amino acid valine (47).

The relative abundance of Prevotella increased moderately after $73 \mathrm{~h}$ fermentation with A-OB1 (7.4\%) but showed only a small increase when A-OB2 was administered (2.4\%). Their final abundance was $8.6 \%$ and $3.6 \%$ for A-OB1 and A-OB2, respectively (Figure 5). Abundance of Prevotella on passion fruit peels was negligible $(<1 \%)$. Prevotella uses complex carbohydrates as substrate for fermentation, such as pectin, resistant starch and xylan $(41,48)$, which might explain the higher abundance on A-OB1 that was composed of $33.6 \%$ of pectin (Table 1). However, no association between Prevotella and xylose or passion fruit peels, which were composed of substantial amounts of xylose, were found (Table 1). A comparative study of intestinal microbiota from European and rural African children revealed an enrichment of Prevotella in African children while being completely absent in European children (48). The diet from rural African children was rich in plant polysaccharides, such as 
starch and fibres, and low in fat and animal protein, while the diet of European children was high in animal protein, sugar, starch and fat, and low in fibre (48). Faecal samples from rural African children were higher in SCFA and had higher microbial richness and diversity, which have health-related effects (48).

Blautia decreased by 2.6\% upon A-OB1 fermentation but was not affected its relative abundance when A-OB2 was used as substrate. At t73h its final abundance was $1.3 \%$ and $3.8 \%$ for A-OB1 and A-OB2, respectively (Figure 5). Blautia relative abundance also decreased upon administration of passion fruit peels (from 1\% to 5\%). Blautia is one of the most abundant groups present in the human gastrointestinal tract and produces acetate from hydrogen and carbon dioxide (41). Here Blautia was found in lower abundance than commonly present in the human intestinal tract.

Lachnospira, Roseburia, Ruminococcus and Dialister genera were enriched only upon fermentation of A-OB2, but not A-OB1. Spearman correlation indicated a positive association between Ruminococcus and iso-butyrate (rho=0.69; qvalue=0.04), and between Ruminococcus and uronic acid (rho=0.87; qvalue $=0.04$ ). Fermentation of A-OB2, which increased moderately the relative abundance of Ruminococcus, was also responsible for production of a higher amount of iso-butyrate when compared to A-OB1. Relative abundance of Ruminococcus after 73 hours fermentation was only $0.05 \%$ on A-0B1, but $7.4 \%$ on A-OB2 (Figure 5). An unassigned genus within the family Ruminococcaceae constituted an important member of the microbiota composition after both AOB1 (19.8\%) and A-OB2 (22.1\%) fermentations. A stronger effect of passion fruit peels was observed on the genus Ruminococcus, which increased by $8.15 \%$ and $15.65 \%$ under A-PFP1 and A-PFP2 administration, respectively. The same unassigned genus of the Ruminococcaceae family was present in large proportion after 73 hours fermentation of both passion fruit peels $(\sim 28 \%)$ (Figure 5). Some species of Ruminococcus (e.g. R. bromii and R. champanellensis) are well recognized by their ability to degrade recalcitrant fibres that reach the colon as insoluble particles thanks to their specialized enzyme systems and capacity to adhere to the substrate (49).

Our results corroborate other studies, where the modulation of microbial composition and metabolism was dependent on the substrate.

Here we found that orange bagasse from different batches differentially affected the fermentation pattern in an in vitro model of the proximal colon. The main structural differences between A-OB1 and A-OB2 were the 
percentage of glucose, total carbohydrates, protein and pectin acetylation levels. The small sample size may have hindered the detection of significant correlation of such characteristics and certain bacterial groups. We conclude that differences in the chemical composition of these two fibres material affected the microbial composition and consequently the metabolites produced.

Even though the fermentation of A-OB2 resulted in higher amounts of BCFA than A-OB1 - which is less desirable, the production of SCFA and lactate was higher on $\mathrm{A}-\mathrm{OB} 2$, indicating that this fibre was more prone to fermentation. It remains to be clarified which chemical aspect was responsible for these differences. Notably, besides the effects of chemical composition of fibres on the modulation of the gut microbiota, their physical form is another dimension that needs to be considered. For instance, different forms of resistant starch were found to be utilized by distinct bacterial groups (7). Other features like fibre matrix and particle size may represent important aspects to be taken into consideration when assessing fibre modulation of the gut microbiota (7). Bifidobacterium, Collinsella, Bacteroides, Prevotella, Blautia, Lachnospira and Ruminococcus were the genera that showed higher differences on orange bagasses after 73 hours fermentation. Although the final bacterial composition after fermentation with A-OB1 presented higher abundance of the beneficial microbes Bifidobacterium and Prevotella, A-OB2 stimulated the growth of beneficial bacteria as well, such as Roseburia, and notably, showed a higher compositional diversity and richness.

Isolated fibres from passion fruit peels presented a more similar chemical composition, reflected in a similar fermentation pattern on both substrates tested. After 73 hours fermentation, the microbial compositions on passion fruit peels were similar, with main differences in the abundance of the genus Bifidobacterium (20.7\% - A-PFP1 and 7.3\% - A-PFP2) and Collinsella (9.9\% - APFP1 and 1.6\% - A-PFP2). A-PFP2, however, produced more SCFA (especially acetate) and BCFA than A-PFP1. They both generated lower amounts of SCFA than orange bagasses, control and inulin.

\section{Degradation profiling}

\section{Constituent monosaccharide composition}

Degradation profiling of a 1 gram shot of fibre of each individual test compound was analysed throughout time through the measurement of the constituent monosaccharide levels of the samples. With orange bagasses, the amount of 
monosaccharides present at $\mathrm{t} 50 \mathrm{~h}$ was higher than at $\mathrm{t} 49 \mathrm{~h} 15 \mathrm{~m}$ and $\mathrm{t} 49 \mathrm{~h} 30 \mathrm{~m}$ (not shown). These fibres were very viscous, and apparently the system needed about $30 \mathrm{~min}$ to homogenise the shot. After 1 hour, the amount of carbohydrates started to decrease, indicating bacterial degradation of fibres. Passion fruit peels, however, presented lower viscosity which in turn facilitated the homogenization by the system. The amount of carbohydrates present in the system decreased immediately after the fibre shot, indicating their bacterial utilization.

Utilization of pectic sugars (rhamnose, arabinose, galactose and uronic acid) present in A-OB1 occurred similarly to A-OB2 (Figure 6A-B). Already after 1 hour of fibre shot it was possible to observe that pectic sugars present on side chains (arabinose and galactose) decreased by $66 \%$ and $47.3 \%$, respectively indicating fast bacterial degradation. After 6 hours from fibre shot, 70.7\% of uronic acid, $58.5 \%$ of galactose, $78.5 \%$ of arabinose and $63.9 \%$ of rhamnose present in A-OB1 were used for bacterial fermentation (Figure 6A). Also in case of A-OB2, uronic acid (84.6\%), galactose (62.1\%), arabinose $(89.2 \%)$ and rhamnose $(50.2 \%)$ were used in large proportions after 6 hours of fibre shot (Figure 6B).

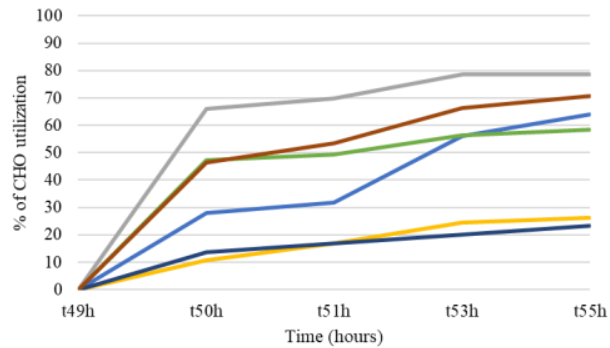

C

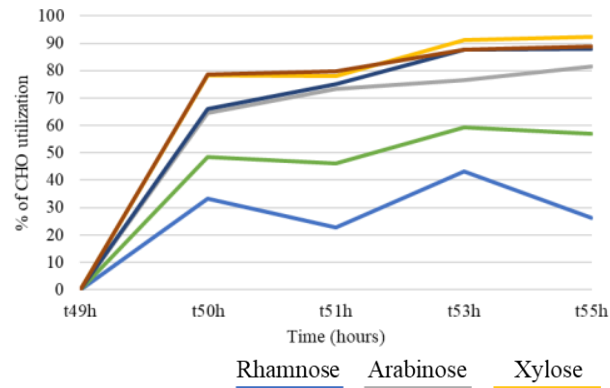

B

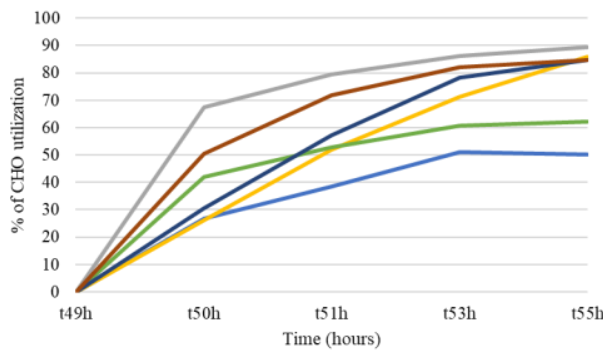

$\mathrm{D}$

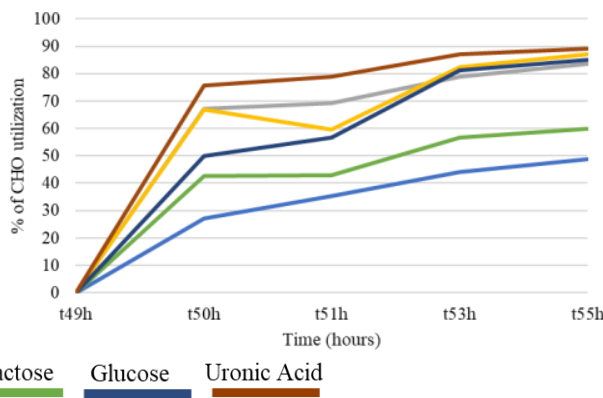

Figure 6: Bacterial degradation of monosaccharides during the fermentation profiling period (t49h-t55h) in TIM-2. A: A-OB1; B: A-OB2; C: A-PFP1; D: A-PFP2. 
Sugars arising from hemi(cellulose) such as xylose and glucose had a distinctive pattern of bacterial utilization when comparing A-OB1 to A-OB2. On A-OB1, utilization of glucose and xylose was low, since after 6 hours from fibre shot only $23.3 \%$ and $26.3 \%$ was used, respectively. On the other hand, A-OB2 fermentation presented a high utilization of xylose and glucose. After 6 hours from fibre shot, $84.6 \%$ of glucose and $85.9 \%$ of xylose disappeared from the system, indicating bacterial utilization. This difference might be explained by solubility. A-OB1 had a higher proportion of glucose and xylose present in the insoluble fraction than A-OB2 (data not shown). Degradation of insoluble fibres is challenging for gut microbes because it requires the adhesion of the bacteria to the cell wall polysaccharides which have lower accessible surface area and stronger hydrogen-bonding networks that holds the carbohydrates chain together $(50,51)$. Besides the difference found in solubility, A-OB2 had the capability to stimulate the growth of the bacterial group Ruminococcus, which was the main driver of clustering in PCoA (Figure 3). It was demonstrated that species from the genus Ruminoccocus, present in the human gut bacterial community, are able to degrade (hemi)cellulose (52).

Bacterial degradation of carbohydrates present in the two passion fruit peels tested was quite similar (Figure 6C-D). Pectic sugars from A-PFP1 and A-PFP2 were used in large proportions 6 hours after the fibre shot. For instance, 88.9\% and $89.1 \%$ of uronic acid from A-PFP1 and A-PFP2, respectively, was used in a period of 6 hours. Interestingly, glucose and xylose from hemi(cellulose) also disappeared from the system in high proportion $(88 \%$ and $92.5 \%$ on A-PFP1 and $85.1 \%$ and $87.1 \%$ on A-PFP2, respectively), indicating their utilization by resident bacteria. Like for A-OB2, Ruminococcus was the main responsible genus for the PCoA clustering of these samples (Figure 3) and increased after 73h fermentation period (Figure 4), and also here may explain the high proportion of glucose and xylose degradation from passion fruit peels.

\section{Conclusions}

This study revealed the potential prebiotic effect of fruit by-products in an in vitro model of the proximal colon.

With regard to orange bagasses, although the chemical composition was similar between the two tested samples, A-OB1and A-OB2 had a distinct fermentation profiling. A-OB2 was more efficient in stimulating a more diverse microbial community. The main genera stimulated by A-OB2 were Ruminococcus, Lachnospira, Roseburia, Dialister and Bacteroides. In turn, higher diversity after fermentation of A-OB2 was reflected in higher amounts of SCFA production when compared to A-OB1. Fermentation of A-OB2 generated similar amounts 
of total SCFA as inulin, our positive control. It also resulted in a smaller ratio of acetate/propionate than A-OB1, which has been shown to have hypolipidemic effects on the host. Additionally, analysis of degradation fate of carbohydrates showed that A-OB2 possessed a higher proportion of soluble than insoluble material, which might explain the differences found in fermentation profile between the two orange bagasses. Of note, this difference was revealed only with the analysis of degradation products during fermentation period.

Passion fruit peels fermentation resulted in smaller amounts of total SCFA when compared to control and inulin. Their fermentation resulted in almost negligible amounts of intermediate organic acids (lactate and succinate), which may be a sign of slow fermentation pace, which can be beneficial for colon health. Bacteroides, Lachnospira and Ruminococcus were the main bacterial genera stimulated by passion fruit peels.

This study provided evidence that isolated fibres from food by-products can be used as a tool for selective modulation of the gut microbiota. It also served to clarify carbohydrate utilization by the resident bacteria. Further experiments, either in vitro or in vivo, are needed to confirm our findings and to verify the effects of these fruit by-products on host health.

\section{Conflict of interest}

The authors declare that there are no conflicts of interest.

\section{Acknowledgments}

We thank Prof. Dr. Thiago Cardoso de Souza from Rio Grande do Norte Federal University - Brazil - for his assistance with R.

We also thank the companies Chá\&Cia, Phytomare and Orafti that kindly provided test compounds for the experiments. We kindly acknowledge Geraldo Bussolo and Zélia Maria Bussolo for collecting and sending test compound (orange bagasse) from Brazil.

The participation of volunteers that donated their faeces and made this study possible is greatly appreciated.

We thank Prof. Lubbert Dijkhuizen from Groningen University for critically reviewing our manuscript.

\section{Financial support}

Carlota Bussolo de Souza received a PhD scholarship from CNPq (National Council for Scientific and Technological Development) - Brazil - under the Program “Science without Borders" (246027/2012-6). 
The study was funded by the Centre for Healthy Eating \& Food Innovation (HEFI) of Maastricht University - campus Venlo. This research has been made possible with the support of the Dutch Province of Limburg. 


\section{References}

1. Helkar P, Sahoo A, Patil N. Review: Food industry by-products used as a functional food ingredients. Int J Waste Resour. 2016;6(3):1-6.

2. Parfitt J, Barthel M, Macnaughton S. Food waste within food supply chains: quantification and potential for change to 2050. Philos Trans R Soc London B Biol Sci. 2010;365(1554):3065-81.

3. Martins ZE, Pinho O, Ferreira I. Food industry by-products used as functional ingredients of bakery products. Trends Food Sci Technol. 2017;67:106-28.

4. Stephen AM, Champ MM-J, Cloran SJ, Fleith M, van Lieshout L, Mejborn H, et al. Dietary fibre in Europe: current state of knowledge on definitions, sources, recommendations, intakes and relationships to health. Nutr Res Rev. 2017 Jul 5;1-42.

5. Kovatcheva-Datchary P, Zoetendal EG, Venema K, de Vos WM, Smidt H. Tools for the tract: understanding the functionality of the gastrointestinal tract. Therap Adv Gastroenterol. 2009 Jul;2(4):9-22.

6. den Besten G, van Eunen K, Groen AK, Venema K, Reijngoud DJ, Bakker $\mathrm{BM}$. The role of short-chain fatty acids in the interplay between diet, gut microbiota, and host energy metabolism. J Lipid Res. 2013;54(9):232540.

7. Hamaker BR, Tuncil YE. A Perspective on the Complexity of Dietary Fiber Structures and Their Potential Effect on the Gut Microbiota. J Mol Biol. 2014 Nov 25;426(23):3838-50.

8. de Souza CB, Jonathan M, Saad SMI, Schols HA, Venema K. Characterization and in vitro digestibility of by-products from Brazilian food industry: cassava bagasse, orange bagasse and passion fruit peel. Bioact Carbohydrates Diet Fibre. 2018; (16):90-99.

9. Venema K, Nuenen M van, Smeets-Peeters M, Minekus M, Havenaar R. TNO's in vitro large intestinal model: an excellent screening tool for functional food and pharmaceutical research. Ernährung Nutr. 2000;24(12):559-64.

10. Rehman A, Heinsen F-A, Koenen ME, Venema K, Knecht H, Hellmig S, et al. Effects of probiotics and antibiotics on the intestinal homeostasis in a computer controlled model of the large intestine. BMC Microbiol. 2012;12(1):47.

11. Aguirre M, Eck A, Koenen ME, Savelkoul PHM, Budding AE, Venema K. Evaluation of an optimal preparation of human standardized fecal inocula for in vitro fermentation studies. J Microbiol Methods. 2015 Oct;117:78-84.

12. Aguirre M, Ramiro-Garcia J, Koenen ME, Venema K. To pool or not to pool? Impact of the use of individual and pooled fecal samples for in vitro fermentation studies. J Microbiol Methods. 2014;107C:1-7.

13. Maathuis AJ, van den Heuvel EG, Schoterman MH, Venema K. Galactooligosaccharides have prebiotic activity in a dynamic in vitro colon model using a (13)C-labeling technique. J Nutr. 2012;142(7):1205-12. 
14. Ramasamy US, Venema K, Gruppen H, Schols HA. The fate of chicory root pulp polysaccharides during fermentation in the TNO in vitro model of the colon (TIM-2). Bioact Carbohydrates Diet Fibre. 2014;4(1):48-57.

15. Ramasamy US, Gruppen H, Schols HA. Structural and water-holding characteristics of untreated and ensiled chicory root pulp. J Agric Food Chem. 2013 Jun;61(25):6077-85.

16. Thibault JF. Automated-method for the determination of pectic substances. Leb Technol. 1979;12(5):247-51.

17. Caporaso JG, Kuczynski J, Stombaugh J, Bittinger K, Bushman FD, Costello EK, et al. QIIME allows analysis of high-throughput community sequencing data. Vol. 7, Nature methods. United States; 2010. p. 335-6.

18. R Core Team. A language and environment for statistical computing. $\mathrm{R}$ Foundation for Statistical Computing. 2013.

19. Segata N, Izard J, Waldron L, Gevers D, Miropolsky L, Garrett WS, et al. Metagenomic biomarker discovery and explanation. Genome Biol. 2011 Jun;12(6):R60.

20. Langille MGI, Zaneveld J, Caporaso JG, McDonald D, Knights D, Reyes JA, et al. Predictive functional profiling of microbial communities using $16 \mathrm{~S}$ rRNA marker gene sequences. Nat Biotechnol. 2013 Sep;31(9):814-21.

21. Parks DH, Tyson GW, Hugenholtz P, Beiko RG. STAMP: statistical analysis of taxonomic and functional profiles. Bioinformatics. 2014 Nov;30(21):3123-4.

22. Russell WR, Hoyles L, Flint HJ, Dumas M-E. Colonic bacterial metabolites and human health. Curr Opin Microbiol. 2013 Jun;16(3):246-54.

23. Koh A, De Vadder F, Kovatcheva-Datchary P, Bäckhed F. From dietary fiber to host physiology: short-chain fatty acids as key bacterial metabolites. Cell. 2016 Jun 2;165(6):1332-45.

24. Flint HJ, Duncan SH, Louis P. 2 - Gut microbial ecology BT - Designing functional foods. In: Woodhead Publishing Series in Food Science, Technology and Nutrition. Woodhead Publishing; 2009. p. 38-67.

25. da Silva JK, Cazarin CBB, Bogusz Junior S, Augusto F, Maróstica Junior MR. Passion fruit (Passiflora edulis) peel increases colonic production of short-chain fatty acids in Wistar rats. LWT - Food Sci Technol. 2014;59(2, Part 2):1252-7.

26. Bolca S, Van de Wiele T, Possemiers S. Gut metabotypes govern health effects of dietary polyphenols. Curr Opin Biotechnol. 2013 Apr;24(2):220-5.

27. Delzenne NM, Williams CM. Prebiotics and lipid metabolism. Curr Opin Lipidol. 2002 Feb;13(1):61-7.

28. Demigne C, Morand C, Levrat MA, Besson C, Moundras C, Remesy C. Effect of propionate on fatty acid and cholesterol synthesis and on acetate metabolism in isolated rat hepatocytes. Br J Nutr. 1995 Aug;74(2):209-19.

29. Lopez HW, Levrat-Verny MA, Coudray C, Besson C, Krespine V, Messager A, et al. Class 2 resistant starches lower plasma and liver lipids and 
improve mineral retention in rats. J Nutr. 2001 Apr;131(4):1283-9.

30. Delzenne NM, Cani PD. Interaction between obesity and the gut microbiota: relevance in nutrition. Annu Rev Nutr. 2011;31:15-31.

31. Koenen ME, Cruz Rubio JM, Mueller M, Venema K. The effect of agave fructan products on the activity and composition of the microbiota determined in a dynamic in vitro model of the human proximal large intestine. J Funct Foods. 2016;22:201-10.

32. Macfarlane GT, Macfarlane S. Bacteria, colonic fermentation, and gastrointestinal health. J AOAC Int. 2012;95(1):50-60.

33. Ou J, DeLany JP, Zhang M, Sharma S, O’Keefe SJD. Association between low colonic short-chain fatty acids and high bile acids in high colon cancer risk populations. Nutr Cancer. 2012;64(1):34-40.

34. Khodaei N, Fernandez B, Fliss I, Karboune S. Digestibility and prebiotic properties of potato rhamnogalacturonan I polysaccharide and its galactose-rich oligosaccharides/oligomers. Carbohydr Polym. 2016;136:1074-84.

35. Geypens B, Claus D, Evenepoel P, Hiele M, Maes B, Peeters M, et al. Influence of dietary protein supplements on the formation of bacterial metabolites in the colon. Gut. 1997 Jul;41(1):70-6.

36. Aguirre M, Jonkers DMAE, Troost FJ, Roeselers G, Venema K. In vitro characterization of the impact of different substrates on metabolite production, energy extraction and composition of gut microbiota from lean and obese subjects. PLoS One. 2014;9(11):e113864.

37. Sáyago-Ayerdi SG, Zamora-Gasga VM, Venema K. Prebiotic effect of predigested mango peel on gut microbiota assessed in a dynamic in vitro model of the human colon (TIM-2). Food Res Int. 2017;

38. Le Chatelier E, Nielsen T, Qin J, Prifti E, Hildebrand F, Falony G, et al. Richness of human gut microbiome correlates with metabolic markers. Nature. 2013;500(7464):541-6.

39. Cotillard A, Kennedy SP, Kong LC, Prifti E, Pons N, Le Chatelier E, et al. Dietary intervention impact on gut microbial gene richness. Nature. 2013;500(7464):585-8.

40. Le Chatelier E, Nielsen T, Qin J, Prifti E, Hildebrand F, Falony G, et al. Richness of human gut microbiome correlates with metabolic markers. Nature. 2013;500(7464):541-6.

41. Rajilić-Stojanović M, de Vos WM. The first 1000 cultured species of the human gastrointestinal microbiota. FEMS Microbiol Rev. 2014;38(5):996-1047.

42. Flint HJ, Scott KP, Duncan SH, Louis P, Forano E. Microbial degradation of complex carbohydrates in the gut. Vol. 3, Gut Microbes. 2012. p. 289306.

43. Macfarlane S, Macfarlane GT, Cummings JH. Review article: Prebiotics in the gastrointestinal tract. Aliment Pharmacol Ther. 2006;24(5):701-14.

44. Martens EC, Koropatkin NM, Smith TJ, Gordon JI. Complex glycan catabolism by the human gut microbiota: the Bacteroidetes Sus-like 
paradigm. J Biol Chem. 2009 Sep;284(37):24673-7.

45. Martens EC, Kelly AG, Tauzin AS, Brumer H. The devil lies in the details: How variations in polysaccharide fine-structure impact the physiology and evolution of gut microbes. J Mol Biol. 2014;426(23):3851-65.

46. Aguirre M, Eck A, Koenen ME, Savelkoul PHM, Budding AE, Venema K. Diet drives quick changes in the metabolic activity and composition of human gut microbiota in a validated in vitro gut model. Res Microbiol. 2016;167(2):114-25.

47. Vidal-Lletjos S, Beaumont M, Tome D, Benamouzig R, Blachier F, Lan A. dietary protein and amino acid supplementation in inflammatory bowel disease course: what impact on the colonic mucosa? Nutrients. 2017 Mar;9(3).

48. De Filippo C, Cavalieri D, Di Paola M, Ramazzotti M, Poullet JB, Massart S, et al. Impact of diet in shaping gut microbiota revealed by a comparative study in children from Europe and rural Africa. Proc Natl Acad Sci U S A. 2010 Aug;107(33):14691-6.

49. Flint HJ, Duncan SH, Louis P. The impact of nutrition on intestinal bacterial communities. Curr Opin Microbiol. 2017 Aug;38:59-65.

50. Cockburn DW, Koropatkin NM. Polysaccharide Degradation by the intestinal microbiota and its influence on human health and disease. J Mol Biol. 2016 Aug 14;428(16):3230-52.

51. Lynd LR, Weimer PJ, van Zyl WH, Pretorius IS. Microbial cellulose utilization: fundamentals and biotechnology. Microbiol Mol Biol Rev. 2002 Sep;66(3):506-77.

52. Chassard C, Delmas E, Robert C, Lawson PA, Bernalier-Donadille A. Ruminococcus champanellensis sp. nov., a cellulose-degrading bacterium from human gut microbiota. Int J Syst Evol Microbiol. 2012 Jan;62(Pt 1):138-43. 


\section{Supplemental material}

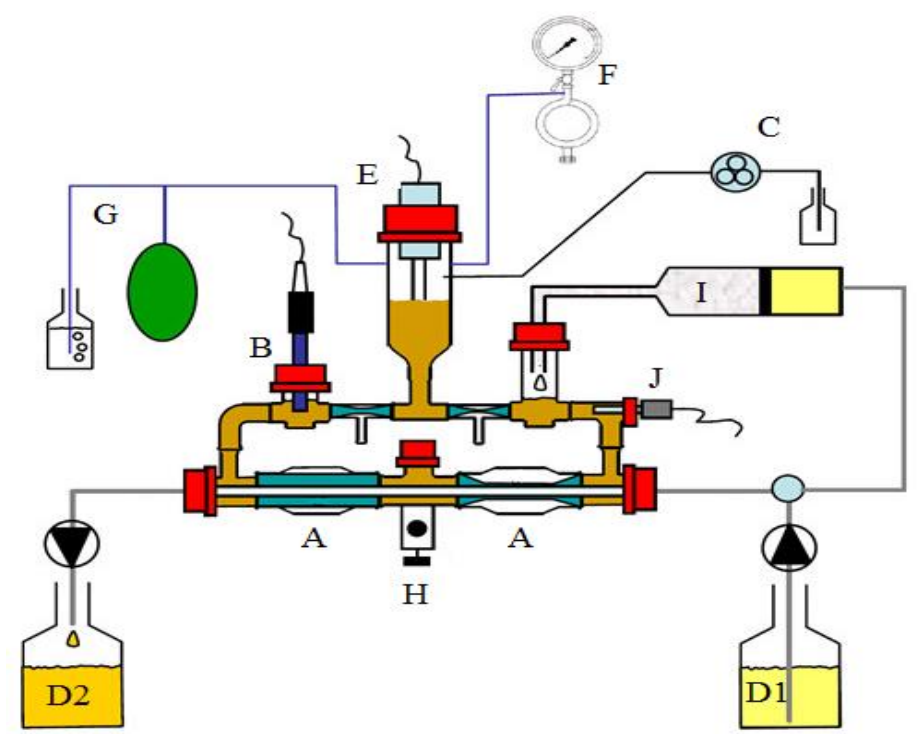

Supplemental Figure 1: TIM-2 system. A) peristaltic compartments with a dialysis membrane inside that removes microbial metabolites and supplies the system with liquid dialysate, B) $\mathrm{pH}$ sensor, $\mathrm{C}$ ) $\mathrm{NaOH}$ secretion for $\mathrm{pH}$ maintenance, D) dialysate system (D1-dialysate in, D2-dialysate out), E) level sensor, F) gaseous $\mathrm{N}_{2}$ inlet to keep the system anaerobic, G) gas outlet, H) sampling port, I) feeding syringe with test compound, J) temperature sensor to maintain the system at body temperature $\left(37^{\circ} \mathrm{C}\right)$. Adapted from (10).

A

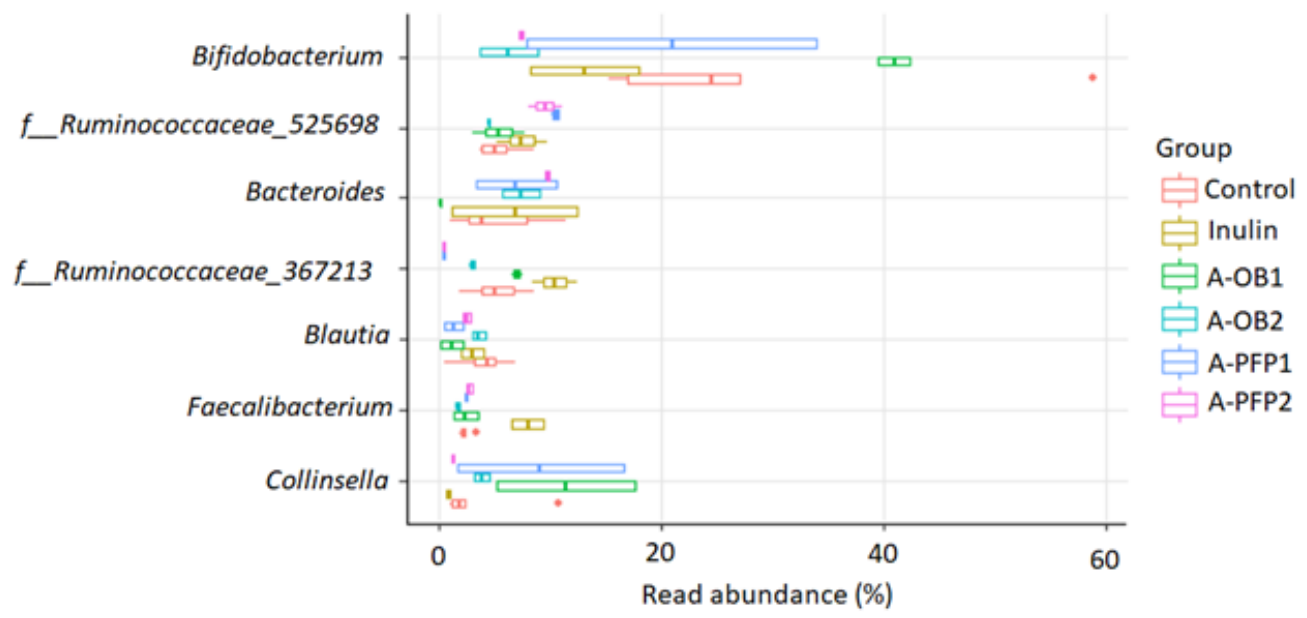




\section{B}

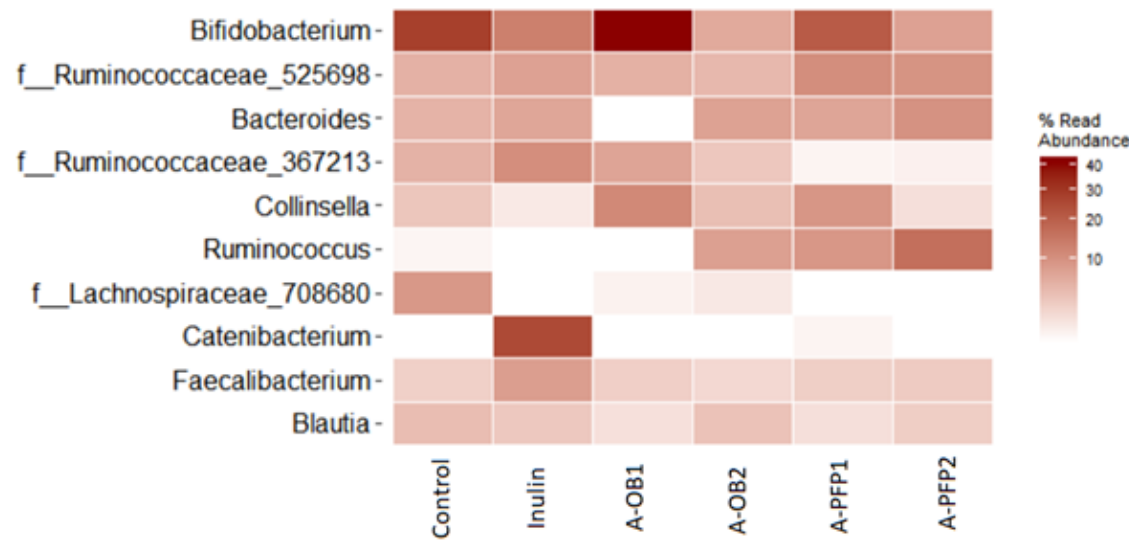

Supplemental Figure 2: Boxplot (A) and corresponding heatmap (B) for the top 10 OTUs driving the differences found in samples.

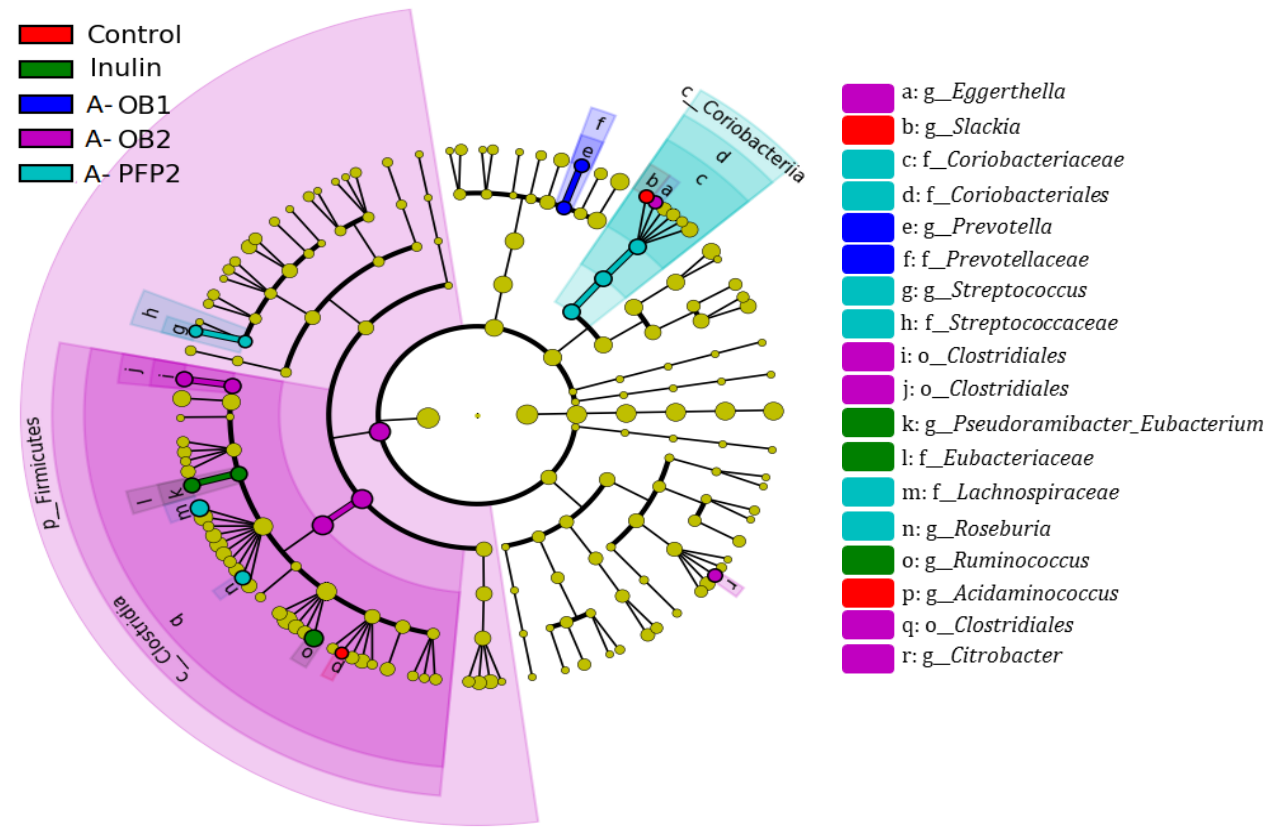



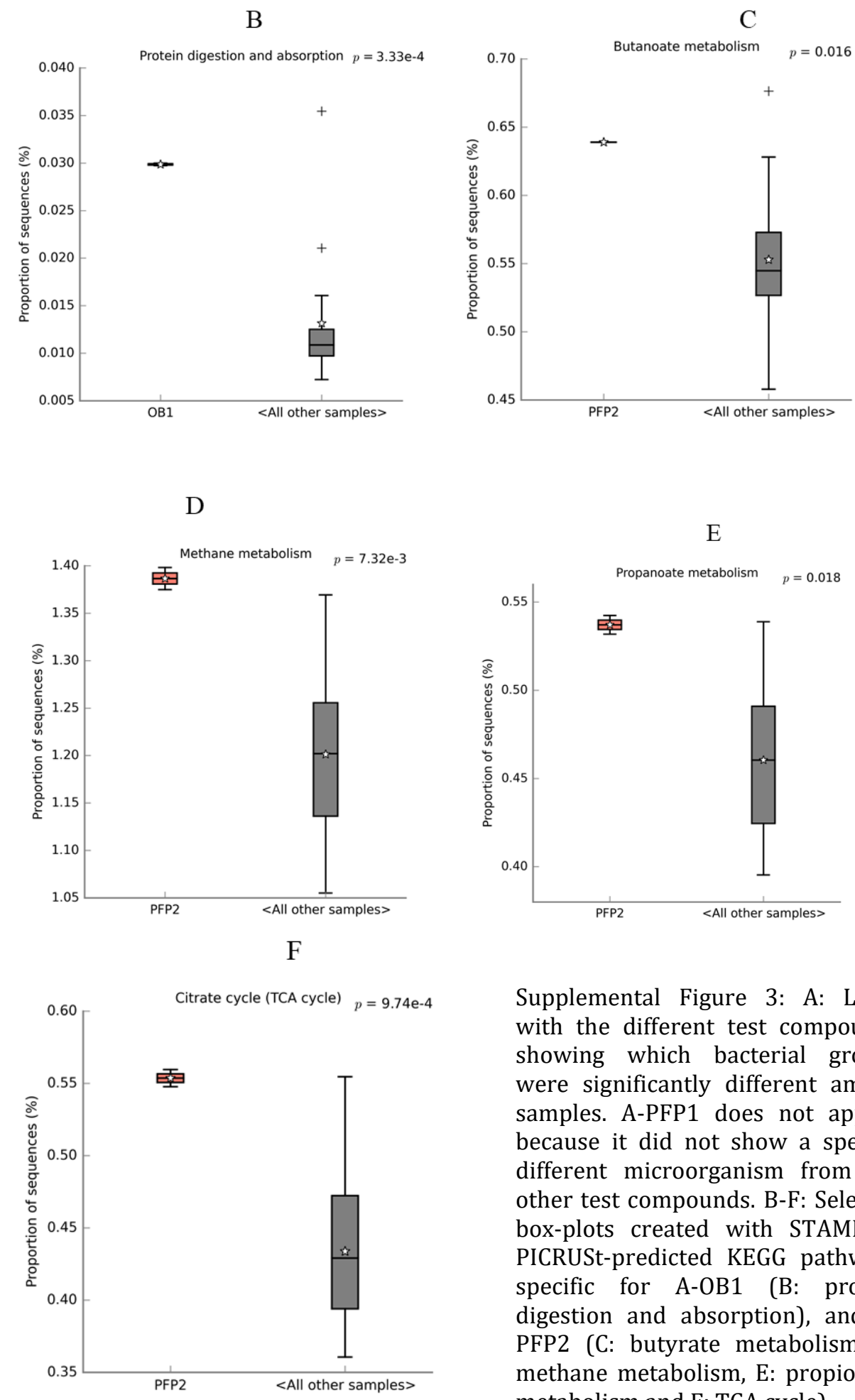

Supplemental Figure 3: A: LEfSe with the different test compounds showing which bacterial groups were significantly different among samples. A-PFP1 does not appear because it did not show a specific different microorganism from the other test compounds. B-F: Selected box-plots created with STAMP of PICRUSt-predicted KEGG pathways specific for A-OB1 (B: protein digestion and absorption), and APFP2 (C: butyrate metabolism, D: methane metabolism, E: propionate metabolism and F: TCA cycle). 
A

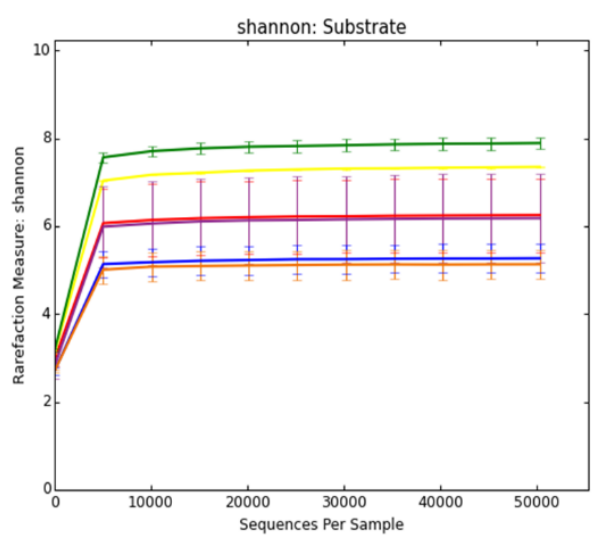

B

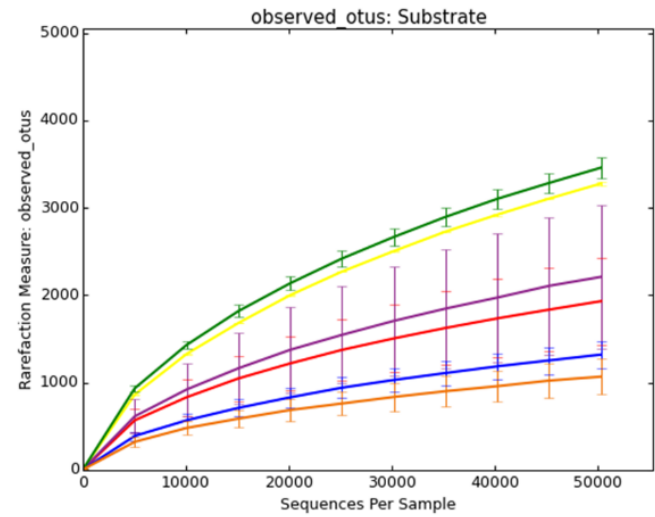

Control

Inulin

A-OB1

a-OB2

A-PFP1

A-PFP2
Supplemental Figure 4: A: Shannon index at t73h with test compounds. Fermentation of A-OB2 resulted in the highest diversity in bacterial population, whereas A-OB1 showed the least diverse composition. B: Observed OTUs at time $73 \mathrm{hs}$ with test compounds. A-OB2 had the highest number of observed OTUs. 
Supplemental Table 1: Predicted KEGG pathways based on observed OTUs in the different TIM-2 samples from fermentation experiments with AIS fractions of the different food-by-products.

\begin{tabular}{|c|c|c|c|c|}
\hline Predicted KEGG pathways & $\begin{array}{l}\text { mean } \\
\text { rel. freq, } \\
(\%)\end{array}$ & $\begin{array}{l}\text { mean rel. } \\
\text { freq, }(\%)\end{array}$ & p-values & $\begin{array}{c}\text { p-values } \\
\text { (Bonferroni } \\
\text { corrected) }\end{array}$ \\
\hline \multicolumn{5}{|c|}{ A-0B1 vs. the other three substrates (A-OB2, A-PFP1 and A-PFP2) } \\
\hline & A-0B1 & $\begin{array}{l}\text { All other } \\
\text { samples }\end{array}$ & & \\
\hline African trypanosomiasis & 0.024 & 0.009 & $2.59 \mathrm{E}-05$ & 0.008 \\
\hline $\begin{array}{l}\text { Cell cycle - Caulobacter } \\
\text { Chagas disease (American }\end{array}$ & 0.536 & 0.504 & $2.85 \mathrm{E}-05$ & 0.009 \\
\hline trypanosomiasis) & 0.022 & 0.008 & $3.52 \mathrm{E}-05$ & 0.012 \\
\hline D-Alanine metabolism & 0.121 & 0.106 & 7.17E-05 & 0.024 \\
\hline $\begin{array}{l}\text { Function unknown } \\
\text { Isoquinoline alkaloid }\end{array}$ & 1.228 & 1.164 & $5.92 \mathrm{E}-06$ & 0.002 \\
\hline $\begin{array}{l}\text { biosynthesis } \\
\text { Nicotinate and nicotinamide }\end{array}$ & 0.058 & 0.045 & $2.53 \mathrm{E}-06$ & $8.29 \mathrm{E}-04$ \\
\hline metabolism & 0.542 & 0.464 & $2.78 \mathrm{E}-05$ & 0.009 \\
\hline Nucleotide excision repair & 0.507 & 0.428 & $1.15 \mathrm{E}-05$ & 0.004 \\
\hline One carbon pool by folate & 0.667 & 0.637 & $7.30 \mathrm{E}-05$ & 0.024 \\
\hline Peptidoglycan biosynthesis & 0.931 & 0.859 & $2.83 \mathrm{E}-06$ & 0.001 \\
\hline $\begin{array}{l}\text { Prenyltransferases } \\
\text { Protein digestion and }\end{array}$ & 0.351 & 0.308 & $2.43 \mathrm{E}-06$ & 0.001 \\
\hline absorption & 0.030 & 0.013 & $1.02 \mathrm{E}-06$ & $3.33 \mathrm{E}-04$ \\
\hline Pyrimidine metabolism & 2.050 & 1.870 & $3.17 \mathrm{E}-07$ & $1.04 \mathrm{E}-04$ \\
\hline $\begin{array}{l}\text { Ribosome } \\
\text { Ribosome biogenesis in }\end{array}$ & 2.799 & 2.454 & $2.42 \mathrm{E}-06$ & $7.95 \mathrm{E}-04$ \\
\hline eukaryotes & 0.077 & 0.057 & $2.39 \mathrm{E}-05$ & 0.008 \\
\hline RNA polymerase & 0.199 & 0.171 & $3.42 \mathrm{E}-05$ & 0.011 \\
\hline $\begin{array}{l}\text { Transcription factors } \\
\text { Ubiquinone and other }\end{array}$ & 1.774 & 1.863 & $2.41 \mathrm{E}-05$ & 0.008 \\
\hline terpenoid-quinone biosynthesis & 0.139 & 0.082 & $1.15 \mathrm{E}-07$ & $3.77 \mathrm{E}-05$ \\
\hline Ubiquitin system & 0.023 & 0.009 & $4.21 \mathrm{E}-05$ & 0.014 \\
\hline \multicolumn{5}{|c|}{ A-OB2 vs. the other three substrates (A-OB1, A-PFP1 and A-PFP2) } \\
\hline & A-OB2 & $\begin{array}{l}\text { All other } \\
\text { samples }\end{array}$ & & \\
\hline Lysine biosynthesis & 0.882 & 0.937 & $7.19 \mathrm{E}-06$ & 0,002 \\
\hline
\end{tabular}




\section{A-PFP1 vs. the other three substrates (A-OB1, A-OB2 and A-PFP2)}

$$
\begin{array}{ll}
\text { A-PFP1 } & \begin{array}{l}
\text { All other } \\
\text { samples }
\end{array}
\end{array}
$$

Biosynthesis and

biodegradation of secondary

metabolites

$\begin{array}{llll}0.067 & 0.053 & 3.59 \mathrm{E}-05 & 0.012 \\ 0.001 & 0.003 & 5.77 \mathrm{E}-05 & 0.019 \\ 0.148 & 0.203 & 9.55 \mathrm{E}-05 & 0.031\end{array}$

Carotenoid biosynthesis

0.148

0.203

9.55E-05

0.031

\begin{tabular}{|c|c|c|c|c|}
\hline & A-PFP2 & $\begin{array}{l}\text { All other } \\
\text { samples }\end{array}$ & & \\
\hline \multirow{2}{*}{$\begin{array}{l}\text { Butanoate metabolism } \\
\text { Carbon fixation pathways in } \\
\text { prokaryotes }\end{array}$} & 0.639 & 0.553 & $4.86 \mathrm{E}-05$ & 0.016 \\
\hline & 0.988 & 0.829 & $9.21 \mathrm{E}-07$ & $3.02 \mathrm{E}-04$ \\
\hline Citrate cycle (TCA cycle) & 0.554 & 0.434 & 2.97E-06 & $9.74 \mathrm{E}-04$ \\
\hline Energy metabolism & 0.938 & 0.792 & $5.41 \mathrm{E}-06$ & 0.002 \\
\hline Ethylbenzene degradation & 0.050 & 0.032 & $1.03 \mathrm{E}-05$ & 0.003 \\
\hline Fatty acid biosynthesis & 0.518 & 0.411 & $5.89 \mathrm{E}-06$ & 0.002 \\
\hline Insulin signalling pathway & 0.112 & 0.095 & 0.000148 & 0.048 \\
\hline Linoleic acid metabolism & 0.083 & 0.059 & $5.89 \mathrm{E}-06$ & 0.002 \\
\hline Lipid biosynthesis proteins & 0.621 & 0.551 & $3.54 \mathrm{E}-06$ & $1.16 \mathrm{E}-03$ \\
\hline Methane metabolism & 1.387 & 1.201 & $2.23 \mathrm{E}-05$ & 0.007 \\
\hline Mismatch repair & 0.854 & 0.804 & $1.14 \mathrm{E}-05$ & 0.004 \\
\hline Pathways in cancer & 0.054 & 0.040 & $1.59 \mathrm{E}-05$ & 0.005 \\
\hline Phenylalanine metabolism & 0.179 & 0.150 & $5.74 \mathrm{E}-06$ & 0.002 \\
\hline Phenylpropanoid biosynthesis & 0.148 & 0.203 & $8.58 \mathrm{E}-05$ & 0.028 \\
\hline Primary bile acid biosynthesis & 0.028 & 0.044 & $4.00 \mathrm{E}-07$ & 0.000 \\
\hline Propanoate metabolism & 0.537 & 0.460 & $5.40 \mathrm{E}-05$ & 0.018 \\
\hline $\begin{array}{l}\text { Protein export } \\
\text { Proximal tubule bicarbonate }\end{array}$ & 0.629 & 0.594 & $7.54 \mathrm{E}-05$ & 0.025 \\
\hline reclamation & 0.022 & 0.008 & $3.81 \mathrm{E}-07$ & $1.25 \mathrm{E}-04$ \\
\hline Pyruvate metabolism & 1.122 & 0.992 & $1.63 \mathrm{E}-06$ & $5.35 \mathrm{E}-04$ \\
\hline $\begin{array}{l}\text { Renal cell carcinoma } \\
\text { RIG-I-like receptor signalling }\end{array}$ & 0.010 & 0.002 & 0.000104 & 0.034 \\
\hline $\begin{array}{l}\text { pathway } \\
\text { Secondary bile acid }\end{array}$ & 0.004 & 0.002 & 0.000101 & 0.033 \\
\hline biosynthesis & 0.028 & 0.044 & $3.90 \mathrm{E}-07$ & $1.28 \mathrm{E}-04$ \\
\hline Starch and sucrose metabolism & 1.106 & 1.263 & $1.95 \mathrm{E}-06$ & $6.38 \mathrm{E}-04$ \\
\hline Tetracycline biosynthesis & 0.167 & 0.109 & $2.12 \mathrm{E}-05$ & 0.007 \\
\hline Transcription machinery & 1.099 & 0.939 & $2.54 \mathrm{E}-05$ & 0.008 \\
\hline
\end{tabular}

\section{A-PFP2 vs. the other three substrates (A-OB1, A-OB2 and A-PFP1)}




\section{Chapter 6}

The gut microbiota from lean and obese subjects contribute differently to the fermentation of arabinogalactan and inulin

Published as:

Aguirre M, De Souza CB, Venema K. The gut microbiota from lean and obese subjects contribute differently to the fermentation of arabinogalactan and inulin. PLoS One. 2016 Jul 1;11 (7). 


\begin{abstract}
An aberrant metabolic activity or a compositional alteration of the gut microbiota has been proposed as a factor that makes us more prone to disease. Therefore, we explored the effect of two dietary fibres (arabinogalactan and inulin) on the microbiota from lean and obese subjects during $72 \mathrm{~h}$ in vitro fermentation experiments using the validated TNO dynamic in vitro model of the proximal colon: TIM-2. Metabolically, arabinogalactan fermentation showed a higher production of propionate $(45.2 \mathrm{mmol})$ when compared to $n$-butyrate (19.1 mmol) in the obese microbiota fermentations. In general, lean microbiota produced more $n$-butyrate from the fermentation of both substrates when compared to the obese microbiota. Furthermore, the obese microbiota extracted more energy from the fermentation of both fibres $-46.9 \mathrm{kcal}$ by the obese microbiota versus $44.6 \mathrm{kcal}$ by the lean microbiota when using inulin, and 40.5 kcal by the obese microbiota versus $37.3 \mathrm{kcal}$ by the lean microbiota when using arabinogalactan as substrate. Compositionally, bacteria belonging to Gemmiger, Dorea, Roseburia, Alistipes, Lactobacillus and Bifidobacterium genera were found to be highly abundant or stimulated by the prebiotics in the lean microbiota suggesting a potential role in leanness. Furthermore, a significant correlation between known butyrogenic strains including $B$. adolescentis, an unclassified Bifidobacterium and F. prausnitzii with this metabolite in the fermentation of inulin in both microbiotas was found. Although supplementary in vivo studies are needed, the current study provides more evidence for the consumption of specific ingredients with the aim of modulating the gut microbiota in the context of obesity.
\end{abstract}




\section{Introduction}

The discovery of the potential impact of the gut microbiota on human health and disease has fuelled research on characterizing the role that this community plays in the causality or prevention of many diseases elicited by dangerous lifestyles such as sedentary and bad eating habits, among others $(1,2)$.

Part of the efforts have been focused on identifying a balanced and thus, healthy community (3). Though provocative, it is difficult to define a "most desirable" composition for the human gut microbiota. Reports providing contradictory findings, due to either $i$ ) a large inter-individual variation or ii) the application of different analytic methods, are at the order of the day. However, another factor that seems to play an important role in influencing health and disease, besides the community composition, is the interaction of the microbial metabolites with the host. The fermentation of dietary fibre by the gut microbiota leads primarily to the production of short-chain fatty acids (SCFA; mainly acetate, propionate and butyrate) and the gases hydrogen, methane and carbon dioxide (4). Furthermore, branched-chain fatty acids (BCFA; mainly iso-butyrate and isovalerate often accompanied by phenol and ammonia production) are also produced to a lesser extent but these mostly originate from protein fermentation (5). A proposed mechanism by which fibre may protect us against obesity is based on the beneficial effects that such metabolites have on host energy balance, e.g. by mediating the secretion of gut hormones involved in the regulation of energy metabolism and food intake (including leptin, peptide YY and glucagonlike peptide-1) $(6,7)$. Thus, it may be tempting to say that high intake of fibre would be a way to reduce the risk of obesity $(8,9)$. After all, it is estimated that the production of SCFA by the microbiota accounts for 5 to $10 \%$ of total dietary energy requirements in humans (10). However, recent research has questioned such risk-reduction role. There is growing evidence indicating that the production of SCFA differs between the microbiota originating from obese and lean individuals (hereafter referred to as obese and lean microbiota). Such difference lies in the fact that the obese microbiota may produce more SCFA which could be translated into more energy extraction from diet $(11,12)$. As a consequence, more energy extracted from diet may be stored as fat, promoting weight gain of the host. Such mechanisms place fibre fermentation by the gut microbiota as a causative factor in obesity. Still, as previously remarked, there is a lack of consistency, different studies show contrasting results by finding either no correlation between fibre and weight gain/obesity, a reverse trend or effects to be substrate dependent (12-14). Vast amounts of research are needed to answer the chicken or the egg causality dilemma before any strategy can be designed with the aim of manipulating the gut microbiota in the context of 
obesity. Currently, there are a limited number of in vitro fermentation experiments mimicking the fermentation of different substrates by human obese or lean microbiota. So far, these studies have provided evidence about the metabolic adaptation of the microbiota in relation to different nutrient loads or single testing of specific prebiotics, as well as the plasticity of the microbiota in configuring the structure of the community in response to these kind of interventions (13,15-19). Importantly, these studies have also endorsed in vitro systems as tools facilitating the medium to high-throughput validation of multiple hypotheses at lower costs with no ethical constraints when compared to human or animal studies.

The purpose of the current study was to compare the profiles of fermentation of arabinogalactan (AG) and the well-studied prebiotic inulin (IN) by obese or lean microbiota. Both AG and IN are natural polysaccharides commonly found in foods. They have been found to be fermented by human intestinal bacteria and stimulate the production of SCFA and the growth of specific bacteria generally believed to be beneficial to the host (20-23). AG is an interesting compound to evaluate not only because its potential to improve gut barrier function (22) but also because it has been observed that it may induce production of (both pro and anti- inflammatory) cytokines $(24,25)$, factors which both may play an important role in inflammation. In the context of obesity, low grade inflammation has been suggested to contribute to the development of insulin and leptin resistance (26). In order to compare the two compounds, we performed $72 \mathrm{~h}$ fermentation experiments in the validated TNO dynamic in vitro model of the proximal colon (TIM-2), which was inoculated with either obese or lean microbiota. The present work brings evidence about how fermentable carbohydrates are differently used by the microbiota from lean and obese subjects which contributes to the understanding on how dietary compounds could be used as therapeutic tools in obesity.

\section{Materials and Methods}

\section{Gut microbiota}

The inocula used for the TIM-2 experiments consisted of an active, pooled faecal microbiota prepared from: $i$ ) 8 healthy lean volunteers (male: $\mathrm{n}=4$, female: $\mathrm{n}=$ 4 , average age $=31$ years (range: $25-42$ ), BMI $=20 \pm 1.5 \mathrm{~kg} / \mathrm{m}^{2}$ ); ii) 7 healthy obese volunteers (male: $\mathrm{n}=3$, female: $\mathrm{n}=4$, average age $=51$ years (range: 2968 ), $\mathrm{BMI}=32 \pm 1.2 \mathrm{~kg} / \mathrm{m}^{2}$. We have previously shown that pooling does not result in an aberrant microbiota composition or activity (27). The exclusion criteria for lean and obese volunteers included the use of antibiotics during the preceding 3 
months, gastrointestinal disease, severe chronic disease or food allergy and intake of probiotics and prebiotics.

Whole faecal samples were self-collected in a container kit that was maintained under anaerobiosis by using anaerobic packs (AnaeroGen ${ }^{\mathrm{TM}}$, Oxoid, Cambridge, UK). A sample aliquot (100 $\mathrm{mg}$ ) from each individual donation was collected in an anaerobic cabinet $\left(80 \% \mathrm{~N}_{2}, 10 \% \mathrm{CO}_{2}, 10 \% \mathrm{H}_{2}\right)$, snap-frozen in liquid nitrogen $\left(-196^{\circ} \mathrm{C}\right)$ and stored at $-80^{\circ} \mathrm{C}$ for measurement of metabolites (SCFA and BCFA). Faeces were homogenized under anaerobic conditions as described by Aguirre et al. (28). The resulting culture homogenate was aliquoted and snap-frozen in liquid nitrogen. This microbiota was stored at $-80^{\circ} \mathrm{C}$ before inoculation in TIM2.

\section{Gut fermentation experiments}

The TIM-2 system was flushed with $\mathrm{N}_{2}$ prior to the introduction of the inoculum for $3 \mathrm{~h}$ and it was maintained under this condition at $37^{\circ} \mathrm{C}$ for $96 \mathrm{~h}$ with the $\mathrm{pH}$ kept at or above 5.8 by automatic titration with $2 \mathrm{M} \mathrm{NaOH}$. A $30 \mathrm{ml}$ portion of culture homogenate was used to inoculate the units for each experiment. The microbiota was left to adapt ( $16 \mathrm{~h}$ ) to the new environment after inoculation and during this period the basal simulated ileal efflux medium (SIEM) was gradually introduced into the system in a total volume of $40 \mathrm{ml}$. After the adaptation, the culture was deprived from any medium for $2 \mathrm{~h}$ (starvation). A volume of $180 \mathrm{ml}$ of the different diets and control was administrated over the $72 \mathrm{~h}$ of the test period at a rate of $2.5 \mathrm{ml} / \mathrm{h}$.

In order to remove water and fermentation products from the lumen, a dialysate system (described in detail by van Nuenen et al. (29)), consisting of a semipermeable hollow membrane, ran through the lumen. For all the experiments, the speed of the dialysis fluid was set at $1.5 \mathrm{ml} / \mathrm{min}$.

After 24 and $48 \mathrm{~h}$ of fermentation $25 \mathrm{ml}$ of lumen sample was removed from the system to mimic the transit of material from the proximal and reaching the distal colon (30). Luminal and dialysate samples were taken after $t=0,24,48$ and 72 h. In all cases samples were snap-frozen in liquid nitrogen and stored $\left(-80^{\circ} \mathrm{C}\right)$ until analysis.

\section{Fermentation media}

During the adaptation period (16 h) all TIM-2 units were fed with SIEM as described by Maathuis et al. (31). After the $2 \mathrm{~h}$ starvation period, the units were fed with preparations which were made containing approximately $7.5 \mathrm{~g}$ of AG or IN instead of the standard carbohydrates in SIEM. The specific AG used in this study was (+)-Arabinogalactan- from larch wood (Sigma-Aldrich, St Louis, USA) 
with a molecular weight ranging from $72-92 \mathrm{kDa}$ and $\geq 84.8 \%$ purity. The IN tested had an average degree of polymerization (DP) of 9; 84.9\% > DP5 (Sensus, Frutafit ${ }^{\circledR}$ IQ, Roosendaal, the Netherlands). Control experiments were performed in parallel to the experiments testing either AG or IN. SIEM was used to feed the microbiota in such controls.

\section{Analysis of SCFA (acetate, propionate, and $n$-butyrate) and BCFA (iso-butyrate and iso-valerate)}

Samples were prepared and analyzed as described previously (13). Before centrifuging, the faecal aliquots from the individuals were suspended in PBS $11: 1$; w:w). Briefly, both suspended aliquots and TIM-2 luminal samples were centrifuged $\left(12.000 \mathrm{rpm}\right.$ at $4{ }^{\circ} \mathrm{C}$ for $\left.10 \mathrm{~min}\right)$. To the clear supernatant a mixture of formic acid (20\%), methanol and 2-ethyl butyric acid (internal standard, 2 $\mathrm{mg} / \mathrm{ml}$ in methanol) was added. A $3 \mu \mathrm{l}$ sample with a split ratio of 75.0 was injected on a GC-column (ZB-5HT inferno, ID $0.52 \mathrm{~mm}$, film thickness $0.10 \mathrm{um}$; Zebron; phenomenex, USA) in a Shimadzu GC-2014 gas chromatograph. Standard curves were obtained by injecting calibrated quantities of a blend of volatile fatty acids and amounts were calculated from the graph obtained correlating peak height and time measured (all reagents from Sigma-Aldrich with the exception of formic acid which was from Merck).

\section{Energy extraction}

Energy extraction in the form of SCFA was calculated using the following values of kJ mol-1 for acetate, propionate and $n$-butyrate respectively: 874, 1536 and $2192(32,33)$.

\section{Characterization of bacterial populations}

RNA was isolated from luminal samples using standard molecular biology kits from ZYMO Research (Zymo Research Co., CA, USA) following manufacturer's instructions. Reverse-transcriptase amplification of the 16S rRNA gene (V3-V4), barcoding and library preparation (1st step PCR and 2nd PCR) were performed by BaseClear, Leiden, the Netherlands.

Short paired-end sequence reads were generated using the Illumina MiSeq system and converted into FASTQ files using the BCL2FASTQ pipeline version 1.8.3. Quality trimming was applied based on Phred quality scores. Subsequently, the Illumina paired reads were merged into single reads (so-called pseudoreads) through sequence overlap (16S rRNA V3-V4 region of about 500bp). Chimeric pseudoreads were removed and the remaining reads were aligned to a combination of the GreenGenes and RDP 16 S gene databases $(34,35)$. Based on 
the alignment scores of the pseudoreads, the taxonomic classes were assigned by associating each pseudoread to the best matching Operational Taxonomic Unit (OTU). The taxonomic depth of the lineage was based on the identity threshold of the rank; Species 99\%, Genus 97\%, Family 95\%, Order 90\%, Class 85\%, Phylum $80 \%$.

\section{Data analysis}

The experiments were performed in series of two per tested substrate $(n=2)$. These replicates were conducted for each microbiota (i.e. lean or obese). To avoid unnecessary repetition, this is not indicated further in the text or graphs in the results section. Results are displayed as average of these duplicates. For simplicity of reading, substrates in the following sections are tagged with the letter L or O (e.g. substrate-L, substrate-O) in order to refer to the fermentation experiments using the inoculum from lean (-L) or obese (-O) subjects.

Statistical analyses for determining the differences in metabolite production and energy extraction in the faecal samples from each individual were performed (SPSS for Windows, version 21, SPSS, Chicago, US). Comparison between the two groups (lean and obese) was performed using t-test with significance $\mathrm{p}<0.05$. For the calculation of fold compositional changes, the ratio between a sampling time point and $\mathrm{t} 0$ was calculated (i.e., $\mathrm{t} 72 / \mathrm{t} 0$ ). Then the ratio for this value and the control was then determined to obtain fold changes compared to control. A value equal to 1 indicates no change; a value of $>1$ indicates an increase; and a value of $<1$ indicates a decrease of the respective microbial genera.

A correlation analysis was performed in order to test if the metabolites measured would positively or negatively correlate with the different bacterial groups fed with either AG or IN. To these means, differentially abundant bacterial species growing on the different tested substrates were calculated from a ratio based from the specific growth of the species found on each substrate and the control. Spearman correlations were calculated between the ratio of the species identified as being differentially abundant and the measured amounts of metabolites produced (SPSS for Windows, version 21, SPSS, Chicago, US). Correlations were considered significant at the 0.01 level (2-tailed).

\section{Ethics Statement}

Studies using faecal donations from healthy volunteers do not require medical ethical committee approval in the Netherlands since they are considered as noninvasive. However, volunteers who donated the inoculum were informed prior to initiating the study and their participation was considered after providing a signed informed consent. The group of obese donors were recruited at 
Maastricht University Medical Center (the Netherlands). These were patients from the university medical center who voluntarily responded to a recruiting call inviting to donate their faeces. The group of lean donors were recruited at TNO (the Netherlands). These participants responded to an advertisement inviting subjects to voluntarily collect their faeces. Bouke Salden and Carlota Bussolo de Souza personally collected the faecal samples from the participants who exclusively donated their faeces for the present study. Bouke Salden received the faecal samples directly from the obese participants and none of the authors was involved in the direct collection of these samples. Carlota Bussolo de Souza received the faecal samples directly from the lean participants. The origins of the both lean and obese faecal donations were blinded using a code whose identity was known only by the responsible scientist (Carlota Bussolo de Souza, coauthor of the present study). Results in this manuscript are referred to an individual sample or a pooled faecal inoculum and do not directly refer to a particular person.

\section{Results}

\section{Screening of metabolites and energy extraction in faeces from the volunteers}

The average amount of SCFA found in the faeces from lean and obese subjects were not statistically different $(259.6 \pm 100.2 \mathrm{mmol} / \mathrm{kg}$ and $215.1 \pm 66.9$ $\mathrm{mmol} / \mathrm{kg}$, respectively). However, it was clear that there was a great interindividual difference regarding the amount of each SCFA in both groups (Table S1). When comparing the presence of each SCFA and the amount of energy they contained, no statistically significant differences were found.

BCFA were higher in the faeces from lean volunteers $(\mathrm{p}<0.05)$. On average, faeces of lean subjects contained $7.88 \pm 2.54 \mathrm{mmol} / \mathrm{kg}$ of $i s o$-butyrate and $11.45 \pm 3.64$ $\mathrm{mmol} / \mathrm{kg}$ of $i$-valerate, while obese subjects produced on average had $3.11 \pm 1.98$ $\mathrm{mmol} / \mathrm{kg}$ of $i$-butyrate and $4.85 \pm 2.92 \mathrm{mmol} / \mathrm{kg}$ of $i$-valerate. BCFA amounts also presented a great inter-individual difference among subjects.

\section{Fermentation experiments with lean and obese microbiota}

\section{Microbial activity}

Total SCFA production was higher in fermentations using the obese microbiota when compared to the lean (Figure 1). Fermentation kinetics in terms of SCFA production observed from both inocula differed in AG, IN and control experiments. Fermentation of AG showed major differences in propionate and $n$ - 
butyrate production compared to the other fermentations, with propionate even higher than $n$-butyrate in the obese microbiota fermentations. In general, $n$ butyrate production was higher in the lean fermentations for all substrates when compared to obese, while propionate was observed to be higher in the fermentations with the obese microbiota when compared to lean.
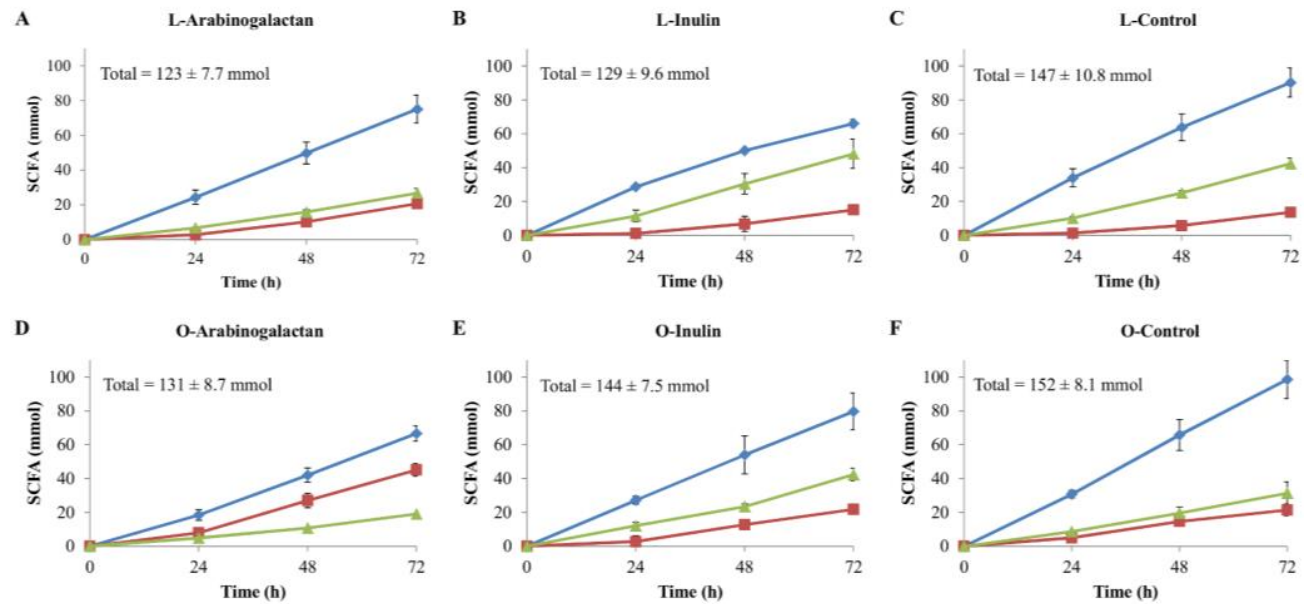

E

O-Inulin

F

o-Control
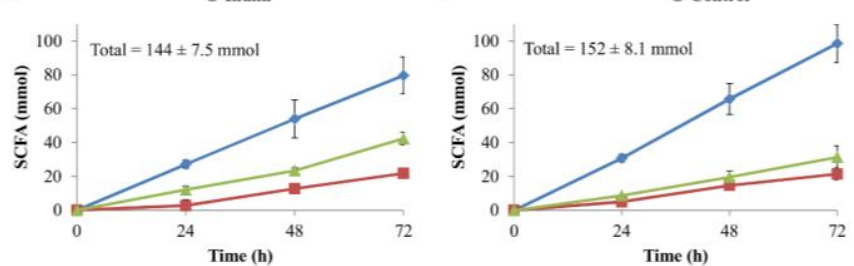

$\sim$ acetate
- -propionate
- n-butyrate

Figure 1: Cumulative production of SCFA (mmol) during the $72 \mathrm{~h}$ of fermentation of the tested substrates.

Table 1 shows the cumulative production of BCFA after $72 \mathrm{~h}$ of fermentation of AG, IN and control. Lean microbiota produced more BCFA from AG fermentation and less from IN than control. For AG this was also observed in the obese microbiota. The values from the obese fermentations were lower when compared to lean for control and AG, but not for IN.

Table 1: Cumulative production of BCFA (mmol) after $72 \mathrm{~h}$ of fermentation of AG, IN and control.

\begin{tabular}{cllcccc}
\hline & \multicolumn{3}{c}{ Lean } & & Obese \\
\cline { 2 - 7 } & $i$-butyrate & $i$-valerate & Total & $i$-butyrate & $i$-valerate & Total \\
\hline AG & $1.62 \pm 0.66$ & $2.32 \pm 0.08$ & $3.94 \pm 0.75$ & $0.55 \pm 0.36$ & $1.59 \pm 0.38$ & $2.13 \pm 0.74$ \\
\hline IN & $0.22 \pm 0.2$ & $1.23 \pm 0.15$ & $1.45 \pm 0.35$ & $0.45 \pm 0.07$ & $1.49 \pm 0.08$ & $1.94 \pm 0.15$ \\
\hline Control & $0.74 \pm 0.14$ & $1.99 \pm 0.4$ & $2.73 \pm 0.27$ & $0.44 \pm 0.28$ & $1.22 \pm 0.06$ & $1.66 \pm 0.22$ \\
\hline
\end{tabular}




\section{Energy extraction}

The microbiota from obese volunteers fermenting AG and IN extracted (slightly) more energy when compared to the lean fermentations, in accordance with the higher SCFA production. Controls remained quite similar with respect to energy extraction (Figure 2).

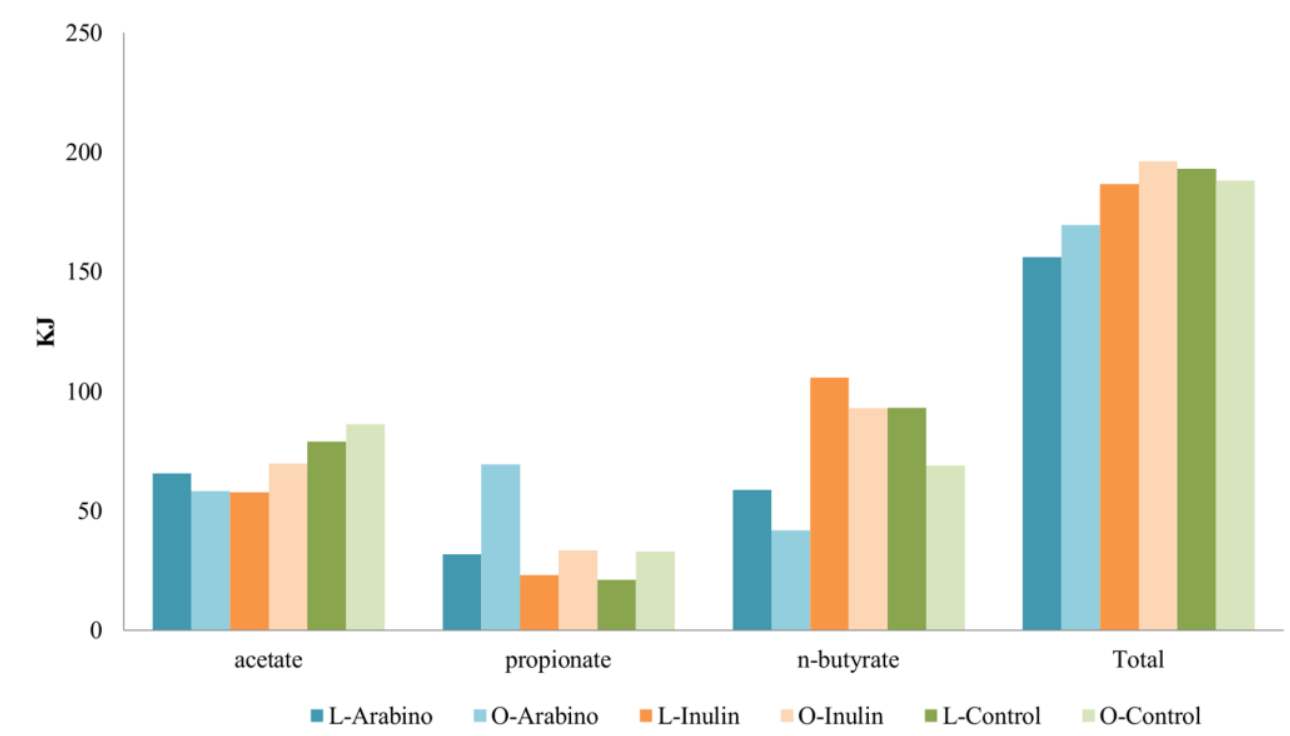

Figure 2: Energy extraction (kJ) - Values for the individual SCFA and the sum (total) obtained after $72 \mathrm{~h}$ fermentation experiments of AG, IN and control using lean or obese microbiota.

\section{Compositional changes}

By calculating L/O ratios (at the genus level), it was found that the lean microbiota had an increased relative abundance of Faecalibacterium ( 75 fold), Dorea (30 fold), Roseburia ( 6 fold), Blautia (3 fold) when compared to the obese microbiota (Table S2; for more information about the starting inocula refer to Figure S1).

The fermentation of AG and IN resulted in the growth or decrease of specific genera. Table 2 (AG) and Table 3 (IN) show the effects observed in the different microbiotas per substrate tested, compared to the control. 
Table 2: Relative change of bacterial genera after $72 \mathrm{~h}$ of fermentation experiments of arabinogalactan in TIM-2 compared to control.

\begin{tabular}{|l|c|c|}
\hline \multirow{2}{*}{ Genus } & \multicolumn{2}{c|}{ Arabinogalactan } \\
\cline { 2 - 3 } & Lean & Obese \\
\hline Lactobacillus & 0.49 & 2.56 \\
\hline Enterococcus & 0.51 & 1.65 \\
\hline Unclassified & 0.99 & 2.24 \\
\hline Escherichia & 1.11 & 0.98 \\
\hline Unclassified Clostridiaceae & 0.37 & 1.29 \\
\hline Unclassified Lactobacillaceae & 0.25 & 3.70 \\
\hline Unclassified Enterococcaceae & 0.55 & 1.39 \\
\hline Shigella & 0.57 & 1.57 \\
\hline Faecalibacterium & 0.35 & 1.53 \\
\hline Bacteroides & 1.33 & 20.47 \\
\hline Eubacterium & 0.54 & 2.95 \\
\hline Unclassified Enterobacteriaceae & 2.78 & 1.18 \\
\hline Unclassified Eubacteriaceae & 0.86 & 137.78 \\
\hline Weissella & 74.12 & 0.00 \\
\hline Collinsella & 1.63 & 0.01 \\
\hline Pseudomonas & 0.88 & 1.34 \\
\hline Blautia & 0.84 & 1.95 \\
\hline Turicibacter & 0.21 & 1.58 \\
\hline Dorea & 0.95 & 14.16 \\
\hline Unclassified Bacteroidaceae & 1.19 & 13.97 \\
\hline Fusicatenibacter & 0.03 & 16.98 \\
\hline Parabacteroides & 0.31 & 10.11 \\
\hline Unclassified Peptostreptococcaceae & 0.65 & 3.22 \\
\hline & & \\
\hline
\end{tabular}


Table 3: Relative change of bacterial genera after $72 \mathrm{~h}$ of fermentation experiments of inulin in TIM-2 compared to control.

\begin{tabular}{|l|c|c|}
\hline \multirow{2}{*}{\multicolumn{1}{c|}{ Genus }} & \multicolumn{2}{c|}{ Inulin } \\
\cline { 2 - 3 } & Lean & Obese \\
\hline Bifidobacterium & 0.77 & 1.96 \\
\hline Lactobacillus & 0.15 & 1.08 \\
\hline Unclassified & 0.83 & 2.57 \\
\hline Unclassified Bifidobacteriaceae & 1.09 & 1.83 \\
\hline Unclassified Lactobacillaceae & 0.15 & 1.57 \\
\hline Faecalibacterium & 0.97 & 2.70 \\
\hline Bacteroides & 2.84 & 0.89 \\
\hline Collinsella & 1.10 & 0.36 \\
\hline Unclassified Ruminococcaceae & 0.98 & 3.27 \\
\hline Unclassified Coriobacteriaceae & 9.06 & 0.69 \\
\hline Blautia & 0.49 & 4.53 \\
\hline Unclassified Bacteroidaceae & 2.62 & 0.88 \\
\hline Fusicatenibacter & 0.21 & 27.99 \\
\hline Unclassified Lachnospiraceae & 0.73 & 2.50 \\
\hline
\end{tabular}

\section{Arabinogalactan}

Genera that increased after the fermentation of AG by the obese microbiota but decreased in the experiments with the lean microbiota are the well-studied: Lactobacillus (3 vs 0.49 fold), Dorea (14 vs 0.95 fold), Fusinibacter (17 vs 0.03 fold), Parabacteroides (10 vs 0.31 fold), Faecalibacterium ( 2 vs 0.35 fold), and Blautia (2 vs 0.84 fold). At the species level the growth of $B$. longum was stimulated in the fermentation with the lean microbiota while it decreased in the obese ( 2 vs 0.21 fold, respectively; Table S3) this effect was opposite for $L$. mucosae ( 0.50 vs 2 fold, respectively). When compared to the control, $B$. caccae and $B$. thetaiotaomicron notably increased in the lean microbiota ( 44 and 7 fold, respectively) whilst the growth of L. gasseri (12 fold) was stimulated in the fermentations with the obese microbiota.

\section{Inulin}

After the fermentation of IN by the obese microbiota an increase in Bifidobacterium (2 vs 0.8 fold), Faecalibacterium ( 3 vs 0.97 fold), Blautia (5 vs 0.5 fold) and Fusicatenibacter (28 vs 0.2 fold) was observed with respect to the lean microbiota. The fermentation of IN promoted the growth of B. adolescentis and unclassified Bifidobacterium in the obese microbiota when compared to lean ( 5 vs 0.66 fold; 2 vs 0.76 fold, respectively; Table S3). Enterococcus faecalis was increased in the fermentations with the lean microbiota when compared to control (6 fold). 


\section{Modulatory effect of the substrates on activity and composition of the microbiota}

Species in general were found to be divided in two groups: species that were positively correlated with $n$-butyrate (found in 0 -inulin and L-inulin experiments) and species that were negatively correlated to acetate, propionate and BCFA production (found in L-Inulin and L-arabino). More specifically, correlation analysis performed showed a significant correlation of $B$. adolescentis, unclassified Bifidobacterium, $F$. prausnitzii, an unclassified Faecalibacterium and an unclassified Eubacterium with $n$-butyrate production; acetate, propionate and BCFA production were also significantly correlated with F. prausnitzii and unclassified Faecalibacterium but with an unclassified Bacteroides as well (Figure 3).

\author{
Clostridium butyricum \\ Bifidobacterium adolescentis \\ unclassified Clostridium \\ unclassified Bifidobacterium \\ Lactobacillu mucosae \\ unclassified Lactobacillus \\ Unclassified \\ Bifidobacterium longum \\ Enterococcus faecium \\ unclassified Enterococcus \\ Faecalibacterium prausnitzii \\ unclassified Bacteroides \\ unclassified Faecalibacterium \\ Bacteroides vulgatus \\ Bacteroides caccae \\ unclassified Eubacterium
}
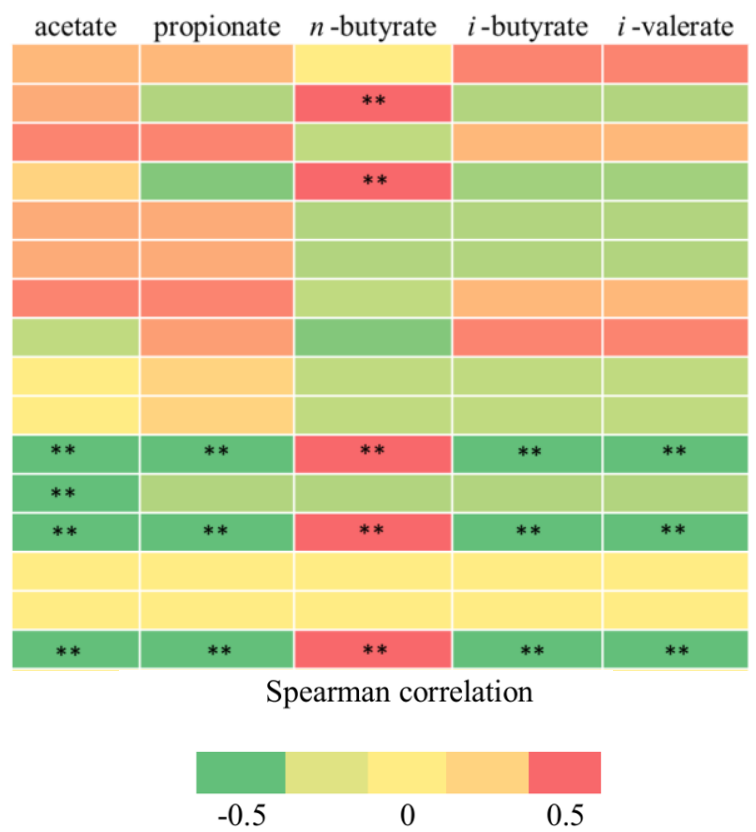

Figure 3: Correlation of metabolites and a subset of marker bacterial species

Rows correspond to bacterial species; columns correspond to measured metabolites. Red and green denote positive and negative correlation, respectively. The intensity of the colours represents the degree of association between taxa abundances and metabolites as measured by Spearman's correlations.

** indicate associations significant at the 0.01 level (2-tailed). 


\section{Discussion}

The addition of fibre to food products has been proposed to reduce the caloric density and glycaemic impact of meals (36). Therefore, there is growing interest in the use of functional fibres in the form of food ingredients, additives or supplements in order to fortify the Western diet without compromising the palatability of the food, especially in long-term weight management programs $(36,37)$.

Other properties of dietary fibre have been well documented and it has been observed that it exerts a wide array of biochemical, neurohormonal and microbiological effects in the human body (36). Here the gut microbiota, as a metabolic organ, has been found to be influenced by fibre consumption.

The amount and type of dietary fibres consumed have a direct impact on the microbial fermentation capacity (38). In this respect, studies have found that the gut microbiota from obese subjects could be more efficient in extracting energy from diet than lean subjects $(11,39,40)$. However, findings are controversial and it has been proposed that fermentation of fibres, and consequently their health effects, may be substrate dependent. Substrate dependency could be explained by the fact that dietary fibre reflects a heterogeneous group of compounds that differ in their chemical structure and physico-chemical properties, therefore reflecting on different physiological functions or health benefits $(41,42)$.

In this study, AG and IN were provided to the microbiota from lean or obese subjects and $72 \mathrm{~h}$ fermentation experiments were performed. The aim was to compare the differences in the fermentation profiles of these two fibres with respect to microbiota composition, but particularly with respect to production of the microbial metabolites SCFA, which are an energy source for the host. Determination of fibre fermentation in humans and rats is a common approach to characterize the capacity of the gut microbiota to ferment a specific substrate. However, these studies are expensive, time consuming and in the case of rats the metabolic products yielded from the fermentation of several types of fibre have been found to be significantly lower when compared to humans (43-45). Moreover, studies in humans are limited because of the limited sampling capacity (non-invasively only faeces can be collected), while most of the SCFA produced are taken up during transit of the chyme through the colon (estimated at $95 \%$ of produced SCFA) (46). Here, we used the TIM- 2 system as an alternative tool to animal and human studies. In vitro systems simulating the large intestine have been validated and found to accurately predict the fermentation of fibres in human subjects by presenting the same magnitude of the differences in SCFA production (43). In the current study pooling of faecal samples was performed to create a standardized microbiota, which was subsequently frozen and stored. 
Both pooling (27) and the impact of freezing (28) have been validated before. However, besides the lack of host interactions in such in vitro systems, another limitation is the characteristic variability of the microbiota used to inoculate the models, which was derived from a group of donors.

Involving different participants in a study like the present one constitutes one of the factors that influence the sometimes contradictory results in gut microbiota research. Still, the participants recruited for this study were considered according to their diet, consumption of prebiotics, probiotics and medication as specified in the M\&M section. We considered including the microbiota from only those volunteers who fitted the best for the requirements from the two groups that we studied (i.e. lean and obese).

Importantly, BMI index was prioritized given that the main goal of this investigation was exploring the differences between lean and obese microbiota fermenting AG and IN. We want to emphasize that the BMI index among subjects was very similar. This was reflected by the low standard deviation value observed in both groups (M\&M). By setting the inclusion requirements mentioned above we believe that the outcome of our studies is the result of a lean or obese microbiome phenotype. Still, we acknowledge that the age difference from our group of volunteers might have contributed to differences in the microbiota.

The current study provides more evidence for the consumption of specific ingredients with the aim of modulating the gut microbiota in the context of obesity.

\section{Impact of the substrates on metabolic activity}

When compared against each other and against the control, both fibres presented different fermentation kinetics (in terms of SCFA production) (Figure 1). The difference between the production of propionate and $n$-butyrate in AG experiments using both microbiotas (Figure S2) is interesting in the light of the discussion of the impact of microbial metabolites in obesity. The increase in propionate production in the experiments with the obese microbiota suggests that via this metabolite AG could be protective against inflammation and promote satiety in obese subjects.

Despite that most studies about the anti-inflammatory role of SCFA have been focused on the effects of $n$-butyrate and acetate $(47,48)$, there is evidence pointing to propionate as a metabolite with a strong role against inflammation (49). On the one hand, propionate acts as a ligand of G-protein-couple receptors (GPCR) 41 and 43 (50). These receptors when activated, induce an increase of GLP-1 (which slows down gastric emptying and promotes satiety) and PYY 
(which up-regulates food digestion and absorption). Besides this, it has also been shown that when they are absent (at least for GPCR43 in knockout mice) there is an exacerbated inflammation in inflammatory-disease models (51). Moreover, propionate has been found to decrease fatty acid levels in plasma (47). As there is also evidence indicating that high plasma levels of fatty acids cause inflammation and consequently insulin resistance, the postulation of propionate as a molecule with anti-obesity properties is reinforced.

On the other hand, propionate has also been linked to autism (52) and hence, an increase in this metabolite may not be desirable, although the mechanism is not entirely clear.

In this study, IN was found to increase the production of $n$-butyrate in the fermentations with the lean microbiota (Figure S2) when compared to the obese. The butyrogenic effect of IN has been previously observed in vivo and in vitro (17,18,53-57). $n$-Butyrate has been postulated as a molecule with health benefits for the human host since it has been found to be an important source of energy for colonocytes, with a potential protective role against colon cancer. In addition, it has been inversely correlated with inflammatory bowel diseases such as Crohn's disease (58-61). To our knowledge only two studies have tested in vitro the fermentation of IN in both microbiotas (lean and obese). Both Sarbini et al (17) and Bussolo de Souza et al (18) found that the obese fermentation of IN produced higher concentrations of $n$-butyrate when compared to the lean fermentation. The inocula composition in these experiments is not the same (Table S2) and that may have contributed to our results. Nevertheless, we have observed that there are some compositional similarities in both inocula despite being prepared in different years (Table S2).

The fermentation of AG showed decreased BCFA concentrations in the fermentations with the obese microbiota when compared the lean. This supports the work from Vince and colleagues (62) and Robinson \& Slavin (20) who observed a significant decreased in products from proteolytic fermentation (specifically ammonia) by intestinal bacteria after the supplementation of AG. However, our study brings evidence, for the first time, about this beneficial effect of AG in obese subjects.

Though production of BCFA was not lower in the obese microbiota fermenting IN when compared to lean, at least, when it is compared to control, the fermentation of IN with the lean microbiota is lower, giving also a good indication of the prebiotic effect of IN in ameliorating proteolytic fermentation. This effect has been previously observed in vitro as well as in vivo $(29,63,64)$. 


\section{Energy extraction}

The hypothesis that the gut microbiota in obese individuals facilitates the additional extraction of calories from diet has been previously reviewed $(38,65,66)$. In this study we were not able to confirm that the energy yield (in terms of SCFA produced) was higher in the faeces from the obese donors in comparison with the lean donors (Table S1). However, the absorption of SCFA has been described as a very efficient process since only $5-10 \%$ is excreted in the faeces (67). Furthermore, in a study performed by Jumpertz and colleagues (68) overfeeding in lean subjects was associated with a greater decrease in stool energy loss showing a relation between loss of energy in faeces and energy load. Therefore, based on our results we could hypothesize that obese individuals may have higher capacity of absorbing SCFA in their gut and, therefore, may have lower amounts of these metabolites in their faeces compared with lean subjects. However, we found a substrate dependent effect on the metabolic activity and consequently energy extraction when fermentations of both AG and IN were performed. These findings confirm our previous observations where a higher amount of energy extracted from the fermentation of IN was also found after fermentation by obese microbiota when compared to lean (18). Although the difference is small, over a prolonged period this may add up to several kilos of body weight, since, as previously mentioned, an elevated production of SCFA contributes to a higher energy input to the host. But at the same time these metabolites have also been found to present satiety-enhancing properties via the activation of GPCRs. Thus, it could be that the enhancement of the production of these metabolites could be protective against obesity in this population, despite their energy content. Still, more research is needed in this area.

\section{Compositional changes}

When the inocula prepared from both lean and obese subjects (previous study; 2012) is compared to the inocula from the present study (2014), we observed some compositional differences in the shared bacterial groups. This shows that not all inocula are the same. However, there are some similarities in some increased groups observed from the $\mathrm{L} / 0$ ratios (Table S2) making it possible to distinguish certain bacteria belonging for example to Gemmiger, Dorea, Roseburia, Alistipes genera which both times were highly abundant in the lean microbiota and deserve being more investigated about their potential role in leanness.

After fermenting AG by the different microbiota, the growth of some groups of bacteria was highly stimulated in one microbiota whilst they decreased in the other. Between the groups that were benefited from the fermentation of AG in 
the obese microbiota we found Faecalibacterium, Dorea and Blautia. Judging from the ratios calculated in order to compare both inocula (L/O 2014; Table S2), it seems that the community from obese donors tended to re-structure towards the microbiota from lean donors after the fermentation of AG (Table 2).

This effect was also previously observed by Bussolo de Souza et al (18) and Condezo-Hoyos et al (19) when testing the prebiotic effects of cassava bagasse and different apple cultivars on the composition from lean and obese microbiota. Lactobacillus was observed to increase in the obese microbiota fermenting AG (Table 2). Furthermore, we also described an increase of the species L. mucosae and L. gasseri in the obese microbiota after fermenting AG. Our results are in agreement with Robinson \& Slavin (20) who showed that a diet supplemented with AG increased the concentration of Lactobacillus in the faeces from healthy participants. In addition, a study conducted by Santacruz et al (69) showed a parallel reduction of body mass index (BMI) and increase of Lactobacillus spp. amounts in obese adolescents suggesting a potential role of this genera in obesity and body weight control. Based on these findings we suggest that our study brings more evidence about how a prebiotic such as AG can beneficially influence the composition of the microbiota from obese subjects in weight management. The lean microbiota fermenting AG presented an increase in B. thetaiotaomicron. The growth of this species on AG has also been found by others (70). $B$. thetaiotaomicron colonization has been observed to elicit gene expression involved with the fortification of the intestinal barrier function and the maintenance of mucosal integrity which may suggest to be especially beneficial in obese subjects (71).

The obese microbiota composition on IN shifted the simulated gut environment into a healthier milieu with increases of beneficial bacteria belonging to the Faecalibacterium, Blautia, Fusicatenibacterium and Bifidobacterium genera. The bifidogenic effect of IN was demonstrated to be more pronounced in the obese microbiota when compared to lean (Table 3). Interestingly, IN was shown to selectively stimulate the growth of $B$. adolescentis (Table S4). This is in agreement with the upregulation of $B$. adolescentis by IN also found by Ramirez-Farias et al (23) and B. animalis by Venema and Maathuis (72). In fact, the latter authors hypothesized that within the Bifidobacterium genus, the diversity was diminished by inulin. This was also observed to some degree in our experiments (Table S4).

The difference in the bifidogenic effect of IN in the obese microbiota, especially in the case of $B$. adolescentis growth, can be explained by $i$ ) a long-known inverse relation between initial amounts present and the observed increase (73). As observed e.g. by Korpela et al (74) and other authors, the lower the abundance 
of Bifidobacterium spp. at the starting point of an intervention, the more the increase observed after the administration of an specific prebiotic, and vice versa (74-76); or ii) the high specificity of IN for stimulating the growth of certain bifidobacterial species as observed by Venema \& Maathuis (72). A high abundance of bifidobacteria could be protective in obesity since it is speculated that this bacteria may decrease pro-inflammatory cytokines and decrease endotoxaemia which can improve glucose-induced insulin and glucose tolerance $(17,77)$.

\section{Conclusion}

First line strategies to combat obesity include exercise and/or a balanced dietary regime. Though apparently simple, such changes in people's life are difficult to maintain and in most of the cases, patients struggle to follow these recommendations. Due to the complexity of this condition, it is vital to identify weight loss methods by which subjects can successfully achieve long-term results. In this respect, using fibres is a potential tool to supplement diet in weight management due to their satiety aspects, as well as in modulating the gut microbiota. Here in this study we have identified the potential of arabinogalactan and inulin in stimulating a gut community more related to a lean profile. Metabolically, arabinogalactan fermentation showed a higher production of propionate when compared to $n$-butyrate in the obese microbiota fermentations. In general, lean microbiota produced more $n$-butyrate from the fermentation of both substrates when compared to the obese microbiota. This would be interesting to investigate in light of the potential activation of different GPCRs by these SCFA. However, these effects should be more studied in humans focusing especially on the role of these fibres in satiety.

\section{Acknowledgments}

The authors thank Freddy J. Troost and Bouke Salden for facilitating the recruitment of the donors, and declare that there is no conflict of interest.

\section{Author contributions}

Conceived and designed the experiments: MA, KV. Performed the experiments: MA. Analysed the data: MA, CBS, KV. Contributed reagents/materials/analysis tools: MA, CBS, KV. Wrote the paper: MA, CBS, KV. 


\section{References}

1. Yang J, Keshavarzian A, Rose DJ. Impact of dietary fiber fermentation from cereal grains on metabolite production by the fecal microbiota from normal weight and obese individuals. J Med Food. 2013 Sep;16(9):862-7.

2. Elli M, Colombo 0, Tagliabue A. A common core microbiota between obese individuals and their lean relatives? Evaluation of the predisposition to obesity on the basis of the fecal microflora profile. Med Hypotheses. 2010;75(4):350-2.

3. Lozupone CA, Stombaugh JI, Gordon JI, Jansson JK, Knight R. Diversity, stability and resilience of the human gut microbiota. Nature. 2012 Sep;489(7415):220-30.

4. Topping DL, Clifton PM. Short-chain fatty acids and human colonic function: roles of resistant starch and nonstarch polysaccharides. Physiol Rev. 2001;81(3):1031-64.

5. Russell WR, Gratz SW, Duncan SH, Holtrop G, Ince J, Scobbie L, et al. Highprotein, reduced-carbohydrate weight-loss diets promote metabolite profiles likely to be detrimental to colonic health. Am J Clin Nutr. 2011 May;93(5):1062-72.

6. Freeland KR, Wolever TMS. Acute effects of intravenous and rectal acetate on glucagon-like peptide-1, peptide YY, ghrelin, adiponectin and tumour necrosis factor-alpha. Br J Nutr. 2010 Feb;103(3):460-6.

7. Tarini J, Wolever TMS. The fermentable fibre inulin increases postprandial serum short-chain fatty acids and reduces free-fatty acids and ghrelin in healthy subjects. Appl Physiol Nutr Metab = Physiol Appl Nutr Metab. 2010 Feb;35(1):9-16.

8. Anderson JW, Baird P, Davis RHJ, Ferreri S, Knudtson M, Koraym A, et al. Health benefits of dietary fiber. Nutr Rev. 2009 Apr;67(4):188-205.

9. Liu S, Willett WC, Manson JE, Hu FB, Rosner B, Colditz G. Relation between changes in intakes of dietary fiber and grain products and changes in weight and development of obesity among middle-aged women. Am J Clin Nutr. 2003 Nov;78(5):920-7.

10. Royall D, Wolever TM, Jeejeebhoy KN. Clinical significance of colonic fermentation. Am J Gastroenterol. 1990 Oct;85(10):1307-12.

11. Turnbaugh PJ, Ley RE, Mahowald MA, Magrini V, Mardis ER, Gordon JI. An obesity-associated gut microbiome with increased capacity for energy harvest. Nature. 2006;444(7122):1027-31.

12. Schwiertz A, Taras D, Schafer K, Beijer S, Bos NA, Donus C, et al. Microbiota and SCFA in lean and overweight healthy subjects. Obes (Silver Spring). 2010;18(1):190-5. 
13. Aguirre M, Jonkers DMAE, Troost FJ, Roeselers G, Venema K. In vitro characterization of the impact of different substrates on metabolite production, energy extraction and composition of gut microbiota from lean and obese subjects. PLoS One. 2014;9(11):e113864.

14. Turnbaugh PJ, Hamady M, Yatsunenko T, Cantarel BL, Duncan A, Ley RE, et al. A core gut microbiome in obese and lean twins. Nature. 2009 Jan;457(7228):480-4.

15. Payne AN, Chassard C, Zimmermann M, Muller P, Stinca S, Lacroix C. The metabolic activity of gut microbiota in obese children is increased compared with normal-weight children and exhibits more exhaustive substrate utilization. Nutr Diabetes. 2011 Jul;1:e12.

16. Sarbini SR, Kolida S, Deaville ER, Gibson GR, Rastall RA. Potential of novel dextran oligosaccharides as prebiotics for obesity management through in vitro experimentation. Br J Nutr. 2014 Oct;112(8):1303-14.

17. Sarbini SR, Kolida S, Gibson GR, Rastall RA. In vitro fermentation of commercial alpha-gluco-oligosaccharide by faecal microbiota from lean and obese human subjects. Br J Nutr. 2013 Jun;109(11):1980-9.

18. Bussolo de Souza C, Roeselers G, Troost F, Jonkers D, Koenen ME, Venema K. Prebiotic effects of cassava bagasse in TNO's in vitro model of the colon in lean versus obese microbiota. J Funct Foods. 2014;11(C):210-20.

19. Condezo-Hoyos L, Mohanty IP, Noratto GD. Assessing non-digestible compounds in apple cultivars and their potential as modulators of obese faecal microbiota in vitro. Food Chem. 2014 Oct;161:208-15.

20. Robinson RR, Feirtag J, Slavin JL. Effects of dietary arabinogalactan on gastrointestinal and blood parameters in healthy human subjects. J Am Coll Nutr. 2001 Aug;20(4):279-85.

21. Crociani F, Alessandrini A, Mucci MM, Biavati B. Degradation of complex carbohydrates by Bifidobacterium spp. Int J Food Microbiol. 1994 Dec;24(1-2):199-210.

22. Daguet D, Pinheiro I, Verhelst A, Possemiers S, Marzorati M. Arabinogalactan and fructooligosaccharides improve the gut barrier function in distinct areas of the colon in the Simulator of the Human Intestinal Microbial Ecosystem. J Funct Foods. 2016;20(Supplement C):369-79.

23. Ramirez-Farias C, Slezak K, Fuller Z, Duncan A, Holtrop G, Louis P. Effect of inulin on the human gut microbiota: stimulation of Bifidobacterium adolescentis and Faecalibacterium prausnitzii. Br J Nutr. 2009 Feb;101(4):541-50.

24. Kim LS, Waters RF, Burkholder PM. Immunological activity of larch 
arabinogalactan and Echinacea: a preliminary, randomized, double-blind, placebo-controlled trial. Altern Med Rev. 2002 Apr;7(2):138-49.

25. Hauer J, Anderer FA. Mechanism of stimulation of human natural killer cytotoxicity by arabinogalactan from Larix occidentalis. Cancer Immunol Immunother. 1993;36(4):237-44.

26. Hotamisligil GS. Inflammation and metabolic disorders. Nature. 2006 Dec;444(7121):860-7.

27. Aguirre M, Ramiro-Garcia J, Koenen ME, Venema K. To pool or not to pool? Impact of the use of individual and pooled fecal samples for in vitro fermentation studies. J Microbiol Methods. 2014;107:1-7.

28. Aguirre M, Eck A, Koenen ME, Savelkoul PHM, Budding AE, Venema K. Evaluation of an optimal preparation of human standardized fecal inocula for in vitro fermentation studies. J Microbiol Methods. 2015 Oct;117:7884.

29. van Nuenen MHMC, Diederick Meyer P, Venema K. The effect of various inulins and Clostridium difficile on the metabolic activity of the human colonic microbiota in vitro. Microb Ecol Health Dis. 2003;15(2-3):137-44.

30. De Graaf AA, Maathuis A, de Waard P, Deutz NE, Dijkema C, de Vos WM, et al. Profiling human gut bacterial metabolism and its kinetics using [U13C]glucose and NMR. NMR Biomed. 2009/07/14. 2010;23(1):2-12.

31. Maathuis A, Hoffman A, Evans A, Sanders L, Venema K. The effect of the undigested fraction of maize products on the activity and composition of the microbiota determined in a dynamic in vitro model of the human proximal large intestine. J Am Coll Nutr. 2009;28(6):657-66.

32. van der Kamp JW, Jones J, McCleary B, Topping D. Dietary fibre: New frontiers for food and health. Wageningen Academic Publishers; 2010. $592 \mathrm{p}$.

33. Marino P, Sutin K. The ICU Book: Wolters Kluwer Health. Philadelphia, USA; 2012.

34. DeSantis TZ, Hugenholtz P, Larsen N, Rojas M, Brodie EL, Keller K, et al. Greengenes, a chimera-checked 16S rRNA gene database and workbench compatible with ARB. Appl Environ Microbiol. 2006 Jul;72(7):5069-72.

35. Cole JR, Wang Q, Fish JA, Chai B, McGarrell DM, Sun Y, et al. Ribosomal Database Project: data and tools for high throughput rRNA analysis. Nucleic Acids Res. 2014 Jan;42(Database issue):D633-42.

36. Lyon MR, Kacinik V. Is there a place for dietary fiber supplements in weight management? Curr Obes Rep. 2012 Jun;1(2):59-67.

37. Spill MK, Birch LL, Roe LS, Rolls BJ. Hiding vegetables to reduce energy density: an effective strategy to increase children's vegetable intake and 
reduce energy intake. Am J Clin Nutr. 2011;94(3):735-41.

38. Aguirre M, Venema K. Does the gut microbiota contribute to obesity? going beyond the gut feeling. Microorganisms. 2015 Apr;3(2):213-35.

39. Backhed F, Ding H, Wang T, Hooper L V, Koh GY, Nagy A, et al. The gut microbiota as an environmental factor that regulates fat storage. Proc Natl Acad Sci U S A. 2004 Nov;101(44):15718-23.

40. Turnbaugh PJ, Backhed F, Fulton L, Gordon JI. Diet-induced obesity is linked to marked but reversible alterations in the mouse distal gut microbiome. Cell Host Microbe. 2008;3(4):213-23.

41. Wanders AJ, Jonathan MC, van den Borne JJGC, Mars M, Schols HA, Feskens EJM, et al. The effects of bulking, viscous and gel-forming dietary fibres on satiation. Br J Nutr. 2013 Apr;109(7):1330-7.

42. Cummings JH, Edmond LM, Magee EA. Dietary carbohydrates and health: do we still need the fibre concept? Clin Nutr Suppl. 2004;1(2):5-17.

43. Wisker E, Daniel M, Rave G, Feldheim W. Fermentation of non-starch polysaccharides in mixed diets and single fibre sources: comparative studies in human subjects and in vitro. Br J Nutr. 1998 Sep;80(3):253-61.

44. Bach Knudsen KE, Wisker E, Daniel M, Feldheim W, Eggum BO. Digestibility of energy, protein, fat and non-starch polysaccharides in mixed diets: comparative studies between man and the rat. Br J Nutr. 1994 Apr;71(4):471-87.

45. Wisker E, Bach Knudsen KE, Daniel M, Eggum BO, Feldheim W. Energy values of non-starch polysaccharides: comparative studies in humans and rats. J Nutr. 1997 Jan;127(1):108-16.

46. Cummings JH, Beatty ER, Kingman SM, Bingham SA, Englyst HN. Digestion and physiological properties of resistant starch in the human large bowel. Br J Nutr. 1996 May;75(5):733-47.

47. Al-Lahham S, Peppelenbosch MP, Roelofsen H, Vonk RJ, Venema K. Biological effects of propionic acid in humans; metabolism, potential applications and underlying mechanisms. Biochim Biophys Acta. 2010;1801(11):1175-83.

48. Roelofsen H, Priebe MG, Vonk RJ. The interaction of short-chain fatty acids with adipose tissue: relevance for prevention of type 2 diabetes. Benef Microbes. 2010 Nov;1(4):433-7.

49. Al-Lahham S, Roelofsen H, Rezaee F, Weening D, Hoek A, Vonk R, et al. Propionic acid affects immune status and metabolism in adipose tissue from overweight subjects. Eur J Clin Invest. 2012;42(4):357-64.

50. Brown AJ, Goldsworthy SM, Barnes AA, Eilert MM, Tcheang L, Daniels D, et al. The Orphan G protein-coupled receptors GPR41 and GPR43 are 
activated by propionate and other short chain carboxylic acids. J Biol Chem. 2003 Mar;278(13):11312-9.

51. Maslowski KM, Vieira AT, Ng A, Kranich J, Sierro F, Yu D, et al. Regulation of inflammatory responses by gut microbiota and chemoattractant receptor GPR43. Nature. 2009 Oct;461(7268):1282-6.

52. MacFabe DF, Cain NE, Boon F, Ossenkopp K-P, Cain DP. Effects of the enteric bacterial metabolic product propionic acid on object-directed behavior, social behavior, cognition, and neuroinflammation in adolescent rats: Relevance to autism spectrum disorder. Behav Brain Res. 2011 Feb;217(1):47-54.

53. van de Wiele T, Boon N, Possemiers S, Jacobs H, Verstraete W. Inulin-type fructans of longer degree of polymerization exert more pronounced in vitro prebiotic effects. J Appl Microbiol. 2007 Feb;102(2):452-60.

54. Grootaert C, Van den Abbeele P, Marzorati M, Broekaert WF, Courtin CM, Delcour JA, et al. Comparison of prebiotic effects of arabinoxylan oligosaccharides and inulin in a simulator of the human intestinal microbial ecosystem. FEMS Microbiol Ecol. 2009 Aug;69(2):231-42.

55. Hughes SA, Shewry PR, Li L, Gibson GR, Sanz ML, Rastall RA. In vitro fermentation by human fecal microflora of wheat arabinoxylans. J Agric Food Chem. 2007 May;55(11):4589-95.

56. Sakaguchi E, Sakoda C, Toramaru Y. Caecal fermentation and energy accumulation in the rat fed on indigestible oligosaccharides. Br J Nutr. 1998 Nov;80(5):469-76.

57. Juskiewicz J, Zdunczyk Z, Frejnagel S. Caecal parameters of rats fed diets supplemented with inulin in exchange for sucrose. Arch Anim Nutr. 2007 Jun;61(3):201-10.

58. Sokol H, Pigneur B, Watterlot L, Lakhdari O, Bermudez-Humaran LG, Gratadoux J-J, et al. Faecalibacterium prausnitzii is an anti-inflammatory commensal bacterium identified by gut microbiota analysis of Crohn disease patients. Proc Natl Acad Sci U S A. 2008 Oct;105(43):16731-6.

59. Velazquez OC, Lederer HM, Rombeau JL. Butyrate and the colonocyte. Production, absorption, metabolism, and therapeutic implications. Adv Exp Med Biol. 1997;427:123-34.

60. Willing B, Halfvarson J, Dicksved J, Rosenquist M, Järnerot G, Engstrand L, et al. Twin studies reveal specific imbalances in the mucosa-associated microbiota of patients with ileal Crohn's disease. Inflamm Bowel Dis. 2009;15(5):653-60.

61. Basson MD, Turowski GA, Rashid Z, Hong F, Madri JA. Regulation of human colonic cell line proliferation and phenotype by sodium butyrate. Dig Dis 
Sci. 1996 Oct;41(10):1989-93.

62. Vince AJ, McNeil NI, Wager JD, Wrong OM. The effect of lactulose, pectin, arabinogalactan and cellulose on the production of organic acids and metabolism of ammonia by intestinal bacteria in a faecal incubation system. Br J Nutr. 1990 Jan;63(1):17-26.

63. De Preter V, Falony G, Windey K, Hamer HM, De Vuyst L, Verbeke K. The prebiotic, oligofructose-enriched inulin modulates the faecal metabolite profile: an in vitro analysis. Mol Nutr Food Res. 2010 Dec;54(12):1791801.

64. De Preter V, Vanhoutte T, Huys G, Swings J, De Vuyst L, Rutgeerts P, et al. Effects of Lactobacillus casei Shirota, Bifidobacterium breve, and oligofructose-enriched inulin on colonic nitrogen-protein metabolism in healthy humans. Am J Physiol Gastrointest Liver Physiol. 2007 Jan;292(1):G358-68.

65. DiBaise JK, Zhang H, Crowell MD, Krajmalnik-Brown R, Decker GA, Rittmann BE. Gut microbiota and its possible relationship with obesity. Mayo Clin Proc. 2008;83(4):460-9.

66. Tilg H. Obesity, metabolic syndrome, and microbiota: multiple interactions. J Clin Gastroenterol. 2010 Sep;44 Suppl 1:S16-8.

67. De Graaf A, Venema K. Gaining insight into microbial physiology in the large intestine: a special role for stable isotopes. 2007;53:73-314.

68. Jumpertz R, Le DS, Turnbaugh PJ, Trinidad C, Bogardus C, Gordon JI, et al. Energy-balance studies reveal associations between gut microbes, caloric load, and nutrient absorption in humans. Am J Clin Nutr. 2011;94(1):5865.

69. Santacruz A, Marcos A, Warnberg J, Marti A, Martin-Matillas M, Campoy C, et al. Interplay between weight loss and gut microbiota composition in overweight adolescents. Obesity (Silver Spring). 2009 Oct;17(10):190615.

70. Macfarlane GT, Macfarlane S, Gibson GR. Co-culture of Bifidobacterium adolescentis and Bacteroides thetaiotaomicron in arabinogalactan-limited chemostats: effects of dilution rate and pH. Anaerobe. 1995 0ct;1(5):27581.

71. Zocco MA, Ainora ME, Gasbarrini G, Gasbarrini A. Bacteroides thetaiotaomicron in the gut: molecular aspects of their interaction. Dig Liver Dis. 2007 Aug;39(8):707-12.

72. Venema K, Maathuis AJH. A PCR-based method for identification of bifidobacteria from the human alimentary tract at the species level. FEMS Microbiol Lett. 2003 Jul;224(1):143-9. 
73. Roberfroid MB. Prebiotics: preferential substrates for specific germs? Am J Clin Nutr. 2001 Feb;73(2 Suppl):406S-409S.

74. Korpela K, Flint HJ, Johnstone AM, Lappi J, Poutanen K, Dewulf E, et al. Gut microbiota signatures predict host and microbiota responses to dietary interventions in obese individuals. PLoS One. 2014;9(6):e90702.

75. Davis LMG, Martinez I, Walter J, Hutkins R. A dose dependent impact of prebiotic galactooligosaccharides on the intestinal microbiota of healthy adults. Int J Food Microbiol. 2010 Dec;144(2):285-92.

76. Kolida S, Meyer D, Gibson GR. A double-blind placebo-controlled study to establish the bifidogenic dose of inulin in healthy humans. Eur J Clin Nutr. 2007 Oct;61(10):1189-95.

77. Cani PD, Neyrinck AM, Fava F, Knauf C, Burcelin RG, Tuohy KM, et al. Selective increases of bifidobacteria in gut microflora improve high-fatdiet-induced diabetes in mice through a mechanism associated with endotoxaemia. Diabetologia. 2007;50(11):2374-83. 


\section{Supplemental material}

Table S1: Metabolites and energy extraction (in terms of SCFA) measured in the faeces from the volunteers.

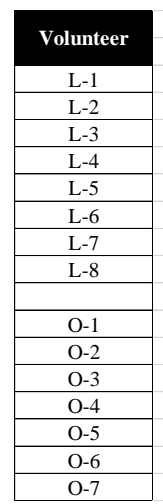

\begin{tabular}{|c|c|c|c|}
\hline \multicolumn{5}{|c|}{ SCFA (mmol/kg) } \\
\hline acetate & propionate & n-butyrate & Total \\
\hline 233.3 & 74.9 & 49.5 & 377.7 \\
\hline 219.7 & 58.1 & 64.8 & 342.6 \\
\hline 232.3 & 44.6 & 36.4 & 313.3 \\
\hline 83.6 & 34.1 & 26.5 & 144.2 \\
\hline 85.9 & 15.9 & 18.7 & 120.6 \\
\hline 89.5 & 33.0 & 37.8 & 160.2 \\
\hline 168.3 & 66.2 & 58.7 & 293.2 \\
\hline 205.8 & 69.3 & 69.6 & 344.8 \\
\hline & & & \\
\hline 138.6 & 43.1 & 35.1 & 219.9 \\
\hline 176.8 & 92.1 & 54.2 & 323.0 \\
\hline 112.2 & 32.8 & 34.3 & 179.3 \\
\hline 83.0 & 22.7 & 20.4 & 126.1 \\
\hline 157.2 & 37.8 & 39.3 & 234.3 \\
\hline 180.9 & 44.4 & 40.6 & 265.9 \\
\hline 109.5 & 30.0 & 20.4 & 159.9 \\
\hline \multicolumn{4}{|c}{} \\
\hline
\end{tabular}

\begin{tabular}{|c|c|c|}
\hline \multicolumn{3}{|c|}{ BCFA (mmol/kg) } \\
\hline i-butyrate & i-valerate & Total \\
\hline 11.4 & 16.3 & 27.7 \\
\hline 7.89 & 10.0 & 17.9 \\
\hline 8.00 & 11.1 & 19.1 \\
\hline 4.57 & 6.91 & 11.5 \\
\hline 3.84 & 6.05 & 9.89 \\
\hline 8.30 & 12.7 & 21.0 \\
\hline 9.60 & 14.2 & 23.8 \\
\hline 9.42 & 14.3 & 23.7 \\
\hline & & \\
\hline 4.17 & 6.11 & 10.3 \\
\hline 0.00 & 1.62 & 1.62 \\
\hline 2.4 & 2.87 & 5.27 \\
\hline 2.78 & 4.17 & 6.96 \\
\hline 5.63 & 9.31 & 14.9 \\
\hline 5.07 & 7.64 & 12.7 \\
\hline 1.73 & 2.19 & 3.92 \\
\hline \multicolumn{2}{|c|}{} \\
\end{tabular}

\begin{tabular}{|c|c|c|c|}
\hline \multicolumn{4}{|c|}{ Energy extraction $(\mathbf{K J} / \mathbf{k g})$} \\
\hline acetate & propionate & n-butyrate & Total \\
\hline 204.2 & 115.1 & 108.4 & 427.6 \\
\hline 192.0 & 89.1 & 139.7 & 423.4 \\
\hline 203.3 & 68.6 & 79.9 & 351.5 \\
\hline 73.2 & 52.3 & 58.2 & 183.7 \\
\hline 75.3 & 24.5 & 41.1 & 140.6 \\
\hline 78.2 & 50.6 & 82.8 & 211.7 \\
\hline 147.3 & 101.7 & 128.9 & 377.4 \\
\hline 179.9 & 106.7 & 152.7 & 438.9 \\
\hline & & & \\
\hline 121. & 66.1 & 77.0 & 264.4 \\
\hline 154.4 & 141.4 & 118.8 & 414.6 \\
\hline 98.3 & 50.2 & 75.3 & 223.8 \\
\hline 72.8 & 34.9 & 44.8 & 152.3 \\
\hline 137.7 & 58.2 & 86.2 & 281.6 \\
\hline 158.2 & 68.2 & 89.1 & 315.5 \\
\hline 95.8 & 46.0 & 44.8 & 186.6 \\
\hline
\end{tabular}

Table S2: Ratio L/O. Relative ratio of bacterial genera different between lean and obese at t0 in TIM-2 in the current study and in a study by Bussolo et al. (18) in 2012.

\begin{tabular}{|l|c|c|}
\multicolumn{1}{c}{ Genera } & $\mathbf{2 0 1 4}$ & $\mathbf{2 0 1 2}$ \\
\hline Bifidobacterium & 2.33 & 1.05 \\
\hline Lactobacillus & 0.78 & 0.07 \\
\hline Enterococcus & 0.85 & 0.31 \\
\hline Lactococcus & 53.63 & 0.24 \\
\hline Shigella & 0.21 & 0.34 \\
\hline Streptococcus & 20.01 & 0.35 \\
\hline Faecalibacterium & 74.82 & 680.58 \\
\hline Bacteroides & 2.81 & 3.99 \\
\hline Weissella & 2.09 & 0.99 \\
\hline Collinsella & 0.76 & 6.60 \\
\hline Gemmiger & 23.77 & 11.87 \\
\hline Blautia & 2.93 & 58.96 \\
\hline Roseburia & 5.64 & 205.17 \\
\hline Dorea & 30.39 & 17.83 \\
\hline Coprobacillus & 1.12 & 7.92 \\
\hline unclassified Erysipelotrichaceae & 0.16 & 14.14 \\
\hline Parabacteroides & 0.55 & 19.13 \\
\hline Anaerostipes & 1.30 & 131.47 \\
\hline Alistipes & 3.91 & 59.01 \\
\hline
\end{tabular}


Chapter 6

Table S3: Relative change of bacterial species after $72 \mathrm{~h}$ of fermentation experiments of arabinogalactan and inulin in TIM-2 using microbiota from lean and obese individuals.

\begin{tabular}{|c|c|c|c|c|}
\hline Species & L-Arabino & 0-Arabino & L-Inulin & 0-Inulin \\
\hline Clostridium butyricum & 0.40 & 0.24 & 0.19 & 0.30 \\
\hline Bifidobacterium adolescentis & 0.01 & & 0.66 & 4.68 \\
\hline unclassified Clostridium & 0.41 & 0.80 & 0.28 & 0.62 \\
\hline unclassified Bifidobacterium & 0.17 & 0.07 & 0.76 & 1.90 \\
\hline Lactobacillus mucosae & 0.50 & 2.06 & 1.08 & \\
\hline unclassified Lactobacillus & 0.58 & 3.14 & 0.71 & \\
\hline Unclassified & 1.03 & 2.29 & 0.89 & 2.02 \\
\hline Bifidobacterium longum & 2.00 & 0.21 & 0.15 & 0.04 \\
\hline Enterococcus faecium & 0.50 & 1.48 & 0.90 & 0.58 \\
\hline unclassified Enterococcus & 0.58 & 2.08 & 1.11 & 0.73 \\
\hline Faecalibacterium prausnitzii & 0.40 & & 1.20 & \\
\hline unclassified Bacteroides & 1.12 & & 3.91 & 0.59 \\
\hline unclassified Faecalibacterium & 0.33 & & 0.84 & \\
\hline Bacteroides vulgatus & 0.07 & & & \\
\hline Bacteroides caccae & 44.35 & & & \\
\hline unclassified Eubacterium & 0.51 & & 6.39 & \\
\hline unclassified Lactococcus & 0.00 & & & \\
\hline Bacteroides thetaiotaomicron & 6.80 & & & \\
\hline Bifidobacterium pseudocatenulatum & & 0.03 & 0.19 & \\
\hline Lactobacillus gasseri & & 11.16 & & \\
\hline Enterococcus faecalis & & & 5.78 & \\
\hline Lactococcus lactis & & & 0.94 & \\
\hline Enterococcus gallinarum & & & 0.81 & \\
\hline
\end{tabular}

Table S4: Bifidobacterium species abundance from the fermentation of inulin.

\begin{tabular}{|l|c|c|}
\hline \multirow{2}{*}{ Species } & \multicolumn{2}{c|}{ Abundance (\% from total reads) } \\
\cline { 2 - 3 } & L-Inulin-t0 & L-Inulin-t72 \\
\hline Bifidobacterium adolescentis & 5.3 & 3.6 \\
\hline Bifidobacterium animalis & 0.3 & 0 \\
\hline Bifidobacterium pseudocatenulatum & 0.2 & 0 \\
\hline \multirow{2}{*}{ Species } & \multicolumn{2}{|c|}{ Abundance (\% from total reads) } \\
\cline { 2 - 3 } & 0-Inulin-t0 & 0-Inulin-t72 \\
\hline Bifidobacterium adolescentis & 0.4 & 24.8 \\
\hline Bifidobacterium longum & 4.6 & 0.2 \\
\hline Bifidobacterium pseudocatenulatum & 0.2 & 1 \\
\hline Bifidobacterium bifidum & 0 & 0.1 \\
\hline
\end{tabular}


In both cases (L and O-fermentations) B. adolescentis dominated the bifidobacterial species after $72 \mathrm{~h}$. In lean microbiota a complete domination was observed while in the obese microbiota it was about 25 fold higher (whereas at the start it was only 8\%). Previous testing of the in vitro effects of IN fermentation in both microbiotas (lean and obese) found that the obese fermentation of IN produced higher concentrations of $n$ butyrate when compared to the lean fermentation $[16,18]$. The difference with our study could be explained by the different stimulation of certain butyrogenic strains, which in this particular case found more favourable to growth in the lean microbiota as observed in Tables S3 and S4.

Figure S1: Abundance (\%) of the major genera in the microbiota from lean and obese subjects.

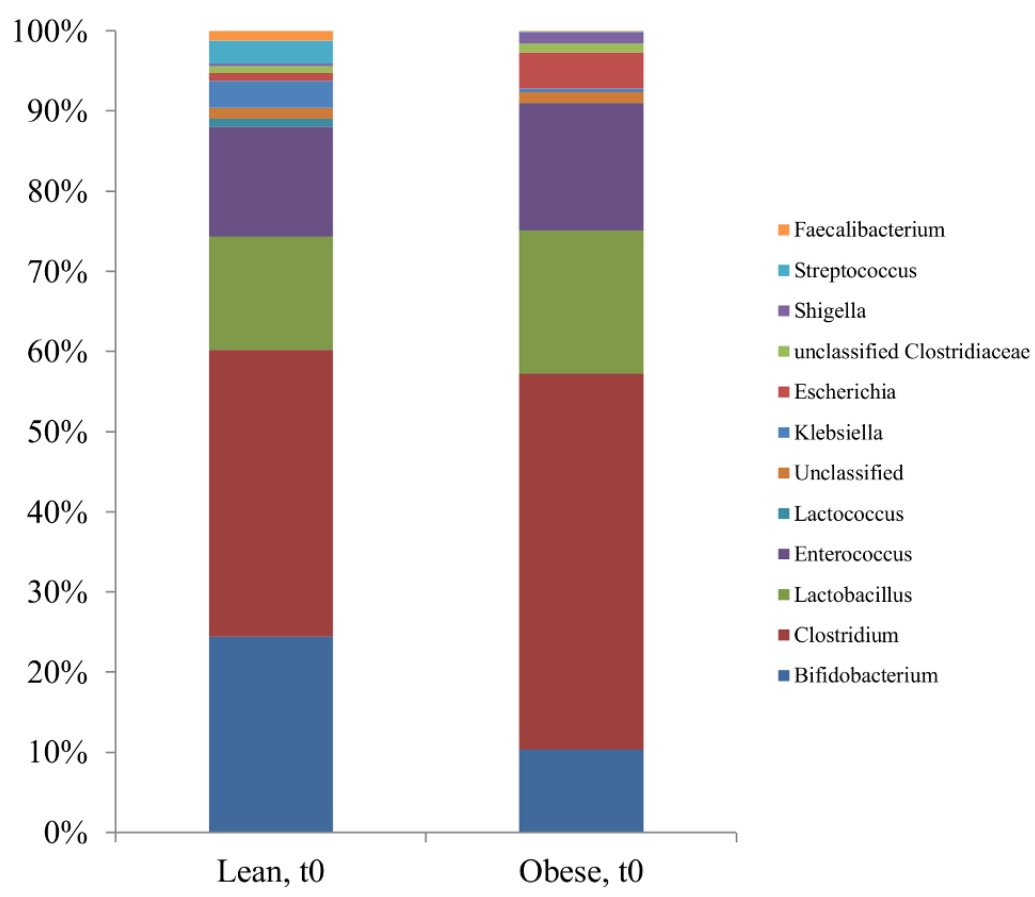

At genus level, it was observed that differences among the inocula were driven by the abundances within the same groups of bacteria (Figure S1). The major genera in the lean microbiota were Bifidobacterium, Clostridium, Lactobacillus and Enterococcus $(23,34,14$ and $13 \%$, respectively) and in the obese microbiota were Clostridium, Lactobacillus, Enterococcus, Bifidobacterium (46, 17, 15 and 10\%). Analysis of the species found in the inoculum (t0) suggests that the lean microbiota had a more diverse population of Bifidobacterium that included B. adolescentis, B. longum and an unclassified group (7, 4 and $8 \%$, respectively) whilst the obese microbiota mainly contained $B$. longum and an unclassified group ( 6 and 3\%, respectively). Both microbiotas shared a high abundance of $C$. butyricum (lean: $23 \%$; obese: $30 \%$ ). 


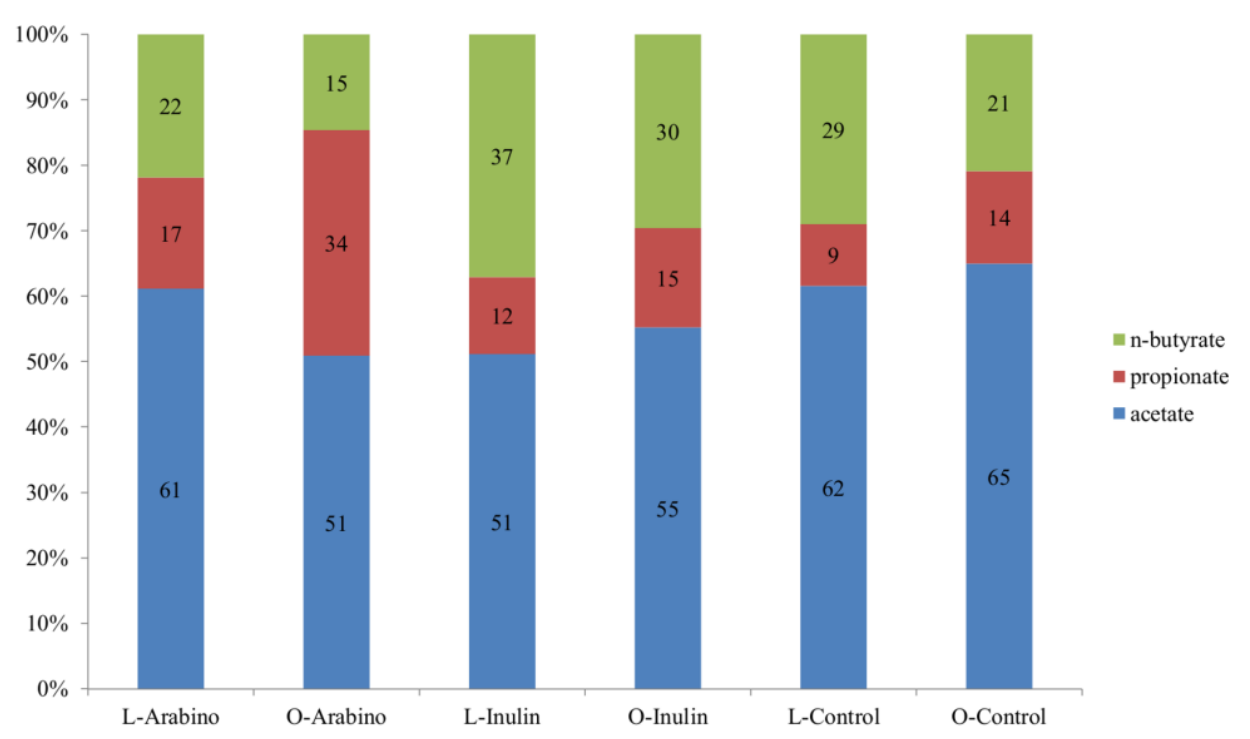

Figure S2: SCFA ratios (\%) from the different diets at $\mathrm{t} 72$. 


\section{Chapter 7}

\section{Potential of pectins to beneficially modulate the gut microbiota depends on their structural properties}

Submitted:

Larsen N., Bussolo de Souza C., Cahú T. B., Krych L., Wiese M., Kot W., Hansen K. M., Blennow A., Venema K., Jespersen L. Potential of pectins to beneficially modulate the gut microbiota depends on their structural 


\begin{abstract}
Pectins are plant cell-wall polysaccharides which can be utilized by commensal bacteria in the gut, exhibiting beneficial properties for the host. Knowledge of the impact of pectins on intestinal bacterial communities is insufficient and limited to a few types of pectins. This study characterized the relationship between the structural properties of pectins and their potential to modify composition and activity of the gut microbiota in a beneficial way. For this purpose, we performed in vitro fermentation of structurally diverse pectins from citrus fruits and sugar beet (nine in total) and a pectic derivative, rhamnogalacturonan I (RGI), using a TIM-2 colon model. The composition of the microbiota throughout TIM-2 fermentations was assessed by $16 \mathrm{~S}$ rRNA gene amplicon sequencing. Both general and pectin-specific changes were observed in relative abundances of numerous bacterial taxa in a time-dependent way. Bacterial populations associated with human health, such as Faecalibacterium prausnitzii, Coprococcus, Ruminococcus, Dorea, Blautia, Oscillospira, Sutterella, Bifidobacterium, Christensenellaceae, Prevotella copri, and Bacteroides spp. were either increased or decreased depending on the substrate, suggesting that these bacteria can be controlled using structurally different pectins. The main structural features linked to the pectin-mediated shifts included degree of esterification, composition of neutral sugars, distribution of homogalacturonan and rhamnogalacturonan fractions, degree of branching and the presence of amide groups. Cumulative production of total short chain fatty acids and propionate was largest in fermentation of sugar beet pectin $(162.6 \mathrm{mmol}$ and $43.3 \mathrm{mmol}$, respectively) and RGI (159.4 $\mathrm{mmol}$ and $47.6 \mathrm{mmol}$, respectively). This study provides evidence that the human gut microbiota can be modulated by pectins and identifies specific features linked to microbial alterations. This knowledge can be used to define preferred dietary pectins, targeting beneficial bacteria and favoring more balanced microbiota profiles in the gut.
\end{abstract}




\section{Introduction}

Pectins are part of a daily diet consumed in the form of fruits and vegetables. Besides, they are authorized as food additives and used as emulsifiers, gelling or stabilizing agents in yoghurts, jams and other food products (1). Pectins are commercially produced from peel and pulp of fruits and vegetables, mainly citrus fruits, sugar beet and apples. Structural differences of pectins are defined by their source and production methods. The backbone of pectin molecules is composed of homogalacturonan, or 1,4-linked $\alpha$-D-galacturonic acid, occasionally substituted by rhamnose. The major side chain unit is rhamnogalacturonan I (RGI) ("hairy" region), consisting of $\alpha-(1,4)$ galacturonosyl and $\alpha$-(1,2)-rhamnosyl chains with attached neutral sugars (galactan, arabinan or arabinogalactans) (2). The polygalacturonic acid in the backbone can be partially esterified with methyl groups. According to the degree of esterification (DE), pectins are conventionally referred to as high methoxyl (HM) pectins (DE > 50\%) and low methoxyl (LM) pectins (DE < 50\%). Commercial LM pectins can also be amidated in order to achieve better gelling control (3).

Pectins are indigestible by human enzymes, however, they can be easily degraded by commensal bacteria in the gut with concomitant production of short chain fatty acids (SCFA) and other metabolites (4). Besides SCFA production, the beneficial effects of pectins include reduction of ammonia (5), delayed gastric emptying and improved glucose metabolism (6). Furthermore, pectins can induce gut immunity, improve intestinal integrity and mucosal proliferation, and favor adhesion of probiotic Lactobacillus strains to epithelial cells $(4,7,8)$. The ability of pectins and pectic oligosaccharides (POS) to support the growth of specific bacterial populations has been described in several studies; however, there is some inconsistency in the results. Pectins and POS were found to stimulate beneficial bacteria, including, bifidobacteria, lactobacilli, Faecalibacterium, Roseburia and Eubacterium rectale (5,9-11), whereas other in vitro studies reported unchanged or even decreased levels of bifidobacteria and Roseburia (12-14). Other bacterial taxa commonly increased in pectin fermentations, include Bacteroides, Prevotella, E. rectale/C. coccoides group and Clostridium spp. (12-17). Species within the genera Bacteroides and Prevotella are considered to be the primary pectin-degraders, as they possess carbohydrate-active enzymes (CAZymes) encoded by the polysaccharide utilization loci (PULs) (18). The CAZymes comprise lyases, methylesterases and acetylesterases, facilitating the breakdown of pectins (19). Variations in microbiota composition, enzyme capabilities and fermentation substrates, can 
explain inconsistencies between the studies in the effects of pectins and POS on microbial communities.

Functional properties of pectins in the gut are influenced by their structural features, e.g., distribution of free and methylated carboxyl groups within the polygalacturonic acid, molecular size and sugar composition $(8,13,20)$. Vogt et al. (21) demonstrated that immune activation of T84 cells and protection of intestinal barrier function upon exposure to lemon pectins was related to the degree of esterification (DE). Fermentations with human feces and mixed cultures showed that pectins with lower DE and lower oligomeric size were preferentially metabolized and stimulated the growth of bifidobacteria (22-24). In a recent study, Tian and coworkers reported differences between LM and HM pectins on the levels of fecal Prevotella and Lactobacillus in piglets (25). Different abundances of Bifidobacterium, Bacteroides/Prevotella and Clostridium coccoides/Eubacterium rectale group were found in fermentations of structurally different pectins from lemon and sugar beet (10).

In previous studies we observed that structural factors, such as DE, net charge, degree of branching and molecular weight, were related to the ability of pectins to improve survival of probiotic strains under simulated gastro-intestinal conditions (8). Up to now, the impact on the gut microbiota has been studied for just a few types of pectins, and the significance of their structural properties for microbiota shaping remains unclear. To clarify these issues, we performed fermentations of structurally diverse pectins from citrus fruits and sugar beet using the TIM-2 colon model and characterized their potential to modify the gut bacterial populations in a beneficial way in relation to their structural features.

\section{Materials and methods}

\section{Pectins}

Pectins for this study (nine in total) were produced by CP Kelco (Denmark) from orange (P1 and P8), lemon (P2, P3, P6 and P9), lime (P5 and P7) and sugar beet (P4) using different extraction methods (harsh, mild and differentially extracted) as shown in Table 1. Rhamnogalacturonan I (P10) was purified from lime. Additionally, pectins P5 and P6 were chemically and enzymatically de-esterified, respectively, and pectins $\mathrm{P} 7$ and $\mathrm{P} 8$ were amidated according to the standard procedure at CP Kelco. 
Table 1: Pectins used in this study

\begin{tabular}{lll}
$\begin{array}{l}\text { Pectin } \\
\text { ID }\end{array}$ & Production methods & Source \\
\hline P1 & Harsh extracted & Orange \\
P2 & Mild extracted & Lemon \\
P3 & Differentially extracted & Lemon \\
P4 & Harsh extracted & Sugar beet \\
P5 & Harsh extracted chemically de-esterified & Lime \\
P6 & Mild extracted enzymatically de-esterified & Lemon \\
P7 & Harsh extracted amidated & Lime \\
P8 & Harsh extracted amidated & Orange \\
P9 & Harsh extracted & Lemon \\
P10 & Rhamnogalacturonan I & Lime \\
\hline
\end{tabular}

\section{High Performance Anion Exchange Chromatography (HPAEC)}

Monosaccharide composition of pectins was determined by high performance anion exchange chromatography (HPAEC) equipped with a PA20 column and Pulsed Amperometric Detection (PAD, Dionex, CA, USA) as described previously (8). Monosaccharides, galacturonic acid (GalA), arabinose (Ara), rhamnose (Rha), galactose (Gal), glucose (Glc), and xylose (Xyl) were quantified from two independent HPAEC-PAD analyses. Degree of branching (DBr) of the rhamnosyl residues with neutral sugar side chains was calculated by the equation $\operatorname{DBr}(\%)$ $=100 \% \times \mathrm{Rha}(\%) /[(\mathrm{Ara}(\%)+\mathrm{Gal}(\%)]$. The molar content of homogalacturonan $(\mathrm{HG})$ and rhamnogalacturonan $(\mathrm{RG})$ was estimated using equations: $\mathrm{HG}(\%)=$ GalA(\%) - Rha(\%); RG(\%) = 2Rha(\%) + Ara(\%) + Gal(\%) (26). Differences in the composition of monosaccharides between the pectins were tested by one-way ANOVA, with a Tukey's post-hoc test (Supplementary Table S1).

\section{TIM-2 fermentations and sample collection}

In vitro fermentations were performed using the TIM-2 proximal colon model (TNO Innovation for Life, the Netherlands), validated and used as a predictive model for clinical trials (14). The TIM-2 model provides fully anaerobic conditions, peristaltic movements and removal of metabolites during fermentation. The microbiota inoculum consisted of active, pooled fecal samples from 8 healthy adults (male: $n=4$, female: $n=4$; age of $25-42$ years). Fecal samples were maintained under anaerobiosis by using anaerobic packs (AnaeroGen ${ }^{\mathrm{TM}}$, Oxoid, Cambridge, UK). The fecal microbiota was homogenized under anaerobic conditions, snap-frozen in liquid nitrogen and stored at $-80{ }^{\circ} \mathrm{C}$ 
before inoculation in TIM-2. The TIM-2 colon model was operated as follows. All units of the system were flushed with nitrogen prior to inoculation and throughout the experiments. Fermentations were performed at $37^{\circ} \mathrm{C}$ with the $\mathrm{pH}$ kept at 5.8 (or slightly above) by automatic titration with $2 \mathrm{M} \mathrm{NaOH}$. A $30 \mathrm{ml}$ portion of microbiota homogenate was used to inoculate the units for each experiment. Following inoculation, the microbiota was incubated for $16 \mathrm{~h}$ for adaptation to the new environment. During this period the basal simulated ileal efflux medium (SIEM), composed of complex carbohydrates, protein, ox-bile, Tween 80 , vitamins and minerals (27), was gradually introduced into the system in a total volume of $40 \mathrm{ml}$; afterwards, the culture was deprived from any medium for $2 \mathrm{~h}$ (starvation period). The units were then fed with SIEM containing $7.5 \mathrm{~g}$ pectin per day as the only carbohydrate source. The doses of pectin in TIM-2 fermentations were based on the amount of carbohydrates present in SIEM, and corresponded to approximately $250 \mathrm{~g}$ citrus fruit (grapefruit, lemon, orange) with the typical content of pectins of $2.3-4.5 \%$ (28). Lumen and dialysate samples were taken at the start of fermentation $(0 \mathrm{~h})$ and after 24, 48, 56 and $72 \mathrm{~h}$ fermentation for analyses of microbiota, SCFA and branched chain fatty acids (BCFA). Samples were snap-frozen in liquid nitrogen and stored $\left(-80^{\circ} \mathrm{C}\right)$ until analysis. Fermentation of each substrate was performed in two independent TIM-2 experiments.

\section{DNA purification}

Microbiota samples from TIM-2 experiments were centrifuged $(10.000 \times \mathrm{g}, 10$ $\mathrm{min}$ ) and the fecal water discarded. The total bacterial DNA was isolated from the pellet using the PowerLyzer@PowerSoil DNA Isolation Kit (Qiagen Nordic, Denmark) according to manufacturer's protocol with few modifications. Modifications included resuspension of fecal slurries in the Bead Solution, transfer to the PowerLyser $\AA$ Glass bead tubes and heating the tubes at $65^{\circ} \mathrm{C}$ for $10 \mathrm{~min}$ before the bead beating step. The homogenization was performed at speed setting of $6.5 \mathrm{~m} / \mathrm{sec}$ for 3 cycles, $30 \mathrm{sec}$ each with $30 \mathrm{sec}$ intervals (FastPrep ${ }^{\circledR}-24$, MP Biomedicals, Solon OH, USA). The concentration and purity of DNA was determined using the NanoDrop $^{\mathrm{TM}} 2000$ Spectrophotometer (Thermofisher Scientific, Denmark). The DNA concentration was typically in the range of $20-100 \mathrm{ng} / \mu \mathrm{l}$ and A260/A280 ratios of $1.8-2.1$.

\section{$16 S$ rRNA gene amplicon sequencing and data processing}

The DNA library for amplicon sequencing was prepared according to Williams et al. (29). In brief, the V3 region ( $190 \mathrm{bp}$ ) of the $16 \mathrm{~S}$ rRNA gene was PCR amplified using AccuPrime SuperMix II (Life Technologies, CA, USA) and primers NXt_388F 
and NXt_518R compatible with Nextera Index Kit (Illumina). Amplification steps included initial denaturation at $95^{\circ} \mathrm{C}$ for $2 \mathrm{~min}, 33$ cycles of denaturation at 95 ${ }^{\circ} \mathrm{C}$ for $15 \mathrm{~s}$, annealing of primer at $55^{\circ} \mathrm{C}$ for $15 \mathrm{~s}$ and elongation at $68^{\circ} \mathrm{C}$ for $30 \mathrm{~s}$. The second PCR (PCR II) was performed using Phusion High-Fidelity PCR Master Mix (ThermoFisher Scientific, USA, MA) and primers P5 and P7 (Nextera Index Kit) to incorporate adapters and tags in the PCR product. The PCR II setup included 13 cycles of denaturation at $98^{\circ} \mathrm{C}$ for $10 \mathrm{~s}$, annealing of primer at $55^{\circ} \mathrm{C}$ for $20 \mathrm{~s}$ and elongation at $72{ }^{\circ} \mathrm{C}$ for $20 \mathrm{~s}$, followed by an extension at $72{ }^{\circ} \mathrm{C}$ for 5 min. Amplifications were performed in a Veriti ${ }^{\circledR}$ 96-well thermal cycler (Applied Biosystems, USA). The amplified fragments with adapters and tags were purified using AMPure XP beats (Beckman Coulter Genomic, CA, USA). Tag-encoded 16S rRNA gene sequencing was performed using Illumina NextSeq platform using the 2x150 cycles MID V2 kit (Illumina, CA, USA). The raw dataset, containing pairended reads with corresponding quality scores, were merged and trimmed, using fastq_mergepairs and fastq_filter scripts implemented in the UPARSE pipeline, as reported previously (29).

\section{Analysis of sequencing data and statistic}

Quantitative Insight Into Microbial Ecology (QIIME) software package (version 1.8.0) was used for subsequent analysis steps (30). The sequences were classified using Greengenes database (version 13.8) as a reference 16S rRNA gene database (31). Alpha-diversity was evaluated by the number of observed species and Chao 1 computed for each OTU table rarefied to 10.000 sequences per sample (lowest number of sequences produced per sample). Good's coverage was estimated to assess the depth of sequencing. Differences in alpha-diversity between times were tested using Student's t-test, employing the non-parametric (Monte Carlo) method (999 permutations). Differences in Chao 1 indices between the pectins after 24, 48 and $72 \mathrm{~h}$ fermentation were assessed by a oneway analysis of variance (ANOVA) with LSD post-hoc test. Community differences (beta-diversity) were revealed by weighted and unweighted UniFrac distance matrices computed from the rarefied OTU tables and evaluated by analysis of similarities (ANOSIM). Principal Coordinate Analysis (PCoA) plots were generated with Jackknifed Beta Diversity workflow using 10 subsampled OTU tables. Group differences in microbial community structure were assessed by hierarchical clustering of OTUs at species level, applying UPGMA (unweighted pair group method using arithmetic averages) algorithm. Differences between the pectins were assessed in the pooled data set (48, 56 and $72 \mathrm{~h}$ from two independent experiments) compared to time $0 \mathrm{~h}$ (baseline) by the nonparametric Wilcoxon Rank Sum test combined with Bonferroni multiplicity 
correction, using 0.05 as significance level. Correlation between the relative abundances of OTUs and the structural characteristics of pectins (rhamnogalacturonan RGI was not included) was evaluated with the Spearman rank correlation test implemented in the otu_category_significance.py script (QIIME 1.8.0). Statistical significance was evaluated from the conservative false discovery rate (FDR) corrected p-values for multiple comparisons (FDRadjusted, $\mathrm{p}<0.05$ ).

\section{Analysis of short chain and branched chain fatty acids}

Analyses of SCFA and BCFA were performed in the lumen and dialysate of TIM-2 samples by Brightlabs (Venlo, the Netherlands). Samples were centrifuged $(10.000 \times \mathrm{g}, 20 \mathrm{~min})$ and the supernatants were diluted with $1.5 \mathrm{mM}$ sulfuric acid. The samples $(10 \mu \mathrm{l})$ were injected onto an 883 Basic IC plus ion-chromatography system with suppressed conductivity detection used in positive mode (Metrohm, Herisa, Switzerland). The acids were separated by isocratic elution on ICsep ION300 Ion exclusion column (300 $\mathrm{mm} \times 7.8 \mathrm{~mm}, 7 \mu \mathrm{m}$ particle size) and Metrosep RP2 Guard column (Transgenomic, New Haven, CT, USA) using $1.5 \mathrm{mM}$ sulfuric acid as a mobile phase. Samples were eluted at a flow rate of $0.4 \mathrm{ml} / \mathrm{min}$ with a pressure of $5.5 \mathrm{MPa}$ at $65^{\circ} \mathrm{C}$. Acetic, propionic, butyric, iso-butyric and isovaleric acids were used as calibration standards (Sigma Chemical, St. Louis, MO). Statistical differences between the pectins in SCFA and BCFA production over time were assessed by one-way ANOVA, with Tukey's post-hoc test. Differences between the HM pectins (P1, P2, P3 and P4) and LM pectins (P5, P6, P7, P8 and P9) were evaluated by the two-sided Wilcoxon Rank Sum test. Correlation between the relative abundances of OTUs and cumulative production of fatty acids was assessed with the Spearman rank correlation test (QIIME 1.8.0) and statistical significance was evaluated from the FDR corrected $p$-values. In all tests differences were assumed to be statistically significant at $\mathrm{p}$-values $<0.05$.

\section{Results}

\section{Composition and structural properties of pectins}

In total nine pectins (P1 - P9) and rhamnogalacturonan I (P10), were used as substrates for microbiota fermentation in the TIM-2 colon model (Table 1). The composition and physicochemical properties of the pectins have been described previously (8). The structural parameters referred to in this study, including monosaccharide composition, $\mathrm{DE}$ and $\mathrm{DBr}$, are presented in Supplementary Table S1. Pectins P1, P2, P3 and P4 were HM pectins with highest DE of 70.0 and 74.7 \% in P2 and P3, respectively; others were LM pectins with DE between 11.4 
- $35.6 \%$, lowest in P5. The major neutral sugars in pectin molecules were galactose (Gal, 9.1 - $30.7 \%$ ), arabinose (Ara, 0.8 - 16.7\%), rhamnose (Rha, 1.7 $3.7 \%$ ) and glucose (Glc, $0.7-8.7 \%$ ), while xylose (Xyl) was found in lesser amounts $(<1 \%)$. Content of galacturonic acid (GalA) and homogalacturonan (HG) was largest in pectins P2, P3, P6 and P9 from lemon (GalA of $70.7-83.0 \%$ and HG of $69.6-81.2 \%$ ) and lowest in sugar beet pectin (P4) (46.9\% of GalA and $43.9 \%$ of $\mathrm{HG}$ ). Additionally, sugar beet pectin was characterized by relatively higher fractions of RG (50.7\%) and neutral sugars (Gal, Ara and Rha). Rhamnogalacturonan I (P10) differed from pectins by low percentage of GalA $(11.0 \%)$ and galactose (7.9\%), and high fraction of arabinose (69.9\%), rhamnose (6.6 \%) and xylose (1.8\%). Estimated degree of branching (DBr) of rhamnosyl residues with neutral sugars side chains varied between $7.4 \%$ (P3) and $19.2 \%$ (P6).

\section{Alpha-diversity of microbiota}

Alpha-diversity of microbiota was affected by the fermentation time and substrates, as presented by Chao 1 indices (Figures 1A,B) and observed species number (Supplementary Figures S1 and S2). The Chao 1 values were significantly increased from $723 \pm 22$ at baseline to $746 \pm 30$ after $24 \mathrm{~h}$ followed by gradual decrease to $640 \pm 27$ after $72 \mathrm{~h}$ fermentation (Figure $1 \mathrm{~A}$ ).
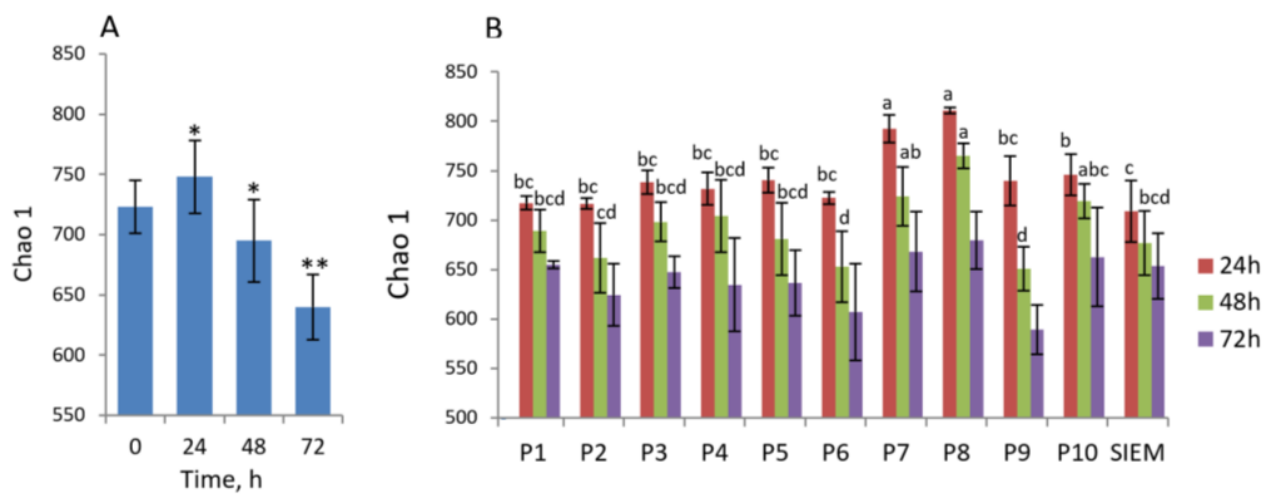

Figure 1: Analysis of alpha-diversity over fermentation time and between the pectins presented by Chao1 index. (A) Chao1 at baseline $(0 \mathrm{~h})$ and after 24, 48 and $72 \mathrm{~h}$ fermentation computed for combined samples ( $\mathrm{n}=20$ for each time point). Asterisks denote the values significantly different from time $0 \mathrm{~h}$ (non-parametric Student's t-test, ${ }^{*} \mathrm{p}<0.05$, ${ }^{* *} \mathrm{p}<0.01$ ). (B) Chao1 after $24 \mathrm{~h}$ (brown columns), $48 \mathrm{~h}$ (green columns) and $72 \mathrm{~h}$ (purple columns) fermentation of pectins P1 - P10 and SIEM, presented by means and standard deviation (bars) from two biological repeats. Superscripts (a, b, c, and d) show significant differences between the substrates at time-points 24 and $48 \mathrm{~h}$ (one-way ANOVA, LSD post-hoc t test, $\mathrm{p}<0.05$ ). Chao1 indices at $72 \mathrm{~h}$ were not significantly different (letters not shown). 
Highest diversity was observed for pectins P7 and P8 (Chao 1 of $724 \pm 30$ and $765 \pm 12$ after $48 \mathrm{~h}$, respectively) and lowest for P6 and P9 fermentations (Chao 1 of $653 \pm 36$ and $651 \pm 22$ after $48 \mathrm{~h}$, respectively) (Figure 1B). Differences in alpha-diversity after $72 \mathrm{~h}$ fermentation were insignificant. Though the rarefaction curves did not completely reach a plateau (Supplementary Figure S2), the coverage estimates (Good's coverage) were over $98.2 \%$ across the samples (data not shown), suggesting that sampling depth of 10.000 reads was sufficient to capture the majority of OTUs.

\section{Group diversity over fermentation time}

Analysis of group diversity (beta-diversity) across the sampling times (Figures $2 \mathrm{~A}, \mathrm{~B}$ ) and the pectins (Figures $3 \mathrm{~A}, \mathrm{~B}$ ) is presented by the PCoA plots using unweighted and weighted UniFrac distance matrices.

Unweighted UniFrac matrix is based on the presence/absence of bacterial species, accounting for both abundant and rare lineages; while the weighted matrix relies also on the absolute proportions, and it is most sensitive to detect the changes in dominant taxa. Major shifts in microbial diversity occurred after $24 \mathrm{~h}$ fermentation, as seen from both weighted and unweighted PCoA and confirmed by ANOSIM ( $p=0.001$ ) (Figure $2 A, B)$. Differences in group diversity between the time-points 48,56 and $72 \mathrm{~h}$ were insignificant, especially for weighted UniFrac $(R=0.002, p=0.774$; Figure $2 B)$, indicating similarity in microbial communities. Though a pattern of pectin clustering was similar for both types of analyses (Figure 3A,B), variation between the groups could be best explained by the weighted PCoA (PC1 $66 \%$ and PC2 $27 \%$ ), suggesting the impact of abundant bacterial taxa for group differentiation. Pectins P4 and P10 (group 1) were distinctly separated from other substrates along the PC1 (weighted PCoA, Figure 3B); other clusters combined pectins P1, P2 and P3 (group 2), pectins P7 and P8 (group 3) and pectins P6 and P9 (group 4, unweighted PCoA, Figure 3A). 


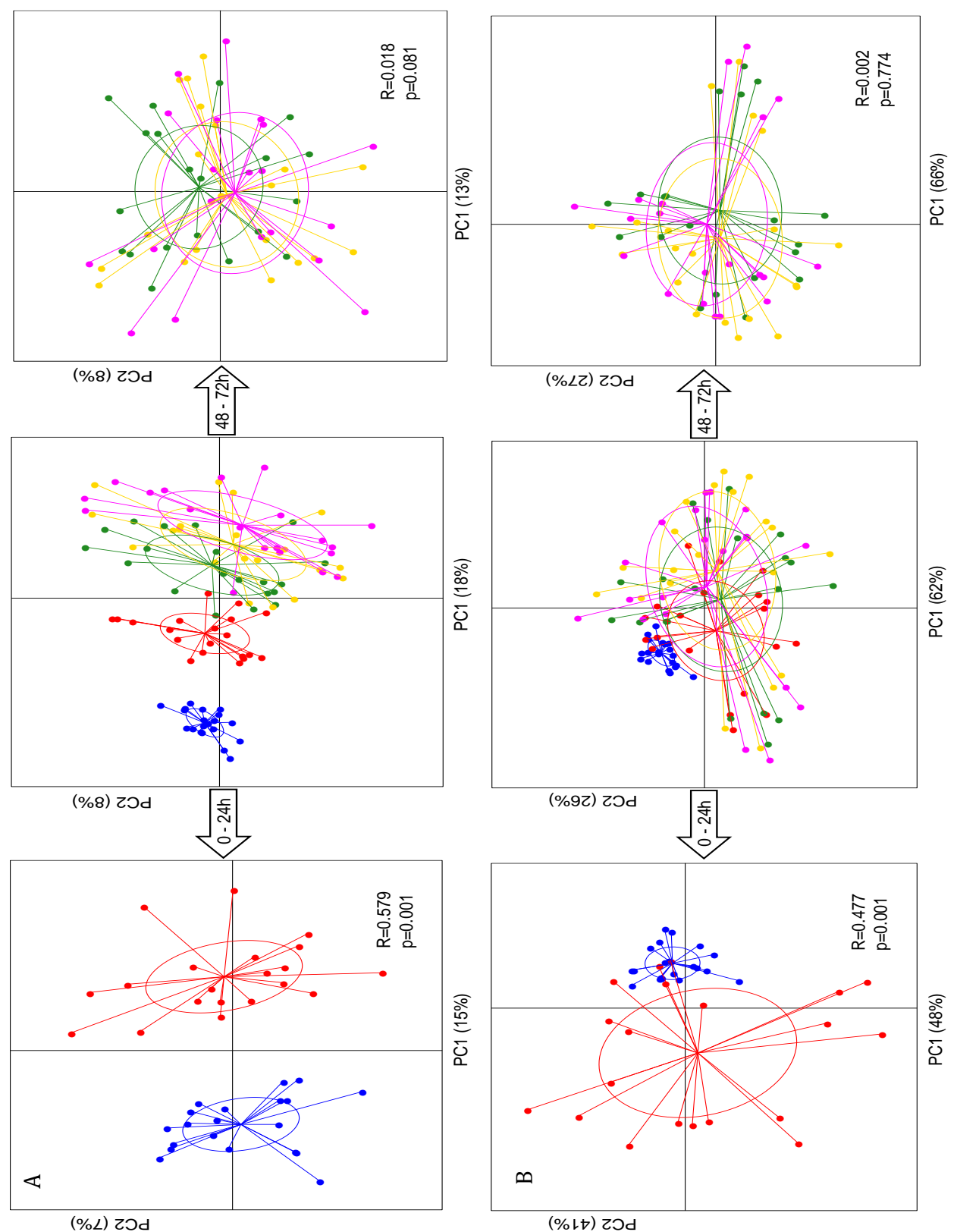

Figure 2. Principal Coordinate Analysis (PCoA) plots, showing group diversity over time, $0-72 \mathrm{~h}$ (middle plots), 0 - $24 \mathrm{~h}$ (plots to the left), and $48-72 \mathrm{~h}$ (plots to the right), assessed by unweighted (A) and weighted (B) UniFrac distance matrices. Samples are colored according to the time-points: blue dots $-0 \mathrm{~h}$, red dots $-24 \mathrm{~h}$, green dots $-48 \mathrm{~h}$, yellow dots - $56 \mathrm{~h}$, and pink dots $-72 \mathrm{~h}$. Samples were collected from two independent fermentations of each substrate (P1 - P10). Group differences were tested by ANOSIM as shown by R-values and p-values. The samples are plotted on the first two principal coordinates PC1 and PC2. The ellipse center indicates group means for each time-point. 

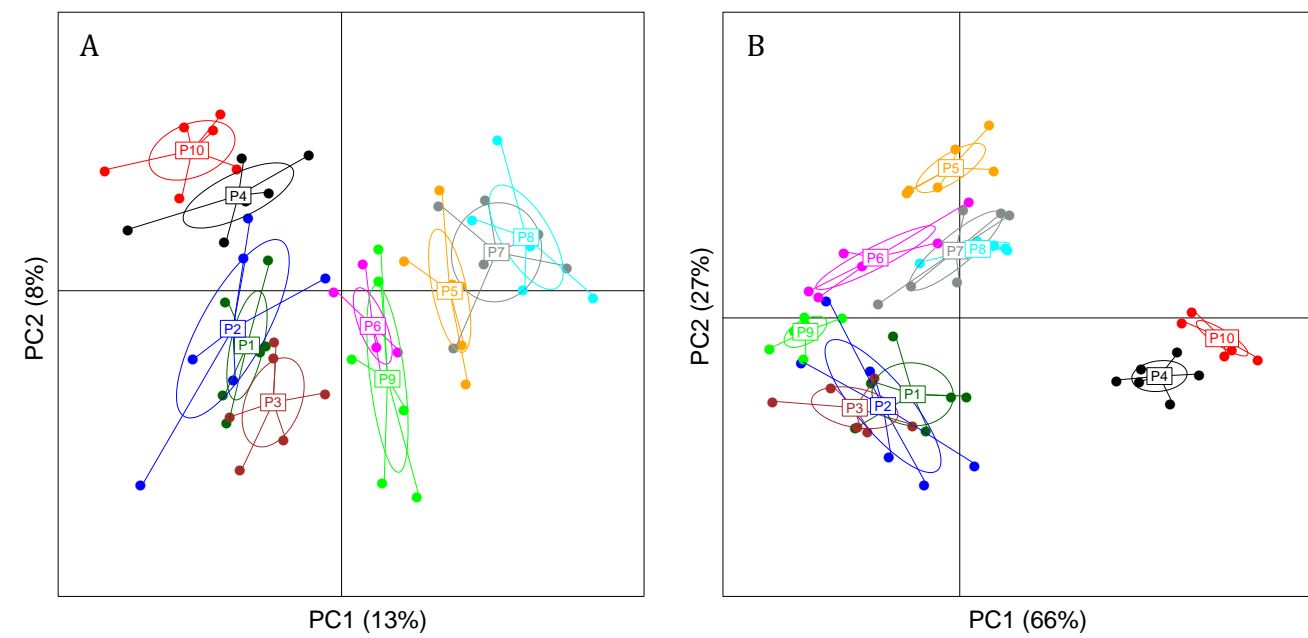

Figure 3: Principal Coordinate Analysis (PCoA), showing group diversity between the pectins, as determined by unweighted (A) and weighted (B) UniFrac distance matrices. PCoA plots were constructed for the samples collected after 48, 56 and $72 \mathrm{~h}$ fermentation from two independent experiments ( $\mathrm{n}=6$ for each pectin). The ellipse center indicates group means (P1 - P10). The samples are plotted on the first two principal coordinates PC1 and PC2.

\section{Changes in relative abundances of bacterial species during fermentation}

In total 1218 species-level bacterial OTUs were assigned to more than 150 bacterial taxa. Figure 4 shows the relative abundances of species-level OTUs at baseline $(0 \mathrm{~h})$ and after 48, 56 and $72 \mathrm{~h}$ fermentation (cut-off $0.01 \%$ ) and the hierarchical clustering of pectins. Regarding high similarity between the samples collected after $48 \mathrm{~h}$ (Figure 2A,B), statistical differences between the pectins were evaluated in the pooled dataset (combined 48,56 and $72 \mathrm{~h}$ samples). 


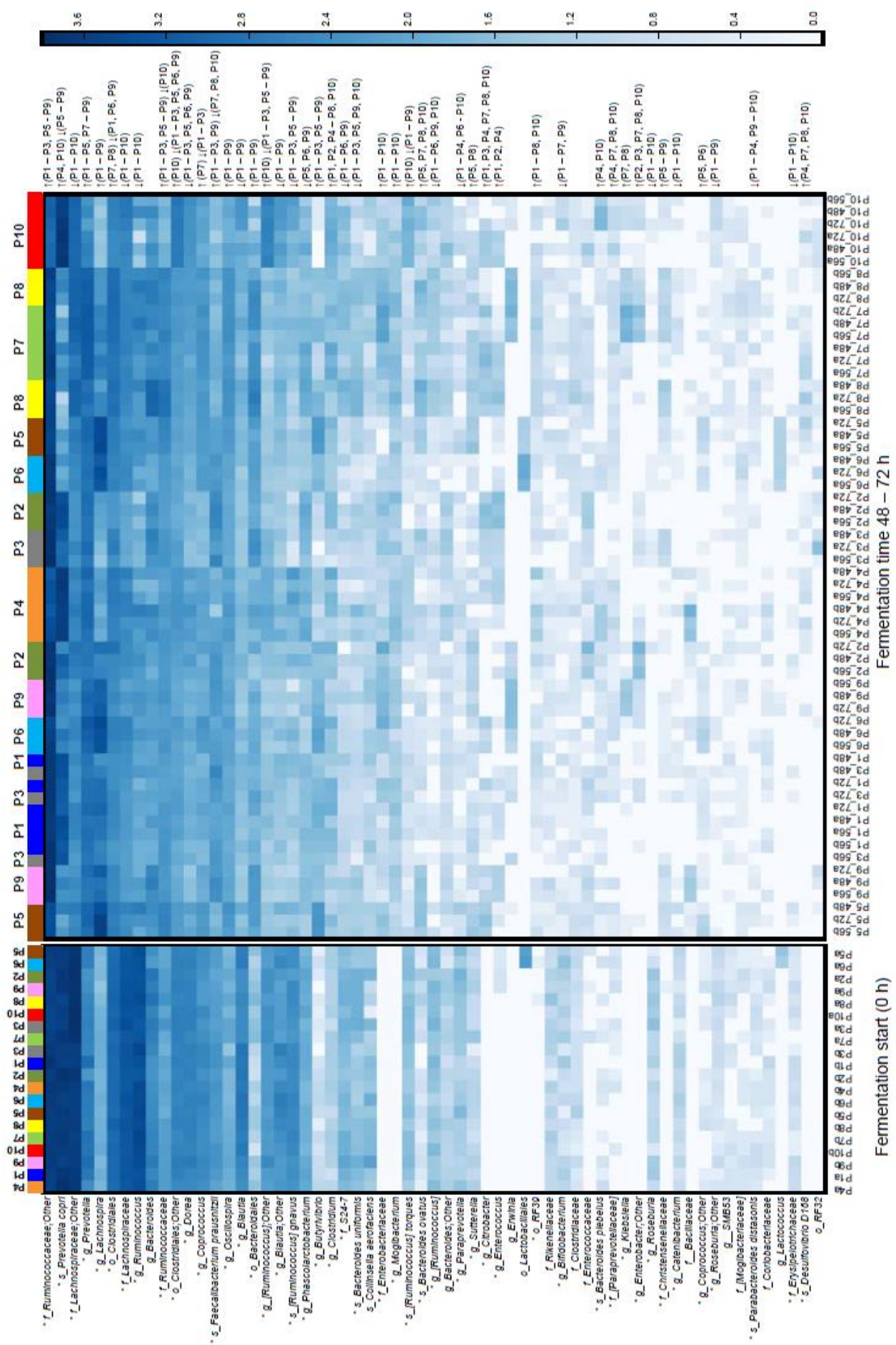


Figure 4: Heatmap showing distribution of bacterial taxa across the pectins at start $(0 \mathrm{~h})$ and after 48 - $72 \mathrm{~h}$ fermentation, based on hierarchical clustering (UPGMA). Each row presents relative abundances of OTUs assigned at species level (cut-off 0.01\%). Color scale indicates relative abundance in percentage. A color panel above the heatmap shows clustering of pectins labelled by different colors. Arrows and pectins (in brackets) to the right of the heatmap indicate significant increases or decreases in bacterial populations (marked by asterisks). Differences were analyzed for combined samples (48, 56 and 72 $h, n=6$ for each pectin) in two independent experiments ( $a$ and $b$ ), using the Wilcoxon Rank Sum test and Bonferroni multiplicity correction ( $p<0.05)$. Taxa denoted as "Other" indicates ambiguity in the assignment meaning that more than one taxon could be assigned to this cluster at given taxonomic level. Taxa in square brackets indicate a proposed taxonomy.

Four bacterial taxa, i.e., family Lachnospiraceae (19.3 \%), family Ruminococcaceae (16.5\%) and genus Ruminococcus (10.4\%) within phylum Firmicutes, and species Prevotella copri (18.3\%) within phylum Bacteroidetes were prevalent at the start of the fermentation (64.5\% in total). Twenty-six other bacterial populations, belonging to phyla Firmicutes and Bacteroidetes, were found in moderate abundance (0.1 - $10 \%)$; amongst which Blautia, Dorea, Coprococcus, Ruminococcus, Faecalibacterium prausnitzii, Bacteroides and Prevotella were most abundant. Less abundant phyla at baseline were Actinobacteria (0.41\%), presented mainly by Bifidobacterium and Collinsella, phylum Proteobacteria (0.09\%), presented by Desulfovibrio spp., Sutterella and Enterobacteriaceae, and phylum Tenericutes (0.02\%), comprising Anaeroplasma and order RF39. Fermentation of pectins was accompanied by both general and pectin-specific shifts in bacterial abundances. Bacterial populations reduced by all substrates, though to a different extent, included Ruminococcus spp., genera Blautia, Roseburia, Catenibacterium, Bifidobacterium, Paraprevotella, and unassigned Lachnospiraceae and Erysipelotrichaceae. Concurrently, proportions of Lachnospira, Oscillospira, Clostridium, Butyrivibrio, Prevotella, Bacteroides spp., unclassified family Ruminococcaceae and order Bacteroidales, were increased. Additionally, the rare taxa within Mogibacterium (phylum Firmicutes), unassigned family Enterobacteriaceae (phylum Proteobacteria) and order RF39 (phylum Tenericutes) were highly stimulated in all fermentations. Pectin-specific bacterial shifts which could partially explain grouping of P4 and P10 (Figure 3B), included enrichment in Prevotella copri, Bacteroides plebeius, versus lower proportions of unassigned Ruminococcaceae. Fermentations of P5, P6 and P9 (grouped in Figure 3A and Figure 4) differed by high levels of Lachnospira, Butyrivibrio and Coprococcus (unassigned). Clustering of P7 and P8 (Figures 3A,B and Figure 4) seemed to be caused by reduction of $F$. prausnitzii, along with highly increased abundances of unclassified Clostridiales, Coprococcus and Proteobacteria (Disulfovibrio D168, Citrobacter, Klebsiella, and Enterobacter). 


\section{Correlation between bacterial abundances and the structural properties of pectins}

Figure 5 shows correlations between the relative abundances of species-level OTUs and characteristics of pectins (content of monosaccharides, galacturonic acid, rhamnogalacturonan fraction, degree of esterification and degree of branching). The levels of Oscillospira, Blautia, Dorea, Ruminococcus, Coprococcus, $R$. torques, Lachnospiraceae and Clostridiales within phylum Firmicutes, and Paraprevotella, B. uniformis, B. ovatus, P. distasonis and Prevotella within phylum Bacteroidetes, correlated significantly ( $\mathrm{r}=0.35-0.71, \mathrm{p}<0.05$ ) or showed a tendency to increase with higher fractions of simple sugars (galactose, rhamnose, xylose and glucose) and lower content of galacturonic acid and DE values. Species F. prausnitzii and family Ruminococcaceae showed inverse correlation with the sugar residues, GalA and DE $(r=-0.42--0.72, \mathrm{p}<0.01)$, compared to bacterial taxa above. Abundance of $P$. copri correlated positively with arabinose $(\mathrm{r}=0.61, \mathrm{p}<0.001)$ and DE $(\mathrm{r}=0.77, \mathrm{p}<0.001)$ and negatively with degree of branching $(\mathrm{r}=-0.49, \mathrm{p}<0.01)$. Additionally, genera Coprococcus and Lachnospira correlated positively with $\mathrm{DBr}(\mathrm{r}=0.48-0.68, \mathrm{p}<0.01)$ and negatively $(r=-0.48--0.82, \mathrm{p}<0.01)$ with $\mathrm{DE}$ and arabinose.

\begin{tabular}{|c|c|c|c|c|c|c|c|c|c|}
\hline Gal & Rha & Xyl & Glc & Ara & RG & GalA & DE & DBr & \\
\hline 0.71 & 0.59 & 0.48 & 0.42 & & 0.61 & -0.54 & -0.68 & & g_Oscillospira \\
\hline 0.46 & 0.40 & 0.40 & 0.44 & & 0.48 & -0.45 & & & g_Blautia \\
\hline 0.38 & 0.35 & & & & 0.47 & -0.42 & & & g_Blautia; Other \\
\hline 0.55 & 0.53 & & 0.41 & & 0.57 & -0.54 & & & g_Dorea \\
\hline 0.59 & 0.52 & 0.41 & 0.47 & & 0.53 & -0.47 & -0.50 & & f_Lachnospiraceae \\
\hline 0.45 & 0.48 & & & & 0.41 & & -0.53 & & f_Lachnospiraceae;Other \\
\hline 0.52 & 0.35 & & & & 0.41 & & -0.55 & & g_Ruminococcus \\
\hline 0.60 & 0.46 & & & & 0.59 & -0.46 & -0.57 & & o_Clostridiales \\
\hline 0.36 & 0.38 & & & & 0.41 & -0.41 & & & s_[Ruminococcus] torques \\
\hline 0.57 & 0.38 & & & & 0.41 & & & & g_[Ruminococcus] \\
\hline \multirow[t]{3}{*}{0.44} & 0.52 & & & & 0.36 & & -0.82 & 0.48 & g_Coprococcus \\
\hline & & & & -0.53 & & & -0.48 & 0.58 & g_Coprococcus; Other \\
\hline & & & & -0.50 & -0.41 & & -0.51 & 0.68 & g_Lachnospira \\
\hline-0.70 & -0.63 & -0.51 & -0.47 & & -0.69 & 0.65 & 0.59 & & s_Faecalibacterium prausnitzii \\
\hline-0.64 & -0.75 & -0.42 & -0.42 & & -0.72 & 0.67 & 0.43 & & f_Ruminococcaceae \\
\hline 0.70 & 0.43 & 0.52 & 0.44 & & 0.60 & -0.54 & -0.46 & & s_Bacteroides uniformis \\
\hline 0.47 & & 0.49 & & -0.56 & 0.36 & & -0.59 & & s_Bacteroides ovatus \\
\hline 0.42 & 0.57 & & & & 0.49 & -0.42 & & & g_Paraprevotella \\
\hline \multirow[t]{3}{*}{0.38} & & 0.37 & & & & & -0.37 & & s_Parabacteroides distasonis \\
\hline & 0.38 & & & & & & -0.57 & & g_Prevotella \\
\hline & & & & 0.61 & & & 0.77 & -0.49 & s_Prevotella copri \\
\hline
\end{tabular}

Correlation coefficient

$-1 \quad 0 \quad+1$

Figure 5. Correlation between the relative abundances of bacterial taxa and the structural characteristics of pectins (P1 - P9), including content of galactose (Gal), rhamnose (Rha), xylose (Xyl), glucose (Glc), arabinose (Ara), and galacturonic acid (GalA), fraction of rhamnogalacturonan (RG), degree of branching (DBr) and degree of esterification (DE). 
Correlation analysis was performed with combined samples $(48,56$ and $72 \mathrm{~h}$ fermentation), obtained for each pectin in two independent fermentations. Significant correlation is indicated by the Spearman rank correlation coefficient (FDR-adjusted, $\mathrm{p}<$ 0.05).

\section{Production of short chain and branched chain fatty acids}

The cumulative production of SCFA (acetate, propionate and $n$-butyrate) after 72 $\mathrm{h}$ fermentation is shown in Table 2 . The amounts of total SCFA and acetate were not different between the pectins, comprising 131 - $162 \mathrm{mmol}$ and 81 - $95 \mathrm{mmol}$, respectively. Cumulative production of propionate was largest for sugar beet pectin (P4) (43.3 mmol) and RGI (P10) (47.6 mmol), and differed significantly from the citrus pectins P2, P5, P6, P7 and P9 (24.2 - $30.1 \mathrm{mmol})$. Generally, the HM pectins (P1, P2, P3 and P4) generated significantly higher $(\mathrm{p}<0.05)$ amounts of propionate and total SCFA compared to LM pectins (P5, P6, P7, P8 and P9). Production of butyrate was lowest in RGI (P10) fermentations (19.3 mmol) and statistically different from pectins P1, P2, P3 and P9 (27.9 - $32.9 \mathrm{mmol})$. The lowest acetate to propionate ratios $(2.0$ - 2.4) were determined for P4, P1 and P10 and highest (3.2 - 3.6) for P5 and P6 fermentations.

Table 2: Cumulative production of acetate, propionate, $n$-butyrate and total short chain fatty acids (SCFA) after $72 \mathrm{~h}$ fermentation of pectins in TIM- 2 colon $\operatorname{model}^{1}(\mathrm{mmol})$

\begin{tabular}{|c|c|c|c|c|c|}
\hline $\begin{array}{l}\text { Pectin } \\
\text { ID }\end{array}$ & Acetate & Propionate & n-butyrate & Total SCFA & $\begin{array}{l}\text { Ratio } \\
\text { Acetate/Propionate }\end{array}$ \\
\hline P1 & $86.6 \pm 1.6^{\mathrm{a}}$ & $36.1 \pm 2.3^{\mathrm{bc}}$ & $32.9 \pm 2.9^{b}$ & $155.6 \pm 6.7^{\mathrm{a}}$ & $2.4 \pm 0.1^{\mathrm{def}}$ \\
\hline P2 & $85.0 \pm 0.6^{\mathrm{a}}$ & $30.1 \pm 5.4^{\text {cd }}$ & $28.8 \pm 2.9^{\mathrm{bc}}$ & $143.8 \pm 1.9^{\mathrm{a}}$ & $2.9 \pm 0.5^{\text {abcd }}$ \\
\hline P3 & $86.8 \pm 3.2^{\mathrm{a}}$ & $32.7 \pm 1.5^{\mathrm{bcd}}$ & $27.9 \pm 1.5^{\mathrm{bc}}$ & $147.5 \pm 3.2^{\mathrm{a}}$ & $2.7 \pm 0.1^{\text {cdef }}$ \\
\hline P4 & $95.3 \pm 0.2^{\mathrm{a}}$ & $43.3 \pm 0.2^{\mathrm{ab}}$ & $24.0 \pm 1.5^{\mathrm{cd}}$ & $162.6 \pm 1.5^{\mathrm{a}}$ & $2.2 \pm 0.1^{\mathrm{efg}}$ \\
\hline P5 & $82.7 \pm 2.7^{\mathrm{a}}$ & $24.3 \pm 1.5^{\mathrm{d}}$ & $23.9 \pm 0.5^{\mathrm{cd}}$ & $131 .($ & $0.1^{\mathrm{ab}}$ \\
\hline P6 & $87.4 \pm 5.6^{\mathrm{a}}$ & $24.2 \pm 0$ & $26.7 \pm 0$ & 138. & 3.6 \\
\hline P7 & & & $4.1 \pm$ & $11.2^{\mathrm{a}}$ & \\
\hline P8 & $92.3 \pm 10.1^{\mathrm{a}}$ & $32.6 \pm 3$ & $21.9 \pm 3.5^{\text {ca }}$ & $146.7 \pm 17.0^{\mathrm{a}}$ & $2.8 \pm 0.1^{\text {bcde }}$ \\
\hline P9 & $80.8 \pm 2.4^{\mathrm{a}}$ & $25.4 \pm 2.1^{\mathrm{cd}}$ & $28.2 \pm 1.3^{\mathrm{bc}}$ & $134.3 \pm 5.7^{\mathrm{a}}$ & $3.2 \pm 0.2^{\mathrm{abc}}$ \\
\hline P10 & $92.5=$ & 2 & $19 .$. & 159 & \\
\hline SIEM & $58.8 \pm 3.0^{\mathrm{b}}$ & $1^{\text {bcd }}$ & 47.9 & $11.8^{\mathrm{a}}$ & \\
\hline $\mathrm{HM} / \mathrm{LM}^{2}$ & $88.4 / 87.3^{\mathrm{ns}}$ & $35.6 / 27.3^{* *}$ & $28.4 / 25.0^{\mathrm{ns}}$ & 152.4/139.6* & $2.55 / 3.23^{* *}$ \\
\hline \multicolumn{6}{|c|}{$\begin{array}{l}\text { 1Mean values ( } \pm \text { SD) in lumen and dialysate (combined) from two independent TIM-2 } \\
\text { experiments. Total SCFA is a sum of acetic, propionic and butyric acids. Different } \\
\text { superscripts within a column (a,b, c, d, e, fand g) indicate significant differences between } \\
\text { the substrates (one-way ANOVA with Tukey's post-hoc test, p < } 0.05 \text { ). Pectins are } \\
\text { denoted by codes described in Table } 1 \text {. SIEM was a basal simulated ileal efflux medium } \\
\text { used for the control TIM- } 2 \text { experiments. } \\
{ }^{2} \text { Amounts of SCFA (means) produced by HM pectins (P1, P2, P3 and P4) versus LM } \\
\text { pectins (P5, P6, P7, P8 and P9). Significant differences between the HM and LM pectins } \\
\text { were determined by the two-sided Wilcoxon rank sum test }\left({ }^{*} p<0.05 ;{ }^{* *} p<0.01 \text {; ns - }\right. \\
\text { insignificant). }\end{array}$} \\
\hline
\end{tabular}


Production of $i$-butyrate, $i$-valerate and total BCFA were slightly lower in RGI fermentations, though not significantly different from other treatments (Supplementary Table S2). The amount of propionate throughout fermentation $(24-72 \mathrm{~h})$ correlated positively with $P$. copri, $R$. torques and unclassified Clostridiales, and, negatively, with genera Coprococcus, Butyrivibrio and Lachnospira (Table 3). Production of butyrate was reduced with increasing abundances of B. ovatus and Bacteroides after $48 \mathrm{~h}$ fermentation. Correlation between other bacterial taxa and SCFA was insignificant.

Table 3: Correlation between the relative abundances of bacterial taxa and cumulative production of the short chain fatty acids (SCFA) propionate and butyrate after 24, 48 and $72 \mathrm{~h}$ fermentation of pectins in TIM-2 colon model

\begin{tabular}{cllll}
\hline \multirow{2}{*}{ SCFA } & \multicolumn{3}{c}{ Bacterial taxa } & \multicolumn{3}{c}{ Correlation coefficient, $\mathrm{r}^{1}$} \\
\cline { 3 - 5 } Propionate & s_Prevotella copri & \multicolumn{1}{c}{$24 \mathrm{~h}$} & $48 \mathrm{~h}$ & \multicolumn{1}{c}{$72^{\mathrm{h}}$} \\
& g_[Ruminococcus];Other & $0.48 \mathrm{~ns}$ & $0.71^{* *}$ & $0.61^{* *}$ \\
& s_[Ruminococcus] torques & $0.62^{*}$ & $0.64^{*}$ & $0.67^{*}$ \\
& o_Clostridiales;Other & $0.39 \mathrm{~ns}$ & $0.61^{*}$ & $0.62^{*}$ \\
& f_Ruminococcaceae;Other & $-0.66^{*}$ & $-0.69^{* *}$ & -0.51 \\
& g_Coprococcus;Other & $-0.73^{* *}$ & $-0.32 \mathrm{~ns}$ & $-0.72^{* *}$ \\
& g_Butyrivibrio & $-0.64^{*}$ & $-0.73^{* *}$ & $-0.61^{*}$ \\
& g_Lachnospira & $-0.70^{* *}$ & $-0.80^{* * *}$ & $-0.77^{* * *}$ \\
& s_Bacteroides ovatus & $-0.53 \mathrm{~ns}$ & $-0.79^{* *}$ & $-0.66^{*}$ \\
& g_Bacteroides & $-0.57 \mathrm{~ns}$ & $-0.64^{*}$ & $-0.40 \mathrm{~ns}$ \\
\hline
\end{tabular}

${ }^{1}$ Spearman rank correlation coefficient (r); asterisks denote significant correlation (FDRadjusted, ${ }^{*} \mathrm{p}<0.05$; ${ }^{* *} \mathrm{p}<0.01$; and $\left.{ }^{* * *} \mathrm{p}<0.001\right)$; ns - insignificant.

\section{Discussion}

\section{Changes in bacterial abundances was related to species-specific utilization of pectins}

In this study we conducted TIM-2 fermentations with diverse pectins to investigate the relationship between the structural properties of pectins and the changes in microbiota composition. We expected that enrichment or reduction of bacterial populations during fermentations would depend on their ability to degrade pectins, and/or utilize POS and other metabolites (cross-feeding interactions). Observed increases in abundances of pectin-degrading bacteria within the order Bacteroidales, genera Prevotella, Oscillospira, Clostridium, F. prausnitzii and family Enterobacteriaceae was likely due to pectin utilization 
favouring their growth $(22,32-34)$. Interestingly, shifts in related species of Bacteroides ovatus, B. plebeius and B. uniformis depended on the substrate, which can likely be explained by the species-specific activity of pectin-degrading enzymes and the hierarchical preference of substrate utilization. Accordingly, Tuncil and coworkers demonstrated that human gut symbionts $B$. thetaiotamicron and B. ovatus, grown together on pectic oligosaccharides, had inverse growth profile, concurrent with a different expression pattern of glycan utilization genes (35). Preferential utilization of metabolites from pectin degradation can be expected in this study, regarding the highly competitive environment in TIM-2. High increases in the genera Butyrivibrio and Lachnospira, previously shown incapable to break down pectins $(36,37)$, might be linked to metabolic cross-feeding interactions between the members of the faecal microbiota.

\section{Health-promoting bacterial populations were selectively modified by pectins}

Various pectin-mediated shifts in this study can be considered as beneficial, regarding the current knowledge of microbial dysbiosis, related to obesity and IBD. Thus, it was previously shown that obesity-related bias in the gut microbiota included higher levels of Blautia, Eubacterium, Roseburia, Dorea and Ruminococcus, along with reduction of $F$. prausnitzii, Oscillospira, Christensenellaceae, Prevotella, Bacteroides and genera within Proteobacteria (34,38-42). Besides, R. torques and R. gnavus, decreased in this study, were found in higher numbers in IBD patients (43). Notably, shifts in F. prausnitzii, Coprococcus, B. ovatus, B. plebeius, P. copri and Sutterella were strongly dependent on the type of pectin, suggesting that these species can be modulated by usage of specific pectins. Among them, F. prausnitzii, is commonly referred to as a marker for intestinal health, exhibiting anti-inflammatory effects in the gut (44). This study indicated that stimulation of $F$. prausnitzii could be achieved by fermentation of HM pectins (P1 - P3) rather than LM pectins (P7 and P8). In their turn, LM citrus pectins (P5 - P9) were efficient to decrease proportions of $P$. copri, a microbe associated with induced insulin resistance in mice and rheumatoid arthritis in humans $(45,46)$. However, in other studies, $P$. copri was referred to as a beneficial microbe, as it was enriched in the colon of healthy subjects, who exhibited improved glucose metabolism following consumption of barley-based bread (47). LM citrus pectins (P5 - P9) were found to increase the levels of Coprococcus (mostly P7), which is linked to reduced severity of IBS and autism in humans $(48,49)$. An increase in B. plebeius, selectively stimulated by 
SBP (P4) and RGI (P10) in this study, is known to be related to remission pattern of microbiota in Crohn's disease patients (50).

\section{Production of propionate, butyrate and total SCFA differed between the pectins}

The overall production of propionate was highest in RGI (P10) fermentations and in fermentations of HM pectins (especially SBP [P4]) and correlated positively with the relative abundances of $P$. copri, Ruminococcus spp, and unidentified Clostridiales, suggesting that these species were able to generate propionate from pectin. Supporting our results, Gulfi and coworkers reported that HM pectins had a tendency to produce larger amounts of propionic acid in batch fecal fermentations, compared to LM pectins (51). Additionally, the ability of Prevotella and Ruminococcus to produce propionate was recently confirmed by genomic analysis of colonic anaerobes combined with growth experiments $(52,53)$. Production of propionate by Ruminococcus is known to be enhanced in the presence of rhamnose and fucose (53), which are the common structural units of pectin molecules. Consequently, increased production of propionate in SBP (P4) and RGI (P10) fermentations might be related to the relatively higher content of rhamnose and other neutral sugars. Interestingly, the acetate to propionate ratios were found to be reduced in RGI (P10) and the HM pectin group, lowest for SBP (P4) and harsh extracted pectin from orange (P1). Lower acetate/propionate ratios have been associated with an anti-cholesterolemic effect and reduction of cardiovascular disease risk, and are generally considered as beneficial (54). The lowest amount of butyrate was detected in RGI (P10) fermentations, which might be explained by depletion of $F$. prausnitzii, a predominant butyrate producer in the gut (55). The negative correlation between butyrate and the numbers of Bacteroides spp. was probably related to their inability to produce butyrate (55). Production of SCFA in fermentations of citrus pectins (P1 - P3 and P5 - P9) was not statistically different, despite the differences in microbiota composition. This effect was presumably caused by the functional redundancy of microbial populations and the metabolic cross-feeding interactions (17).

\section{Changes in microbiota were related to the structural features of pectins.}

Shifts in bacterial abundances in TIM-2 fermentations were related to the structural features of pectins. We identified at least five factors essential for microbiota shaping: (i) degree of esterification of polygalacturonic acid, (ii) composition of neutral sugars, (iii) distribution of HG and RG fractions, (iv) 
degree of branching and (v) modification of pectic backbone, e.g., by amidation. Degree of esterification is probably the most important parameter, as seen from the beta-diversity analysis and the correlation of bacterial taxa with DE. So far, only few studies indicated a link between the DE in pectins and microbiota composition. In agreement with our results, enrichment in Prevotella spp. was found in colonic microbiota of pigs fed with LM pectins, while genus Bacteroides was increased in fecal batch fermentations of LM pectin contrary to HM pectin $(23,25)$. Results of the correlation analysis (Figure 5) pointed out the possibility of differential stimulation of bacterial populations using pectins with different sugar content, especially rhamnose and galactose. Thus, correlation of $F$. prausnitzii with the major sugars (negative) and GalA/DE (positive) suggested, that HM pectins with high fraction of HG over RG would be preferable for growth of this beneficial microbe. Interestingly, arabinose differed from other sugars, correlating positively with $P$. copri and negatively with Coprococcus and Lachnospira. Both Lachnospira spp. and $P$. copri possess $\alpha$-arabinofuranosidase activity (56), having the potential to degrade arabinan side chains in pectin molecules. Thus, this inverse correlation is most probably linked to the differences between bacterial species in activity of arabinanolytic enzymes and the metabolic hierarchy as discussed above. Unexpectedly, we observed similarity in microbiota profiling between the structurally different substrates, sugar beet pectin (P4) and RGI (P10). This similarity might be associated with HG/RG distribution, as both SBP and RGI were distinguished by the low HG (GalA) and high RG content compared to other pectins. Similarity between pectins P7 and P8 is most probably attributed to amidation of the C-6 uronate groups in addition to the equally low DE values. It is well-known that structural features of pectins affect their rheological behavior and functional properties, e.g., gelling capacity, viscosity, molecular conformation and solubility (2). We suppose that these properties might also have an impact on substrate-bacterial interactions and pectin utilization in microbiota fermentations. Furthermore, carboxyl groups in the polygalacturonic chains can be involved in electrostatic interactions and, together with amide groups, in hydrogen bonding. In a previous study, we observed that the surface charge (or zeta-potential) of the LM pectins, was lower than that of HM pectins, due to the higher fraction of non-esterified carboxyl groups (8). Lower zeta-potential in LM pectins indicated stronger electrostatic repulsion upon interactions with bacterial cell wall groups (hydroxyl, carboxyl, etc.) negatively charged at neutral $\mathrm{pH}$ (57). High degree of branching and RG fraction can either provide steric hindrance or contribute to hydrophobic interactions with non-polar groups in bacterial cell walls. Involvement in the different types of interactions could explain the opposite 
correlation found for DE (and galacturonic acid) compared to $\mathrm{DBr}$ and neutral sugars with bacterial taxa in this study. Differences in the distribution of structural groups would govern molecular conformation in pectins and, thus, exposure of carbohydrate chains to enzymatic cleavage and interactions with bacterial cell surface molecules. It may, consequently, result in different rates of substrate utilization and metabolite profiles, and drive pectin-specific differences in microbiota shaping.

In conclusion, this study provided evidence that modulation of the gut microbiota by pectins depended on their structural features. We identified specific bacterial taxa which abundances were differentially affected by pectins and proposed the main factors, linked to the differences in microbiota composition. Understanding of the interplay between the gut commensals and the structural properties of pectins is essential to predict physiological effects of ingested pectins and to provide ideas for development of pectin-containing dietary fibres, targeting beneficial bacteria to facilitate more balanced microbiota profiles. It can be also relevant to verify our findings in relation to other microbiotas, e.g., from diseased subjects. Additionally, further comparative in vitro and in vivo studies with structurally diverse pectins and their derivatives are needed to achieve detailed knowledge of structure-function relationship of pectins in the gut.

\section{Availability of data and material}

The metadata have been deposited in the European Nucleotide Archive (ENA) database [accession number: PRJEB25646].

\section{Conflict of interest}

The authors declare no conflict of interest.

\section{Funding}

The research was funded by the Danish Council for Strategic Research (DSR, project BioSyn, no. 3050-00005B) within the frame of the project "Strategic Research Collaboration in Food Science in the State of São Paulo, Brazil and Denmark" and supported by the Brazilian National Council for Scientific and Technological Development (CNPq-Brazil), the Program "Science without Borders". From the CNPq-Brazil Carlota Bussolo de Souza received a scholarship 246027/2012-6, and Thiago Cahu - scholarship 233521/2014-3. This research has additionally been supported by the Dutch Province of Limburg. 


\section{Acknowledgments}

The authors thank technician Wendy Borst (Maastricht University, Venlo) for assistance in the TIM-2 experiments.

\section{Author contributions}

LJ, NL, KV and AB conceived, planned and coordinated the study; KV, CBS performed fermentations, sample collection and SCFA analysis; NL conducted microbiota analysis and wrote manuscript; LK and WK performed sequencing and bioinformatics; KMH and TBC participated in production and characterization of pectins; MW advised in statistical analysis. All authors read and approved the final manuscript. 


\section{References}

1. Regulation (EC) No $1333 / 2008$ of the European Parliament and of the Council of 16 December 2008 on food additives. Off J Eur Union. 2008;L 354:16-33.

2. Sila DN, Van Buggenhout S, Duvetter T, Van Loey A, Hendrickx M. Pectins in processed fruits and vegetables: Part II-Structure function relationships. Compr Rev Food Sci Food Saf. 2009;8(2):105-17.

3. Chan SY, Choo WS, Young DJ, Loh XJ. Pectin as a rheology modifier: Origin, structure, commercial production and rheology. Carbohydr Polym. 2017;161:118-39.

4. Fukunaga T, Sasaki M, Araki Y, Okamoto T, Yasuoka T, Tsujikawa T, et al. Effects of the soluble fibre pectin on intestinal cell proliferation, fecal short chain fatty acid production and microbial population. Digestion. 2003;67:42-9.

5. Shinohara K, Ohashi Y, Kawasumi K, Terada A, Fujisawa T. Effect of apple intake on fecal microbiota and metabolites in humans. Anaerobe. 2010;16:510-5.

6. Schwartz SE, Levine RA, Weinstock RS, Petokas S, Mills CA, Thomas FD. Sustained pectin ingestion: Effect on gastric emptying and glucose tolerance in non-insulin-dependent diabetic patients. Am J Clin Nutr. 1988;48:1413-7.

7. Parkar SG, Redgate EL, Wibisono R, Luo X, Koh ETH, Schröder R. Gut health benefits of kiwifruit pectins: Comparison with commercial functional polysaccharides. J Funct Foods. 2010;2:210-8.

8. Larsen N, Cahú TB, Marta S, Saad I, Blennow A, Jespersen L. The effect of pectins on survival of probiotic Lactobacillus spp. in gastrointestinal juices is related to their structure and physical properties. Food Microbiol. 2018;74:11-20.

9. Sulek K, Vigsnaes LK, Schmidt LR, Holck J, Frandsen HL, Smedsgaard J, et al. A combined metabolomic and phylogenetic study reveals putatively prebiotic effects of high molecular weight arabino-oligosaccharides when assessed by invitro fermentation in bacterial communities derived from humans. Anaerobe. 2014;28:68-77.

10. Gómez B, Gullón B, Yáñez R, Schols H, Alonso JL. Prebiotic potential of pectins and pectic oligosaccharides derived from lemon peel wastes and sugar beet pulp: A comparative evaluation. J Funct Foods. 2016;20:10821.

11. Manderson K, Pinart M, Tuohy KM, Grace WE, Hotchkiss AT, Widmer W, et al. In vitro determination of prebiotic properties of oligosaccharides derived from an orange juice manufacturing by-product stream. Appl Environ Microbiol. 2005;71(12):8383-9.

12. Leijdekkers AGM, Aguirre M, Venema K, Bosch G, Gruppen H, Schols HA. In vitro fermentability of sugar beet pulp derived oligosaccharides using human and pig fecal inocula. J Agric Food Chem. 2014;62(5):1079-87.

13. Onumpai C, Kolida S, Bonnin E, Rastall RA. Microbial utilization and 
selectivity of pectin fractions with various structures. Appl Environ Microbiol. 2011;77(16):5747-54.

14. Aguirre M, Jonkers DMAE, Troost FJ, Roeselers G, Venema K. In vitro characterization of the impact of different substrates on metabolite production, energy extraction and composition of gut microbiota from lean and obese subjects. PLoS One. 2014;9(11):e113864.

15. Chen J, Liang RH, Liu W, Li T, Liu CM, Wu SS, et al. Pectic-oligosaccharides prepared by dynamic high-pressure microfluidization and their in vitro fermentation properties. Carbohydr Polym. 2013;91(1):175-82.

16. Gómez B, Gullón B, Remoroza C, Schols HA, Parajó JC, Alonso JL. Purification, characterization, and prebiotic properties of pectic oligosaccharides from orange peel wastes. J Agric Food Chem. 2014;62:9769-82.

17. Reichardt N, Vollmer M, Holtrop G, Farquharson FM, Wefers D, Bunzel M, et al. Specific substrate-driven changes in human faecal microbiota composition contrast with functional redundancy in short-chain fatty acid production. ISME J. 2018 Feb;12(2):610-22.

18. Martens EC, Lowe EC, Chiang H, Pudlo NA, Wu M, McNulty NP, et al. Recognition and degradation of plant cell wall polysaccharides by two human gut symbionts. PLoS Biol. 2011;9(12):e1001221.

19. Grondin JM, Tamura K, Déjean G, Abbott DW, Brumer H. Polysaccharide utilization loci: Fueling microbial communities. J Bacteriol. 2017;199(15):e00860-16.

20. Wicker L, Kim Y, Kim MJ, Thirkield B, Lin Z, Jung J. Pectin as a bioactive polysaccharide - Extracting tailored function from less. Food Hydrocoll. 2014;42(P2):251-9.

21. Vogt LM, Sahasrabudhe NM, Ramasamy U, Meyer D, Pullens G, Faas MM, et al. The impact of lemon pectin characteristics on TLR activation and T84 intestinal epithelial cell barrier function. J Funct Foods. 2016;22:398407.

22. Olano-Martin E, Gibson GR, Rastall RA. Comparison of the in vitro bifidogenic properties of pectins and pectic-oligosaccharides. J Appl Microbiol. 2002;93(3):505-11.

23. Dongowski G, Lorenz A, Proll J. The degree of methylation influences the degradation of pectin in the intestinal tract of rats and in vitro. J Nutr. 2002 Jul;132(7):1935-44.

24. Li P, Xia J, Nie Z, Shan Y. Pectic oligosaccharides hydrolyzed from orange peel by fungal multi-enzyme complexes and their prebiotic and antibacterial potentials. LWT - Food Sci Technol. 2016;69:203-6.

25. Tian L, Bruggeman G, van den Berg M, Borewicz K, Scheurink AJW, Bruininx $\mathrm{E}$, et al. Effects of pectin on fermentation characteristics, carbohydrate utilization, and microbial community composition in the gastrointestinal tract of weaning pigs. Mol Nutr Food Res. 2017;61(1):1600186.

26. Yapo B, Koffi K. Extraction and characterization of highly gelling low 
methoxy pectin from cashew apple pomace. Foods. 2013;3:1-12.

27. Venema K. The TNO In Vitro Model of the Colon ( TIM-2 ). In: Verhoeckx $\mathrm{K}$, Cotter P L-EI, editor. The Impact of Food Bioactives on Health: in vitro and ex vivo models. Cham (CH): 2015. p. 293-304.

28. Thakur BR, Singh RK, Handa AK. Chemistry and Uses of Pectin - A Review. Crit Rev Food Sci Nutr. 1997;37(1):47-73.

29. Williams AR, Hansen TVA, Krych L, Ahmad HF Bin, Nielsen DS, Skovgaard $\mathrm{K}$, et al. Dietary cinnamaldehyde enhances acquisition of specific antibodies following helminth infection in pigs. Vet Immunol Immunopathol. 2017;189:43-52.

30. Caporaso JG, Kuczynski J, Stombaugh J, Bittinger K, Bushman FD, Costello $\mathrm{EK}$, et al. QIIME allows analysis of high-throughput community sequencing data. Vol. 7, Nature methods. United States; 2010. p. 335-6.

31. Mcdonald D, Price MN, Goodrich J, Nawrocki EP, Desantis TZ, Probst A, et al. An improved Greengenes taxonomy with explicit ranks for ecological and evolutionary analyses of bacteria and archaea. ISME J. 2012;6(3):610-8.

32. Abbott DW, Boraston AB. Structural biology of pectin degradation by Enterobacteriaceae. Microbiol Mol Biol Rev. 2008;72(2):301-16.

33. Lopez-Siles M, Khan TM, Duncan SH, Harmsen HJM, Garcia-Gil LJ, Flint HJ. Cultured representatives of two major phylogroups of human colonic Faecalibacterium prausnitzii can utilize pectin, uronic acids, and hostderived substrates for growth. Appl Environ Microbiol. 2012;78(2):4208.

34. Tims S, Derom C, Jonkers DM, Vlietinck R, Saris WH, Kleerebezem M, et al. Microbiota conservation and BMI signatures in adult monozygotic twins. ISME J. 2013;7:707-17.

35. Tuncil YE, Nakatsu CH, Kazem AE, Arioglu-Tuncil S, Reuhs B, Martens EC, et al. Delayed utilization of some fast-fermenting soluble dietary fibers by human gut microbiota when presented in a mixture. J Funct Foods [Internet]. 2017 May;32:347-57.

36. Flint HJ, Scott KP, Duncan SH, Louis P, Forano E. Microbial degradation of complex carbohydrates in the gut. Vol. 3, Gut Microbes. 2012. p. 289-306.

37. van Laere KMJ, Hartemink R, Bosveld M, Schols HA, Voragen AGJ. Fermentation of plant cell wall derived polysaccharides and their corresponding oligosaccharides by intestinal bacteria. J Agric Food Chem. 2000;48:1644-52.

38. Goodrich JK, Waters JL, Poole AC, Sutter JL, Koren O, Blekhman R, et al. Human genetics shape the gut microbiome. Cell Novemb. 2014;159(4):789-99.

39. Nadal I, Santacruz A, Marcos A, Warnberg J, Garagorri M, Moreno L, et al. Shifts in clostridia, bacteroides and immunoglobulin- coating fecal bacteria associated with weight loss in obese adolescents. Int J Obes. 2009;33(10):758-67.

40. Verdam FJ, Fuentes S, De Jonge C, Zoetendal EG, Erbil R, Greve JW, et al. 
Human intestinal microbiota composition is associated with local and systemic inflammation in obesity. Obesity. 2013;21:E607-15.

41. Furet JP, Kong LC, Tap J, Poitou C, Basdevant A, Bouillot JL, et al. Differential adaptation of human gut microbiota to bariatric surgeryinduced weight loss: Links with metabolic and low-grade inflammation markers. Diabetes. 2010;59:3049-57.

42. Kasai C, Sugimoto K, Moritani I, Tanaka J, Oya Y, Inoue H, et al. Comparison of the gut microbiota composition between obese and non-obese individuals in a Japanese population, as analyzed by terminal restriction fragment length polymorphism and next-generation sequencing. BMC Gastroenterol. 2015;15(1):100.

43. Png CW, Lindén SK, Gilshenan KS, Zoetendal EG, McSweeney CS, Sly LI, et al. Mucolytic bacteria with increased prevalence in IBD mucosa augment in vitro utilization of mucin by other bacteria. Am J Gastroenterol. 2010;105:2420-8.

44. Walters WA, Xu Z, Knight R. Meta-analyses of human gut microbes associated with obesity and IBD. FEBS Lett. 2014 Nov;588(22):4223-33.

45. Pedersen HK, Gudmundsdottir V, Nielsen HB, Hyotylainen T, Nielsen T, Jensen $\mathrm{BAH}$, et al. Human gut microbes impact host serum metabolome and insulin sensitivity. Nature. 2016;535:376-81.

46. Pianta A, Arvikar S, Strle K, Drouin EE, Wang Q, Costello CE, et al. Evidence of the immune relevance of prevotella copri, a gut microbe, in patients with rheumatoid arthritis. Arthritis Rheumatol. 2017;69(5):964-75.

47. Kovatcheva-Datchary P, Nilsson A, Akrami R, Lee YS, De Vadder F, Arora $\mathrm{T}$, et al. Dietary fiber-induced improvement in glucose metabolism is associated with increased abundance of Prevotella. Cell Metab [Internet]. 2015 Dec 1;22(6):971-82.

48. Tap J, Derrien M, Törnblom H, Brazeilles R, Cools-portier S, Doré J, et al. Identification of an intestinal microbiota signature associated with severity of Irritable Bowel Syndrome. Gastroenterology. 2017;152(1):111-23.

49. Kang D, Park JG, Ilhan ZE, Wallstrom G, Labaer J, Adams JB, et al. Reduced incidence of Prevotella and other fermenters in intestinal microflora of autistic children. 2013;8(7):e68322.

50. Mondot S, Lepage P, Seksik P, Allez M, Tréton X, Bouhnik Y, et al. Structural robustness of the gut mucosal microbiota is associated with Crohn's disease remission after surgery. Gut. 2016;65:954-62.

51. Gulfi M, Arrigoni E, Amadò R. Influence of structure on in vitro fermentability of commercial pectins and partially hydrolysed pectin preparations. Carbohydr Polym. 2005;59:247-55.

52. Chen T, Long W, Zhang C, Liu S, Zhao L, Hamaker BR. Fiber-utilizing capacity varies in Prevotella- versus Bacteroides-dominated gut microbiota. Sci Rep. 2017;7:2594.

53. Reichardt N, Duncan SH, Young P, Belenguer A, Leitch CM, Scott KP, et al. Phylogenetic distribution of three pathways for propionate production 
within the human gut microbiota. ISME J. 2014;8(10):1323-35.

54. den Besten G, van Eunen K, Groen AK, Venema K, Reijngoud D-J, Bakker $\mathrm{BM}$. The role of short-chain fatty acids in the interplay between diet, gut microbiota, and host energy metabolism. J Lipid Res. 2013;54:2325-40.

55. Louis P, Flint HJ. Formation of propionate and butyrate by the human colonic microbiota. Environ Microbiol. 2017;19(1):29-41.

56. Dodd D, Mackie RI, Cann IKO. Xylan degradation, a metabolic property shared by rumen and human colonic Bacteroidetes. Mol Microbiol. 2011;79(2):292-304.

57. Jiang W, Saxena A, Song B, Ward BB, Beveridge TJ, Myneni SCB. Elucidation of functional groups on gram-positive and gram-negative bacterial surfaces using Infrared spectroscopy. Langmuir. 2004;20:11433-42. 


\section{Supplemental material}

Supplementary Table S1: Structural properties of pectins used in this study (\%) ${ }^{1}$

\begin{tabular}{|c|c|c|c|c|c|c|c|c|c|c|}
\hline ID & $\mathrm{DE}$ & ialA & Gal & Ara & Rha & Glc & Xyl & $\mathrm{HG}$ & RG & $\mathrm{DBr}$ \\
\hline P1 & 58.8 & $\begin{array}{c}66.18 \pm \\
1.94 \mathrm{bc}\end{array}$ & $\begin{array}{l}18.73 \pm \\
0.73 \mathrm{abc}\end{array}$ & $\begin{array}{l}2.81 \pm \\
0.41^{\mathrm{d}}\end{array}$ & $\begin{array}{l}2.64 \pm \\
0.19 \text { bcd }\end{array}$ & $\begin{array}{c}8.73 \pm \\
0.48^{\mathrm{a}}\end{array}$ & $\begin{array}{l}0.63 \pm \\
0.03 \mathrm{bc}\end{array}$ & $\begin{array}{l}63.54 \pm \\
1.60 \mathrm{bc}\end{array}$ & $\begin{array}{c}26.82^{ \pm} \\
1.92^{c}\end{array}$ & $\begin{array}{l}14.9 \pm \\
0.3^{\mathrm{ab}}\end{array}$ \\
\hline P2 & 70.0 & $\begin{array}{c}73.10 \pm \\
2.24 \mathrm{ab}\end{array}$ & & $\begin{array}{l}7.10 \pm \\
0.21 \text { bcd }\end{array}$ & $\begin{array}{l}1.79 \pm \\
0.12^{\text {cd }}\end{array}$ & & $\begin{array}{l}0.25 \pm \\
0.01^{\mathrm{d}}\end{array}$ & $\begin{array}{c}71.30 \pm \\
0.49^{\mathrm{b}}\end{array}$ & $\begin{array}{c}25.47^{ \pm} \\
1.88^{c}\end{array}$ & $\begin{array}{c}10.8 \pm \\
0.2^{\mathrm{b}}\end{array}$ \\
\hline P3 & 74.7 & $\begin{array}{c}70.67 \pm \\
1.70^{\mathrm{b}}\end{array}$ & $\begin{array}{c}10.42^{ \pm} \\
0.39^{\mathrm{c}}\end{array}$ & $\begin{array}{c}15.18 \pm \\
0.20^{\mathrm{bc}}\end{array}$ & $\begin{array}{l}1.50 \pm \\
0.19^{d}\end{array}$ & $\begin{array}{l}1.14 \pm \\
0.20^{\mathrm{c}}\end{array}$ & $\begin{array}{l}0.29 \pm \\
0.01^{\mathrm{d}}\end{array}$ & $\begin{array}{c}69.17^{ \pm} \\
0.17^{\mathrm{b}}\end{array}$ & $\begin{array}{c}28.60 \pm \\
1.48^{\mathrm{c}}\end{array}$ & $\begin{array}{l}7.4 \pm \\
1.0^{\mathrm{b}}\end{array}$ \\
\hline P4 & 59. & $\begin{array}{c}46.91 \pm \\
3.96^{\mathrm{d}}\end{array}$ & $\begin{array}{c}27.95 \pm \\
1.78^{\mathrm{ab}}\end{array}$ & $\begin{array}{c}16.67 \pm \\
1.42^{\mathrm{b}}\end{array}$ & $\begin{array}{l}3.05 \pm \\
0.12^{\mathrm{abc}}\end{array}$ & $\begin{array}{l}3.66 \pm \\
0.19 \mathrm{bc}\end{array}$ & $\begin{array}{l}0.51 \pm \\
0.02 \mathrm{bc}\end{array}$ & $\begin{array}{c}43.86 \pm \\
2.29^{d}\end{array}$ & $\begin{array}{c}50.72 \pm \\
2.69^{\mathrm{b}}\end{array}$ & $\begin{array}{l}7.9 \pm \\
1.0^{\mathrm{b}}\end{array}$ \\
\hline P5 & 11.4 & $\begin{array}{c}59.88 \pm \\
0.59^{c}\end{array}$ & $\begin{array}{c}26.83 \pm \\
0.67 \mathrm{ab}\end{array}$ & $\begin{array}{l}2.80 \pm \\
0.09 \mathrm{~d}\end{array}$ & & & & $\begin{array}{c}56.16 \pm \\
1.02^{\mathrm{c}}\end{array}$ & & $\begin{array}{c}15.6 \pm \\
1.6^{\mathrm{ab}}\end{array}$ \\
\hline P6 & 31.8 & $\begin{array}{c}82.03 \pm \\
7.10^{a}\end{array}$ & $\begin{array}{l}9.07 \pm \\
0.93^{c}\end{array}$ & $\begin{array}{l}4.25 \pm \\
0.29 \mathrm{~cd}\end{array}$ & $\begin{array}{l}1.77 \pm \\
0.13^{\mathrm{cd}}\end{array}$ & $\begin{array}{c}0.70 \pm \\
0.29^{c}\end{array}$ & $\begin{array}{c}0.21 \pm \\
0.01^{\mathrm{d}}\end{array}$ & $\begin{array}{c}81.25 \pm \\
3.56^{\mathrm{a}}\end{array}$ & $\begin{array}{c}16.87 \pm \\
1.20^{\mathrm{d}}\end{array}$ & $\begin{array}{c}19.2 \pm \\
2.8^{\mathrm{a}}\end{array}$ \\
\hline P7 & 28.8 & $\begin{array}{c}67.30 \pm \\
1.88 \mathrm{bc}\end{array}$ & $\begin{array}{c}22.03 \pm \\
0.45^{\mathrm{abc}}\end{array}$ & $\begin{array}{l}3.14 \pm \\
0.17 \mathrm{~cd}\end{array}$ & $\begin{array}{l}2.86 \pm \\
0.24 \text { bcd }\end{array}$ & $\begin{array}{l}2.71 \pm \\
0.18 \mathrm{bc}\end{array}$ & $\begin{array}{l}0.39 \pm \\
0.02 \text { cd }\end{array}$ & $\begin{array}{c}64.45 \pm \\
1.14 \mathrm{bc}\end{array}$ & $\begin{array}{c}30.87 \pm \\
0.32^{c}\end{array}$ & $\begin{array}{l}15.1 \pm \\
2.5^{\mathrm{ab}}\end{array}$ \\
\hline P8 & 29.5 & $\begin{array}{c}52.91 \pm \\
0.25^{\mathrm{cd}}\end{array}$ & $\begin{array}{c}30.67 \pm \\
2.60^{\mathrm{a}}\end{array}$ & $\begin{array}{l}2.80 \pm \\
0.14^{\mathrm{d}}\end{array}$ & $\begin{array}{l}2.41 \pm \\
0.34 \text { bcd }\end{array}$ & $\begin{array}{c}8.48 \pm \\
0.70^{a}\end{array}$ & $\begin{array}{l}0.80 \pm \\
0.07^{b}\end{array}$ & $\begin{array}{c}50.50 \pm \\
0.18^{\mathrm{cd}}\end{array}$ & $\begin{array}{c}38.29 \pm \\
2.57 \mathrm{bc}\end{array}$ & $\begin{array}{l}8.3 \pm \\
0.1^{b}\end{array}$ \\
\hline P9 & 35.6 & $\begin{array}{c}72.95 \pm \\
2.22^{\mathrm{ab}}\end{array}$ & $\begin{array}{c}21.22 \pm \\
0.37 \mathrm{abc}\end{array}$ & $\begin{array}{l}0.82 \pm \\
0.22^{\mathrm{d}}\end{array}$ & $\begin{array}{l}1.72 \pm \\
0.15^{\text {cd }}\end{array}$ & $\begin{array}{l}1.52 \pm \\
0.31^{\mathrm{c}}\end{array}$ & $\begin{array}{l}0.49 \pm \\
0.02^{\text {bcd }}\end{array}$ & $\begin{array}{c}71.24 \pm \\
0.57^{\mathrm{b}}\end{array}$ & $\begin{array}{c}25.47 \pm \\
1.79^{c}\end{array}$ & $\begin{array}{c}10.4 \pm \\
1.8^{\mathrm{b}}\end{array}$ \\
\hline P10 & nd & $\begin{array}{c}11.02 \pm \\
1.10^{\mathrm{e}} \\
\end{array}$ & $\begin{array}{r}7.92 \pm \\
4.44^{\mathrm{c}} \\
\end{array}$ & $\begin{array}{c}69.92 \pm \\
4.98^{\mathrm{a}} \\
\end{array}$ & $\begin{array}{r}6.62 \pm \\
0.98^{\mathrm{a}} \\
\end{array}$ & $\begin{array}{l}1.67 \pm \\
0.58^{\mathrm{bc}}\end{array}$ & $\begin{array}{r}1.78 \pm \\
0.09^{\mathrm{a}} \\
\end{array}$ & $\begin{array}{l}4.41 \pm \\
2.72 \mathrm{e} \\
\end{array}$ & $\begin{array}{c}91.08 \pm \\
2.50^{\mathrm{a}} \\
\end{array}$ & $\begin{array}{l}8.5 \pm \\
1.2^{\mathrm{b}}\end{array}$ \\
\hline
\end{tabular}

1 DE: degree of esterification; Monosaccharides: Galacturonic acid (GalA), arabinose (Ara), rhamnose (Rha), galactose (Gal), glucose (Glc) and xylose (Xyl) were quantified by high performance anion exchange chromatography equipped with a PA20 column (HPAEC-PAD) and presented by mean values \pm SD from two repeats. Calculated values: homogalacturonan, $\mathrm{HG} \%=\mathrm{GalA} \%-\mathrm{Rha} \%$, rhamnogalacturonan, $\mathrm{RG} \%=2 \mathrm{Rha} \%+\mathrm{Ara} \%$ + Gal\%, and degree of branching DBr\% $=100 \% \times$ Rha\%/(Ara $\%+$ Gal\%). The structural characteristics of pectins have been reported previously (Larsen et al., 2018). Statistical analysis was performed in this study, except for DBr. Superscripts (a, b, c, d, and e) within a column show significant differences $(\mathrm{p}<0.05)$ between the pectins, determined by oneway ANOVA, Tukey's post-hoc test. nd: not determined.

Supplementary Table S2: Cumulative production of branched chain fatty acids (BCFA) iso-butyrate and iso-valerate after $72 \mathrm{~h}$ fermentation of pectins and SIEM in TIM-2 colon model 1

\begin{tabular}{llll}
\hline $\begin{array}{l}\text { Pectins } \\
\text { ID }\end{array}$ & $\begin{array}{l}\text { iso-butyrate, } \\
\text { mmol }\end{array}$ & $\begin{array}{l}\text { iso-valerate, } \\
\text { mmol }\end{array}$ & $\begin{array}{l}\text { Total BCFA, } \\
\text { mmol }\end{array}$ \\
\hline P1 & $5.1 \pm 0.8$ & $17.9 \pm 3.1$ & $23.1 \pm 3.9$ \\
P2 & $4.0 \pm 0.6$ & $15.4 \pm 0.8$ & $19.4 \pm 0.2$ \\
P3 & $5.0 \pm 0.9$ & $14.7 \pm 0.5$ & $19.6 \pm 1.4$ \\
P4 & $5.1 \pm 0.7$ & $13.1 \pm 0.6$ & $18.2 \pm 1.3$ \\
P5 & $5.0 \pm 0.1$ & $14.4 \pm 0.6$ & $19.4 \pm 0.7$ \\
P6 & $4.7 \pm 0.8$ & $14.2 \pm 0.4$ & $18.9 \pm 1.2$ \\
P7 & $4.8 \pm 0.3$ & $15.9 \pm 0.8$ & $20.7 \pm 1.2$ \\
P8 & $4.8 \pm 0.5$ & $15.4 \pm 2.3$ & $20.2 \pm 2.8$ \\
P9 & $4.0 \pm 0.3$ & $13.8 \pm 1.9$ & $17.8 \pm 2.2$ \\
P10 & $2.9 \pm 0.9$ & $9.4 \pm 1.4$ & $12.3 \pm 1.9$ \\
SIEM & $3.0 \pm 0.5$ & $18.2 \pm 4.4$ & $21.3 \pm 4.8$ \\
\hline P
\end{tabular}

${ }^{1}$ Mean values $( \pm$ SD) in lumen and dialysate samples (combined) from two independent TIM-2 experiments. Total BCFA is a sum of iso-butyrate and iso-valerate. Pectin codes 
are explained in Table 1. The amount of BCFA at the start of fermentations has been set to zero. SIEM is the basal simulated ileal efflux medium used in control experiments.

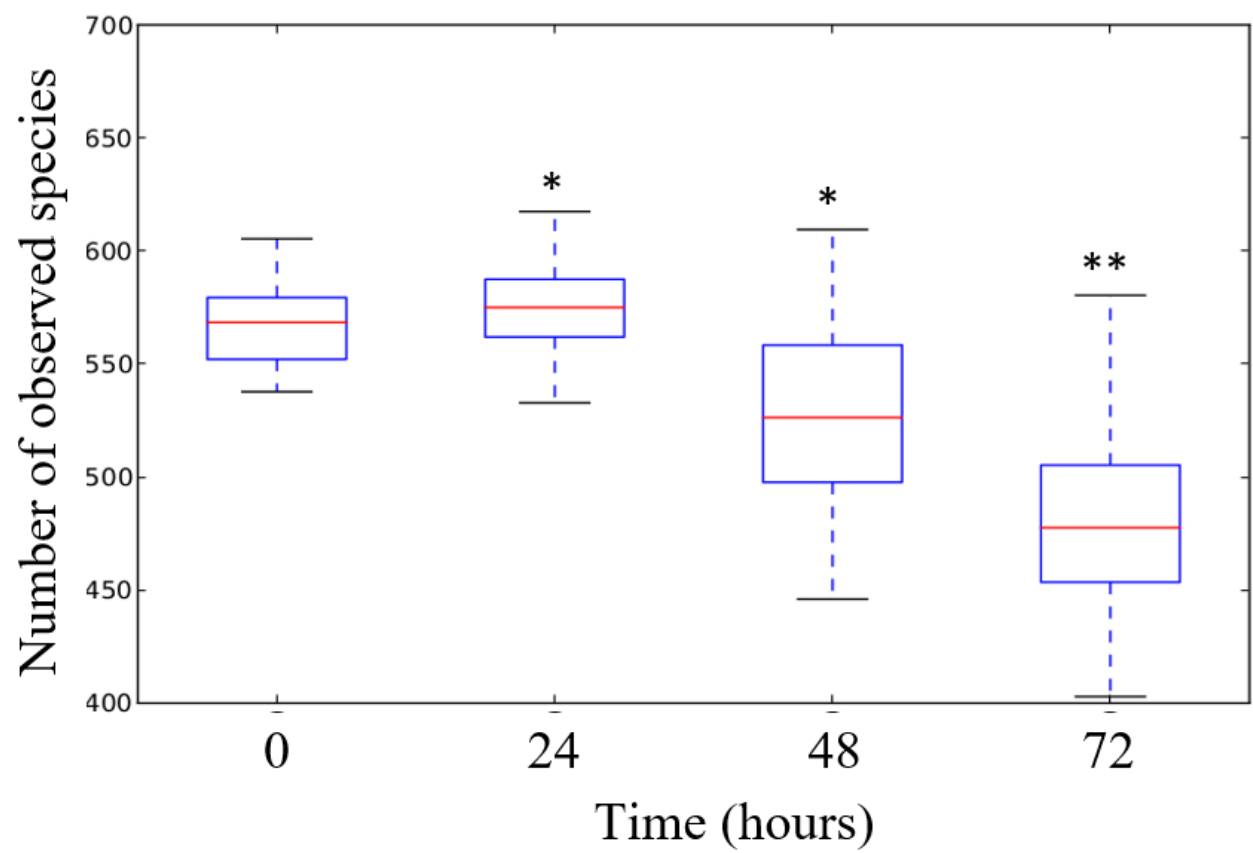

Figure S1: Boxplots show changes in the number of observed species in TIM-2 fermentations of pectins (combined samples) over time: baseline $(0 \mathrm{~h})$ and after 24,48 and $72 \mathrm{~h}$ (center line, median; box limits, first and third quartiles; whiskers, $1.5 \times$ interquartile range). Asterisks denote significant differences compared to baseline (nonparametric Student's t-test employing Monte Carlo method, ${ }^{*} \mathrm{p}<0.05$, ${ }^{* *} \mathrm{p}<0.01$ ). 
A

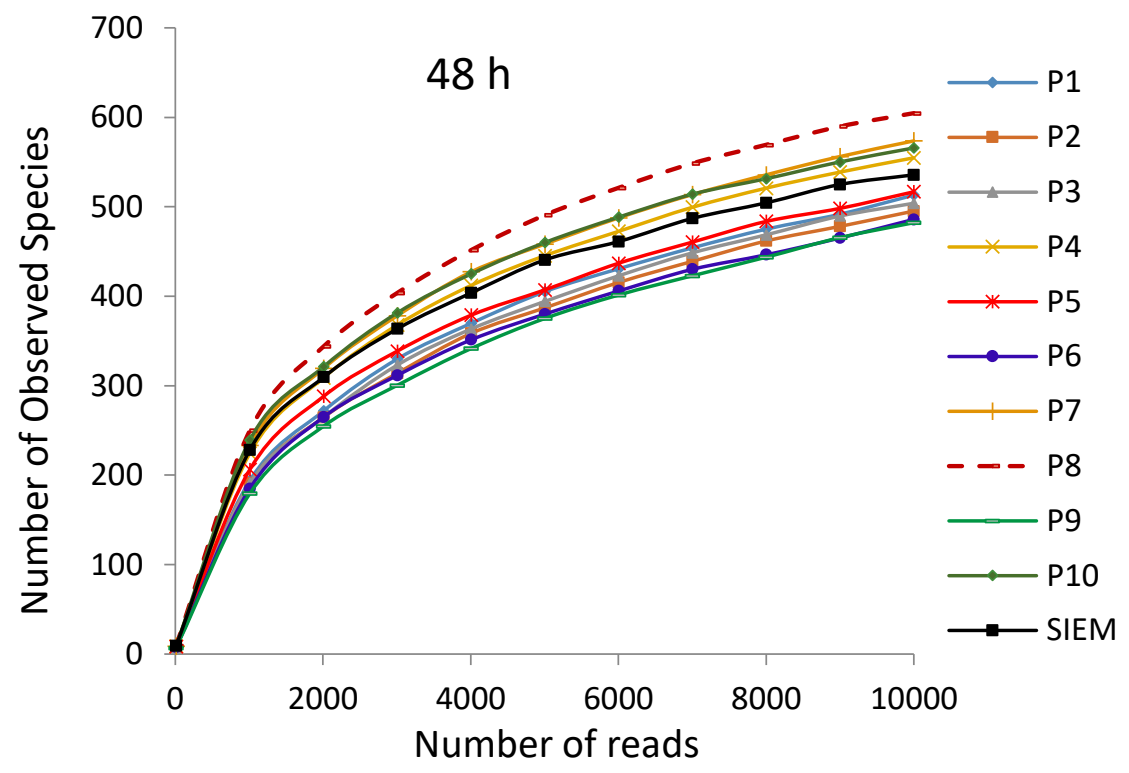

B

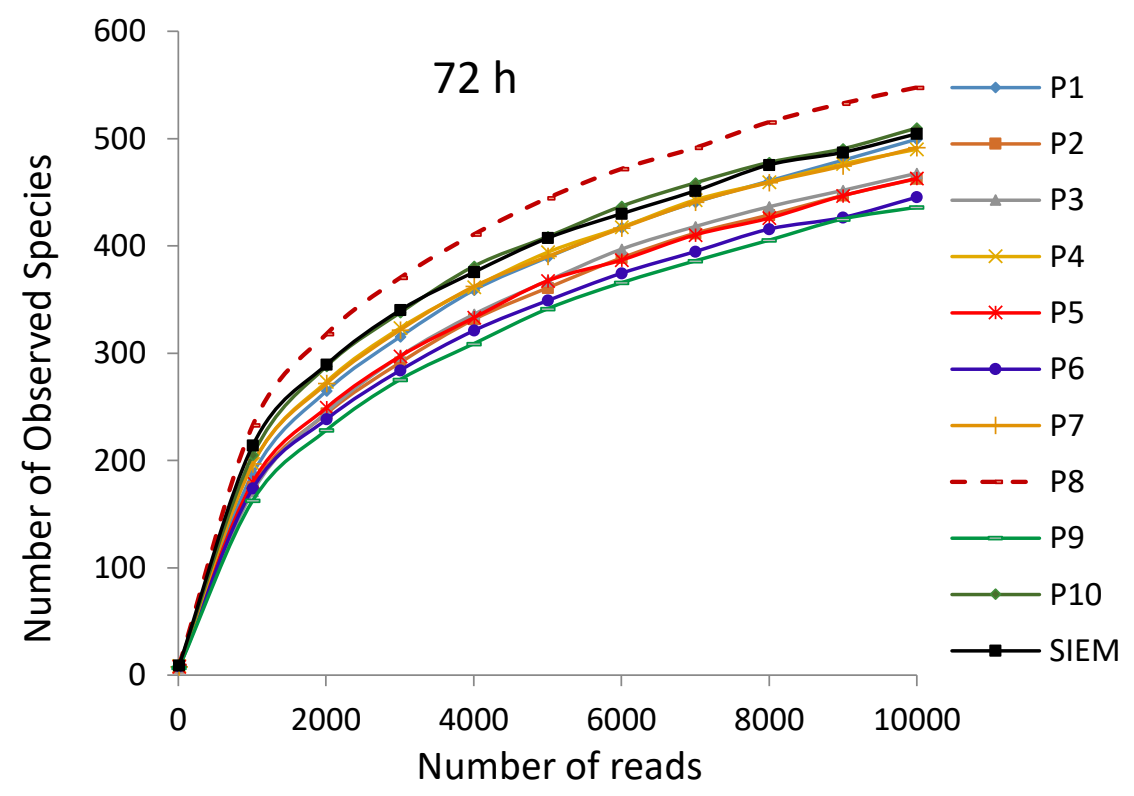

Figure S2: Rarefaction curves showing the number of observed species after $48 \mathrm{~h}(\mathrm{~A})$ and $72 \mathrm{~h}(\mathrm{~B})$ fermentation of pectin (P1 - P9) and RGI (P10) by fecal microbiota in TIM-2 colon model. Microbiota composition was analyzed using NextSeq Illumina DNA amplicon sequencing (V3 region of 16S rRNA gene). Rarefaction curves were generated from the rarefied OTU tables (10.000 reads per sample) as shown by the mean values $( \pm S D)$ from two independent TIM-2 experiments. 


\section{Chapter 8}

General discussion 


\section{Motivation and aim of the research}

The research described in this $\mathrm{PhD}$ thesis was part of the program entitled "Science without borders" launched in 2011 by the Brazilian government. The objective of this program was to promote the consolidation, expansion, innovation and competitiveness of Brazilian science and technology through exchange and international mobility of Brazilian students and researchers to the best universities around the globe. Through international collaboration, the program granted scientific projects in areas considered essential for the development and growth of Brazil. The research carried out and presented in this thesis fitted in the area of "biology, biomedical sciences and health" from the program. Of note, the international collaborations should enable Brazilian researchers to receive knowledge from the top universities to be applied thereafter in Brazil. In turn, this can be translated into self-sufficiency and economic growth, with the main and final goal of promoting a positive impact on the quality of life of the Brazilian population.

Modern society faces a number of challenges that are life-threatening if not taken care of. These range from global warming, with devastating effects on the climate and sea level, to undernutrition in parts of the world and overnutrition associated with a range of health issues in other parts of the world. Moreover, with the growing world population, it becomes increasingly difficult to feed all of those mouths, certainly in a sustainable manner. There is increased pressure on use of land, which must be used for both agriculture and building. Essentially this means that agriculture should be performed as efficient as possible, to allow maximum yield, at the same time keeping food quality and food safety in mind. Currently, it is estimated that $30 \%$ of food production is wasted somewhere along the food chain. This could be due to e.g. $i$ ) loss of biomass in food-processing, where waste is thrown away, ii) disposal of food products that have passed the expiration date, iii) food spoilage, or iv) not consuming all the food that is prepared by consumers at home. If this could largely be prevented, at least for the near future part of the problem of food availability for the growing world population could be solved.

Paradoxically, currently a large part of the world deals with overconsumption, with associated so-called "Western diseases", such as obesity, hypertension, type II diabetes (T2D) and cardiovascular disease (CVD). Taking obesity as an example, overconsumption together with a sedentary life-style in modern society leads to a dysbalance in energy expenditure, causing weight gain. Apart from food intake and energy expenditure however, the gut microbiota has also been shown to play an important role in obesity (1). The trillions of microbes 
that live inside our gut interact with us (the host) at numerous levels, including the immune system, metabolism and even the brain (2). It goes beyond the scope of this discussion to review the knowledge on the role of the microbiota in obesity. For this I refer to Chapter 1 of this thesis.

The primary aim of this thesis was to address some of the challenges modern society faces by studying the use of food processing waste in the context of modulating the gut microbiota in obesity, such that this would provide a health benefit. In this manner, deaths associated with obesity (and T2D, CVD) would be reduced. At the same time, this is done in a sustainable manner of food use, accompanied by efficient use of resources used to prepare the food, such as land, water, and labour.

The main focus of the present research was to investigate the potential prebiotic effects of by-products (waste) from the Brazilian food processing industry of cassava, orange and passion fruit. Differences in fermentation were assessed when using a microbiota originating from lean or obese individuals, and in addition to the Brazilian food by-products the fibres arabinogalactan and inulin were used. The effects of different types of pectins extracted from other diverse food by-products were also evaluated. The approach used was in vitro fermentation in the validated TIM- 2 model. Raw by-products and their alcohol insoluble solid (AIS) fractions (representing the dietary fibre part) were characterized and used for in vitro fermentation. Gut microbiota compositional changes, metabolite production and characterization of the intermediate degradation products in the chyme were evaluated upon addition of the different substrates. The knowledge gained in this research gives the basis for further research aiming at the valorisation of these by-products and the results showed that the by-products could be used as an alternative tool to improve intestinal health and consequently offer an additional approach to tackle obesity. 


\section{Potential prebiotic effects of food by-products - main findings}

The data presented in this thesis show that the tested food by-products cassava bagasses, orange bagasses and passion fruit peels - are promising candidates to become prebiotics in the future (defined as "a substrate that is selectively utilized by host microorganisms conferring a health benefit" (3)). Although there is an urgent need to solve the problem of by-products generation and their proper utilization, few researchers are focusing on testing the potential prebiotic effects in fibre rich food by-products. In this thesis, we have evaluated eight by-products from Brazilian food industry, and from four samples of orange bagasses and passion fruit peels we also evaluated the isolated fibres (AIS).

The in vitro experiments in TIM-2 showed that in general, the food by-products were used by the gut microbiota, and their fermentation resulted in similar amounts of total cumulative production of the beneficial metabolites - SCFA (acetate, propionate and butyrate) as our standard medium (SIEM) and the positive control (inulin), as shown in Figure 1 (Chapters 3-5).

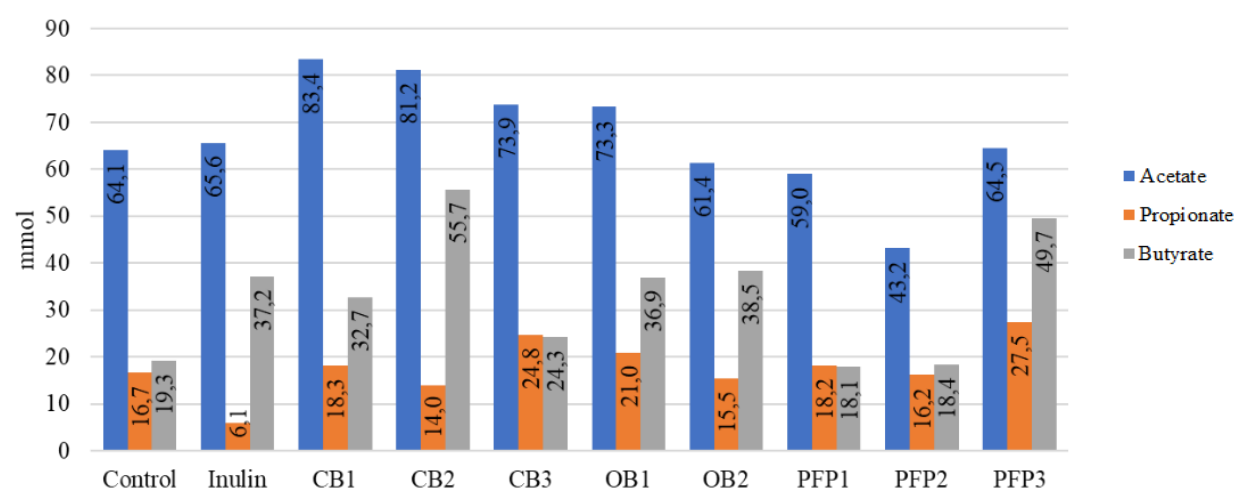

Figure 1: Production of SCFA (acetate, propionate and butyrate) by the obese microbiota after 72 hours fermentation period with different test compounds, control and inulin (positive control) (Chapter 4).

The effects that each by-product had on gut microbiota composition differed according to the type of by-product and the main findings are briefly discussed in the following section.

\section{Cassava bagasses (Chapters 2-4).}

Fermentation of two samples of cassava bagasses in two sets of different experiments were found to stimulate the increase in abundance of the beneficial genus Bifidobacterium (Chapters 3 and 4). 
Few studies have investigated the effects of CB on the gut microbiota. A study performed with the isolated fibre from cassava (not cassava bagasse) was used in an in vitro experiment with human faecal inoculum, and though only SCFA were measured, cassava was found to stimulate the production of butyrate (4). An experiment in rats showed an increase in Lactobacillus counts and reduction of Escherichia coli in animals that received cassava fibre-based diet (5).

Although our results were not similar to those found in the literature, still our studies with CBs resulted in promising potential prebiotic effects, demonstrating that CBs could be further explored in the context of intestinal health.

\section{Orange bagasses (Chapters 4 and 5 ).}

The main findings about gut bacterial fermentation of orange bagasses were that despite OB1 and OB2 having similar chemical composition (Chapter 2), their fermentation profile differed regarding the production of SCFA and the effects that they had on the composition of the microbiota (Chapter 4). At first, our hypothesis was that even small differences in chemical composition may had an divergent effect on the gut microbiota. However, in a different set of experiments with the isolated fibre (AIS) from OBs (A-OB1 and A-OB2) (Chapter 5), it was very interesting to observe that also the chemical composition between the AIS fractions from the two tested OB was very similar, and still, A-OB1and A-OB2 had a distinct fermentation profiles, even more different than the results found with the untreated fibres (Chapter 4). This difference can be explained by solubility. Importantly, this difference in polysaccharide utilization was revealed only with the analysis of intermediate degradation products during fermentation (Chapter 5), something that cannot be investigated in a human clinical trial. Degradation of insoluble fibres is challenging for gut microbes because it requires the adhesion of these bacteria to the cell wall polysaccharides which have lower accessible surface area and hydrogen-bonding networks that holds the carbohydrate chains together $(6,7)$. This difference in the proportion of soluble to insoluble carbohydrates between A-OB1 and A-OB2 likely explains the differences found in the types of microorganisms enriched by each by-product (Chapter 5).

Overall, OBs were found to positively affect the gut microbiota by stimulating an increase of beneficial bacteria, such as Collinsella, Lachnospira, Prevotella and Faecalibacterium (Chapters 4 and 5). 


\section{Passion fruit peels (Chapters 4 and 5).}

Passion fruit peels (PFPs) and their AIS fractions showed the poorest fermentability, based on the smaller amounts of total SCFA.

PFPs also generated negligible amounts of lactate and succinate, indicating that these by-products were not fermented at a high speed. Lactate and succinate are intermediate metabolites in intestinal microbiology and are usually present in lower amounts because they are consumed by other microbes through crossfeeding that convert them into SCFA, such as propionate and butyrate $(8,9)$. Their accumulation is an indication that a substrate is fermented fast (8). Their absence in the fermentation of PFPs reinforce the indication that PFPs were fermented at a slow rate. This is a very interesting characteristic, because a substrate that is fermented slowly has more chances to reach the distal part of the colon to be fermented there. Most carbohydrates are used almost in their entirety in the proximal colon (10). Proteins are consequently used by microbes in the distal colon, producing toxic metabolites which have direct effects on colon cancer incidence and most of the colon cancer occurs distally $(11,12)$.

PFP fermentation resulted in a microbiota with the highest diversity and evenness, a positive trait regarding host health. Although currently it is still difficult to establish what is considered a "healthy microbiota", richness and diversity are generally used as an indication of gut health due to their inverse association with diverse types of diseases (13).

It is acknowledged that a limitation of our research is that the experiments were accomplished in vitro, and therefore we lack the interaction between the gut microbiota and the host. Of course, clinical trials are the golden standard in studying the effects of food (components) on health. However, in clinical trials it is very difficult to study the total production of microbial metabolites (unless very expensive tracer studies with e.g. ${ }^{13} \mathrm{C}$-isotope labelled substrates are used), let alone study the kinetics of production of these metabolites. Also, to measure intermediate degradation product of fibre fermentation, one needs to use very invasive methods with catheters to samples the proximal colon (where most carbohydrate fermentation takes place). Therefore, we made use of the validated, dynamic, computer-controlled in vitro model of the colon developed by TNO (TIM-2; (14)), which has a number of benefits: a) it allows frequent sampling in time, which allows to study both intermediate fermentation products that are produced (which would be missed if faecal samples would be analysed) and it allows for properly studying kinetics of production of microbial metabolites, which is practically impossible in vivo; b) because the 
model accurately mimics physiological conditions, including removal of microbial metabolites (which would otherwise accumulate and inhibit the microbiota) mechanistic studies can be carried out, allowing for instance to determine structure-function relationships (Chapter 7). Again, similar experiments in vivo are virtually impossible to do. One could argue that animal models could be used, but the microbiota of laboratory animals is very different from that of humans and translation from animal models to humans is an issue, on top of the ethical concerns.

\section{Differences in gut bacterial fermentation of fibres when using microbiota from lean or obese individuals}

The gut microbiota plays an important role in maintaining human health and it has been suggested that it can affect the nutritional metabolism of the host with consequences on energy storage, through several mechanisms.

However, the idea that gut bacteria can contribute to the maintenance of the host body weight is characterized by numerous paradoxes. As an example, it has already been shown that a diet rich in non-digestible fibres decreases body weight, fat mass and the severity of diabetes (15-17). Nonetheless, at the same time it is being argued that the same dietary fibres increase strains of bacteria that are able to digest/ferment them and provide extra energy in the form of SCFA (15), which are used as a fuel by several host tissues. However, the proposed increase in energy extraction in the form of SCFA is not the only way that the gut microbiota may affect body weight, but the gut microbiota is also involved, for instance, in gut permeability, metabolic endotoxemia and bile acids metabolism, which have a role in energy metabolism and consequently obesity (18).

In general, we have found differences in fermentation of fibres when using microbiota from lean or obese individuals. The differences were encountered either on the level of microbiota composition or production of metabolites. Interestingly, the production of energy in the form of SCFA was not always higher when fermentation was performed using the obese microbiota, as expected. Mostly, the production of energy was dependent of the type of fibre being used, and not the type of microbiota.

Fermentation of two samples of CBs showed a bifidogenic effect especially in the obese microbiota (Chapters $\mathbf{3}$ and 4). In the context of obesity, a prebiotic which can stimulate the genus Bifidobacterium may have an important role to ameliorate weight gain(ed). A clinical trial with overweight/obese children 
showed that compared to the placebo group, the consumption of prebiotic (oligofructose-enriched inulin) increased Bifidobacterium and was accompanied by a parallel decrease in body weight and percentage of body fat (19). Another study which compared the gut microbiota from healthy and obese children and adolescents showed that there was an increase in Bifidobacterium during the process of weight reduction (20). Additionally, Bifidobacterium was associated with improved gut mucosal barrier and lower intestinal levels of lipopolysaccharide (LPS), and was found in lower abundance in obese subjects (21). Regarding the production of SCFA, CBs stimulated higher production of acetate by the obese microbiota when compared to the lean microbiota. The role of acetate in obesity is not completely understood. On the one hand, a clinical trial with overweight and obese men showed that infusion of acetate in the distal colon increased fat oxidation and fasting PYY, a positive effect to tackle obesity (22). On the other hand, increased acetate production by an altered microbiota was found to rise ghrelin secretion, causing hyperphagia and obesity in rats fed with high fat diet (23). How this translates to humans (that do not perform hyperphagia) remains to be seen.

We suggest that administration of OBs drove the microbiota of obese individuals towards a healthier profile (Chapters 4 and 5). Fermentation of OB2 by the obese microbiota stimulated the increase in abundance of Faecalibacterium. Faecalibacterium prausnitizii is an anti-inflammatory commensal bacterium and studies showed an inverse correlation between inflammatory bowel disease (IBD) and this species (24-26). Stimulation of $F$. prausnitzii may be beneficial in the context of obesity, since this disease is characterized by low-grade inflammation (27). Compared to control, fermentation of OBs by the obese microbiota produced smaller amounts of succinate. Elevated levels of plasma succinate were found to be associated with obesity and impaired glucose metabolism (28).

PFP fermentations stimulated the enrichment Ruminococcus only in the lean microbiota, not in the obese microbiota. Ruminococcus species are known to use recalcitrant fibres for energy extraction, and a possible explanation is that the obese microbiota was not used to have this type of substrate and therefore does not have: $i$ ) the necessary species to degrade efficiently complex fibres and/or ii) the machinery to break down the substrate and make complete use of it (Chapter 4).

Compared to the lean microbiota, fermentation of arabinogalactan by the obese microbiota enriched the abundance of Lactobacillus, Dorea, Faecalibacterium 
and Blautia, showing that this fibre had a positive effect on the obese microbiota (Chapter 6). A study showed a parallel reduction of body mass index (BMI) and increase of Lactobacillus spp. amounts in obese adolescents suggesting a potential role of this genus in obesity and body weight control (29).

Inulin fermentation by the obese microbiota showed an increase in the beneficial genera Bifidobacterium, Lactobacillus, Faecalibacterium and Blautia, when compared to the lean microbiota. A high abundance of bifidobacteria could be protective in obesity since it is speculated that this genus may decrease pro-inflammatory cytokines and decrease metabolic endotoxemia $(30,31)$.

It remains to be verified what the mechanisms are behind the differences found in fermentation of diverse fibres when using lean or obese microbiotas. The hypothesis or logical explanation is that the initial difference in microbiota composition had an effect on the dynamics of fermentation, and that therefore the microbiotas responded in a different manner to feeding of the different fibres. The degree of the microbiota plasticity and their capacity to respond to dietary interventions may be dependent on the baseline population (13). Still, although in different ways, the tested fibres showed positive effects on the microbiota from obese individuals in different studies, demonstrating that these by-products could be a promising tool to assist in the treatment of obesity. And this is very important, especially because currently, therapeutic interventions available to prevent or even reverse the problem of obesity has a limited impact (21), showing the urgent need for new approaches to tackle obesity. However, it is important to keep in mind that obesity is a multifactorial disease, and consequently, most probably a multifactorial approach is necessary to counteract it. In this sense, the manipulation of the gut microbiota into a healthier profile may be one factor, among others, to be used in this battle, and may not represent a "miraculous" unique solution against obesity.

\section{The relationship between fibre structure and gut microbiota fermentation}

Fibres constitute a heterogeneous group of compounds, and consequently the consumption of different types of fibres results into different health effects. This heterogeneity may explain the discrepancy found in studies about the role of fibres in human health, and the effects on the gut microbiota. Therefore, fibre characterization is crucial regarding, for instance, the health effect that is 
desired upon consumption, eventually allowing a personalized nutrition focused on a specific benefit.

To gain a deep understanding of the chemical and physiological characteristics of the by-products selected, we employed a study of chemical characterization and in vitro digestibility (Chapter 2). Although it is common sense that the peels, bagasses and seeds are rich in fibres, our objective was to specify the major type of fibre that each by-product was composed of, and the monosaccharides that were present.

Characterization revealed chemical differences in the three samples of CBs. CB3 was richer in pectin, cellulose and hemicellulose than the other two cassava bagasses tested, and caused a different effect on microbiota composition and production of metabolites. As previously mentioned, CB1 and CB2, richer in starch than CB3, had a positive effect on Bifidobacterium abundance, which was not seem upon CB3 fermentation (Chapter 4). Bifidobacterium mostly ferments mono- and oligosaccharides, but some species degrade complex carbohydrates like starch (32), producing mainly acetate and lactate (33), which may explain its increase upon the CBs that had higher amounts of starch in their composition.

We have found that the genus Ruminococcus was inversely associated with small sugars, and fermentation of OBs in both microbiotas (lean and obese) caused a decrease in this genus. An opposite result was found in an in vitro study performed with lemon pectin and microbiota from obese individuals (34), which enriched the abundance of Ruminococcaceae family. The substrate used in our study was the whole material originating from the manufacturing of orange juice (the whole bagasse), composed not only of pectin, but also small sugars for instance, which likely affected the bacterial dynamics, explaining the discrepancy found. However, when we tested the isolated fibre of OBs (AIS), in the PCoA analysis, A-OB2 clustering was mainly driven by Ruminococcus (Chapter 5). Isolation of fibres via AIS removed the small sugars present in the raw material, and may have stimulated the enrichment of Ruminococcus, since only fibres (mainly pectin and hemi(cellulose)) were present. Some species of Ruminococcus have the ability to ferment recalcitrant fibres in the colon (35), and therefore may have a preference for such substrates. It was demonstrated that species from the genus Ruminococcus, present in the human gut bacterial community had the ability to degrade cellulose (36). Degradation of insoluble fibres is challenging for gut microbes because it requires the adhesion of these bacteria to the cell wall polysaccharides which have lower accessible surface area and hydrogen-bonding networks that holds the carbohydrate chains together $(6,7)$. 
In Chapter 7 we investigated how the structure of nine structurally diverse pectins and the pectic derivative rhamnogalacturonan I (RGI) influenced the dynamics of composition and metabolic activity of the gut microbiota.

Despite differences in the structure of the various citrus pectins tested, the levels of total SCFA produced were not significantly different. This may be due to functional redundancy of microbiota and cross-feeding mechanisms of microbial interactions (37).

Regarding the modulation of the microbiota composition, the major finding in this study was that the changes of specific bacterial taxa were related to structural features of pectins. The main factors that were associated with the shifts in the microbiota composition were the degree of esterification and content and composition of monosaccharides (Chapter 7).

Our results suggested that stimulation of Faecalibacterium prausnitzii can be achieved via fermentation of high methoxylated pectins, rather than low methoxylated pectins.

The data presented in this thesis contribute to elucidate some interactions between the gut microbiota and the structural features of fibres. Due to the inherent complexity of fibres, their structural characterization is crucial to understand how fibre structures may favour individual microbial groups. And in the future, this may be translated into how specific fibre structures or a mix of fibres can be used to maintain a healthy gut microbiota or even how this may be used to transform a dysbiotic gut bacterial population back to a healthy microbiota (38). 


\section{Future perspectives and concluding remarks}

Plant food processing generates by-products in enormous amounts. These byproducts can represent a problem, since their inappropriate disposal is a source of pollution (39). At the same time, consumers are becoming more aware not only about the relationship between diet and health (40), but also regarding the ways of production of their foods: for instance, if the product was manufactured in a sustainable manner. Thus, conscious consumers give preference to natural ingredients produced in a sustainable way, with low or minimal impact on the environment and that are respectful to the labour involved. Bearing in mind such considerations, the use of food by-products represents a very promising field to be explored. They contain valuable compounds, which may be used as functional foods and even replace synthetic additives, constituting a natural alternative source of a vast range of ingredients/compounds (39).

Therefore, our studies were focused on the use of by-products, an innovative approach especially with regard to their effects on the gut microbiota, as demonstrated by few other scientific publications available with these byproducts - cassava bagasse, orange bagasse and passion fruit peel. These waste products high in dietary fibres could be used with the main objective to serve as source of energy for beneficial bacteria residing in the (distal) colon, and through beneficial modulation of the gut microbiota protect the host against health problems that commonly affect our Western society.

Currently, the majority of research on dietary fibres and their effects on gut microbiota focuses on purified ingredients extracted from plants. This, however, is being challenged in terms of human nutrition, because evidence shows that it is the actual complexity of dietary fibres that has an effect on the complexity of the gut microbiota (41). Our results showed that besides the differences found in fermentation by microbiotas from lean and obese individuals, fermentation of the raw by-products and their AIS fractions also differently affected the metabolism and composition of the gut microbiota. These differences could have been caused by many components still present in the whole by-products, such as polyphenols (not measure here) or small sugars for instance, in the composition of OBs. Therefore, the characterization of these by-products was crucial in order to explain the results observed. A limitation of our studies was the small number of replicates, which could have hindered many correlations between specific components with microbes and/or metabolites. 
Another crucial point in our research, which should be adopted by other researchers, was the assessment of the degradation products generated during fermentation. Without this specific analysis, we would not have been able to understand the reasons why two chemically similar fibres (the two OBs) resulted in different fermentation profiles regarding the amounts of SCFAs produced, but more importantly, about the diverse types of microorganisms that were enriched/inhibited on each OB. Although further experiments are necessary to reinforce our hypothesis, the analysis of degradation products during fermentation allowed us to see how differences in carbohydrate solubility affected the dynamics of the gut microbiota, which was observed and described by others $(42,43)$.

The results found in our studies demonstrated the potential of the diverse food by-products in modulating the gut microbiota from lean, obese and healthy individuals. As shown in this thesis, validated, predictive, dynamic, and multicompartmental in vitro models offer an excellent alternative to clinical trials to screen novel foods. Therefore, the next steps could be to test other food byproducts in vitro. Direct comparative studies with the raw food by-product and their isolated fibres are suggested, including the analysis of degradation products formed during fermentation.

For instance, further comparative studies are suggested both in vitro and in vivo testing the effects on the gut microbiota of cassava bagasse from various regions of Brazil, as well as CBs from artisanal and industrial processing. Our in vitro study about the digestibility of CBs demonstrated that around $12 \%$ of the starch present in the samples was resistant starch (RS). Therefore, we suggest that future research with CBs evaluate the amount and type of RS present, since studies have demonstrated that the effects of RS on the gut microbiota varies according to the type of RS administered $(44,45)$. Future studies including predigested cassava bagasse are suggested to explore the effects on the bacterial community and/or metabolism. Monitoring the degradation of carbohydrates during fermentation would contribute to elucidation of the interactions between gut microbiota and the utilization of complex polysaccharides.

Moreover, future studies aiming to assess bacterial utilization of sugars during fermentation with separate soluble and insoluble fractions (e.g. of orange bagasses) are suggested to evaluate the importance of solubility on gut microbiota modulation, preferably in the distal colon.

In addition, in order to confirm our results and to contribute to the important understanding of structure-function relationship of dietary fibres/prebiotics in the gut, further comparative in vitro and in vivo studies with structurally diverse carbohydrate polymers and their derivatives are suggested. 
This thesis raises the awareness of the importance of the research performed with food by-products, and therefore paves the path for further research on the incorporation of by-products into food products, or their use per se, perhaps as a functional food. Their utilization would definitively have an impact on decreasing food wastage (46), as well as bring health benefits for those who consume it. 


\section{References}

1. Backhed F, Ding H, Wang T, Hooper L V, Koh GY, Nagy A, et al. The gut microbiota as an environmental factor that regulates fat storage. Proc Natl Acad Sci U S A. 2004 Nov;101(44):15718-23.

2. Cani PD. Human gut microbiome: hopes, threats and promises. Gut; 2018;67:1716-1725.

3. Gibson GR, Hutkins R, Sanders ME, Prescott SL, Reimer RA, Salminen SJ, et al. Expert consensus document: The International Scientific Association for Probiotics and Prebiotics (ISAPP) consensus statement on the definition and scope of prebiotics. Nat Rev Gastroenterol Hepatol. 2017 Aug;14(8):491-502.

4. Mallillin AC, Trinidad TP, Raterta R, Dagbay K, Loyola AS. Dietary fibre and fermentability characteristics of root crops and legumes. Br J Nutr. 2008;100(3):485-8.

5. Osundahunsi OF, Williams AO, Oluwalana IB. Prebiotic effects of cassava fibre as an ingredient in cracker-like products. Food Funct. 2012;3(2):159-63.

6. Cockburn DW, Koropatkin NM. Polysaccharide Degradation by the Intestinal Microbiota and Its Influence on Human Health and Disease. J Mol Biol. 2016 Aug 14;428(16):3230-52.

7. Lynd LR, Weimer PJ, van Zyl WH, Pretorius IS. Microbial cellulose utilization: fundamentals and biotechnology. Microbiol Mol Biol Rev. 2002 Sep;66(3):506-77.

8. Koenen ME, Cruz Rubio JM, Mueller M, Venema K. The effect of agave fructan products on the activity and composition of the microbiota determined in a dynamic in vitro model of the human proximal large intestine. J Funct Foods. 2016;22:201-10.

9. Koh A, De Vadder F, Kovatcheva-Datchary P, Bäckhed F. From dietary fiber to host physiology: short-chain fatty acids as key bacterial metabolites. Cell. 2016 Jun 2;165(6):1332-45.

10. den Besten G, van Eunen K, Groen AK, Venema K, Reijngoud DJ, Bakker $\mathrm{BM}$. The role of short-chain fatty acids in the interplay between diet, gut microbiota, and host energy metabolism. J Lipid Res. 2013;54(9):232540.

11. Macfarlane GT, Macfarlane S. Bacteria, colonic fermentation, and gastrointestinal health. J AOAC Int. 2012;95(1):50-60.

12. Ou J, DeLany JP, Zhang M, Sharma S, O’Keefe SJD. Association between low colonic short-chain fatty acids and high bile acids in high colon cancer risk populations. Nutr Cancer. 2012;64(1):34-40.

13. Gentile CL, Weir TL. The gut microbiota at the intersection of diet and human health. Science. 2018 Nov;362(6416):776-80.

14. Minekus M, Smeets-Peeters M, Bernalier A, Marol-Bonnin S, Havenaar R, Marteau P, et al. A computer-controlled system to simulate conditions of the large intestine with peristaltic mixing, water absorption and absorption of fermentation products. Appl Microbiol Biotechnol. 
1999;53(1):108-14.

15. Cani PD, Delzenne NM, Amar J, Burcelin R. Role of gut microflora in the development of obesity and insulin resistance following high-fat diet feeding. Pathol Biol (Paris). 2008 Jul;56(5):305-9.

16. Fleissner CK, Huebel N, Abd El-Bary MM, Loh G, Klaus S, Blaut M. Absence of intestinal microbiota does not protect mice from dietinduced obesity. Br J Nutr. 2010;104(6):919-29.

17. Tuohy KM, Rouzaud GC, Bruck WM, Gibson GR. Modulation of the human gut microflora towards improved health using prebiotics--assessment of efficacy. Curr Pharm Des. 2005;11(1):75-90.

18. Cornejo-Pareja I, Munoz-Garach A, Clemente-Postigo M, Tinahones FJ. Importance of gut microbiota in obesity. Eur J Clin Nutr. 2018 Nov;

19. Nicolucci AC, Hume MP, Martinez I, Mayengbam S, Walter J, Reimer RA. Prebiotics reduce body fat and alter intestinal microbiota in children who are overweight or with obesity. Gastroenterology. 2017 Sep;153(3):711-22.

20. Hou Y-P, He Q-Q, Ouyang H-M, Peng H-S, Wang Q, Li J, et al. Human gut microbiota associated with obesity in chinese children and adolescents. Biomed Res Int. 2017;2017:7585989.

21. Lee P, Yacyshyn BR, Yacyshyn MB. Gut microbiota and obesity: An opportunity to alter obesity through faecal microbiota transplant (FMT). Diabetes, Obes Metab [Internet]. 2018 Oct 17;0(0).

22. van der Beek CM, Canfora EE, Lenaerts K, Troost FJ, Damink SWMO, Holst JJ, et al. Distal, not proximal, colonic acetate infusions promote fat oxidation and improve metabolic markers in overweight/obese men. Clin Sci (Lond). 2016 Nov;130(22):2073-82.

23. Perry RJ, Peng L, Barry NA, Cline GW, Zhang D, Cardone RL, et al. Acetate mediates a microbiome-brain- $\beta$ cell axis promoting metabolic syndrome. Nature. 2016 Jun 9;534(7606):213-7.

24. Miquel S, Martín R, Rossi O, Bermúdez-Humarán LG, Chatel JM, Sokol H, et al. Faecalibacterium prausnitzii and human intestinal health. Curr Opin Microbiol. 2013;16(3):255-61.

25. Machiels K, Joossens M, Sabino J, De Preter V, Arijs I, Eeckhaut V, et al. A decrease of the butyrate-producing species Roseburia hominis and Faecalibacterium prausnitzii defines dysbiosis in patients with ulcerative colitis. Gut. 2014 Aug;63(8):1275-83.

26. Singh RK, Chang H-W, Yan D, Lee KM, Ucmak D, Wong K, et al. Influence of diet on the gut microbiome and implications for human health. J Transl Med [Internet]. 2017;15(1):73.

27. Cani PD, Amar J, Iglesias MA, Poggi M, Knauf C, Bastelica D, et al. Metabolic endotoxemia initiates obesity and insulin resistance. Diabetes. 2007 Jul;56(7):1761-72.

28. Serena C, Ceperuelo-Mallafre V, Keiran N, Queipo-Ortuno MI, Bernal R, Gomez-Huelgas R, et al. Elevated circulating levels of succinate in human obesity are linked to specific gut microbiota. ISME J. 2018 
Jun;12(7):1642-57.

29. Santacruz A, Marcos A, Warnberg J, Marti A, Martin-Matillas M, Campoy $\mathrm{C}$, et al. Interplay between weight loss and gut microbiota composition in overweight adolescents. Obesity (Silver Spring). 2009 Oct;17(10):190615.

30. Sarbini SR, Kolida S, Deaville ER, Gibson GR, Rastall RA. Potential of novel dextran oligosaccharides as prebiotics for obesity management through in vitro experimentation. Br J Nutr. 2014 Oct;112(8):1303-14.

31. Tilg H. Obesity, metabolic syndrome, and microbiota: multiple interactions. J Clin Gastroenterol. 2010 Sep;44 Suppl 1:S16-8.

32. Rajilić-Stojanović M, de Vos WM. The first 1000 cultured species of the human gastrointestinal microbiota. FEMS Microbiol Rev. 2014;38(5):996-1047.

33. De Graaf A, Venema K. Gaining Insight into Microbial Physiology in the Large Intestine: A Special Role for Stable Isotopes. 2007;53:73-314.

34. Bianchi F, Larsen N, de Mello Tieghi T, Adorno MAT, Kot W, Saad SMI, et al. Modulation of gut microbiota from obese individuals by in vitro fermentation of citrus pectin in combination with Bifidobacterium longum BB-46. Appl Microbiol Biotechnol. 2018 Aug;

35. Flint HJ, Duncan SH, Louis P. The impact of nutrition on intestinal bacterial communities. Curr Opin Microbiol. 2017 Aug;38:59-65.

36. Chassard C, Delmas E, Robert C, Lawson PA, Bernalier-Donadille A. Ruminococcus champanellensis sp. nov., a cellulose-degrading bacterium from human gut microbiota. Int J Syst Evol Microbiol. 2012 Jan;62(Pt 1):138-43.

37. Reichardt N, Vollmer M, Holtrop G, Farquharson FM, Wefers D, Bunzel M, et al. Specific substrate-driven changes in human faecal microbiota composition contrast with functional redundancy in short-chain fatty acid production. ISME J. 2018 Feb;12(2):610-22.

38. Hamaker BR, Tuncil YE. A perspective on the complexity of dietary fiber structures and their potential effect on the gut microbiota. J Mol Biol. 2014 Nov 25;426(23):3838-50.

39. Schieber A. Side streams of plant food processing as a source of valuable compounds: selected examples. Annu Rev Food Sci Technol. 2017 Feb;8:97-112.

40. Schieber A, Stintzing FC, Carle R. By-products of plant food processing as a source of functional compounds-recent developments. Trends Food Sci Technol. 2001;12(11):401-13.

41. Williams BA, Grant LJ, Gidley MJ, Mikkelsen D. Gut fermentation of dietary fibres: physico-chemistry of plant cell walls and implications for health. Int J Mol Sci. 2017 Oct;18(10).

42. Ramasamy US, Venema K, Schols HA, Gruppen H. Effect of soluble and insoluble fibers within the in vitro fermentation of chicory root pulp by human gut bacteria. J Agric Food Chem. 2014;62(28):6794-802.

43. Gong L, Cao W, Chi H, Wang J, Zhang H, Liu J, et al. Whole cereal grains 
and potential health effects: Involvement of the gut microbiota. Food Res Int. 2018;103:84-102.

44. Martinez I, Kim J, Duffy PR, Schlegel VL, Walter J. Resistant starches types 2 and 4 have differential effects on the composition of the fecal microbiota in human subjects. PLoS One. 2010 Nov;5(11):e15046.

45. Salonen A, Lahti L, Salojarvi J, Holtrop G, Korpela K, Duncan SH, et al. Impact of diet and individual variation on intestinal microbiota composition and fermentation products in obese men. ISME J. 2014 Nov;8(11):2218-30.

46. Sharma SK, Bansal S, Mangal M, Dixit AK, Gupta RK, Mangal AK. Utilization of Food Processing By-products as Dietary, Functional, and Novel Fiber: A Review. Crit Rev Food Sci Nutr. 2016 Jul 26;56(10):164761. 


\section{Summary}




\section{Summary}

The industrial processing of foods is responsible for generating enormous amounts of by-products that are underused and/or discarded. If inappropriately disposed off, they are a source of pollution. There is, therefore, an urgent need to find a sustainable solution to deal with food by-products. In the case of plant byproducts, such as bagasses and peels, because of their fibre content, they may be reintroduced into food products as functional ingredients - aiming to improve intestinal health and energy metabolism via the gut microbiota.

In Chapter 1 an overview is provided about the generation of food by-products and the paradox among food wastage, undernourishment and obesity. Brazil, as the largest producer of orange and passion fruit juices, and an important producer and consumer of cassava, generates considerable amounts of byproducts originating from these processing. Cassava bagasse, orange bagasse and passion fruit peel are described.

The gut microbiota has emerged as an important environmental factor that can affect host metabolism, which can be modulated via diet - especially dietary fibres. A brief description of gastrointestinal tract functioning, gut microbiota and dietary fibres is given.

The problem of obesity and the influence of the gut microbiota on host metabolism is also reviewed in Chapter 1. Additionally, the main approach used in this research - the use of the TNO Intestinal Models (TIM- 1 and 2) is described.

The aim of the present research was to assess the potential prebiotic effects of a number of different (Brazilian) food by-products using an in vitro model of the proximal colon, either with microbiota from healthy individuals or from lean or obese individuals.

First, chemical characterization of eight food by-products (three cassava bagasses [CBs], two orange bagasses [OBs] and three passion fruit peels [PFPs]) and their respective alcohol insoluble solids (AIS) fractions is shown in Chapter 2. Digestibility experiments using the in vitro model TIM-1 with all CBs, one OB and one PFP are also described. Results revealed that for CBs, two samples (CB1 and CB2) were chemically more similar than CB3. CB3 had less starch and more pectin and non-starch polysaccharides (NSP) than the other two samples of CBs. Their AIS fractions were higher in pectin and (hemi)cellulose than the raw byproduct. In vitro digestibility experiments indicated that $\sim 12 \%$ of the starch present in CBs was resistant starch (RS).

Chemical analyses of OBs showed similarity between the two samples. High methylated pectin and small sugars (likely from residual juice) were the main components of OBs. AIS fractions of OBs were also composed of higher amounts 
of pectin than their respective raw by-products. Digestibility experiments showed that $86.3 \%$ of glucose present as starch and small sugar was digestible. Two of the PFP samples (PFP1 and PFP2) had a similar chemical composition, with $\sim 20 \%$ of pectin and $\sim 16 \%$ of NSP (hemi)cellulose. PFP3, however, showed an unusual high amount of starch (38\%), and it is likely that this sample was "contaminated" with starch or another by-product. Approximately $83 \%$ of this starch was digestible. AIS fractions of PFPs had similar pectin amount as the raw by-product.

Differences found in the chemical composition of by-products from different batches, and their AIS fractions may result in divergent fermentation profiles when fermented by the gut microbiota, and therefore such characterization is crucial regarding the use of prebiotics, when aiming for host and intestinal health.

Chapter 3 describes the first set of experiments performed to test in vitro (in TIM-2) the potential prebiotic effect(s) of one sample of cassava bagasse (CB1) when using a microbiota originating from lean or obese individuals (from now on referred to as "lean and obese microbiota"). Overall, CB1 fermentation showed a pronounced bifidogenic effect in both microbiotas but resulted into otherwise different bacterial and metabolite profiles when using lean or obese microbiotas. This first study with CB1 resulted in promising results, and therefore a new set of experiments was performed with CB1 in order to see whether such results could be replicated - especially the bifidogenic effect. Additionally, the interplay between fermentation of different substrates by gut microbiota and host health is not completely understood. Therefore, in Chapter 4 we tested all food by-products characterized in Chapter 2 - three CBs, two OBs and three PFPs, regarding the potential prebiotic effects using the same in vitro approach as in Chapter 3 - TIM-2. Also here, two sets of experiments - using faecal slurry from lean or obese individuals were performed. This chapter describes the effects that each food by-product had on the dynamics of bacterial fermentation. The results from this study showed that not only the two types of microbiota - lean and obese, metabolized the same by-products differently, but also chemically similar substrates, such as the two OBs, resulted in divergent effects on the microbiota composition and end-products generated. This study showed that food by-products have the potential to be used as an alternative tool to improve gut health and consequently host health in the context of obesity.

After experiments with the raw by-product, we wanted to assess whether the isolated fibres from food by-products would exert similar effects on the gut microbiota. Thus, AIS fractions of OBs (A-OB1 and A-OB2) and PFPs (A-PFP1 and A-PFP2) were used in in vitro fermentation experiments using a microbiota from healthy individuals, as described in Chapter 5. Through the analysis of 
intermediate degradation products formed during fermentation we could investigate the bacterial utilization of the different monosaccharides present in these polysaccharides. This study confirmed the observation in Chapter 4, that, although A-OBs were chemically similar, their fermentation profile was different. Only through the analysis of intermediate products formed during fermentation it was possible to observe differences regarding the proportion of soluble to insoluble of each $\mathrm{A}-\mathrm{OB}$, and this difference may have influenced the dynamics of the gut microbiota, explaining the discrepancies found between the two different batches.

Chapter 6 focuses on the differences in fermentation of two fibres - inulin and arabinogalactan, regarding their use by the microbiota from lean or obese individuals. This research showed that metabolism profile was indeed different when fermentation occurred in the lean or obese microbiotas. Importantly, these two fermentable fibres were found to modulate the obese microbiota towards a healthier milieu, reinforcing the fact that the use of prebiotics may represent an inexpensive and non-invasive way to tackle obesity.

The structural complexity of dietary fibre and their influence on the dynamics in changes of the gut microbiota is still not completely known. Therefore, in Chapter 7 how the structure of pectins influenced the dynamics of the gut microbiota was investigated, regarding its potential to modulate composition and/or metabolic activity. Experiments were performed using TIM-2, using microbiota from healthy individuals, and nine structurally diverse pectins plus a pectic derivative rhamnogalacturonan I (RGI). This study helped to elucidate that the effects of pectins in the modulation of the gut microbiota was dependent on their structural features, and emphasized results in earlier chapters, which showed that substrates that are evaluated on their effect on the gut microbiota (composition and activity) should be structurally characterized, to be able to make structure-function relationships.

In Chapter 8, a general discussion of the results is provided, including some ideas for future research. 


\section{Samenvatting}




\section{Samenvatting}

Het industrieel processen van voedsel is verantwoordelijk voor enorme hoeveelheden bijproducten die niet of nauwelijks in de voedingsbereiding gebruikt worden, of zelfs weg gegooid worden. In het laatste geval kunnen ze in sommige gevallen zelfs voor vervuiling van het milieu zorgen. Daarom is er urgent behoefte aan een duurzame oplossing voor het verwerken van voedsel bijproducten. In het geval van plantaardige bijproducten, zoals pulp en schillen, vanwege de aanwezigheid van (voedings)vezels, kunnen deze als functionele ingrediënten opnieuw geïntroduceerd worden in de voedingsketen - met als doel om darmgezondheid en energie metabolisme via de darm microbiota (vroeger darmflora genoemd) te verbeteren.

Hoofdstuk 1 geeft een overzicht over het genereren van voedsel bijproducten en de paradox van voedsel verspilling, ondervoeding en obesitas. Brazilië, de grootste producent van sinaasappel- en passievruchtensappen, en een belangrijke producent en consument van cassave, genereert aanzienlijke hoeveelheden bijproducten die afkomstig zijn van deze verwerking. Cassavepulp, sinaasappel-pulp en passievruchtenschillen worden in dit hoofdstuk beschreven.

De darm microbiota is recentelijk beschouwd als een belangrijke (omgevings)factor die het gastheer metabolisme kan beïnvloeden, en dat kan worden gemoduleerd via het dieet, met name door voedingsvezels. Een korte beschrijving van het functioneren van het maag-darmkanaal, darm microbiota en voedingsvezels wordt gegeven.

Het probleem van obesitas en de invloed van de darm microbiota op het gastheer metabolisme wordt ook besproken in Hoofdstuk 1. Daarnaast wordt de belangrijkste benadering die in dit onderzoek is gebruikt beschreven - het gebruik van de valideerde in vitro modellen van het maag-darm kanaal van TNO (TIM-1 en TIM-2).

Het doel van het huidige onderzoek was om de potentiële prebiotische effecten van een aantal verschillende (Braziliaanse) voedsel bijproducten te beoordelen met behulp van een in vitro model van de proximale colon (TIM-2), hetzij met een microbiota van gezonde individuen, of van obese personen vergeleken met personen met normaal gewicht. Dit staat beschreven in de andere hoofdstukken en wordt hieronder kort samengevat.

Ten eerste wordt de chemische karakterisering van acht voedsel bijproducten (drie monsters van cassave-pulpen [CB's], twee sinaasappel-pulpen [OB's] en drie passievruchtenschillen [PFP's]) en hun respectievelijke in alcoholonoplosbare vaste stoffen (AIS)-fracties beschreven in Hoofdstuk 2 . Verteerbaarheids-experimenten met behulp van het in vitro model TIM-1 met 
alle CB's, één OB en één PFP worden ook beschreven. Uit de resultaten bleek dat voor CB's twee monsters (CB1 en CB2) chemisch meer vergelijkbaar waren dan CB3. CB3 had minder zetmeel en meer pectine en niet-zetmeel-polysacchariden (NSP) dan de andere twee monsters van CB's. Hun in alcohol onoplosbare vaste stoffen (AIS)-fracties waren hoger in pectine en (hemi) cellulose dan het ruwe bijproduct. Deze in vitro verteerbaarheids-experimenten toonden aan dat 12\% van het zetmeel dat aanwezig was in CB's resistent zetmeel (RS) was.

Chemische analyses van de OB's toonden gelijkenis tussen de twee monsters aan. Hoog gemethyleerd pectine en kleine suikers (waarschijnlijk van resterend sap) waren de belangrijkste componenten van OB's. AIS-fracties van OB's waren ook samengesteld uit grotere hoeveelheden pectine dan hun respectieve ruwe bijproducten. Verteerbaarheids-experimenten toonden aan dat $86,3 \%$ van de aanwezige glucose als zetmeel en kleine suiker verteerbaar was.

Twee van de PFP-monsters (PFP1 en PFP2) hadden een vergelijkbare chemische samenstelling, met $\sim 20 \%$ pectine en $\sim 16 \%$ NSP (hemi (cellulose)). PFP3 vertoonde echter een ongewoon hoge hoeveelheid zetmeel (38\%) en het is waarschijnlijk dat dit monster "verontreinigd" was met zetmeel of een ander bijproduct. Ongeveer $83 \%$ van dit zetmeel was verteerbaar. AIS-fracties van PFP's hadden een vergelijkbare pectine-hoeveelheid als het ruwe bijproduct. Verschillen in de chemische samenstelling van bijproducten uit verschillende batches en hun AIS-fracties kunnen resulteren in afwijkende fermentatieprofielen wanneer ze worden gefermenteerd door de darm microbiota, en daarom is een dergelijke karakterisering van cruciaal belang met betrekking tot het gebruik van prebiotica bij het streven naar de gezondheid van de gastheer en de darm.

Hoofdstuk 3 beschrijft de eerste reeks experimenten die werden uitgevoerd om in vitro (in TIM-2) het potentiële prebiotische effect (de effecten) van één monster cassave-pulp (CB1) te testen bij gebruik van een microbiota afkomstig van normaal-gewicht of obese personen (vanaf nu verwezen als "gewoon en obese microbiota"). Over het geheel genomen vertoonde de CB1-fermentatie een uitgesproken bifidogeen effect in beide microbiota's maar resulteerde dit in anderszins verschillende bacteriële en metaboliet-profielen bij het gebruik van gewone of obese microbiota's.

Deze eerste studie met CB1 resulteerde in veelbelovende resultaten en daarom werd een nieuwe reeks experimenten uitgevoerd met CB1 om te zien of dergelijke resultaten konden worden gerepliceerd - met name het bifidogene effect. Bovendien is het samenspel tussen fermentatie van verschillende substraten door de darm microbiota en gastheer-gezondheid niet volledig begrepen. Daarom testten we in Hoofdstuk 4 alle voedsel bijproducten gekarakteriseerd in Hoofdstuk 2 - drie CB's, twee OB's en drie PFP's, met 
betrekking tot de potentiële prebiotische effecten met dezelfde in vitro benadering als in Hoofdstuk 3 - TIM-2. Ook hier werden twee reeksen experimenten uitgevoerd - met behulp van fecale slurry van gewone of obese personen. Dit hoofdstuk beschrijft de effecten die elk voedsel bijproduct had op de dynamiek van bacteriële fermentatie. De resultaten van deze studie toonden aan dat niet alleen de twee soorten microbiota - gewoon en obees - dezelfde bijproducten verschillend metaboliseerden, maar ook dat chemisch vergelijkbare substraten, zoals de twee OB's, resulteerden in uiteenlopende effecten op de samenstelling van de microbiota en eind-producten gegenereerd. Deze studie toonde aan dat voedsel bijproducten het potentieel hebben om te worden gebruikt als een alternatief hulpmiddel om de darmgezondheid en bijgevolg de gezondheid van de gastheer te verbeteren in de context van obesitas.

$\mathrm{Na}$ experimenten met het ruwe bijproduct wilden we beoordelen of de geïsoleerde vezels uit voedsel bijproducten vergelijkbare effecten zouden hebben op de darm microbiota. Aldus werden AIS-fracties van OB's (A-OB1 en AOB2) en PFP's (A-PFP1 en A-PFP2) gebruikt in in vitro fermentatie-experimenten met behulp van een microbiota van gezonde individuen, zoals beschreven in Hoofdstuk 5. Door de analyse van tussentijdse degradatieproducten gevormd tijdens fermentatie konden we het bacteriële verbruik van de verschillende monosacchariden aanwezig in deze polysacchariden onderzoeken. Deze studie bevestigde de observatie in Hoofdstuk 4 dat, hoewel A-OB's chemisch vergelijkbaar waren, hun fermentatieprofiel anders was. Enkel en alleen door de analyse van tussenproducten gevormd tijdens fermentatie was het mogelijk om verschillen te observeren met betrekking tot de verhouding van oplosbaar tot onoplosbaar vezel van elke A-OB, en dit verschil kan de dynamica van de darm microbiota beïnvloed hebben, wat de verschillen gevonden tussen de twee batches waarschijnlijk verklaard.

Hoofdstuk 6 richt zich op de verschillen in fermentatie van twee fermenteerbare vezels - inuline en arabinogalactan, met betrekking tot het gebruik ervan door de microbiota van gewone of obese personen. Dit onderzoek toonde aan dat het metabolisme-profiel inderdaad anders was wanneer fermentatie plaatsvond in de gewone of obese microbiota's. Belangrijk is dat deze twee fermenteerbare vezels de obese microbiota naar een gezonder milieu lieten moduleren, wat het feit versterkt dat het gebruik van prebiotica een goedkope en niet-invasieve manier kan zijn om obesitas aan te pakken.

De structurele complexiteit van voedingsvezels en hun invloed op de dynamiek in veranderingen van de darm microbiota is nog steeds niet volledig bekend. Daarom werd in Hoofdstuk $\mathbf{7}$ onderzocht hoe de structuur van pectines de 
dynamica van de darm microbiota beïnvloedde, met betrekking tot het vermogen ervan om de samenstelling en/of metabolische activiteit te moduleren. Experimenten werden uitgevoerd in TIM-2, met behulp van een microbiota van gezonde individuen, en negen structureel verschillende pectines plus het pectisch derivaat rhamnogalacturonan I (RGI). Deze studie hielp om te verduidelijken dat de effecten van pectines in de modulatie van de darm microbiota afhankelijk waren van hun structurele kenmerken en benadrukte de resultaten in eerdere hoofdstukken, waaruit bleek dat substraten die worden geëvalueerd op hun effect op de darm microbiota (samenstelling en/of activiteit) qua structuur moet worden gekarakteriseerd, om structuur-functie relaties te kunnen maken/achterhalen.

In Hoofdstuk 8 wordt een algemene bespreking van de resultaten gegeven, inclusief enkele ideeën voor toekomstig onderzoek. 


\section{Valorization}




\section{Societal relevance}

Food waste can occur at different levels in the food supply chain, such as during harvesting, storage, processing, packaging and at the consumer's home (1). The term "food by-product" generally refers to edible parts of food that during the processing were discarded, lost, degraded or consumed by pests (1). They are the residual materials from food processing of vegetal (or animal) origin, such as peels, seeds and bagasses (2).

Although food processing technology has brought several positive aspects regarding for instance increasing shelf life of certain food products, the production of ultra-processed foods is linked with several negative effects at distinct levels:

- Food processing is responsible for about $39 \%$ of the total food waste generated around the globe (3);

- Production of highly processed foods generates great amounts of underused by-products that contribute to environmental pollution (4);

- Discarding of food by-products means misuse of natural resources like water and soil (5);

- The consumption of ultra-processed foods has negative effects to human health (6). The production of this type of foods includes the addition of artificial preservatives, bulky agents, artificial flavouring and colourings and the removal of important nutrients, such as fibres (7).

- Consumption of ultra-processed foods is contributing to a major global health problem: obesity (7).

At the same time that food industries discard these food by-products, it was estimated that in 2016, 815 million people suffered of undernutrition (8).

In general, fruit and vegetables by-products, such as peels and bagasses are sources of (amongst others) different dietary fibres, which are suitable for human consumption (9). Giving the circumstances mentioned above, their disposal is unacceptable, and reflects the paradox of the current way of living in our society. In summary, food processing generates huge amounts of food byproducts. Their disposal in the environment is a source of pollution and at the same time, the wastage of food occurs hand in hand with starvation. Paradoxically, the production of ultra-processed foods is leading to a consumption of poor nutrient diets. Consumption of poor nutrient diets have consequences for health, which includes malnourishment, such as obesity (Figure 1), whereas the food-by products contain nutrients that are required for health, such as fibres and anti-oxidants. 

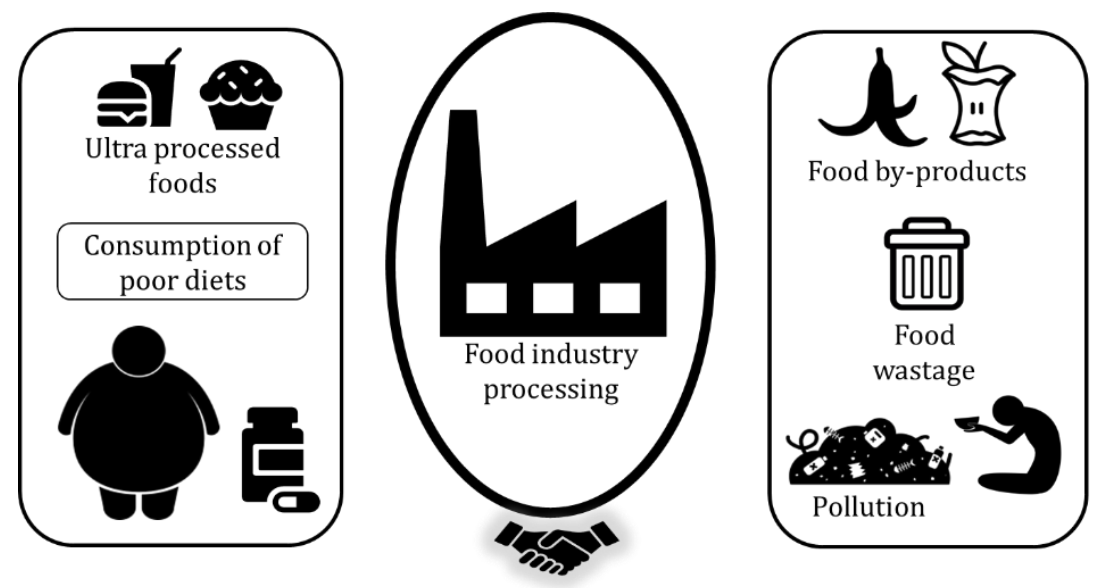

Figure 1: Food products undergo processing, by which the removal of food components generates by-products that are underused - discarded - causing environmental pollution. At the same time, there are people around the world suffering of starvation/undernutrition. The consumption of ultra-processed foods is detrimental to human health, leading to different health problems, including obesity. This is an unfortunate process filled with paradoxes.

There is a necessity to create solutions aiming at a conscious use/destination of food by-products, and scientific research in this field is one way to achieve such demand.

Thus, any attempt to tackle the problem of food by-product underuse is extremely important because it can constitute a beneficial solution that affects both societal health and the environment.

Our work focused on exploring the characteristics of selected Brazilian food byproducts and their potential health benefits by investigating their in vitro effects on the gut microbiota. Food by-products are very diverse and thus have the potential to be used in several food segments. We have focused on Brazilian byproducts generated from starch and flour production of cassava and juice production of oranges and passion fruit, and their beneficial aspect regarding the fibre content and modulation of the gut microbiota in lean and obese people.

The results showed here demonstrate the suitability of the tested by-products in being used as food for humans, and their possible effects related to gut health. Also, our results pave the path for further research on the incorporation of byproducts into food products, or their use per se, perhaps as a functional food. Importantly, consumers are becoming more aware about the consequences their food choices can have, not only regarding the individual aspect, such as health, but also considering the collective or societal effects. Sticking to a sustainable 
diet is perhaps the most crucial step that a responsible consumer can do in their daily lives. This topic is discussed in the next section.

\section{Sustainable diet and sustainable food system}

A sustainable development (of food use) guarantees that the needs of the current generation are achieved without compromising the ability of future generations to achieve their own needs (10). In this context, it is acknowledged that both a healthy diet and a diet that leads to less food wastage reduce the negative impact on the environment, leading to the notion of sustainable diets (11). The term "sustainable diets" was defined by the Food and Agricultural Organization (FAO) as "diets with low environmental impacts, which contribute to food and nutrition security and to healthy life for present and future generations. Sustainable diets are protective and respectful of biodiversity and ecosystems, culturally acceptable, accessible, economically fair and affordable; nutritionally adequate, safe and healthy; while optimising natural and human resources" (12).

\section{"A sustainable food system is a food system that ensures food security and nutrition for all in such a way that the economic, social and environmental bases \\ to generate food security and nutrition of future generations are not compromised" (11)}

The results presented in this thesis contribute to two aspects regarding sustainability. The first relates to sustainable diets. Results shown here demonstrate the potential health benefits that the consumption of food byproducts might bring, especially regarding gut health through the gut microbiota. The by-products tested are natural, rich in fibres and the product of food processing that would otherwise be discarded. Thus, as previously mentioned, the consumption of a healthy diet including these by-products reduces the detrimental impact on the environment. The composition of the diet chosen by consumers has the capacity to drive the demand of specific food products, or specific ways of manufacturing, which has a social, economic and environmental effect (11). In this regard, consumers have the power to push food companies to a sustainable manner of production. For that, information about sustainable diets is crucial.

The second aspect is associated with a sustainable food system. The use of food by-products contributes to a sustainable food system, decreasing the negative 
impact that their disposal would have on the environment. Additionally, utilization of the food product as a whole is a responsible use of natural resources that were employed for plants cultivation, such as soil and water, as well as the human labour that was applied (11).

\section{Target group - overweight and obese individuals}

Obesity is characterized by abnormal or excessive body fat accumulation that might impair an individual's health. In adults, overweight is equivalent to body mass index (BMI) between $25-30 \mathrm{~kg} / \mathrm{m}^{2}$, while obesity is defined by a BMI higher than $30 \mathrm{~kg} / \mathrm{m}^{2}$ (13). Increased BMI is a risk factor for several diseases associated with obesity, such as diabetes, cardiovascular diseases and cancer (14).

Obesity has almost tripled since 1975 around the globe, and in 2016, almost 2 billion adults were overweight, while 650 million were obese (14). There are many factors that contribute to the onset of overweight and obesity, such as bad eating habits essentially due to increased intake of ultra-processed foods which are dense in energy and poor in nutrients (empty calories), and physical inactivity (13). However, it has been hypothesized that these factors cannot solely explain the pandemic of obesity. It has been suggested that a disturbed gut microbiota (dysbiosis) can have a role in obesity $(15,16)$.

Modern life style is causing changes in the gut microbiota (Figure 2) and may explain not only the pandemic of obesity, but also the increased incidence in others diseases, such as asthma, inflammatory bowel disease, autism, food allergies and many others (17).

Although the role of the gut microbiota on host metabolism and obesity is not completely understood, a balanced diet, which includes the consumption of fibres and/or prebiotics, can have a positive effect on the gut microbiota, and consequently on health.

Our results have shown that the use of food by-products can lead to a healthier profile of the gut microbiota, especially the microbiota originating from obese individuals. 


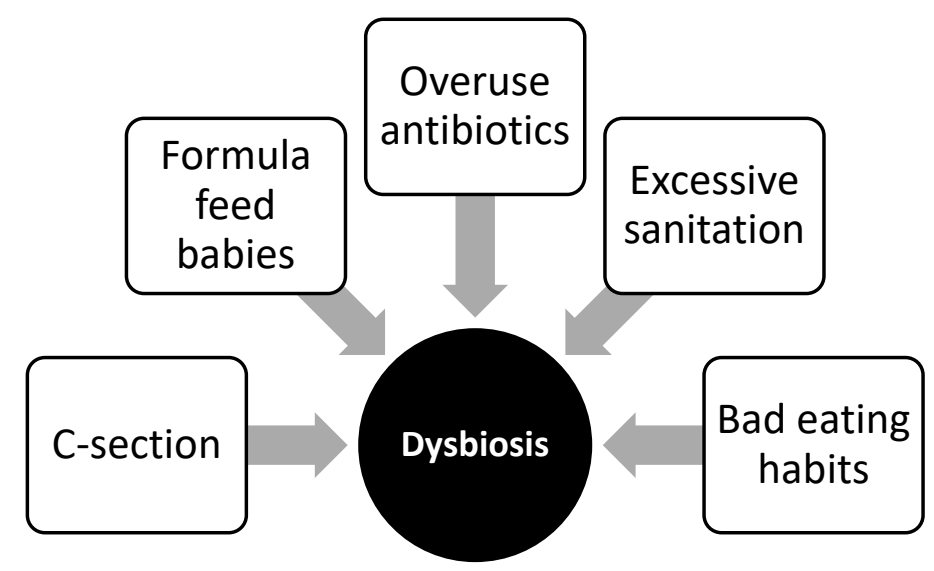

Figure 2: Modern life factors that are causing dysbiosis in the gut microbiota, with consequences to health. It is known that all the points cited have a detrimental consequence on the composition of the gut microbiota. And this starts very early in life, for instance by the way of delivery (C-section) and the excessive use of antibiotics, the use of infant formula to feed babies instead of breast feeding, excessive sanitation, and bad eating habits with lack of fibre consumption as one of the main consequences (17). These early impacts on the gut microbiota may lead to disease and disorder later in life, e.g. obesity (18).

Even though more studies are necessary, the results shown here are promising with regard to the use of a natural food-product that commonly are discarded, to tackle overweight and obesity. 


\section{References}

1. Parfitt J, Barthel M, Macnaughton S. Food waste within food supply chains: quantification and potential for change to 2050. Philos Trans R Soc London B Biol Sci. 2010;365(1554):3065-81.

2. Helkar P, Sahoo A, Patil N. Review: food industry by-products used as a functional food ingredients. Int J Waste Resour. 2016;6(3):1-6.

3. Mirabella N, Castellani V, Sala S. Current options for the valorization of food manufacturing waste: a review. J Clean Prod. 2014;65(Supplement C):28-41.

4. Sharma SK, Bansal S, Mangal M, Dixit AK, Gupta RK, Mangal AK. Utilization of food processing by-products as dietary, functional, and novel fiber: a review. Crit Rev Food Sci Nutr. 2016 Jul 26;56(10):1647-61.

5. Spiker ML, Hiza HAB, Siddiqi SM, Neff RA. Wasted food, wasted nutrients: nutrient loss from wasted food in the United States and comparison to gaps in dietary intake. J Acad Nutr Diet. 2017 Jul;117(7):1031-1040.e22.

6. Juul F, Hemmingsson E. Trends in consumption of ultra-processed foods and obesity in Sweden between 1960 and 2010. Public Health Nutr. 2015 Dec;18(17):3096-107.

7. Louzada ML da C, Baraldi LG, Steele EM, Martins APB, Canella DS, Moubarac J-C, et al. Consumption of ultra-processed foods and obesity in Brazilian adolescents and adults. Prev Med (Baltim). 2015 Dec;81:9-15.

8. FAO, IFAD, UNICEF, WFP, WHO. The state of food security and nutrition in the world 2017. Building resilience for peace and food security. Rome; 2017.

9. Martins ZE, Pinho 0, Ferreira I. Food industry by-products used as functional ingredients of bakery products. Trends Food Sci Technol. 2017;67:106-28.

10. Berry EM, Dernini S, Burlingame B, Meybeck A, Conforti P. Food security and sustainability: can one exist without the other? Public Health Nutr. 2015 Sep;18(13):2293-302.

11. Meybeck A, Gitz V. Sustainable diets within sustainable food systems. Proc Nutr Soc. 2017 Feb;76(1):1-11.

12. Burlingame B, Dernini S. Sustainable Diets and Biodiversity: Directions and Solutions for Policy, Research and Action. In: Sustainable Diets and Biodiversity: Directions and Solutions for Policy, Research and Action International Scientific Symposium, Biodiversity and Sustainable Diets United Against Hunger, FAO Headquarters, Rome, Italy, 3-5 November 2010. Food and Agriculture Organization of the United Nations (FAO); 2012.

13. Meldrum DR, Morris MA, Gambone JC. Obesity pandemic: causes, consequences, and solutions-but do we have the will? Fertil Steril. 2017 Apr;107(4):833-9.

14. WHO. Obesity and Overweight - Fact sheet n. 311. 2017.

15. Cox LM, Yamanishi S, Sohn J, Alekseyenko A V., Leung JM, Cho I, et al. Altering the intestinal microbiota during a critical developmental window 
has lasting metabolic consequences. Cell. 2014 Aug 14;158(4):705-21.

16. Ley RE, Turnbaugh PJ, Klein S, Gordon JI. Microbial ecology: human gut microbes associated with obesity. Nature. 2006 Dec;444(7122):1022-3.

17. Blaser MJ. The theory of disappearing microbiota and the epidemics of chronic diseases. Nat Rev Immunol. 2017 Jul;17(8):461-3.

18. Stanislawski MA, Dabelea D, Wagner BD, Iszatt N, Dahl C, Sontag MK, et al. Gut microbiota in the first 2 years of life and the association with body mass index at age 12 in a Norwegian birth cohort. MBio. 2018 Oct;9(5). 


\section{Acknowledgments}


During my PhD journey I had the luck to meet many people, that definitively contributed to me being here today, writing this last chapter of my thesis. For those, I would like to express my thankfulness.

First of all, Koen: words are not enough to express my gratitude. This $\mathrm{PhD}$ was a "turbulent" one, and no matter what, you were always by my side. Thank you for your support and guidance during these years, which contributed infinitively to my professional development. But especially for always being so comprehensive, for your kindness and optimistic way of looking to the challenges that appeared. I was always amazed by the fact that you always had a solution for everything. Thank you for "taking me with you" to Maastricht University. Without you I would never have the chance to conclude this PhD.

I also would like to thank Prof. Dr. Lubbert Dijkhuizen, Prof. Dr. Susana Saad and Prof. Dr. Daisy Jonkers for accepting being my (co-)promoters, and for your important contributions to the improvement of many manuscripts/thesis.

Thanks to the committee: Prof. Paul Savelkoul, Prof. Ellen Blaak, Prof. Sonia Sayago-Ayerdi and Prof. Kristin Verbeke, for taking time to evaluate my PhD thesis.

Thanks also to Prof. Dr. Fred Brouns and Dr. Freddy Troost. Thanks for the opportunity to study a master in the NL and all support during this period. Doing a master at Maastricht University was essential to introduce me to the "academic world", and the internship at TNO led to my PhD project.

Further, I would like to express my gratitude to Prof. Dr. Henk Schols and Dr. Melliana Jonathan, from Wageningen University. Thank you for opening the doors of the Food Chemistry department, and for your dedication during the time that I spent at WUR. Henk, thank you for providing all necessary tools to pursue my research, and for treating me like as I was a student from you. Melliana, thank you for teaching and guiding me in such kind and patient way. I have learned very much from you. And thanks to all people from the Food Chemistry department which helped me during this period.

I am also thankful to the former TIM team from TNO Zeist, which I had the chance to work with during my master and at the beginning of my PhD: Marjorie, Annet, Rob H., Mark, Wendy, Tom, Jan. Thank you for your support and for sharing your knowledge. 
I would like to give my special thanks to the new TIM team at Maastricht University - Venlo. Rob vD, Cheng, Sanne and Jessica: you guys made my days happier. Thanks to you, I always drove my way to Venlo with a smile on my face. The atmosphere in the labs was delightful, and working with the smelly TIM-2 was always a pleasure with you guys around. It was great when we were all together in one room. I already miss our lunches together, walks, hunting beautiful mushrooms, and the easy talks. You will be always in my heart.

And thanks for all guests that spend some time there, and made my days lighter and brighter. Diogo, Maria I and Maria II, Alessia, Angela, Carol, Lucia.

Dear Prof. Dr. Sonia, I am immensely grateful for your generosity in opening the doors of your home in Venlo to me. I had such good time with you and I hope that we can meet again in the future. Muchas gracias mi querida! Usted sabe que mi casa es su casa!

Dina and Ilse, it was a great pleasure to be your roommate at TNO! Thank you guys for your company and for showing me the best side of the Dutch culture! I will always keep in my heart all good moments that we spent together: our super cool Christmas trip to Germany, lunches, dinner at Dina's place (Dina you are a very talented cook!), fondue with liquid nitrogen, drink's night, barbecue at the park.... wish you all the best for your future! And you are always welcome to come to Brazil and enjoy some caipirinha with me!! Dank je wel meisjes!

To my friends that showed me that distance could not affect our friendship: Lu, Ana Paula, Cyntia, Anne and Begum. No matter for how long we stay far away, I know that our friendship is forever ! Thanks for being part of my life!

Marisol, your friendship is a bless and to meet you was one of the best things that happened to me in the NL. I have learned very much with you since the master, and your strength to get what you desire inspires me. Thank you for being supportive and helping me during the tough moments. I wish you a life filled with love, happiness and success.

Thanks for my husband's family - Wilson, Branca, Cesar, Fernanda and João Pedro, that although far away, always supported me. 
And a warm and especial thank you to my family. To my parents (papi e mami Geraldo e Zélia) for all unconditional love, support and example of perseverance and strength. Vocês são o meu maior exemplo de superação, honestidade e força. Vocês são o meu porto seguro e eu me sinto abençoada por fazer parte da família linda que juntos vocês construíram. Obrigada por me ensinarem a sonhar e a enfrentar os meus medos. Gí e Hérnan, Júnior e Flávia, Matheus e Pedro, obrigada pelo apoio, pela amizade, pelo amor e respeito. Eu admiro muito vocês e desejo que sejamos sempre uma família unida pelo amor. Amo vocês!

I would like to say a very especial thank you for my beloved husband Thiago. Your intelligence inspires me, and each day that I spend with you I admire you more and more. You contributed immensely to me to become a better person. Thank you for your love, support, encouragement and for deciphering $\mathrm{R}$ with me. Muitos dos nosso sonhos já se tornaram realidade, e hoje fazem parte das minhas melhores lembranças. Eu desejo que a nossa união se fortaleça a cada dia, e que a gente nunca perca a capacidade de sonhar. E que juntos a gente continue a trilhar o nosso caminho repleto de amor e felicidade! Te amo!

Thank you!!

Obrigada!!

Carlota 


\section{About the author}




\section{Curriculum vitae}

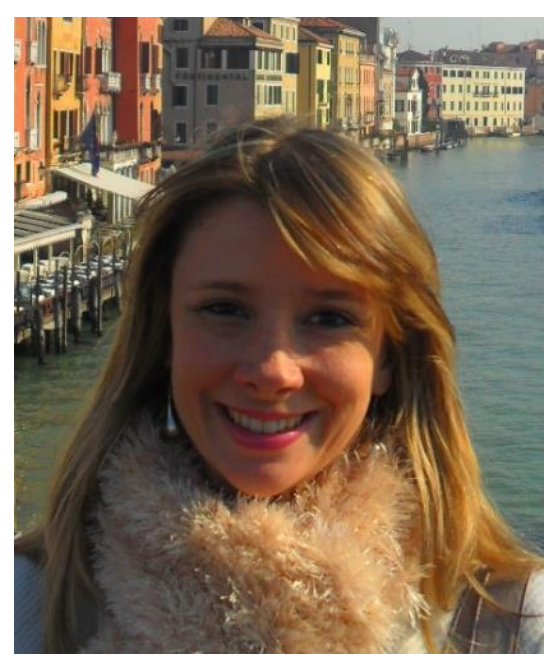

Carlota Bussolo de Souza was born in Florianópolis, Brazil, on the $10^{\text {th }}$ June 1980. She completed her secondary school at "Colégio Energia" in 1999. Afterwards, she studied Nutrition at the Federal University of Santa Catarina (UFSC), Florianópolis, Brazil from 2000 to 2004. During her bachelor studies she became more interested in linking nutrition to gastronomy, which motivated her to pursue a second bachelor - Tourism oriented to Gastronomy. This course was performed at the University of Southern Santa Catarina (UNISUL) from 2003 to 2006. After completing her bachelor in Nutrition, she worked as a dietician in a restaurant in Florianópolis for 1 year. In 2006, when she finished her bachelor in TourismGastronomy, she was selected to participate in the "For.TE Project" - Summer School (Training for Tourism, Food and Wine) in Italy, a partnership program between UNISUL and the "Istituto Giuseppe Maffioli". When she returned from Italy, she decided to start her own company and in 2007, together with her sister, a business plan was developed and "Bussolo Atelier Gastronômico" was created. This small food catering business was focused on supplying high quality pastry delicacies to other companies (coffee shops and restaurants) and events. She worked for 2 years on her own company, before moving to the Netherlands in 2009. In 2010, she was awarded with the "UM High Potential Scholarship" to study the master "Health Food Innovation Management", at Maastricht University at campus Venlo. Her master thesis was entitled "Prebiotic effects of cassava bagasse" and was performed in "The Netherlands Organization for Applied Scientific Research" (TNO), supervised by Dr. Koen Venema and Dr. Freddy Troost. In 2013 she was granted with a scholarship from the Brazilian government, under the program "Science without borders", to pursue a PhD in the Netherlands. She performed her PhD at Maastricht University, under the supervision of Prof. Dr. Koen Venema, Prof. Dr. Susana Saad and Prof. Dr. Lubbert Dijkhuizen. The results of her research are described in this thesis. 


\section{List of publications}

Rossi, C. E., Bussolo C., Proença, R.P.C. ISO 14000 no Processo Produtivo de Refeições: Implantação e Avaliação de um Sistema de Gestão Ambiental. Nutrição em Pauta, v. 101, p. 21-25, 2010. - In Portuguese

Bussolo de Souza C., Roeselers G., Troost F., Jonkers D., Koenen M.E., Venema K. Prebiotic effects of cassava bagasse in TNO's in vitro model of the colon in lean versus obese microbiota. Journal of Functional Foods. 2014;11(C):210-20.

Aguirre M., De Souza C.B., Venema K. The gut microbiota from lean and obese subjects contribute differently to the fermentation of arabinogalactan and inulin. PLoS One. 2016 Jul 1;11(7).

Bussolo de Souza C., Venema K. Microbiota intestinal em indivíduos eutróficos e obesos. In: Faintuch J, editor. Microbioma, disbiose, probióticos e bacteroterapia. 1st ed. São Paulo: Manole; 2017 (pp. 164-177). - In Portuguese

de Souza CB, Jonathan M, Saad SMI, Schols HA, Venema K. Characterization and in vitro digestibility of by-products from Brazilian food industry: cassava bagasse, orange bagasse and passion fruit peel. Bioact Carbohydrates Diet Fibre. 2018; (16):90-99.

Larsen N., Bussolo de Souza C., Cahú T. B., Krych L., Wiese M., Kot W., Hansen K. M., Blennow A., Venema K., Jespersen L. Potential of pectins to beneficially modulate the gut microbiota depends on their structural properties. Submitted

Bussolo de Souza C, Jonathan M., Saad S.M.I., Schols H.A., Venema K. Degradation of fibres from fruit by-products allows selective modulation of gut bacteria in an in vitro model of the proximal colon. Submitted

Bussolo de Souza, C., Saad, S. M. I., Venema, K. Lean and obese microbiota: differences in in vitro fermentation of by-products from Brazilian food industry. In preparation 